\title{
Cosmic Evolution Physics (II) for Inflationary Universe with Rip-Rebound Clusters
}

\author{
Li Zongcheng \\ ( Research Lab of Interdisciplinary Science, Soochow University, Soochow, P. R. CHINA )
}

\begin{abstract}
Under the big unified framework of quantum gravitational field and quantum repulsive field, the earlier in this series of work has comprehensively expanded the general relativity and loop quantum ring gravitation theory, set up the quantum repulsive field equation corresponding to the quantum gravitational field equation, and then established the quantum hedge-unified field equation describing the interaction of quantum gravitational field and quantum repulsive field; On this basis, the quantum repulsive universe equation corresponding to the quantum gravitational universe equation has be established, thus a set of new equations describing the expansive universe model under the condition of fully considering the hedge effect of quantum gravitational field and quantum repulsion field, which can be called the expansive universe equation of quantum hedge-unified field theory, has be established. Now, let's go deep into the arche-unified physics behind the creation of the universe. Although all kinds of inflationary universe models can solve cosmological problems to a certain extent, there is no natural and reasonable analysis and explanation for all cosmological and physical consequences. For example, it is impossible to predict a large tensor-scale ratio and a relatively large spectral index run at the same time. At present, string theory, membrane theory, loop quantum gravity theory, super-gravitational holography principle and $\mathrm{M}$ theory, which are the theoretical basis of cosmology, are basically established by simplification in the case of breaking away from the extreme background conditions of the universe. While cosmology, which is deficient in nature, cannot provide strong support for particle physics to further advance to a wider field and a deeper level. Due to the lack of new ideas, the construction of existing theoretical models is difficult. Therefore, the existing research can only be further promoted by new astronomical observation. Unlike existing studies, we concentrate on the emergence of the big rip-rebound clusters of quantum chaotic-reticulate distribution between the outwards-push of the universe as a whole (related to pseudo-vacuum energy, Higgs field, dark energy, etc.) and the inwards-pull of the universe in local scale (related to dark matter, quantum gravity and initial gravity separated from high-dimensional supergravity, and the initial strong force separated from the grand unified force subsequently). On the one hand, we find a great impetus to make the inflation of the universe into firstly-imposed suppression, then-imposed acceleration and finally-imposed deceleration, which really explains why the universe expands at a critical rate and has the same temperature in different regions, on the other hand, we find a fundamental unit of matter and its emerging clusters evolving in the big bang, inflation and expansion of the universe accompanied by the great rip-rebound clusters of quantum chaos-reticulate distribution, which can be reduced to an arche-pulsator of neither punctiform particle nor linear string. From these two aspects, it can been seen that the evolution of the early universe is much more complicated than people originally thought. The complexity of the super inflation of the universe is mainly reflected in the big rip-rebound cluster emerging behind the gravitational disturbance (the pressure and tensor disturbance related to dark matter), the repulsive disturbance (the pressure and scalar disturbance related to dark energy), the cosmological disturbance (related to the interaction between high-dimensional supergravity and high-dimensional superrepulsion) This is a system of nonlinear stochastic differential dynamics, or a quantum statistical physical process of nonlinear
\end{abstract}


nonequilibrium state. In the new research paradigms established in this series, the arche-conjugation between the high-dimensional supergravity and the high-dimensional superrepulsion, as well as between the quantum gravity and the quantum repulsion, is higher than the supersymmetry, and then becomes the core concept of the new theory. In this series, cluster-inflating configuration, spin network space and cosmic paradigm vectors are proposed, so the model of the super-inflated universe with quantum chaos-reticulate distribution emerged under the great impetus. The new model reveals that the unified inflation of the universe as a whole and the local inflation of the universe emerge together in the great impetus of the big rip-rebound cluster of quantum chaos-reticulate distribution. As a spin network model variable, the spatiotemporal mode variable is a vector composed of a series of variables, which variables include: dimension, curvature, range, expansion speed, dynamics, quantization, chaotic-reticulate distribution (inhomogeneity, concentration, fluctuation), synergy, and so on. Between particle physics and cosmology, this series of papers proposes the cosmic quantum evolution mode, the physical-sphere distributing configuration and the interaction situation variables, organizes the concepts, variables and models of these three levels, and establishes a systematic super-synergy paradigm. In this paradigm, the general Langevan equation and the general Fokker-Planck equation are established. For the quantum cosmic-sphere, a series of trend parameters are introduced to establish the dynamics of quantum gravitational mode and its master equation, and then to establish the operator distribution function and its motion equation of the action mode. For the Tachyon of upheaval in the rip-rebound-inflating of the universe, the hypothesis on the bifurcate-chaos wave of quantum is given, the quantum theory of the early photons in upheaval is set up, and the analysis on the particles of the ultrahigh energy cosmic ray from the $\gamma$ ray burst is made. Finally, using Wigner-Ville distribution as a nonlinear time-frequency distribution, we establish the ultra-synergistic field equation of quantum-classic unifying action-sphere.

Keyword: supergravity; superrepulsion; rip-rebound cluster; super-inflationary universe; ultra-synergy dynamical equation

PACS: 04.25.dg; 04.60.-m; 11.25.Yb; 12.10.-g; 98.80.Cq; 98.80.Qc

\section{Introduction}

Historically, thinking about the inflation of the universe has been based on a number of different perspectives. From the old inflationary universe model (A. Guth) ${ }^{[1]}$ to the new inflationary universe model (A. Linde ${ }^{[2]}$, from the chaotic inflationary universe model (A. Linde) ${ }^{[3]}$ to the extended inflationary universe model ${ }^{[4][5]}$, from the multi-field inflationary universe model ${ }^{[6][7]}$ to the hybrid inflationary universe model (A. Linde) ${ }^{[8][9]}$, from the natural inflationary universe model ${ }^{[10]}$ to the inflation universe model with non-regular scalar field ${ }^{[11]}$, from the K-inflationary universe model ${ }^{[12]-[14]}$ to the inflationary universe model with non-regular kinetic energy ${ }^{[15]-[17]}$, there were various inflationary universe models later, for example, tachyon model ${ }^{[18]-[20]}$, Dirac-Born-Infeld model $(\mathrm{DBI})^{[21]}$, multi tachyon inflation model ${ }^{[22]-[24]}$, phantom inflation model ${ }^{[25]-[29]}$, non-commutative spatiotemporal inflation model ${ }^{[30]-[34]}$, double inflation model, rebound inflation model, self-anisotropic inflation model, deformed phase space inflation model, strange inflation model, viscous fluid inflation model, quintessential inflation model, and so on ${ }^{[35]-[38]}$, there appeared Hadley-Hawking initial state, string theory cosmology, loop quantum cosmology, transmode inflation, string gas cosmology, fire disaster cosmology, and so on ${ }^{[39]-[44]}$.

However, there is a problem in the existing inflation models: they can not predict a large tensorscale ratio and a relatively large spectral index run at the same time. In order to be consistent with the 
observations, some studies have considered the model outside the single scalar field, the simplest of which is to consider the role of cosmological constant in the period of the inflation. The cosmological constant is usually ignored because it alone can only predict a scale invariant power spectrum, that is, the Harrizon-Zel'dovish spectrum $n_{s}=1$, which has been excluded from the Planck experiment at a confidence level of more than $5 \sigma$. See reference [32]. However, some researchers have found that when the universe is dominated by the inflation field and cosmological constant at the same time, the situation changes. It can not only predict a red spectrum $n_{s}<1$, but also predict a large scale ratio. At the same time, the e-folding number is large enough to meet the needs of solving the flatness problem.

For the inflationary universe, once a large spectral index run is confirmed, a mechanism is needed to explain it. People don't understand why the prediction of the inflation is highly sensitive to the initial conditions, or why the inflation will lead to the eternal inflation, which will lead to multiple universes — there are infinite theoretical output results ${ }^{[45]-[47]}$. Logically, if the output of the inflation is highly dependent on the initial conditions that have not been well understood, then the result cannot be determined. Moreover, if the inflation produces multiple universes, as A. Guth said, "anything that can happen will happen", then it will make any discussion of the prophecy meaningless ${ }^{[48]-[50]}$. Different from the standard model of particle physics, even if all parameters are fixed, the inflation model will still give infinite results, and no one result is more special than the other, which makes the inflation immune to any experimental test.

We know that the Higgs boson discovered by Peter W. Higgs and Francois Englert, et al, is one of the core parts of the standard model of particle physics, which describes how the world we live in is made $u^{[51]-[53]}$. The completion of the standard model requires the existence of a particle, that is the Higgs particle, which originates from an invisible field that fills the whole space. It is through the interaction with this field that a particle gains mass. Engler's and Higgs' theories describe this process. In 2012, CERN particle physics experiments confirmed the existence of Higgs particles.

The universe may be symmetrical at its birth, but after only $10^{-11}$ seconds of the Big Bang, the Higgs particle deviates from the center of symmetry and reaches its lowest energy state, thus breaking the symmetry ${ }^{[54]-[56]}$. There is not enough observational evidence to explain why there are more baryons than antibaryons in the universe. To explain this ratio, the Sakharov situation must occur sometime after inflation. When such a scenario is considered, although this phenomenon is observed in the experiments of particle physics, the observed asymmetry is too small to satisfy the observed asymmetry in the universe. Although the discovery of Higgs particles eventually fills in the last missing link in the standard model, the standard model itself is not the last link to uncover the ultimate mystery of the universe. Andrei Linde recognizes in the advance of the next step that there is no need to make any special provision for the Planck-sized space-time zone that expands into our universe ${ }^{[5]-[7]}$. If that's a part of some larger space-time region where all scalar fields are valid, then only those space-time regions where scalar fields can trigger a inflation can lead to a universe like ours. Lind calls this "chaotic inflation", because scalar fields can take any number at different locations in the early super-universe; this is the standard version of today's inflation theory, which can be regarded as an example of theoretical thinking related to the principle of anthropology (note that the use of the word "chaos" here is similar to the meaning of the daily mess. It has nothing to do with the number of subjects called chaos theory.

The idea of chaotic inflation has led to the significant progress in inflation theory so far ${ }^{[57]-[59]}$. The 
main question that standard Big Bang cosmology fails to answer is what is "before" the singularity. It is often said that this problem is meaningless, because time begins at the singularity. However, the chaotic inflation theory holds that our universe is formed by quantum fluctuations in a pre-existing spatiotemporal region, and that it can also create an inflationary zone through a completely equivalent process in our own universe ${ }^{[60]-[62]}$. In short, the new universe can be separated from our universe, our universe itself may be separated from another universe, which is a process without beginning or ending. One way of saying about this topic is that the "separation" process takes place through black holes. Whenever a black hole collapses into a singularity, it "jumps" out and enters another set of space-time dimensions, creating a new booming universe, which is called the baby universe ${ }^{[63]-[65]}$. The two Dicke coincidences are manifested in cosmic flatness and cosmological constants. In the existing cognition, the macrogeometry of the universe is basically divided into three categories: hyperbolic geometry (open universe), spherical geometry (closed universe) and Euclidean geometry (flat universe). Observed data from the microwave background of the universe verify that the universe is flat, and the error is less than $10 \%$, which makes the flatness problem more significant. Relevant researchers are always considering the reasons why the universe becomes flat ${ }^{[66]-[68]}$.

For the real solution of the problem, existing models of inflation, dark energy and dark matter need further improvement and development, rather than a tactful avoidance. The Higgs field can not be used to fully explain many problems of the early universe ${ }^{[45]-[47]}$. In addition, Max Tegmark believes that ${ }^{[6]-[8]}$ the parallel universe is not a theory, but a theoretical prediction. Various models of the inflation universe depend on the choice of parameters, including the dependence on the shape and initial conditions of the energy density curve of inflation. In our view, the Higgs mechanism is only one aspect of a much larger and more complex ultra-synergy mechanism than previously thought.

Up to now, there does not seem to be a clear answer that is in line with human wishes, as people expect. The theory and model of the Big Bang are not impeccable and without loopholes, nor can people prove that it fully conforms to the real situation of the evolution of the universe. Therefore, today's cosmology is far from being able to predict the ultimate outcome of the universe. It is still unknown whether string theory can successfully explain the universe which are based on all the forces and substances known in the physical world, and its extreme cases in which quantum mechanics and general relativity is needed to be applied to "black holes" and "Big Bang of the Universe", etc. How to use string theory to explain the initial starting point of the Big Bang is still an unsolved problem.

In the situation of neither improving both quantum mechanics nor relativistic physics, some new theories and models have been developed to explore the combination of quantum mechanics and general relativity, such as superstring theory, superfilm theory, loop quantum gravity theory ${ }^{[50][66][69]}$, holographic principle, M theory (membrane theory or mystery theory) and reversed Sitter space (AdS) / conformal field theory (CFT). In the attempt to improve quantum mechanics, there are only a few theories and models to explore the combination of quantum mechanics and general relativity, such as Roger Penrose's theory of spatiotemporal torsion and Alain Connes' new exploration without relativity and quantum mechanics ${ }^{[70]-[72]}$.

Although all kinds of inflationary universe models can solve cosmological problems to a certain extent, there is no natural and reasonable analysis and explanation for all cosmological and physical consequences. In the scientific exploration of approaching the arche (origin) of all things, physics is closely related to geometry, so that there is neither pure physics (such as Euclidean quantum gravitation, 
torsion) nor pure geometry (such as non exchangeable geometry, acoustic gauge), only physical geometry and geometric physics. In the exploration of approaching the arche (origin) of all things, physics is closely related to ontology, so that there is neither pure physics nor pure ontology here, only physical ontology and ontological physics. Finally, we should and must return to the noumenon of natural science.

At present, string theory, membrane theory, loop quantum gravity theory, super-gravitational holography principle and $\mathrm{M}$ theory, which are the theoretical basis of cosmology, are basically established by simplification in the case of breaking away from the extreme background conditions of the universe. While cosmology, which is deficient in nature, cannot provide strong support for particle physics to further advance to a wider field and a deeper level. Due to the lack of new ideas, the construction of existing theoretical models is difficult. Therefore, the existing research can only be further promoted by new astronomical observation. For the universe in the formation of fundamental particles and the fundamental particles in the evolution of the universe, whether the reduction under the principle of simplicity or the synthesis under the principle of complexity, it is not enough to establish a reasonable theoretical basis. The best way of research should and can only be to form a summary of relevant research on particle physics and cosmology under the principle of fusion.

Therefore, it is necessary to do more in-depth systematic research on the interaction between the expansion force caused by Higgs field and all the internal gravitation, as well as the interaction between the expansion force caused by Higgs field and all the internal strong and electro-weak forces.

Let's further expand our thinking space:

Before the creation of our universe, the whole world system of multi-verse was filled with infinite pseudo-vacuum oceans, where high-density energies converged. Every fluctuation of the initial vacuum implies the collective birth and death of particles.

The pseudo-vacuum, which accumulates extremely high density energy, forms negative pressure on the universe and then converts it into repulsive force ${ }^{[54][60][62]}$, opening up a new space-time for the universe. With the rapid expansion of space and time, the Higgs field began to over-cool, and the pseudo-vacuum began to increase, causing a larger scale of expansion, leading to the enlarging of space and time. Through the non-linear feedback, the pseudo-vacuum increases continuously, and the initial gravitation and strong force separated from the high dimensional supergravity form a big rip-rebound cluster of quantum chaotic-reticulate distribution at macro and micro levels respectively with the expansion force caused by Higgs field, thus forming a great impetus of first suppression, later acceleration and last deceleration of the universe, and then causing the universe to enlarge exponentially.

Under the extreme conditions of the initial universe, in the a big rip-rebound cluster of quantum chaotic-reticulate distribution emerging between the initial gravity separated from quantum gravity and the expansive force caused by Higgs field, and then between the strong force separated from the grand unified gauge field and the expansive force caused by Higgs field, the basic matter unit successively becomes the arche-chaotic pulsator, quasi-spherical pulsator and spherical pulsator. Various wave modes of the arche-pulsator form various particles.

In the initial stage of the creation of the universe, the interaction between the expansive force caused by the Higgs field and all the internal gravitational forces, as well as the interaction between the expansive force caused by the Higgs field and all the internal strong forces, become a big impetus for 
the acceleration or deceleration of the cosmic inflation, thus becoming an extremely important factor that can not be ignored. The higher the density of cosmic energy, the higher the degree of synergy of the interaction system. This ultra-synergy between particles and the universe creates a sudden force of cosmic inflation, which leads to the exponential increase of cosmic inflation. The faster the universe cools, the stronger the power of space-time.

Now, let's go deep into the arche-unified physics behind the creation of the universe.

In the new research paradigms established in this series, the arche-conjugation between the high-dimensional supergravity and the high-dimensional superrepulsion, as well as between the quantum gravity and the quantum repulsion, is higher than the supersymmetry, and then becomes the core concept of the new theory.

Under the big unified framework of quantum gravitational field and quantum repulsive field, the earlier in this series of work has comprehensively expanded the general relativity and loop quantum ring gravitation theory, set up the quantum repulsive field equation corresponding to the quantum gravitational field equation, and then established the quantum hedge-unified field equation describing the interaction of quantum gravitational field and quantum repulsive field; On this basis, the quantum repulsive universe equation corresponding to the quantum gravitational universe equation has be established, thus a set of new equations describing the expansive universe model under the condition of fully considering the hedge effect of quantum gravitational field and quantum repulsion field, which can be called the expansive universe equation of quantum hedge-unified field theory, has be set up.

In order to reduce the excessive dependence of various inflationary universe models on the initial conditions ${ }^{[49]-[51]}$, we will fully consider and study a great impetus, which is a super-synergistic dynamic system between particles and the universe. Through this great impetus, we can reduce the excessive dependence of the inflationary universe model on the initial conditions to a large extent, and increase the reasonable dependence of the inflationary universe model on the evolution process to a large extent.

Unlike existing studies, we concentrate on the emergence of the big rip-rebound clusters of quantum chaotic-reticulate distribution between the outwards-push of the universe as a whole (related to pseudo- vacuum energy, Higgs field, dark energy, etc.) and the inwards-pull of the universe in local scale (related to dark matter, quantum gravity and initial gravity separated from high-dimensional supergravity, and the initial strong force separated from the grand unified force subsequently). On the one hand, we find a great impetus to make the inflation of the universe into firstly-imposed suppression, then-imposed acceleration and finally-imposed deceleration, which really explains why the universe expands at a critical rate and has the same temperature in different regions, on the other hand, we find a fundamental unit of matter and its emerging clusters evolving in the big bang, inflation and expansion of the universe accompanied by the great rip-rebound clusters of quantum chaos-reticulate distribution, which can be reduced to an arche-pulsator of neither punctiform particle nor line string. From these two aspects, it can been seen that the evolution of the early universe is much more complicated than people originally thought.

We further find that at the initial moment for fundamental forces to separate, both the quantum gravi-source and the gravi-source, as well as the strong force source and the electro-weak force source, are very powerful, and between ever-increasing interaction sources there should form superimposed amplification effect. These ultra-synergy in several phase transitions during the creation of the universe 
are the big impetus for the rip-rebound- inflation and further expansion of the universe. With the big bang, inflation and expansion of the universe, the quantum phy-sphere approaching Planck energy scale has experienced several phase transitions. Quantum gravitation, gravitation and gauge field force have been separated from the high-dimensional supergravity successively, and the big rip-rebound cluster with the network distribution of quantum chaos emerges between the universe and particles, thus forming the super synergy.

On the basis of the previous discussion in this series, the quantum phy-sphere is proposed and studied in this paper, which is a system of all things accumulation centered on quantum gravitational sources. It is not only limited to the scope of quantum systems, but also involves the scope of macro-systems. It is not only limited to material or physical things, but also involves the complexity with a variety of elements. Its range of existence and evolution is generally slightly larger than the event horizon. In the quantum phy-sphere, the quantum gravitational source is not unique, but may be multiple.

The basic meaning of the super-inflating universe model is that our universe in four-dimensional space-time is an expansive bulk emerging through continuous breakthrouth to the compactness of four-dimensional space-time in the Big Bang and the inflation from a high-dimensional space (KaruzaKlein theory). In the case that all the extra dimensions outside the four dimensions are compacted, although the universe contains other extra dimensions, there is no reference frame for these extra dimensions in this "expansive bulk", and other four-dimensional space-time "bulks" can also pass through this high-dimensional space-time "bulk" and interact with this high-dimensional time-space "bulks", so as to produce some effects that are not visible in standard cosmological models.

For the cosmic ecosphere of the multi-verse world, this series sets up a larger theoretical framework at a deeper level, which combines the creation of the universe with the unification of basic interactions. On the one hand, we re-study the primitive unification of fundamental interactions under the extreme conditions of the creation of the universe; on the other hand, we re-study the creation of the universe on the basis of the primitive unification of fundamental interactions. Therefore, this series of work provides a more perfect and reasonable description, analysis and interpretation as far as possible, and brings a more approach to the world noumenon for particle physics, astrophysics and cosmology.

The complexity of the super inflation of the universe is mainly reflected in the big rip-rebound cluster emerging behind the gravitational disturbance (the pressure and tensor disturbance related to dark matter), the repulsive disturbance (the pressure and scalar disturbance related to dark energy), the cosmological disturbance (related to the interaction between high-dimensional supergravity and high-dimensional superrepulsion). This is a system of nonlinear stochastic differential dynamics, or a quantum statistical physical process of nonlinear nonequilibrium state.

In the new research paradigms established in this series, the arche-conjugation between the high-dimensional supergravity and the high-dimensional superrepulsion, as well as between the quantum gravity and the quantum repulsion, is higher than the supersymmetry, and then becomes the core concept of the new theory.

In this series, cluster-inflating configuration, spin network space and cosmic paradigm vectors are proposed, so the model of the super-inflated universe with quantum chaos-reticulate distribution emerged under the great impetus. The new model reveals that the unified inflation of the universe as a whole and the local inflation of the universe emerge together in the great impetus of the big rip-rebound 
cluster of quantum chaos-reticulate distribution. As a spin network model variable, the spatiotemporal mode variable is a vector composed of a series of variables, which variables include: dimension, curvature, range, expansive speed, dynamics, quantizing, chaos-reticulated distribution (inhomogeneity, concentration, fluctuation), synergy, and so on. Between particle physics and cosmology, this series of papers proposes the cosmic quantum evolution mode, the physical-sphere distributing configuration and the interaction situation variables, organizes the concepts, variables and models of these three levels, and establishes a systematic super-synergy paradigm. In this paradigm, the general Langevan equation and the general Fokker-Planck equation are established. For the quantum cosmic-sphere, a series of trend parameters are introduced to establish the dynamics of quantum gravitational mode and its master equation, and then to establish the operator distribution function and its motion equation of the action mode. For the Tachyon of upheaval in the rip-rebound-inflating of the universe, the hypothesis on the bifurcate-chaos wave of quantum is given, the quantum theory of the early photons in upheaval is set up, and the analysis on the particles of the ultrahigh energy cosmic ray from the $\gamma$ ray burst is made. Finally, using Wigner-Ville distribution as a nonlinear time-frequency distribution, we establish the ultra-synergistic field equation of quantum-classic unifying action-sphere.

\section{Research Framework between Particles and the Universe}

As an important step in the establishment of ultra-synergy physics between particle physics and cosmology, we now combine the study of cosmic synergistic-evolution mode (cosmic quantum spacetime and cosmic quantum field) with the study of the phy-sphere organization configuration and the study of physical action situation, to put up the unified research framework for the evolution of nature.

Facing the cosmic ecosphere in the multi-world, the central content of the unified research framework for the evolution of nature is summarized as the research on the great impetus mechanism of cosmic evolution. This research includes three levels of mechanism research, namely, the research on the great impetus mechanism of the cosmic Big Bang, the research on the great impetus mechanism of cosmic inflation and the research on the great impetus mechanism of cosmic expansion.

Figure 1 (Li Zongcheng, 2019) gives a framework for ultra-synergy physics between particle physics and cosmology. If we rewrite the formula ( $1 b)$ in Friedmann equation

$$
\begin{gathered}
H^{2}=\frac{\dot{a}^{2}}{a^{2}}=\frac{8 \pi G}{3} \rho-\frac{k}{a^{2}}+\frac{\Lambda}{3} \\
\frac{\ddot{a}}{a}=-\frac{4 \pi G}{3}(\rho+3 p)+\frac{\Lambda}{3}
\end{gathered}
$$

as follows:

$$
F=-\frac{G M}{R^{2}}+\frac{\Lambda}{3} R, \quad R=a
$$

it can be seen that the repulsion force produced by cosmological constant increases with the increase of distance. Whether the expansion of the universe slows down or accelerates depends on the contrast between the two terms on the right of equation ( 2 ), that is, the counterbalance between the gravitation 
of matter and the repulsion of cosmological constant. In the distant past, cold dark matter dominated the universe, slowing down the expansion of the universe; about 5 billion years ago, cosmological constants began to dominate gradually, and the expansion of the universe began to accelerate.

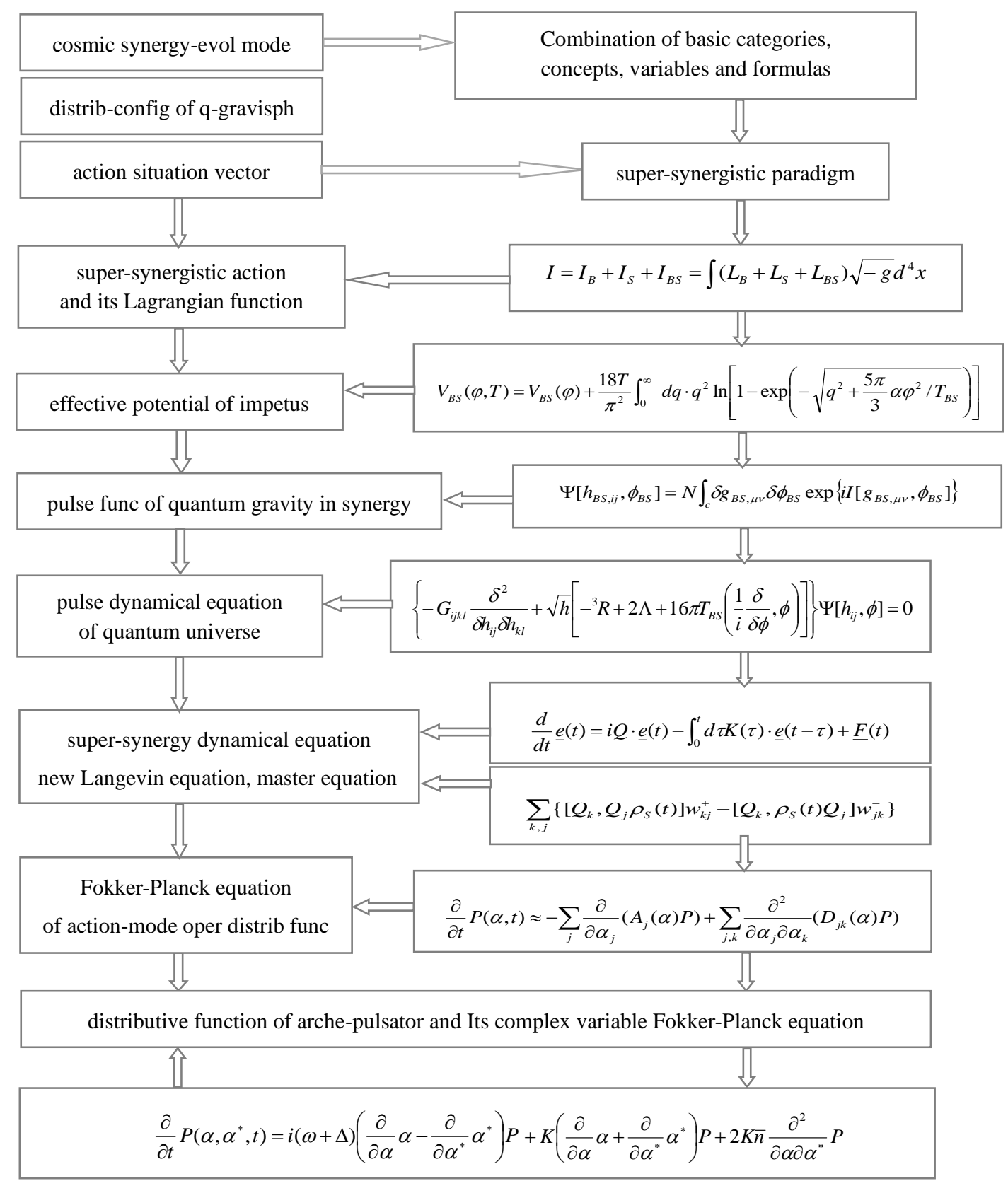

Fig. 1 Research Framework of Ultra-Synergy Physics between Particle Physics and Cosmology

The basic contents of the research on the great impetus mechanism of the cosmic Big Bang (10 ${ }^{19}$ $\left.\mathrm{GeV}, 10^{-44} \mathrm{sec} \sim 10^{-36} \mathrm{sec}\right)$ :

In the big rip-rebound cluster with quantum chaotic network distribution between the outwards-push from the Big Bang of the universe as a whole (related to the energy accumulation 
mechanism of pseudo-vacuum) and the inwards-pull of the universe in local scale (related to the separation mechanism of quantum gravity), many arche-chaotic pulsators are formed in the quantum chaotic universe. In this regard, we study the ultra-synergy dynamic process between the arche-chaotic pulsator and the quantum chaotic universe.

In the big rip-rebound cluster with quantum chaotic network distribution between the outwards-push from the Big Bang of the universe as a whole (related to the energy release mechanism of pseudo-vacuum) and the inwards-pull of local initial gravitation (related to the separation mechanism of quantum gravity), many chaotic bulks (the group of arche-chaotic pulsators) are formed in the quantum chaotic universe. In this regard, we study the ultra-synergy dynamic process between the group of arche-chaotic pulsators and the quantum chaotic universe.

The contents of the research include:

relevant Initial spatiotemporal mode vectors and their basic dynamic equations

generalized master equation, generalized Langevin equation, operator distribution function, Fokker-Planck equation and average value equation of the related organization configuration of quantum gravisphere

relevant trend parameters and basic vector dynamic equation of interaction situation

The basic contents of the research on the great impetus mechanism of the cosmic inflation ( $2 \times$ $\left.10^{16} \mathrm{GeV}, 10^{-38} \mathrm{sec} \sim 10^{-33} \mathrm{sec}\right)$ :

In the big rip-rebound cluster with quantum chaotic network distribution between the outwards-push from the inflation of the universe as a whole (related to the pseudo-vacuum, Higgs mechanism and the synergy of great impetus) and the inwards-pull of local gravitation (related to the separation mechanism of quantum gravity), the cosmic inflation field should produce many bubbles in exponentially ever-increasing. In this regard, we study the ultra-synergy dynamic process between the group of quasi-spherical pulsators and the whole of the quantum universe.

In the big rip-rebound cluster with quantum chaotic network distribution between the outwards-push from the inflation of the universe as a whole (related to the pseudo-vacuum, Higgs mechanism and the synergy of great impetus) and the inwards-pull of initial strong interaction (related to the separation mechanism of gauge field), a large number of fully chaotic pulsators should evolve into quasi-spherical pulsators. In this regard, we study the ultra-synergy dynamic process between the quasi-spherical pulsators and the quantum universe.

The contents of the research include:

relevant evolutionary mode vectors of the quantum universe and its basic dynamic equations

generalized master equation, generalized Langevin equation, operator distribution function, Fokker-Planck equation and average value equation of the relevant organization configuration of quantum universe

relevant trend parameters and basic vector dynamic equation of interaction situation

The existing theory can confirm that the $T_{\mathrm{c}} \approx 10^{14} \mathrm{GeV}$ is the phase transition temperature from the grand unified phase to $S U(3) \times S U(2) \times U(1)$. The corresponding time can be derived from the following formula:

$$
T^{2}=\sqrt{\frac{45}{\pi^{3} N_{e f f}}} \frac{M_{p}}{4 t} \quad \text { (radiation-dominated period) }
$$


here $N_{\text {eff }}$ is taken as the typical value of the grand unified theory, $N_{\text {eff }} \approx 100$, and we get $t_{c} \approx 10^{-33} \mathrm{sec}$.

In the framework of the great unity of quantum gravitational field and quantum repulsive field, the scalar disturbance is regarded on the one hand as the reflection of repulsive effect, the tensor disturbance is regarded on the other hand as the reflection of gravitational effect. In the initial stage of the big ripping, due to the mutual restraint of quantum repulsion and quantum gravity, the scalar and tensor perturbations are not obvious. However, at the end of the big ripping, the scalar and tensor perturbations are very significant, and there is a large power spectral exponential run.

During the inflation of the universe, the tensor perturbation mode stimulated the $B$-mode of photon polarization, and this signal has been observed by BICEP 2 experimental group ${ }^{[73]-[76]}$. In fact, the tensor disturbance of the gauge field corresponds to the forming of gravitational wave. The observation of $B$-mode means that the gravitational wave is observed, but the gravitational wave is excited in the early universe, which is called the primary gravitational wave. Through the measurement of $B$-mode, the magnitude of tensor disturbance can be estimated, which is usually described by a quantity called tensor-scale ratio. By the data analysis of BICEP 2 experimental group, the tensor-scale ratio was limited to $r=0.20_{-0.05}^{+0.07}$ ( $68 \% \mathrm{CL}$ ), and the possibility of $r=0$ was excluded on the confidence level of 7.0 $\sigma$. With the limit of the tensor-scale ratio and the spectral index, we can distinguish and exclude some inflation models, such as small field inflation model.

However, there are some inconsistencies between the results of BICEP 2 and Planck experimental groups. According to the data of Planck + WP + high L, the spectral index is limited to $n_{s}=0.9600 \pm 0$. 0071, while the tensor-scale ratio can be almost ignored $r_{0.002}<0.11$ ( 95\% CL ) ${ }^{[36][74]}$. However, when using the run of spectral index as an independent parameter to fit the same data, it can be found that $n_{s}=0.95700 \pm 0.0075, r_{0.002}<0.26$ and $n_{s}^{\prime}=-0.022_{-0.021}^{+0.020}(95 \% \mathrm{CL})$. That is to say, in order to make these two results coincide with each other, theoretically there should be a relatively large spectral index run, such as $n_{s}^{\prime} \leq-0.001$. Of course, the size here refers to the size of its absolute value.

On the other hand, the inflationary universe is regarded as the most economical choice to solve the problem of universe flatness in the big bang cosmology ${ }^{[1][2][77]}$. Moreover, the quantum perturbations of the inflation field and the background gauge field will become the seeds in the formation of the large-scale cosmic structure. The discovery of photon polarized $B$-mode also confirms that the universe did inflate in the early days. In the simplest model of the inflationary universe, a scalar field called an inflaton drives the universe to expand faster. The scalar field needs a very flat potential energy $V(\varphi)$ in order to have a long enough inflation time in the early universe. When the inflation starts, it will slowly roll from the place with high potential energy to the place with low potential energy. This process is called slow-roll inflation. For example, in the chaotic inflation model ${ }^{[3]}$, there is $V \sim \varphi^{n}$; in the natural inflation model, there is $V \sim V_{0}(\cos \varphi / f+1)^{[10]}$.

In these models of the inflation, the order of magnitude of spectral index deviation from Harrison Zeldovich scale invariant spectrum is $n_{s}-1 \sim O\left(10^{-2}\right)$, and the order of magnitude of spectral index run is $n^{\prime} \sim O\left(10^{-4}\right)$. It can be seen that for these models, it is not easy to achieve a large tensor-scale ratio and a large spectral index run at the same time. This problem is particularly discussed in reference [78], but there is no solution. In addition, reference[79] also points out that for the slow-rolling inflation, it is difficult to keep the potential energy of the inflation field with a relatively large third derivative 
while keeping the first derivative and the second derivative relatively small, unless the required e-folding number $N$ is relatively small, for example, when $N \approx 20 \sim 30$. The same conclusion is given in reference [80].

The extreme flatness of space and time means that the expansion of the universe and the resistance of gravity are balanced, so that the universe is precisely at the boundary between the eternal expansion and the eventual collapse to big crumbling.

The basic contents of the research on the great impetus mechanism of the early cosmic expansion $\left(10^{-32} \mathrm{sec} \sim 10^{-9} \mathrm{sec}\right)$ :

In the big rip-rebound cluster with quantum chaotic network distribution between the outwardspush from the expansion of the universe as a whole (related to the excessive freezing, Higgs mechanism and the synergy of great impetus) and the inwards-pull of microscopic strong interaction and weak force (related to the separation mechanism of gauge field), many quasi-spherical pulsators should evolve into charged particles, photons and leptons. In this regard, we study the ultra-synergy dynamic process between spherical pulsators and the universe.

In the big rip-rebound cluster with quantum chaotic network distribution between the outwardspush from the expansion of the universe as a whole (related to the excessive freezing, Higgs mechanism and the synergy of great impetus) and the inwards-pull of local gravitation and electromagnetic force (related to the separation mechanism of quantum gravity and gauge field), the cosmic inflation field should produce many bubbles in exponentially ever-increasing. In this regard, we study the ultra-synergy dynamic process between the group of spherical pulsators and the whole of the universe.

The contents of the research include:

relevant evolutionary mode vectors of the universe and its basic dynamic equations

generalized master equation, generalized Langevin equation, operator distribution function, Fokker-Planck equation and average value equation of the relevant organization configuration of physphere

relevant trend parameters and basic vector dynamic equation of interaction situation

For the cosmic ecosphere in the multi-world, the basis of the unified research framework of the nature evolution is summarized as the research of ultra-synergy physics of quantum phy-sphere. This kind of research includes three levels: the study of spin network synergetics for the multi-world ecospheres (quantum-spatiotemporal evolution dynamics), the study of natural paradigm synergetics for high-dimensional supergravity, and the study of quantum phy-sphere synergetics for the cosmic creation. This kind of research is not only self consistent in theory, but also can alleviate the above problems. For the study of how to achieve large scale ratio and spectral index run, refer to [81] - [84] .

In the analysis of this series of papers, as a variable of spin network mode, spatiotemporal mode variable is a vector composed of a series of variables. These variables include:

dimension, curvature, range, expansion rate, dynamicity, quantization, distribution (inhomogeneity, concentration, fluctuation), synergy (cooperation and competition), and so on.

In the quantum phy-spheres studied in this series, the basic unit of matter is the quantum gravi-pulsator. As the smallest condensation of energy, the quantum gravi-pulsator is mainly manifested in the $10^{-44} \mathrm{sec}-10^{-35} \mathrm{sec}$ of the creation of the universe. It is neither a punctiform particle nor a superstring, supermembrane or superembryo. It can be inferred that in the extreme background and 
environmental conditions of the universe, through the big rip-rebound cluster of quantum chaos-reticulate distribution emerged between the huge outwards-push (the Big Bang force, the inflationary force and the expansive force) and the huge inwards-pull (gravitation, strong force, electromagnetic force and weak force), the basic unit of matter must have undergone three basic forms successively: complete chaotic pulsator, quasi-spherical pulsator and spherical pulsator. Various pulse modes of the quantum gravi-pulsator give rise to a variety of elementary particles.

In the quantum phy-spheres studied in this series, boiling at ultra-high temperature is everywhere, and many vacuum bubbles are scattered. At this moment, pseudovacuum decays into the real vacuum, the grand unified symmetry is broken, and there is a synergistic derivation mechanism of the initial cosmic interaction system, so there is the possibility to reveal Higgs effects in a more natural way.

In the model of the super-inflating universe with a great impetus, the Higgs field becomes the origin of vacuum energy in quantum mechanics and then the origin of stationary mass under the great impetus of super-synergy.

In the cosmic inflation, the energy density of the inflationary field is almost unchanged, while the Inhomogeneity, anisotropy, spatial curvature and the number density of various singular particles will be reduced, and the number density of photons will also be reduced, and after enough inflation, it will be reduced to a negligible level. As a result, a flat-symmetrical universe near vacuum was created, which entered reheating.

In the expansion of the universe, the energy density decreases as the volume increases, while the density and volume of ordinary "cold" materials are inversely proportional, the energy density is inversely proportional to the cubic power of the volume, and the radiation energy is inversely proportional to the cubic power of the volume.

After the Big Bang, there will be some remnants, such as microwave background radiation, reflecting small fluctuations of temperature long after the Big Bang (380000 years); for example, there will be adiabaticity, Gaussian density distribution, scaling invariance of density fluctuations, where there is polarization distribution (E mode and B mode), which reveals the statistical information of the universe.

Now, on the basis of various cosmic model analyses, we have found a great impetus with first suppression, later acceleration and last deceleration on the inflation of the universe, from the ultra-synergy (emergence of big rip-rebound clusters with quantum chaotic-reticulate distribution ) between the cosmic outwards-push caused by the Higgs field and the quantum gravity with the initial gravity just separated from the high-dimensional super-gravity, and between the cosmic outwards-push caused by the Higgs field and the initial strong force separated from the super-symmetric grand unified force, thus we have really found the explanation why the universe expands at a critical rate and has the same temperature in different regions.

\section{Repulsion and Gravitation behind Cosmic Inflation}

For the big bang, inflation and subsequent expansion of the universe, we can't help but think of dark energy. For the researchers of dark energy, they often think of the cosmological constant in Einstein's field equation ${ }^{[85]-[87]}$. For cosmological constants, there has always been a so-called fine tuning problem. 
It is generally accepted that the vacuum energy is equivalent to the cosmological constant in physical effect ${ }^{[85-[87]}$. In astronomical observation, if vacuum energy exists, it should always be less than the critical density of the universe, that is:

$$
\rho_{\text {vac }}^{\text {obs }} \leq \rho_{c} \approx 10^{-47} \mathrm{GeV} V^{4}
$$

Through some estimates, the ground state energy (quantum zero point energy) density of a free scalar field with mass $m$ is preliminarily obtained:

$$
\rho_{v a c}^{\text {th }}=\frac{1}{(2 \pi)^{3}} \int_{0}^{k_{c}} \frac{1}{2} \sqrt{k^{2}+m^{2}} 4 \pi k^{2} d k \approx \frac{1}{16 \pi^{2}} k_{c}^{4}
$$

here, $k_{c}$ represents the upper limit of the frequency. If the relevant theory is applicable to Planck energy scale $m_{p} \sim 10{ }^{19} \mathrm{GeV}$, then the density value of vacuum energy can be estimated as: $\rho_{v a c}^{\text {th }} \sim 10^{74} \mathrm{GeV}^{4}$. It is not difficult to find that the observed value of vacuum energy is 121 orders of magnitude smaller than the theoretical value.

For cosmological constants, there are also so-called cosmic coincidence problems. In order to effectively alleviate the fine regulation brought by the coincidence problem in the universe, some researchers have considered the scalar field $\phi$ and the specific potential energy $V(\phi)$ in the energy -moment tensor. Here, the Lagrangian of a scalar field $\phi$ is

$$
S=\int d^{4} x \sqrt{-g}\left[\frac{\varepsilon}{2} \partial_{\mu} \phi \partial^{\mu} \phi-V(\phi)\right]
$$

where, $V(\phi)$ is the potential of scalar field, when $\varepsilon=1$, it means the quantum scalar field; when $\varepsilon=-1$, it means the phantom scalar field. For the variation of action ( 6 ), the energy-moment tensor of scalar field can be obtained:

$$
T^{\mu v}(\phi)=\varepsilon \partial^{\mu} \phi \partial^{v} \phi-g^{\mu \nu}\left[\frac{\varepsilon}{2} \partial_{\mu} \phi \partial^{\mu} \phi-V(\phi)\right]
$$

Both the huge magnitude gap problem in the fine regulation of vacuum energy and the potential energy problem in the fine regulation of cosmic coincidence, they all suggest to us on the other hand that at the beginning of the creation of the universe, the huge magnitude gap contains huge repulsive energy. The quantum repulsion must be closely related to the high-dimensional superrepulsion, and the quantum gravity must be closely related to the high-dimensional supergravity. The quantum conjugate relation is formed spontaneously between the quantum repulsion and the quantum gravity, and the arche-conjugate relation is formed spontaneously between the high-dimensional super repulsion and the high-dimensional super gravity. We can reduce the pseudo vacuum energy considered in inflationary universe model, Higgs field predicted by the standard physical model and dark energy inferred by the observation cosmology to the positive energy system with strong negative pressure, and they can also be reduced to the repulsive force (all factors resistant to the gravity) which forms the conjugate relationship with the gravity.

For the inflationary universe, the repulsion can be theoretically realized by the kinetic energy term of the inflationary field, which is the known k-inflation skyrocketing model ${ }^{[12]-[13]}$. In the period of the 
inflation, the energy of the universe is very high, and the effect of gravity is relatively large. By general relativity, gravity is described by the geometry of space-time. Now, we research the k-inflation model in noncommutative space-time based on the uncertainty principle of space-time. The results show that, in this model ${ }^{[39][40]}$, all disturbance modes are generated in the horizon, and the non commutative effect of space-time contributes to the power spectrum index of disturbance in the form of linear term. Compared with the experimental observation, it is found that the model can better meet the latest data.

Relevant research shows that ${ }^{[25][88]}$, the reason why large-scale structure was formed in the middle and late stage of the universe is that during the period of inflation, the quantum perturbations of the inflation field (also known as the inflation oscillator) and the spatiotemporal background field (known as the gauge field) were rapidly stretched out of the horizon and stopped evolving, and then evolved into classic perturbations and were preserved, and finally became the seed of the formation of large-scale structure. In fact, the inflation may never end, which is called the eternal inflation ${ }^{[88]-[90]}$. In many experiments, detecting the B-mode of photon polarization has been taken as the primary task. It is shown that only tensor perturbation can contribute to B-mode, while scalar perturbation only contributes to E-mode. Detection of B-mode is equivalent to detection of tensor disturbance, or detection of primary gravitational wave. The well-known BICEP2 project is based on their detection of disturbed B-mode. The data shows that ${ }^{[73][37]}$, the tensor-scale ratio is limited to $r=0.20_{-0.05}^{+0.07}$ and the possibility of $r=0$ is excluded at the confidence level of $7.0 \sigma$. Based on these data, we can limit and exclude all kinds of the inflation models, including simple chaos inflation to complex multi-field models.

For the inflation of the commutative universe, some studies consider that a complex kinetic energy term is called irregular kinetic energy, and such a model is called K-inflation ${ }^{[25][88]-[90]}$. For example, Tachyon model ${ }^{[4][20][91]}$, Dirac-born-Infeld model ${ }^{[15][21]}$, all belong to this category.

At the time of the inflation, the energy scale of the universe is very high, which can be compared with the grand unification or even the Planck energy scale. Therefore, when it comes to the calculation of the inflation field, some appropriate corrections from quantum gravity are needed. As one of the most promising candidates of quantum gravity, superstring theory should give the necessary correction methods. In fact, nonperturbative superstring (or $\mathrm{m}$ ) theory points out that any physical process, when the interaction distance is very small, should meet the uncertainty relationship: $\Delta t_{p} \Delta x_{p} \geq l_{s}^{2}$.

Now, we consider introducing the interaction of repulsion and gravitation into the inflation model in noncommutative space-time.

First, consider the repulsion field behind the inflation. if the kinetic energy term of a single scalar field $\varphi$ is expressed as $X=-\frac{1}{2} g^{\mu \nu} \nabla_{\mu} \varphi \nabla_{\nu} \varphi$, then the most general repulsive action used to describe the field can be expressed as:

$$
S_{\varphi}=\int d^{4} X \sqrt{-g}\left[\frac{R}{2}+p_{r e p l}(\varphi, X)\right],
$$

here $p_{\text {repl }}(\varphi, X)$ is an arbitrary function of the scalar field $\varphi$ itself and its kinetic energy, and the definition of $X$ and the above repulsive action ensure that the motion equation of the field is second 
order. The energy momentum tensor of the inflation field can be obtained by the variation of the repulsion action.

$$
\begin{gathered}
K_{\mu \nu} \equiv-\frac{2}{\sqrt{-g}} \frac{\delta S_{\varphi}}{\delta g^{\mu \nu}}=p_{r e p l, X} \nabla_{\mu} \varphi \nabla_{\nu} \varphi+p_{r e p l} g_{\mu \nu}, \\
p_{r e p l, X} \equiv \frac{\partial p_{r e p l}(\varphi, X)}{\partial X},
\end{gathered}
$$

It is also equal to an ideal fluid, as long as its energy-momentum tensor is expressed as

$$
K_{\mu v}=\left(\sigma+p_{r e p l}\right) U_{\varphi, \mu} U_{\varphi, v}+p_{r e p l} g_{\mu v} .
$$

The $p_{\text {repl }}$ appearing here is the pressure $\left(p_{\text {repl }}<0\right)$ defined of repulsion in the co-moving coordinate system, while the energy density defined in the same coordinate system is:

$$
\sigma=2 X p_{r e p l, X}-p_{r e p l}
$$

In addition, the definition of four velocity $U_{\varphi, \mu}$ is $U_{\varphi, \mu}=-\nabla_{\mu} \varphi / \sqrt{2 X}$.

Second, consider the gravitational field behind the inflation. if the kinetic energy term of a tensor field $\theta$ is expressed as $x=-\frac{1}{2} g^{\mu \nu} \nabla_{\mu} \theta \nabla_{\nu} \theta$, then the most general gravitation action used to describe the field can be expressed as:

$$
S_{\theta}=\int d^{4} x \frac{1}{\sqrt{-g}}\left[\frac{2}{R}+p_{\text {grav }}(\theta, x)\right],
$$

here $p_{\text {grav }}(\theta, x)$ is an arbitrary function of the tensor field $\varphi$ itself and its kinetic energy, and the definition of $x$ and the above gravitational action ensure that the motion equation of the field is second order. The energy momentum tensor of the inflation field can be obtained by the variation of the gravitational action.

$$
\begin{gathered}
T_{\mu \nu} \equiv-\frac{1}{2} \sqrt{-g} \frac{\delta S_{\theta}}{\delta g^{\mu \nu}}=p_{\text {grav }, X} \nabla_{\mu} \theta \nabla_{\nu} \theta+p_{\text {grav }} g_{\mu \nu}, \\
p_{\text {grav }, X} \equiv \frac{\partial p_{\text {grav }}(\theta, x)}{\partial x},
\end{gathered}
$$

It is also equal to an ideal fluid, as long as its energy-momentum tensor is expressed as

$$
T_{\mu v}=\left(\rho+p_{\text {grav }}\right) U_{\theta, \mu} U_{\theta, v}+p_{\text {grav }} g_{\mu \nu} .
$$

The $p_{\text {grav }}$ appearing here is the pressure ( $p_{\text {grav }}>0$ ) of gravity defined in the co-moving coordinate system, while the energy density defined in the same coordinate system is:

$$
\rho=2 x_{\text {grav }, x}-p_{\text {grav }} .
$$


In addition, the definition of four velocity $U_{\theta, \mu}$ is $U_{\theta, \mu}=-\nabla_{\mu} \theta / \sqrt{2 x}$.

Now, consider the interaction between the repulsive field and the gravitational field behind the inflation. In this case, the most general hedging action between the repulsive field and the gravitational field behind the inflation field can be expressed as follows:

$$
S_{\varphi}-S_{\theta}=\int d^{4} X \sqrt{-g}\left[\frac{R}{2}+p_{\text {repl }}(\varphi, X)\right]-\int d^{4} x \frac{1}{\sqrt{-g}}\left[\frac{2}{R}+p_{\text {grav }}(\theta, x)\right]=\pi_{s},
$$

where, $\pi_{s} \gg 0$.

By the variation of the action, the energy momentum tensor of the hedging action behind the inflation field can be obtained

$$
\begin{aligned}
K_{\mu \nu}-T_{\mu \nu} \equiv & -\frac{2}{\sqrt{-g}} \frac{\delta S_{\varphi}}{\delta g^{\mu \nu}}+\frac{1}{2} \sqrt{-g} \frac{\delta S_{\theta}}{\delta g^{\mu \nu}} \\
= & p_{\text {repl }, X} \nabla_{\mu} \varphi \nabla_{\nu} \varphi+p_{\text {repl }} g_{\mu \nu}-p_{\text {grav }, X} \nabla_{\mu} \theta \nabla_{\nu} \theta-p_{\text {grav }} g_{\mu \nu} \\
& p_{\text {repl }, X}-p_{\text {grav }, x} \equiv \frac{\partial p_{\text {repl }}(\varphi, X)}{\partial X}-\frac{\partial p_{\text {grav }}(\theta, x)}{\partial x}
\end{aligned}
$$

It is equivalent to a fluid, as long as its hedging energy momentum tensor is expressed as

$$
K_{\mu v}-T_{\mu \nu}=\left(\sigma+p_{\text {repl }}\right) U_{\varphi, \mu} U_{\varphi, v}+p_{\text {repl }} g_{\mu \nu}-\left(\rho+p_{\text {grav }}\right) U_{\theta, \mu} U_{\theta, v}-p_{\text {grav }} g_{\mu \nu} .
$$

For an uniform and homogeneous flat universe ( space curvature $K=0$ ) can be described by Friedmann-Robertson-Walker (FRW) gauge in the form of $d s^{2}=-d t^{2}+a^{2}(t) d x^{2}$. In this way, the kinetic energy

$$
X-x=\frac{1}{2}\left(\dot{\varphi}^{2}-\dot{\theta}^{2}\right),
$$

of the inflation field and the noncommutative relation of time and space become: $\Delta t \Delta x \geq l_{s}^{2} / a(t)$.

In order to make the non commutative relation meaningful at any time, we must introduce a new time coordinate $\tau: d \tau=a(t) d t$, the gauge also changes. Now, there is a good definition of non commutative relation of time and space: $\Delta \tau \Delta x \geq l_{s}^{2}$.

By introducing FRW gauge into the repulsive field equation in the inflation, non Friedmann equation related to repulsive field can be obtained:

$$
H_{r e p l}^{2}=\frac{\sigma}{3}=\frac{1}{3}\left(\dot{\varphi}^{2} p_{r e p l, x}-p_{\text {repl }}\right)
$$

and by introducing FRW gauge into the gravitational field equation in the inflation, Friedmann equation 
related to gravitational field can be obtained:

$$
H_{\text {grav }}^{2}=\frac{\rho}{3}=\frac{1}{3}\left(\dot{\theta}^{2} p_{\text {grav }, X}-p_{\text {grav }}\right),
$$

Thus we can set up the hedge-expansive universe equation reflecting the interaction between repulsion and gravity:

$$
H_{\text {repl }}^{2}-H_{\text {grav }}^{2}=\frac{1}{3}(\sigma-\rho)=\frac{1}{3}\left(\dot{\varphi}^{2} p_{\text {repl }, x}-p_{\text {repl }}-\dot{\theta}^{2} p_{\text {grav }, X}+p_{\text {grav }}\right) .
$$

By the variation of the repulsion action ( 8 ), the equation of motion related to repulsion in the inflation field can be obtained

$$
\ddot{\varphi}\left(p_{r e p l, X}+\dot{\varphi}^{2} p_{r e p l, X X}\right)+3 H_{r e p l} \dot{\varphi} p_{r e p l, X}=p_{r e p l, \varphi}-\dot{\varphi}^{2} p_{r e p l, X \varphi} .
$$

In addition, there are the equation

$$
\dot{H}_{r e p l}=-X p_{r e p l, X}=-\frac{1}{2} \dot{\varphi}^{2} p_{r e p l, X}
$$

Because of the irregular kinetic energy term in function $p_{\text {repl }}$, the dispersion relation of the inflation field is modified, and the perturbation mode of quantum repulsion no longer moves at the speed of light. The propagation velocity of repulsive disturbance can be characterized by the velocity of sound defined by the following formula:

$$
c_{s, r e p l}^{2} \equiv \frac{p_{\text {repl }, X}}{\sigma_{X}}=\frac{p_{\text {repl }, X}}{p_{r e p l, X}+\dot{\varphi}^{2} p_{r e p l, X X}} \leq 1
$$

Back to the regular case: $p_{\text {repl }}(\varphi, X)=X-V(\varphi), V(\varphi)$ is the repulsive potential energy of the inflation field.

By the variation of the gravitational action ( 13 ), the equation of motion related to gravitation in the inflation field can be obtained

$$
\ddot{\theta}\left(p_{\text {grav }, x}+\dot{\theta}^{2} p_{\text {grav }, x x}\right)+3 H_{\text {grav }} \dot{\theta} p_{\text {grav }, x}=p_{\text {grav }, \varphi}-\dot{\theta}^{2} p_{\text {grav }, x \varphi} .
$$

In addition, there are the equation

$$
\dot{H}_{\text {grav }}=-x p_{\text {grav }, x}=-\frac{1}{2} \dot{\theta}^{2} p_{\text {grav }, x}
$$

Because of the irregular kinetic energy term in function $p_{\text {grav }}$, the dispersion relation of the inflation field is modified, and the perturbation mode of quantum gravitation no longer moves at the speed of light. The propagation velocity of gravitational disturbance can be characterized by the velocity of sound defined by the following formula:

$$
c_{s, \text { grav }}^{2} \equiv \frac{p_{\text {grav }, x}}{\rho_{x}}=\frac{p_{\text {grav }, x}}{p_{\text {grav }, x}+\dot{\theta}^{2} p_{\text {grav }, x x}} \leq 1 .
$$

Back to the regular case: $p_{\text {grav }}(\theta, x)=x-V(\theta), V(\theta)$ is the gravitational potential energy of the inflation field. 
By combining equation ( 26 ) with equation ( 28 ), the motion equation of the hedging between repulsion and gravitation in the inflation field can be obtained

$$
\begin{gathered}
\ddot{\varphi}\left(p_{\text {repl }, X}+\dot{\varphi}^{2} p_{\text {repl }, X X}\right)-\ddot{\theta}\left(p_{\text {grav }, x}+\dot{\theta}^{2} p_{\text {grav }, x x}\right)+3\left(H_{\text {repl }} \dot{\varphi} p_{\text {repl }, X}-H_{\text {grav }} \dot{\theta} p_{\text {grav }, x}\right) \\
=p_{\text {repl }, \varphi}-\dot{\varphi}^{2} p_{\text {repl }, X \varphi}-p_{\text {grav }, \varphi}+\dot{\theta}^{2} p_{\text {grav }, X \varphi}
\end{gathered}
$$

In addition, there is

$$
\dot{H}_{r e p l}-\dot{H}_{g r a v}=-X p_{r e p l, X}-x p_{g r a v, x}=-\frac{1}{2} \dot{\varphi}^{2} p_{r e p l, X}+\frac{1}{2} \dot{\theta}^{2} p_{g r a v, x}
$$

The propagation velocity of hedging disturbance can be characterized by the velocity of sound defined by the following formula:

$$
c_{s, \text { repl }}^{2}-c_{s, \text { grav }}^{2}=\frac{p_{\text {repl }, X}}{\sigma_{X}}-\frac{p_{\text {grav }, x}}{\rho_{x}}=\frac{p_{\text {repl }, X}}{p_{\text {repl }, X}+\dot{\varphi}^{2} p_{\text {repl }, X X}}-\frac{p_{\text {grav }, x}}{p_{\text {grav }, x}+\dot{\theta}^{2} p_{\text {grav }, x x}} \leq 1 .
$$

Back to the regular case:

$$
p_{\text {repl }}(\varphi, X)-p_{\text {grav }}(\theta, x)=X-x-V(\varphi)+V(\theta),
$$

$V(\varphi)-V(\theta)$ is the hedge-potential energy of the inflation field, the speed of sound is equal to $c_{s, \text { repl }}^{2}-c_{s, \text { grav }}^{2}=1$.

Therefore, we can define several slow-rolling parameters to describe the process of the inflation:

$$
\begin{gathered}
\varepsilon_{1}=-\frac{\dot{H}_{\text {repl }}}{H_{\text {repl }}^{2}}+\frac{\dot{H}_{\text {grav }}}{H_{\text {grav }}^{2}}=\frac{X p_{\text {repl }, X}}{H_{\text {repl }}^{2}}-\frac{x p_{\text {grav }, x}}{H_{\text {grav }}^{2}}, \\
\varepsilon_{2}=\frac{\dot{\varepsilon}_{1}}{\varepsilon_{1}\left(H_{\text {repl }}-H_{\text {grav }}\right)}, \\
s=\frac{\dot{c}_{s, \text { repl }}-\dot{c}_{s, \text { grav }}}{c_{s, \text { repl }} H_{\text {repl }}-c_{s, \text { grav }} H_{\text {grav }}},
\end{gathered}
$$

They are small in the period of the inflation, i.e. $\left|\varepsilon_{1}\right|,\left|\varepsilon_{2}\right|, s \leq 1$.

It can be inferred that in the early evolution of the universe, the interaction between quantum repulsion and quantum gravity led to a big rip and a big rebound. Then it is concluded that in the initial stage of the big rip, quantum repulsion and quantum gravity restrain each other, leading to the slowing down of the big bang of the universe; and at the end of the big rip, quantum repulsion and quantum gravity form a superposed big rebound, leading to the super inflation of the universe.

\section{Perturbation of Repulsive Scalar and Gravitational Tensor}

On the one hand, the scalar disturbance is considered as the showing of repulsive effect, on the other hand, the tensor disturbance is considered as the showing of gravitational effect. In the initial stage of the big rip, due to the mutual restraint of quantum repulsion and quantum gravity, the scalar 
and tensor perturbations are not obvious. However, at the end of the big rip, the scalar and tensor perturbations are very significant, and there is a large power spectral exponential run.

Let $V$ be the volume of space, and the repulsive action of scalar disturbance can be given by

$$
S_{\varphi}=\frac{V}{2} \int_{k<k_{0}} d \eta d^{3} k \approx z^{2}(\eta)\left(\zeta_{-k}^{\prime} \zeta_{k}^{\prime}-c_{s, r e p l}^{2} k^{2} \zeta_{-k} \zeta_{k}\right),
$$

where the apostrophe represents the differential of the new time coordinate $\eta$, which is defined as:

$$
\frac{d \eta}{d \tau} \equiv a_{e f f}^{-2}=\left(\frac{\beta_{k}^{-}}{k_{+}^{\beta}}\right)^{1 / 2}=\frac{1}{a^{2}}(\tau-\Delta \tau)
$$

here the function of $\beta_{k}^{ \pm}(\tau)$ is defined :

$$
\beta_{k}^{ \pm}(\tau)=a^{ \pm 2}(\tau-\Delta \tau), \quad \Delta \tau=l_{s}^{2} k
$$

where $\zeta_{k}$ is the curvature disturbance and $z \equiv a \sqrt{2 \varepsilon_{1}} / c_{s}$ is Mukhanov variable. The $\beta_{k}^{ \pm}(\tau)$ function used here is equivalent to the formula used in other literature in the sense of integral, and once the exact solution of the perturbed equation is obtained, the form of the $\beta_{k}^{ \pm}(\tau)$ function will not affect the final physical results. However, if the present form is chosen, the effect of non commutation of space-time can be highlighted to the greatest extent under the approximate conditions, and the perturbed equation will become easier to deal with. The equation of motion satisfied by the variable $\zeta_{k}$ is:

$$
u_{k}^{\prime \prime}+\left(c_{s}^{2} k^{2}-\frac{z^{\prime \prime}}{z}\right) u_{k}=0
$$

This equation can be obtained by the variation of action ( 34 ), where the modulus function is defined as $u_{k}=z \zeta_{k}$. By using the slow-rolling approximation condition and the slow-rolling parameters, the third coefficient of the disturbance equation ( 37 ) can be obtained:

$$
\frac{z^{\prime \prime}}{z} \approx \frac{1}{\eta^{2}}\left(1-\varepsilon_{1}\right)^{-2} \Sigma^{-2}(\tau, \Delta \tau)\left[2 \Sigma(\tau, \Delta \tau)\left(1+\frac{\varepsilon_{2}}{2}-s\right)-\varepsilon_{1}+\frac{\varepsilon_{2}}{2}-s\right] .
$$

The following relationships are used in the derivation:

$$
\eta \approx-\left[a(\tau-\Delta \tau) H(\tau-\Delta \tau)\left(1-\varepsilon_{1}\right)\right]^{-1},
$$

This relationship can be obtained by equation ( 35 ). In ( 39 ) function $\Sigma$ is defined as:

$$
\Sigma(\tau, \Delta \tau)=\frac{a(\tau)}{a(\tau-\Delta \tau)} \frac{H_{r e p l}(\tau-\Delta \tau)}{H_{r e p l}(\tau)} .
$$

It can be seen from the uncertainty principle of time and space that the time when all modes are generated is when the non-commutative relation of time and space takes the equal sign. At this moment, the co-moving wave number has the maximum value: 


$$
k_{0}(\tau)=\frac{a_{e f f}}{l_{s}}=\frac{a\left(\tau+\Delta_{\tau}\right)}{l_{s}} \approx \frac{H_{r e p l}(\tau)}{l_{s}}\left[a(\tau) H_{r e p l}^{-1}(\tau)-\Delta \tau\right]
$$

In other words, at time $\tau$, a mode of wave number to be $k_{0}$ is generated. Moreover, the value of $\lambda$ at this time can be estimated:

$$
\left.\lambda_{0} \equiv \frac{\Delta \tau}{a(\tau) H_{r e p l}^{-1}(\tau)-\Delta \tau}\right|_{k=k_{0}} \approx l_{s} H_{*}
$$

where $H_{*}^{-1}$ is called the Hubble horizon. In the period of the inflation, the scale factor $a(t)$ either evolves in the form of approximate exponential function, i.e. $a \sim e^{H t}$, or in the form of power-law function, i.e. $a \sim t^{n}$, where $n$ is a relatively large constant. At the same time, $H *$ hardly evolves. Thus, by equation ( 42 ), it can be seen that the parameter $\lambda$ decreases with time.

At time $\tau$, if a mode just crosses the horizon, it should have momentum

$$
k_{c}=a(\tau) \frac{H_{r e p l}(\tau)}{c_{s, r e p l}}
$$

then we can get:

$$
\frac{k_{c}}{k_{0}} \approx \frac{a(\tau)}{a(\tau)-H_{r e p l}(\tau) \Delta \tau} \frac{l_{s} H_{r e p l}(\tau)}{c_{s, r e p l}} \approx \frac{l_{s} H_{*}}{c_{s, r e p l}}
$$

In the following, the research will focus on the case of $l_{s} H * / c_{s, \text { repl }} \ll<1$, that is, all the modes are generated within the horizon $\left(k_{0} \gg>k_{c}\right)$. The reason for not considering another case $l_{s} H * / c_{s, \text { repl }} \geq 1$ is that it is difficult to explain the flatness of microwave background radiation. See literature [90] [88] for detailed discussion. Therefore, in the case of $l_{s} H_{*} / c_{s, \text { repl }} \ll<1$, it is very reasonable to treat $\lambda$ as a small quantity and retain its first-order term in the calculation. In this way, the power spectrum of the disturbance can be calculated at the time when the mode is out of the horizon $\left(k=a H_{\text {repl }} / c_{s, \text { repl }}\right)$.

In the case of slow-roll approximation, equation ( 37 ) is simplified to:

$$
u_{k}^{\prime \prime}+\left(c_{s, r e p l}^{2} k^{2}-\frac{v^{2}-1 / 4}{\eta^{2}}\right) u_{k}=0
$$

Take the initial condition as the Bunch-Davies vacuum defined as follows: $u_{k}=\frac{1}{\sqrt{2 c_{s, \text { repl }} k}} e^{-i c_{s, r e p l} k \eta}$.

The final solution of equation ( 45 ) is as follows:

$$
u_{k}(\eta)=\frac{\sqrt{\pi}}{2} e^{i(v+1 / 2) \pi / 2} \sqrt{-\eta} H_{v, \text { repl }}^{(1)}\left(-c_{s, r e p l} k \eta\right)
$$

where, $H_{v, \text { repl }}^{(1)}$ represents the first kind of Hankel function related to repulsion. On the scale beyond the horizon, the solution is approximately: 


$$
u_{k}(\eta)=2^{\nu-3 / 2} e^{i(v-1 / 2) \pi / 2} \frac{\Gamma(v)}{\Gamma(3 / 2)} \frac{1}{\sqrt{2 c_{s, r e p l} k}}\left(-c_{s, r e p l} k \eta\right)^{1 / 2-v},
$$

Then, the original scalar perturbation spectrum is obtained:

$$
\begin{aligned}
P_{s} & =\frac{k^{3}}{2 \pi^{2}}\left|\zeta_{k}\right|^{2}=\frac{k^{3}}{2 \pi^{2}}\left|\frac{u_{k}}{z}\right|^{2} \\
& =\left.\frac{2^{2 v-4}}{\varepsilon_{1} c_{s, r e p l}}\left[\frac{\Gamma(v)}{\Gamma(3 / 2)}\right]^{2}\left(\frac{H}{2 \pi}\right)^{2}\left(\frac{c_{s, r e p l} k}{a H_{\text {repl }}}\right)^{3-2 v}\right|_{c_{s, r e p l} k=a H_{r e p l}} \approx \frac{1}{8 \pi^{2} \varepsilon_{1} c_{s, r e p l}} \frac{H_{r e p l}^{2}}{M_{p l}^{2}} c_{s, \text { repl }}^{2} k a H_{\text {repl }}
\end{aligned}
$$

By the definition of the scalar disturbance spectrum index, we can get:

$$
n_{s}-1 \equiv \frac{d \ln P_{s}}{d \ln k}=3-2 v-s \approx-2 \varepsilon_{1}-\varepsilon_{2}+s+\frac{4}{3} \lambda,
$$

In addition, the spectral index runs as follows:

$$
\alpha_{s} \equiv \frac{d n_{s}}{d \ln k}=-2 \varepsilon_{2} \varepsilon_{1}-\varepsilon_{3} \varepsilon_{2}+s_{1} s-\frac{4}{3} \lambda(1+\lambda)\left(1+\varepsilon_{1}\right) \approx-\frac{4}{3} \lambda .
$$

Some small quantities $\varepsilon_{3} \equiv \dot{\varepsilon}_{2} /\left(\varepsilon_{2} H_{\text {repl }}\right)$ and $s_{1} \equiv \dot{s} /\left(s H_{\text {repl }}\right)$ are defined here.

The motion equation of tensor perturbation related to repulsion is almost the same as that of scalar perturbation, but the definition of Mukhanov variable becomes $z=a$. The specific disturbance equation is as follows:

$$
v_{k}^{\prime \prime}+\left(k^{2}-\frac{a^{\prime \prime}}{a}\right) v_{k}=0,
$$

where the modular function is defined as $v_{k} \equiv a h k / 2$. It should be noted that $h_{k}$ here represents two physical freedom degrees of tensor perturbation, namely $h_{+}$and $h_{x}$. Using the same approximation as scalar perturbation, we get:

$$
\frac{a^{\prime \prime}}{a} \approx \frac{1}{\eta^{2}}\left(1-\varepsilon_{1}\right)^{-2} \Sigma^{-2}(\tau, \Delta \tau)\left[2 \Sigma(\tau, \Delta \tau)-\varepsilon_{1}\right] \approx \frac{v^{2}-1 / 4}{\eta^{2}},
$$

The solution of equation ( 51 ) is obtained by keeping the value of $v$ to the linear term:

$$
v_{k}(\eta)=\frac{\sqrt{\pi}}{2} e^{i(v+1 / 2) \pi / 2} \sqrt{-\eta} H_{v, r e p l}^{(1)}(-k \eta) .
$$

The solution also satisfies the Bunch-Davies vacuum condition. On the scale beyond the horizon, the solution is approximately:

$$
u_{k}(\eta)=2^{v-3 / 2} e^{i(v-1 / 2) \pi / 2} \frac{\Gamma(v)}{\Gamma(3 / 2)} \frac{1}{\sqrt{2 k}}(-k \eta)^{1 / 2-v},
$$

then, the original tensor perturbation spectrum related to repulsion is obtained. 


$$
\begin{aligned}
P_{t} & =2 \times \frac{k^{3}}{2 \pi^{2}}\left|h_{k}\right|^{2}=\frac{k^{3}}{\pi^{2}}\left|\frac{2 v_{k}}{z}\right|^{2} \\
& =\left.\left.2^{2 v}\left[\frac{\Gamma(v)}{\Gamma(3 / 2)}\right]^{2}\left(\frac{H_{\text {grav }}}{2 \pi}\right)^{2}\left(\frac{k}{a H_{\text {grav }}}\right)^{3-2 v}\right|_{k=a H_{\text {grav }}} \approx \frac{2}{\pi^{2}} \frac{H_{\text {grav }}^{2}}{M_{p l}^{2}}\right|_{k=a H_{\text {grav }}} .
\end{aligned}
$$

By the definition of tensor disturbance spectrum index and tensor-scale ratio, we can get:

$$
n_{t} \equiv \frac{d \ln P_{t}}{d \ln k}=3-2 v \approx-2 \varepsilon_{1}+\frac{4}{3} \lambda, \quad r \equiv \frac{P_{t}}{P_{s}}=16 c_{s, r e p l} \varepsilon_{1} .
$$

It is precisely because the $\lambda$ term appears in the above equation that a new kind of inflationary universe model with kinetic energy term based on the non commutative relation of space-time will be more consistent with the experimental observation than the related model in the commutative space-time. Next, by references [36][37][73], we can compare and analyze these models with the latest experimental observations.

In order to constrain the parameters (fast sub model and DBI model) in the K-inflation model in non commutative space-time, the latest observation data are used, including: microwave background radiation data in Planck experiment, supplemented by large-scale polarization data in WMAP ( hereinafter referred to as Planck + WP ); the large polar moment data of microwave background radiation ( hereinafter referred to as high L ); Planck gravitational lens power spectrum. Large scale structure ( hereinafter referred to as BAO ) data, etc. Specific data in reference [36]: scalar spectral index 0. $9583 \pm 0.0081$ ( Planck + WP ), 0. $9633 \pm 0.0072$ ( Planck + WP + lensing ), 0. $9570 \pm 0$. $0075\left(\right.$ Planck + WP + high L ), $0.9607 \pm 0.0063($ Planck $+\mathrm{WP}+\mathrm{BAO})$, its running value $n{ }^{\prime}=-0$. $021 \pm 0.012($ Planck + WP $), n{ }_{s}=-0.017 \pm 0.012($ Planck $+\mathrm{WP}+$ lensing $), n_{s}^{\prime}=-0.022_{-0.010}^{+0.011}$ $($ Planck $+\mathrm{WP}+$ high L $), n_{s}^{\prime}=-0.021_{-0.010}^{+0.012}($ Planck $+\mathrm{WP}+\mathrm{BAO})$; and in reference [37]: tensor -scale ratio $r=0.20_{-0.05}^{+0.07}$. In addition, there are values given in reference [73]: $r=0.23_{-0.09}^{+0.05}$ and $n_{t}=0.03_{-0.11}^{+0.13}$.

For the inflation of tachyon, considering the case that $\mathrm{M}$ is large enough, and taking the e-folding number as a parameter, the following limitations are obtained by fitting:

$$
\lambda=0.0138_{-0.00409}^{+0.00404}, \quad N=34.64_{-4.149}^{+5.489} \quad(68 \% \mathrm{CL}) .
$$

For the DBI inflation model, the parameters are limited to:

$$
\lambda=0.0998_{-0.00368}^{+0.00363}, \quad N=59.61_{-7.287}^{+9.692} \quad(68 \% \mathrm{CL}) .
$$

Figure 2 provided in reference [37] shows the contour of parameters in the confidence interval of $1 \sigma$ and $2 \sigma$. It can be seen from Figure 7 that the e-folding number in the tachyon model is relatively small. If the value of e-folding number is required to be between 50 and 60, the model can only be said to be in good accordance with the observation; however, in the DBI model, the value of e-folding 
number is enough to solve the flatness problem in the big bang cosmology.
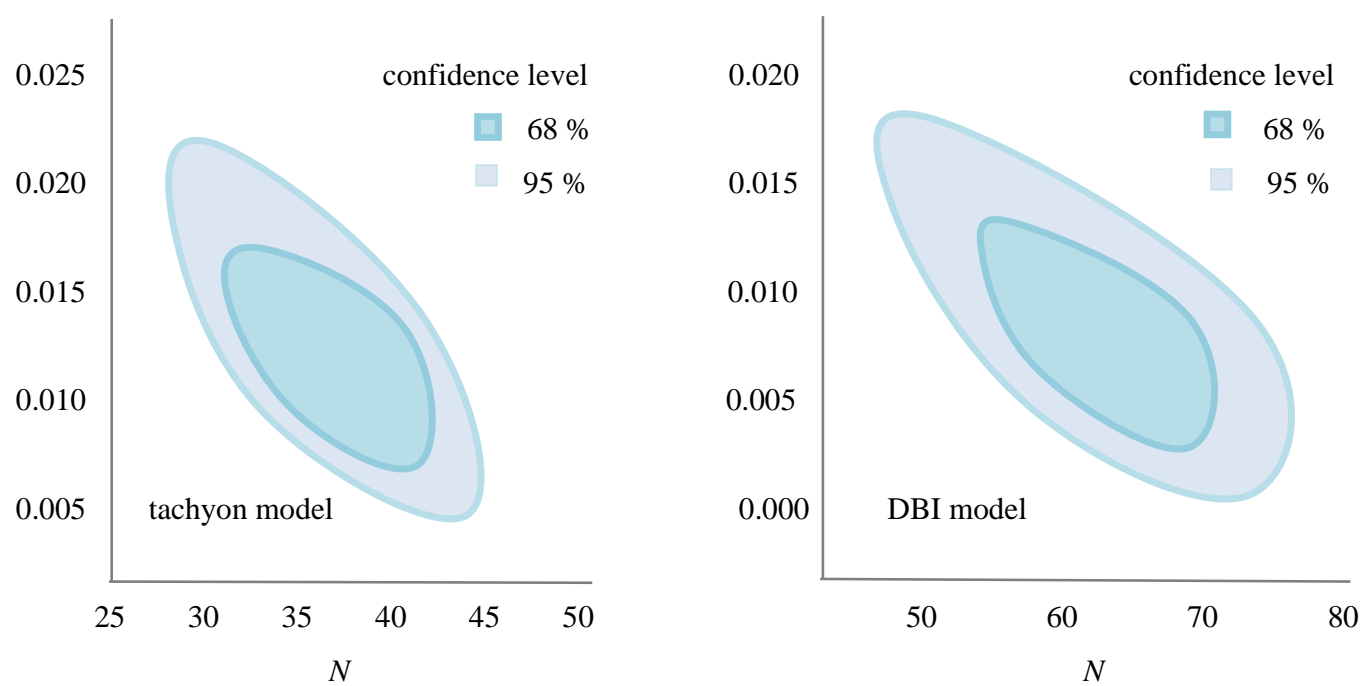

The center red dot represents the best fit value of the parameter.

Here, the left figure corresponds to the tachyon model; the right figure corresponds to the DBI model.

Fig. 2 contour of parameters $\lambda$ and $N$ in confidence intervals $1 \sigma$ and $2 \sigma$

It can be seen from the above discussion that repulsion and gravitation always interact in the big bang, inflation and subsequent expansion of the universe.

\section{Model (I) of Super-Inflating Universe with Big Impetus}

Early studies of the inflationary universe assumed that the Hggs field, which caused the break of the grand unified symmetry, drove the inflation ${ }^{[1][77][31]}$. However, it was soon found that the effective potential of the Higgs field was not flat enough due to the radiation correction given by the gauge coupling, and that the quantum fluctuation of the Higgs field would give too large and unacceptable mass density fluctuations ${ }^{[5][50][55]}$. Therefore, in the later model, scalar field $\varphi$ is a gauge singlet, called an expander. In most cases, this field only promotes inflation, and all models based on slow roll phase transition are called new inflation cosmic models ${ }^{[50][55][56]}$.

The new inflation cosmic model set by Andrei Linde, Andreas Albrecht and Paul Steinhardt, et al. is basically consistent with the initial conditions of the universe in the original model of inflationary universe ${ }^{[50][55][56]}$. In order to obtain a new inflationary cosmological model, Linde, Albrecht and Steinhardt et al. require particle physics theory to contain a scalar field $\varphi$, and have the following properties $^{[50][55][56]}$ : (i) the effective potential function $V(\varphi)$ must have a minimum at $\varphi \neq 0$; (ii) $V(\varphi)$ must be very flat near $\varphi=0$, and it is usually assumed that at $\varphi=0$ It is a local maximum of $V(\varphi)$; (iii) at high temperature $T$, the heat equilibrium value of $\varphi$, i.e. the minimum of the effective potential $V_{T}$ $(\varphi, T)$ at finite temperature must be at $\varphi=0$.

Now, we are trying to adjust and modify the old and new models of the inflationary universe to relax the strict restrictions of Linde, Albrecht and Steinhardt on a scalar field of particle physics. In the supernatural way of ontology, combining physical geometry with geometric physics in the supernatural 
way of ontology, and then combining physical cosmology with cosmic physics, we study the following ultra-synergy (emergence of big rip-rebound clusters with quantum chaotic-reticulate distribution) between the universe and ever-increasing particles (clusters):

The ultra-synergy (emergence of big rip-rebound clusters with quantum chaotic-reticulate distribution) between the quantum universe in the Big Bang and ever-increasing quantum gravitational sources (clusters),

The ultra-synergy (emergence of big rip-rebound clusters with quantum chaotic-reticulate distribution) beteen the inflationary universe and ever-increasing gravitational sources (clusters) as well as strong force sources (clusters),

The ultra-synergy (emergence of big rip-rebound clusters with quantum chaotic-reticulate distribution) between the expansive universe and ever-increasing gravitational sources (clusters), strong force sources (clusters) and electro-weak force sources (clusters).

These super-synergies in several phase transitions during the creation of the universe are the driving forces leading to the emergence of rip-rebound-inflation in the universe.

By the research in [88], let $V$ represent the potential energy function of the inflation field, and assume that it has the following form:

$$
V(\phi)=\lambda \sum_{n=1}^{\infty} a_{n} \phi^{2 n}
$$

where $\lambda$ is a proportional coefficient, which will be determined by the amplitude of the power spectrum. Define slow roll parameters:

$$
\varepsilon=\frac{M_{p l}^{2}}{2}\left(\frac{V^{\prime}}{V}\right)^{2}, \quad \eta=M_{p l}^{2} \frac{V^{\prime \prime}}{V^{\prime}}, \quad \xi=M_{p l}^{4} \frac{V^{\prime} V^{\prime \prime}}{V^{2}},
$$

where the apostrophe is the differential of the field $\phi$, the power spectrum index $n_{s}$ and its running $n_{s}^{\prime}$ of the scalar disturbance can be expressed as:

$$
n_{s}-1 \approx 2 \eta-6 \varepsilon, \quad n_{s}^{\prime} \approx 16 \varepsilon \eta-24 \varepsilon^{2}-2 \xi .
$$

In fact, any potential energy function can be expanded into infinite power series, for example, the exponential potential energy $V \sim e^{\phi}$, can be written as $V \sim \Sigma \phi^{n} / n !$, and the general potential energy can also be expanded into Taylor series. However, when people expand a general potential function according to Taylor series, they will encounter infinite expansion coefficients, which makes the model impossible to deal with. The only way is to expand the potential energy by several steps when the surge occurs, and then reconstruct the potential energy according to the experimental data, as shown in [93] [94]. In equation (55), although the potential energy has infinite items, there are only three parameters: $\alpha, \beta$ and $\lambda$. Such a potential energy may come from some super gravity theory (sugra):

$$
V=e^{K}\left[K^{i j}\left(D_{i} W\right)\left(D_{W} W\right)-3|W|^{2}\right], \quad D_{i} W=\partial_{i} W+\left(\partial_{i} K\right) W,
$$

Where Kahler potential is taken as: 


$$
K=g_{1}\left(\varphi+\varphi^{\dagger}\right)+\frac{1}{2}\left(\varphi+\varphi^{\dagger}\right)^{2}+|X|^{2}-c_{X}|X|^{4}+\cdots,
$$

(59)

It can be predicted that the theory is shift invariant and the symmetry ensures that the potential energy is flat along the imaginary part of the field $\varphi$. Here $\phi \equiv \sqrt{2} \operatorname{Im} \varphi$ is the inflationary field, and $X$ is another chiral superfield. In addition, it is assumed that the hyperpotential is in the most general case:

$$
W=X \sum h_{n} \phi^{n}+W_{0}
$$

For the theoretical study of super gravity, please refer to [10]. In addition, the polynomial inflation model is considered in references [95] - [96], that is, the potential energy has some finite terms.

It can be seen from Fig. 3 that the potential energy is a monotonic increasing function of the inflation field $\phi$. When the parameter $\alpha$ is small, the potential energy is more steep. It is also obviously different from the case where the chaotic inflation takes the form of $\phi^{2}$ or $\phi^{4}$. When $\phi$ is small, it is close to $\phi^{2}$ potential, but with the increase of $\phi$ value, it is close gradually to $\phi^{4}$ potential.
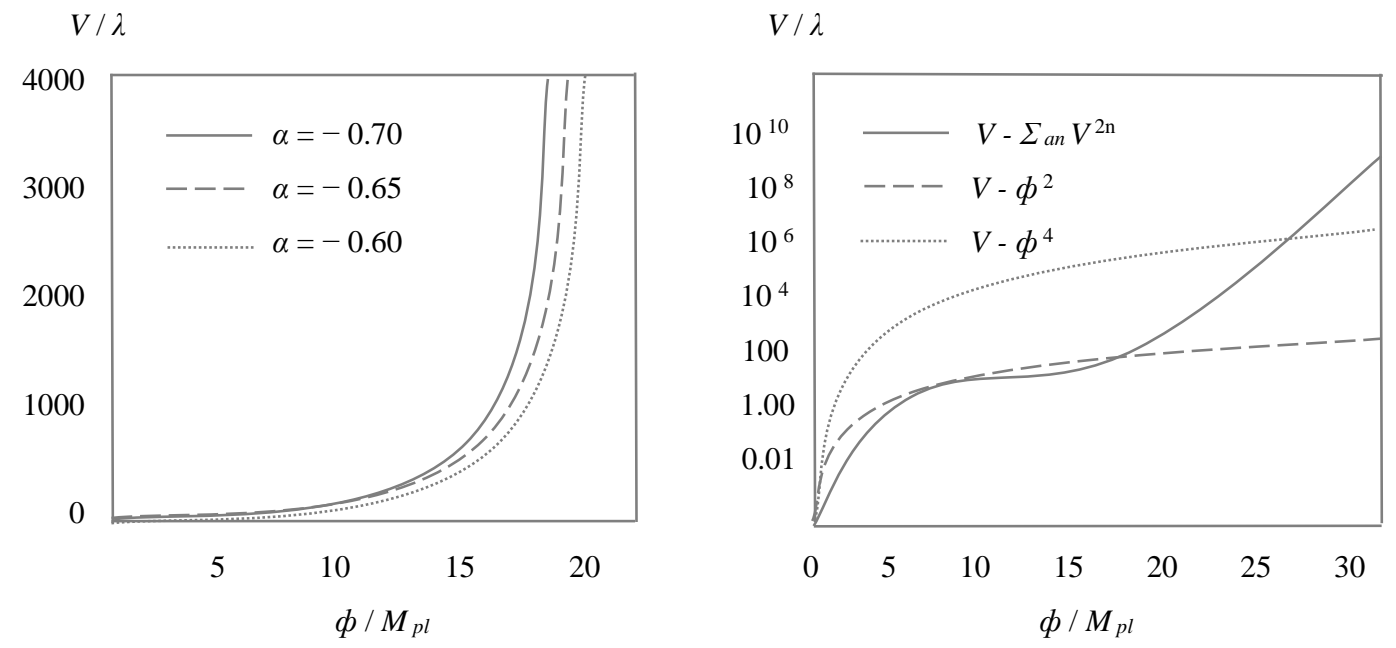

The value of parameter $\alpha$ is shown in the figure, and the value of $\beta$ is in its best fitting value

Fig. 3 left: potential energy $V$ in formula (1); right: compared $V \sim \phi^{2}, \phi^{4}$ with other forms of potential energy

First, we should distinguish two kinds of vacuum: cosmological vacuum and quantum mechanical vacuum; the former is a pseudovacuum with extremely high energy density, and the latter is a real vacuum with the lowest energy density.

Monopole can be formed in the process of $S U$ (5) symmetrical rupture. However, Gus's in-depth research shows that for elementary particles, the weight of a monopole is extraordinarily large, and its mass-energy is equivalent to $10{ }^{16} \mathrm{GeV}$. Therefore, the weight of a monopole is equivalent to 10,000 trillion $\left(10^{10}\right.$ ) protons, or to a bacterium, a small organism, in the universe, each proton corresponds to about one billion photons.

In the original model of the inflationary universe, the universe was created by pseudo-vacuum energy, and the Big Bang originated from pseudo-vacuum. In Einstein's theory, it is the density of matter and energy in the universe that determines the dynamics of space and time. Pseudo-vacuum 
energy is real energy, which may affect the universe and destroy the Big Bang, while the over-cooled universe is a universe that nurtures energy. Andrei Linde, Andreas Albrecht and Paul Steinhardt respectively found a solution to the problem of foam collision ${ }^{[5][50][55]}$. In this "slow-roll inflation" model, the mechanism of decay from the pseudo-vacuum state is no longer a quantum tunneling effect, but a scalar field rolling down from the potential peak. If the scalar field rolls down the potential energy peak at a much slower rate than the expansion of the universe, the inflation will occur. Once the potential peaks become steeper, the inflation will end and the reheating process will begin.

As shown in Fig. 4 (Li Zongcheng, 2019), each high-intensity quantum gravi-source forms a rip-rebound center for the Big Bang or explosion of the universe at the microscopic level. Here the dark part in the figure represents a larger density, while the light part represents a smaller density.

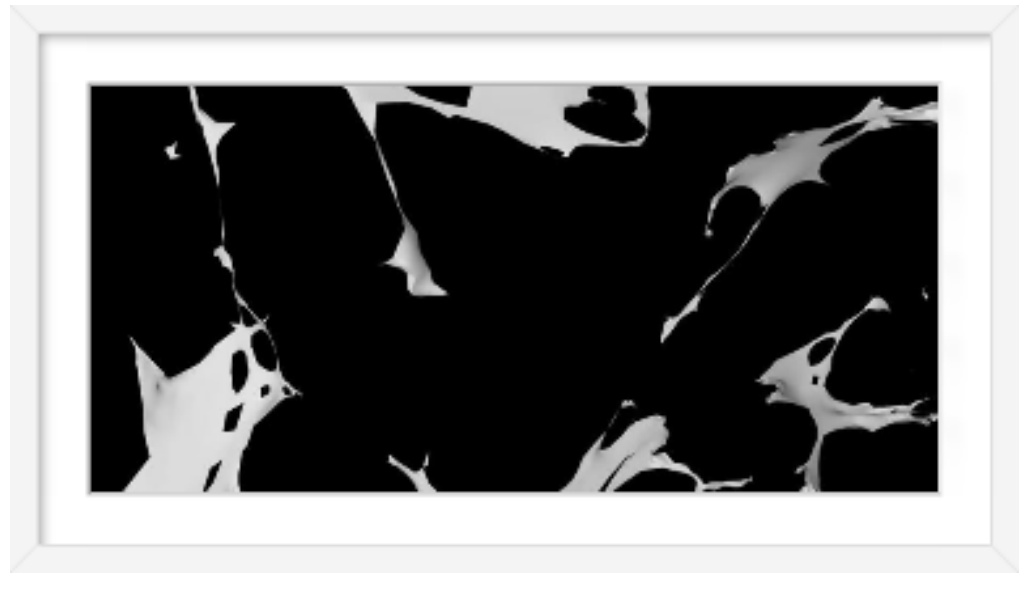

Fig. 4 Each high-intensity quantum gravi-source forms a rip-rebound center for the Big Bang or explosion of the universe at the microscopic level

As shown in Fig. 5 (Li Zongcheng, 2019), each initial high-intensity gravi-source forms a rip-rebound center for the Big Bang or explosion of the universe in macro-scale. Here the dark part of the figure shows a higher density, while the light part shows a smaller density.

Higgs energy, dark energy and gravitational energy also play an important role. The former two are mainly positive energy, and the latter are mainly negative energy ${ }^{[5][50][55]}$. From Planck time $\left(10^{-44}\right.$ sec) to the beginning of the inflation $\left(10^{-36} \mathrm{sec}\right.$ or $\left.10^{16} \mathrm{GeV}\right)$, it is a very important time. The end of the inflation is related to the cohesion of the Higgs field, which produces mass. In the universe, there are quantum random fluctuations. The scale of fluctuations is nearly equal. The matter and radiation of the universe are formed in this general fluctuation, and eventually galaxies and clusters are formed.

In existing cosmological estimations, the Big Bang of the Universe occurred 13.7 billion years ago $(t=0)$. In the $10^{-44} \mathrm{sec}$ of the creation of the universe, the intensity of the Big Bang just meets the requirement of breaking through four dimensions of space-time. In the subsequent expansion of the universe, the Higgs particle (Higgs boson) deviates from the center of symmetry and forms the lowest energy state, resulting in a symmetry break (phase transition of Higgs field). The excessive cooling of Higgs field leads to a pseudo-vacuum state, which is equivalent to igniting an atomic bomb $b^{[5][50][55]}$ during the explosion of a hand grenade. 
With regard to the super-inflating universe in the big impetus, we can put forward the following basic ideas here: In the very early universe, there was not only an accelerated expansion stage $(\ddot{R}>0)$, with an exponential growth $R \propto e^{H t}$ of the scale factor of the universe, in which $H$ was the Hubble constant; but also a big impetus stage from super-synergy (represented by the emergence of big rip-rebound clusters of quantum chaos-reticulate distribution), in which firstly super suppression is imposed, then super acceleration is imposed, finally super deceleration is imposed. For such a strong rip-rebound-inflation under a big impetus, we need to introduce a ultra-synergy scalar field $\phi_{B S}$, which satisfies the minimum coupling conditions with repulsion and gravity.

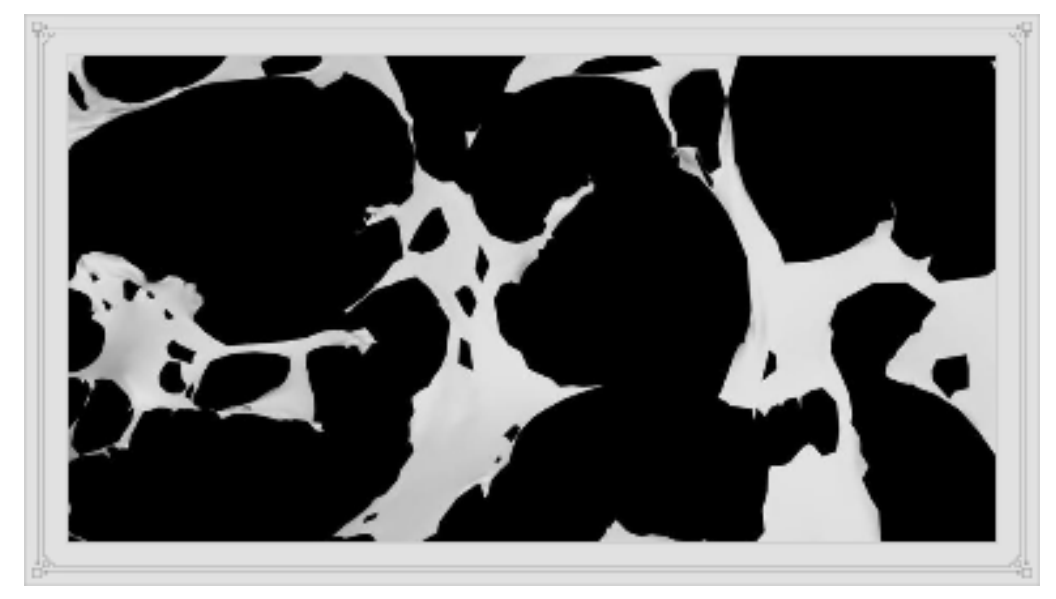

Fig. 5 Each initial high-intensity gravi-source forms a rip-rebound center for the Big Bang or explosion of the universe in macro-scale

The basic functions of ultra- synergy scalar fields can be expressed as follows:

$$
F_{u-s y n}=N^{b}\left[\left(1+\lambda_{d-e n}\right) \frac{\eta}{r^{s}}-\left(1+\lambda_{d-\text { matt }}\right) \frac{\mu}{r^{w}}\right], \quad(s>w),
$$

here $N$ is the total number of gravi-sources, $b$ is the super-inflation index, $r$ is the radius of gravitational action, $\eta, \mu, s, w$ are the theoretical values greater than zero, $\lambda_{d-e n}$ is the enhancement coefficient of dark energy, $\lambda_{d \text {-matt }}$ is the enhancement coefficient of dark matter, and the first term of the full expansion represents the effect of the repulsion of the universe as a whole on the $N^{b}$ gravi-sources, the second term represents the effect of primitive quantum gravity on these $N^{b}$ gravi-sources.

As shown in Figure 6 (Li Zongcheng, 2019), the big impetus mechanism of the inflationary universe in the big rip-rebound cluster of quantum chaos-reticulate distribution is discussed.

As shown in Fig. 4, Fig. 5 and Fig. 7 (Li Zongcheng, 2019), more and more gravi-sources and gauge field sources form a traction on the repulsion of the universe.

The ultra-synergy potential energy is expressed as follows:

$$
E_{u-s y n}=-\int_{\infty}^{r} F_{u-s y n} d r=N^{b}\left[\left(1+\lambda_{D E}\right) \frac{\eta^{\prime}}{r^{s-1}}-\left(1+\lambda_{D M}\right) \frac{\mu^{\prime}}{r^{w-1}}\right]
$$


here $\eta^{\prime}=\frac{\eta}{s-1}, \quad \mu^{\prime}=\frac{\mu}{w-1}$.

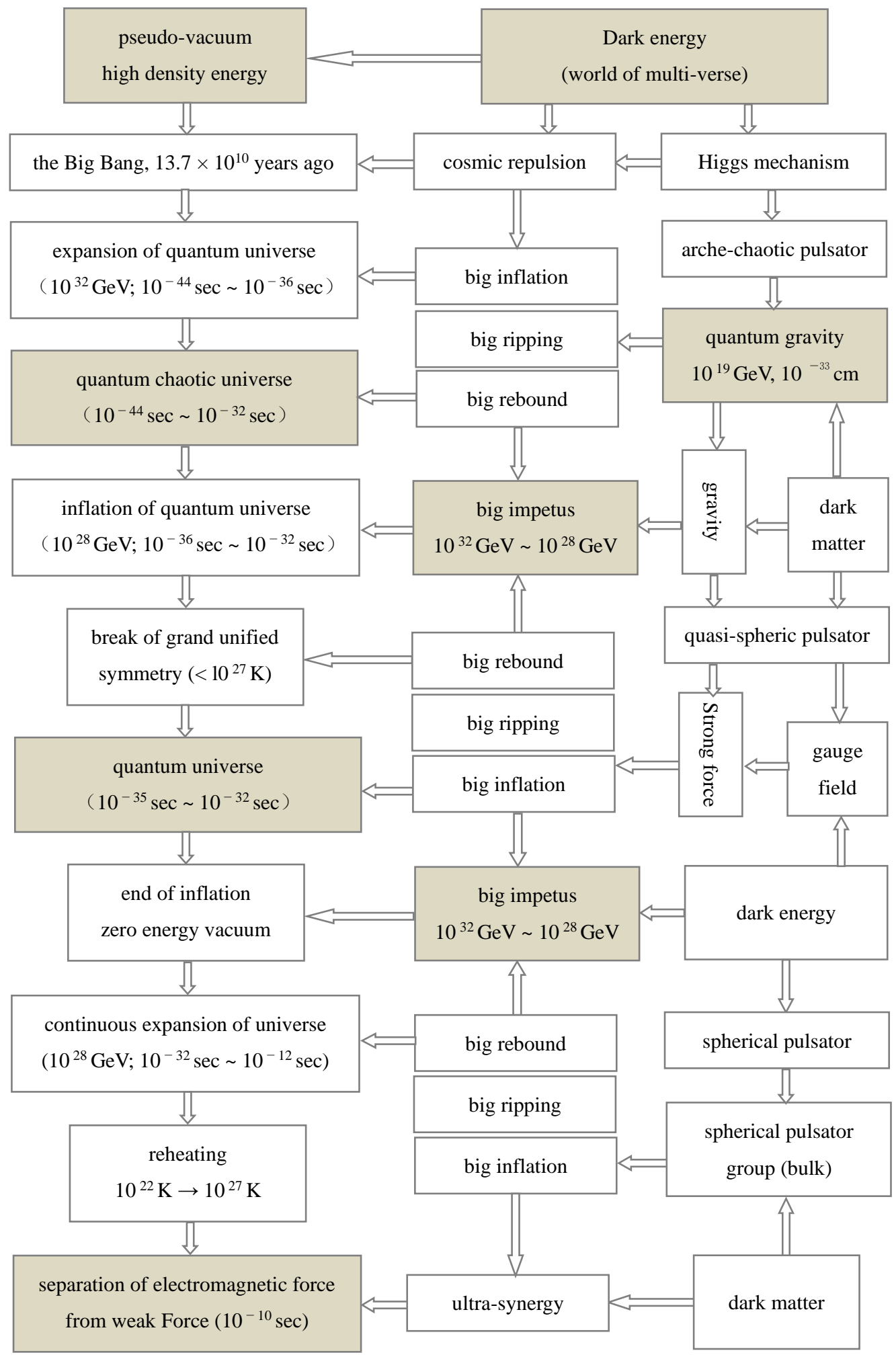

Fig. 6 The big impetus mechanism with big rip-rebound Cluster 


\section{of Quantum Chaotic-reticulate Distribution}

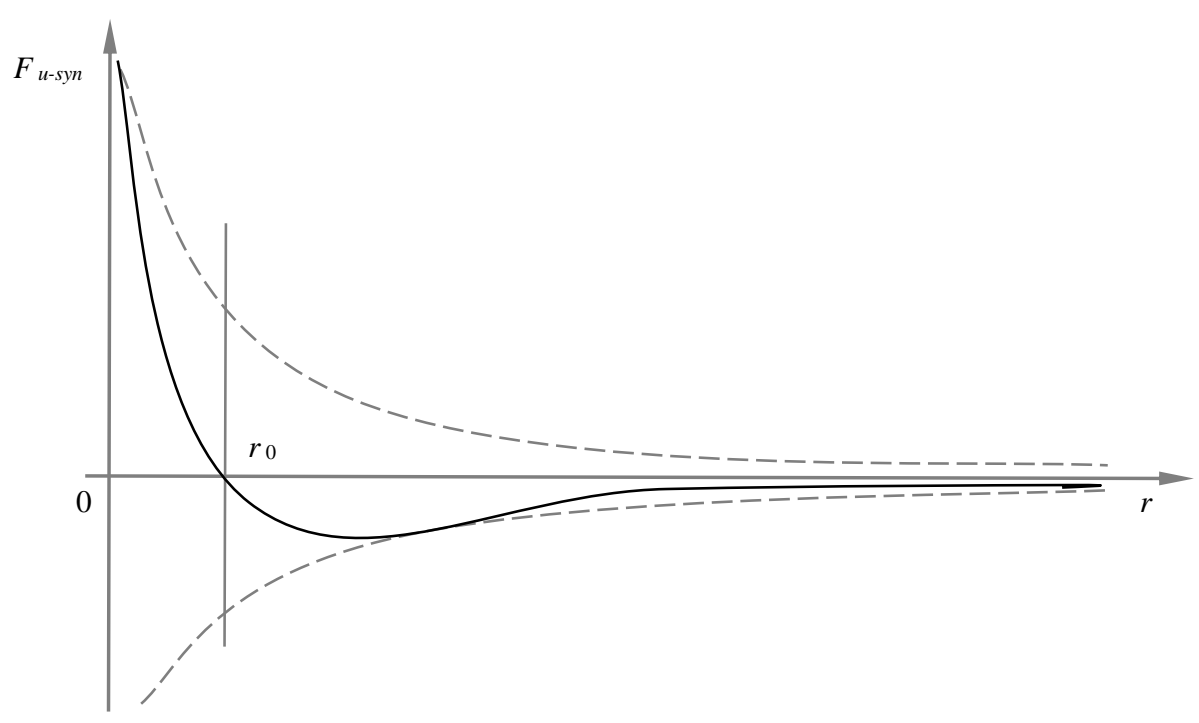

Fig. 7 Ultra-synergy of quantum universe with whole repulsion and inwards-pull

The energy density and pressure of the ultra-synergy scalar field can be expressed as follows:

$$
\rho_{\text {ultra-syn }}=\frac{1}{2} \dot{\phi}_{B S}^{2}+(1-A) V\left(\phi_{B S}\right), \quad p_{\text {ultra-syn }}=\frac{1}{2} \dot{\phi}_{B S}^{2}-(1-A) V\left(\phi_{B S}\right),
$$

here $V\left(\phi_{B S}\right)$ is the potential energy of ultra-synergy field, $A=-\ddot{R} / R H^{2}, A$ is a binary quantity:

$$
A=\left\{\begin{array}{lll}
-\ddot{R} / R H^{2}>0 & A & \text { is decelerating factor } \\
-\ddot{R} / R H^{2}<0 & A & \text { is accelerating factor }
\end{array}\right.
$$

Expression ( 63 ) can be written in the following form:

$$
\begin{array}{r}
\rho_{\text {ultra-syn }}=N^{b}\left\{\left(1+\lambda_{d-e n}\right)\left[\frac{1}{2} \dot{\phi}_{\text {repl }}^{2}+\left(1-A_{\text {acce }}\right) V\left(\phi_{\text {repl }}\right)\right]\right. \\
\left.-\left(1+\lambda_{d-\text { matt }}\right)\left[\frac{1}{2} \dot{\phi}_{\text {grav }}^{2}+\left(1-A_{\text {dece }}\right) V\left(\phi_{\text {grav }}\right)\right]\right\} \\
p_{\text {ultra-syn }}=N^{b}\left\{\left(1+\lambda_{d-e n}\right)\left[\frac{1}{2} \dot{\phi}_{\text {repl }}^{2}-\left(1-A_{\text {acce }}\right) V\left(\phi_{\text {repl }}\right)\right]\right. \\
\left.-\left(1+\lambda_{d-\text { matt }}\right)\left[\frac{1}{2} \dot{\phi}_{\text {grav }}^{2}-\left(1-A_{\text {dece }}\right) V\left(\phi_{\text {grav }}\right)\right]\right\}
\end{array}
$$

here $N$ is the total number of gravi-sources, $b$ is the super-inflation index, $\phi_{\text {repl }}$ is the scalar field under the repulsion of the universe, $\phi_{\text {grav }}$ is the scalar field under the quantum gravity, $A_{\text {acce }}$ is accelerating factor, $A_{\text {dece }}$ is decelerating factor, $\lambda_{d \text {-en }}$ is the enhancement coefficient of dark energy, $\lambda_{d \text {-matt }}$ is the 
enhancement coefficient of dark matter, and the first term of the full expansion ( $65 a$ ) represents the energy density under the repulsion of the whole universe to $N^{b}$ gravi-sources, and the second term represents the energy density under the gravitation of the arche-quantum to $N^{b}$ gravi-sources, the first term of the full expansion ( $65 b$ ) represents the pressure under the repulsion of the whole universe to $N^{b}$ gravi-sources, and the second term represents the pressure under the repulsion of the whole universe to $N^{b}$ gravi-sources, and the second term represents under the gravitation of the arche-quantum to $N^{b}$ gravi-sources.

The extended form of Klein-Gorden equation for ultra-synergy scalar field should be

$$
\ddot{\phi}_{B S}+3 H \dot{\phi}_{B S}+(1-A) \frac{d V}{d \phi_{B S}}=0
$$

Assuming that the ultra-synergy scalar field is dominant in all components during the rip-reboundinflation stage of the big impetus, the extended form of Freeman equation should be

$$
H^{2}=\frac{8 \pi G}{3}\left[\frac{1}{2} \dot{\phi}_{B S}^{2}+(1-A) V\left(\phi_{B S}\right)\right]
$$

Expression ( 67 ) can be further written in the following form:

$$
\begin{aligned}
H_{u-s y n}^{2}=N^{b}\left\{\left(1+\lambda_{d-e n}\right)\right. & \frac{8 \pi G}{3}\left[\frac{1}{2} \dot{\phi}_{\text {repl }}^{2}+\left(1-A_{\text {acce }}\right) V\left(\phi_{\text {repl }}\right)\right] \\
& \left.-\left(1+\lambda_{d-\text { matt }}\right) \frac{8 \pi G}{3}\left[\frac{1}{2} \dot{\phi}_{\text {grav }}^{2}+\left(1-A_{\text {dece }}\right) V\left(\phi_{\text {grav }}\right)\right]\right\}
\end{aligned}
$$

(68)

The model of the super-inflating universe with the big impetus sets up a effective potential $V(\varphi)$ of the big impetus similar to Figure 8, which comes from the interaction among the Higgs field and the initial gravitational field (the gravity only just separated from quantum gravity is very strong) as well as the grand unified field of super-symmetry. When $T=0$, the total minimum value of the big impetuseffective potential is called the real vacuum, and $\varphi=\varphi_{\text {true }}$. the current scalar fields in the universe are experiencing small fluctuations to real vacuum values, while the value of $V\left(\varphi_{\text {true }}\right)$ represents the energy density of vacuum, and the relation related to the cosmic constant $\Lambda$ is $\Lambda=8 \pi G \cdot V\left(\varphi_{\text {true }}\right)$.

The effective potential of the big impetus at $T=0$ has a second local minimum ${ }^{[5][50][55]}$, where the value of $\varphi$ is denoted as $\varphi_{\text {false }}$, and the state of the expected value $\langle\varphi\rangle=\varphi_{\text {false }}$ of the big impetuseffective field is called a pseudo-vacuum, and the energy density is denoted as $\rho_{f}$. Furthermore, suppose there is a critical temperature $T_{c}$, above which the value of the big impetus-effective potential at finite temperature near the pseudo-vacuum is less than that near the real vacuum. As shown in Figure 8 (Li Zongcheng, 2019), $T_{c}$ is the critical temperature of the first-order phase transition.

Le $T_{B S, \mu \nu}$ be a covariant conserved tensor consisting of the big impetus-effective field containing Higgs field, the metric and the primary and secondary derivatives of this field. We can derive the energy-momentum tensor $T_{B S, \mu \nu}$ of the big impetus-effective field in a pseudo-vacuum. Because the big impetus-effective field is a constant in pseudo-vacuum, the only tensor for $T_{B S, \mu v}$ is $g_{B S, \mu v}$, and 


$$
G_{B S, \mu v} \equiv R_{\mu v}-\frac{1}{2} R g_{B S, \mu v}
$$

So, $T_{B S, \mu \nu}$ has the following form: $T_{B S, \mu v}=A g_{B S, \mu v}+B G_{B S, \mu v}$, here $A$ and $B$ are constant. The equation of ultra-synergy field including Einstein field is

$$
G_{B S, \mu v}=-8 \pi G T_{B S, \mu v}
$$

Therefore, the term $B G_{B S, \mu \nu}$ can be absorbed into the $T_{B S, \mu \nu}$ on the left side of the equation by redefining the gravitational constant $G$. Reusing the energy-momentum tensor form of ideal fluid

$$
T_{B S, \mu v}=p g_{B S, \mu v}+(\rho+p) u_{\mu} u_{v}
$$

we determine constant $\mathrm{A}$ to be just the negative value of pseudo-vacuum energy density, so $T_{\mu \nu}=-\rho_{f} \cdot g_{\mu \nu}$. This formula means the pressure $p=-\rho_{f}$ of the pseudo-vacuum.

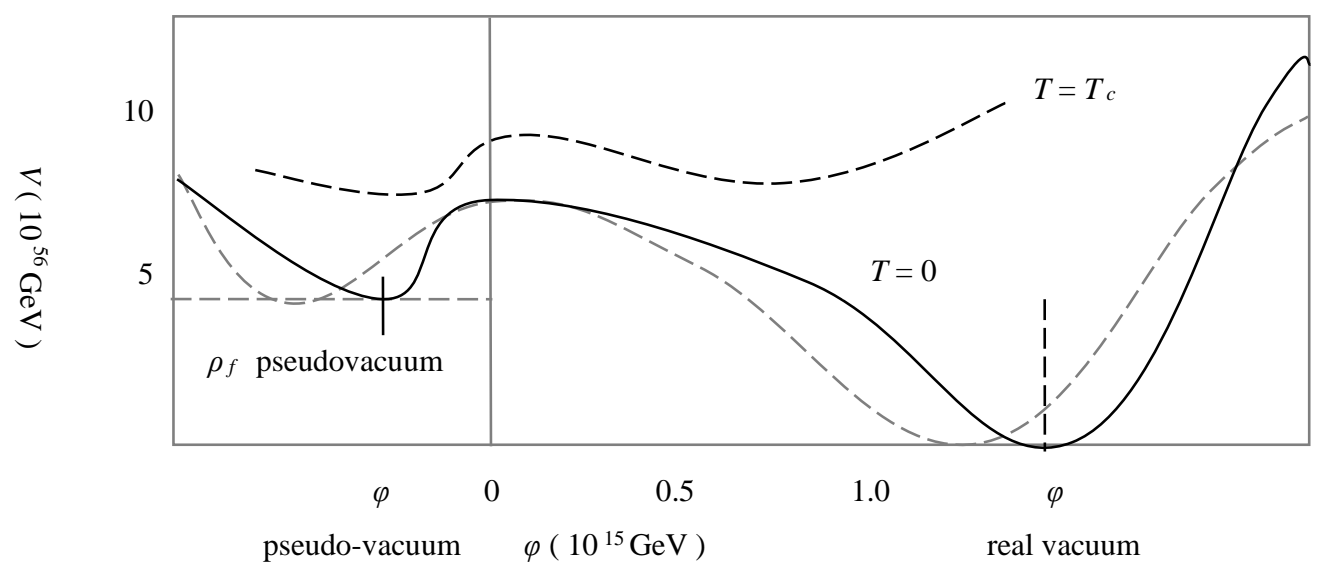

Fig. 8 Curve of Big Impetus-Effective Potential Function Adjusted to the New and Old Inflationary Cosmological Models

In the new models set up in this series, we should take into account a very important but often neglected situation: when gravity, strong force and electro-weak force are separated from super-gravity or quantum gravity respectively, they are accompanied by more and more new-born field sources and the expansion of the universe as a whole to form big ripping clusters. This large tear cluster will temporarily hinder the whole expansion of the universe at the beginning, but it will become a big impetus force for the whole expansion of the universe immediately near the end of the big ripping.

For this reason, we set the scalar functional:

$$
I=I_{B}+I_{S}+I_{B S}=\int\left(L_{B}+L_{S}+L_{B S}\right) \sqrt{-g} d^{4} x
$$

here

$I_{B}$ and $L_{B}$ represent the action and its Lagrangian function of the whole Big Bang (outwards-push) of the universe, respectively.

$I_{S}$ and $L_{S}$ represent the action and its Lagrangian functions of gravity (inwards-pull) respectively. 
$I_{B S}$ and $L_{B S}$ represent the interaction between the whole Big Bang and gravity and their Lagrangian functions, respectively.

The expression of $I_{S}$ and $L_{S}$ is taken as

$$
L_{S}=R, \quad L_{B}=-2 k L_{B} .
$$

here $k$ is Einstein gravitational constant, $k=8 \pi G / c^{4}$.

Here the variational principle can be expressed as: $\delta I=\delta\left(I_{B}+I_{S}+I_{B S}\right)=0$.

As shown in Figure 9 (Li Zongcheng, 2019), in the initial stage of the Big Bang $\left(0 \sim 10^{-38} \mathrm{sec}\right)$, more and more quantum gravitational sources and the Big Bang of the universe formed big ripping, which should temporarily hindered the initial Big Bang of the universe. However, before and after the end of the big ripping, it would instantly become big impetus to the whole Big Bang of the universe. When gravity separates from quantum gravity (around $10^{-40} \mathrm{sec}$ ), the universe undergoes phase transition, more and more gravi-sources and the whole expansion of the universe form a big ripping in the macro scope. However, before and after the end of the big ripping, it would instantly become big impetus to the whole expansion of the universe. When strong force separates from the grand unified force of super-symmetry (around $10^{-35} \mathrm{sec}$ ), the universe undergoes phase transition, more and more strong force sources and the whole expansion of the universe form a big ripping in the micro scope. However, before and after the end of the big ripping, it would instantly become big impetus to the whole inflation of the universe. When electro-weak force separates from the gauge field (around $10^{-10}$ sec), the universe undergoes phase transition, more and more electro-weak force sources and the whole expansion of the universe form a big ripping in the micro scope. However, before and after the end of the big ripping, it would instantly become big impetus to the whole expansion of the universe.

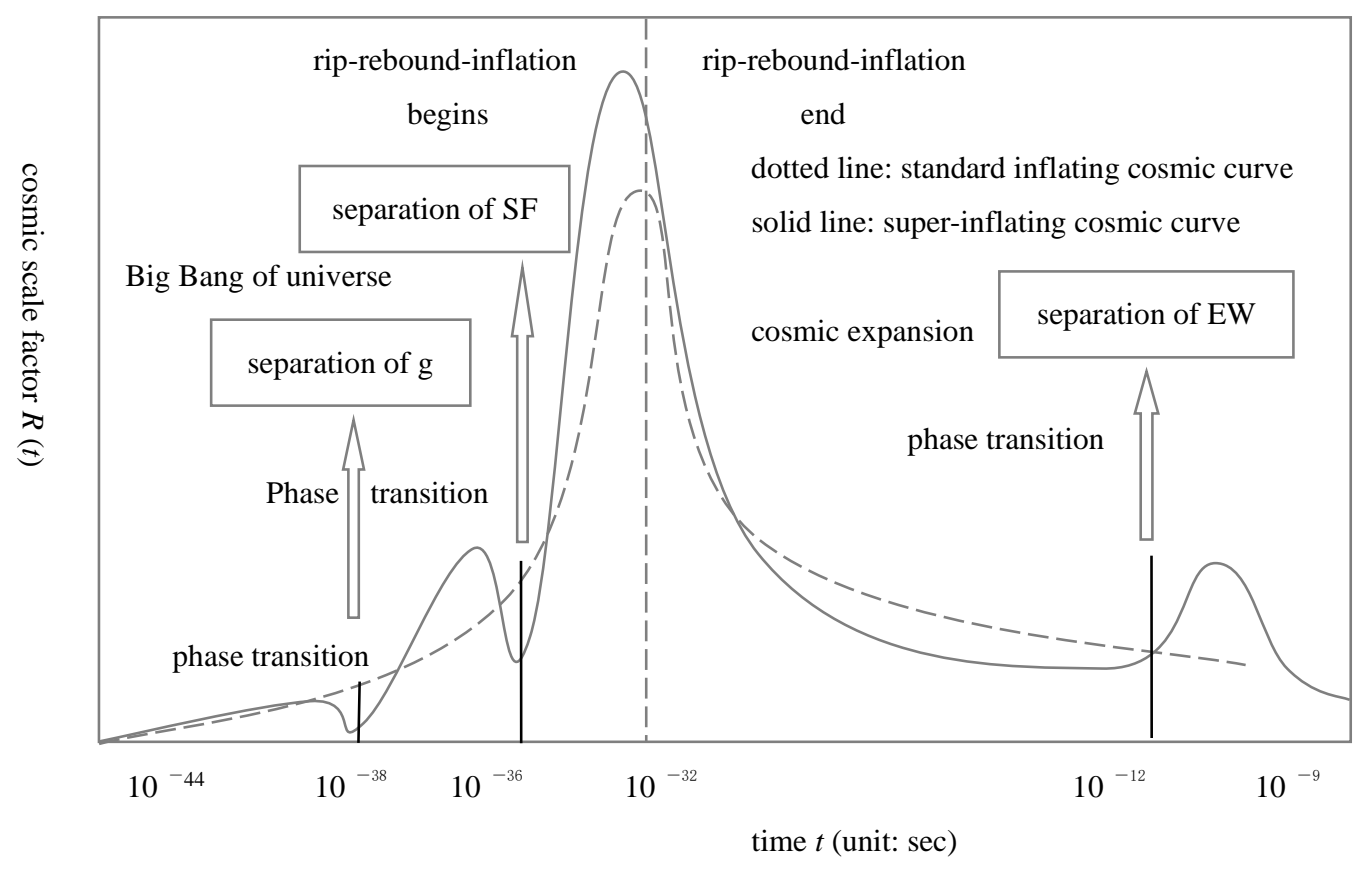

Figure 9 Cosmic Phase Transition, Separation of Forces and Cosmic Rip-Rebound-Inflation 
For those regions which are still in the pseudo-vacuum state after supercooling, we give the Einstein field equation as follows:

$$
\left(\frac{\dot{R}}{R}\right)^{2}=\frac{8 \pi}{3} G p_{f}
$$

Its solution is $R(t) \propto e^{\chi t}$, here $\chi=\sqrt{\frac{8 \pi G}{3} \rho_{f}} \approx 10^{10} \mathrm{GeV} \approx 10^{34} \mathrm{sec}^{-1}$.

The main result of the huge energy density $\rho_{f}$ of pseudo-vacuum is the exponential growth of scaling factor $R(t)$, and the time constant is $\chi^{-1}$. The exponential of the big impetus is described by the flat Robertson-Walker metric, and the space with exponential growth scaling factor is de Sitter space.

For ultra-synergy dynamics under the big impetus, we should consider not only the second-order Einstein field equation.

$$
\ddot{R}=-\frac{4 \pi}{3} G(\rho+3 p) R
$$

This equation means that the deceleration $-\ddot{R}(t)$ of the cosmic scaling factor is proportional to $(\rho+3 \mathrm{p})$. During the rip-rebound-inflation with the big impetus, $p=-\rho=-\rho_{f}$, so the negative contribution of pressure exceeds the positive term of energy density. On the contrary, the macroscopic ripping between the cosmic outwards-push induced by Higgs field and gravity, and the microscopic ripping between the cosmic outwards-push induced by Higgs field and the strong force subsequently just separated from the grand unified gauge field induced, have a synthetical effect (i.e. big impetus) of enhancing expansion, so $\dot{R}$ increases monotonously with time.

In other words, for the cosmic inflation, the ultra-synergy between the cosmic outwards-push induced by the Higgs field and the quantum gravity as well as initial gravity only just separated from high-dimensional supergravity, and between the cosmic outwards-push induced by the Higgs field and the initial strong force only just separated from gauge field, form a big impetus that first blocks, then turns into super-acceleration, and then into super-deceleration.

It is noticed that the dynamics of the big impetus-effective scalar field is determined by the effective potential function, and the perturbation behavior at $\varphi=0$ is determined by the effective potential near $\varphi=0$. Assuming that the big impetus-effective potential is very flat near $\varphi=0$, for the sake of clarity, assuming that at $\varphi=0$, there should be $\frac{\partial V_{B S}}{\partial \varphi}=0,\left|\frac{\partial^{2} V_{B S}}{\partial \varphi^{2}}\right| \leq \chi^{2},\left|\frac{\partial^{3} V_{B S}}{\partial \chi^{3}}\right| \leq \chi$.

When the grand unified symmetry is broken, the energy is $10{ }^{16} \mathrm{GeV}$, the temperature is $10^{27} \mathrm{~K}$, the phase transitions occur and the bubbles increase. At the same time, the quantum fluctuations (gravitons bearing gravity) of micro-gravitational waves and space-time increase extremely. Later, a freeze occurs instantaneously, triggering a polarization eddy (the new discovery of microwave background radiation is considered a triumph of the inflationary universe theory). The freezing of the Higgs field is slowed down by the big impetus between the cosmic expansion force and the basic physical force.

Random quantum fluctuations in the energy field of the universe can increase the vacuum energy 
density somewhere and make that part of the universe erupt violently into a state of inflation. In the model of the super-inflating universe with the big impetus, the unified inflation of the universe as a whole and the numerous local inflations of the universe emerge together.

\section{Model (II) of Super-Inflating Universe with Big Impetus}

Under the ultra-synergistic effect with the big rip-rebound cluster, although the inflation of the universe is accelerated exponentially, for example, the expansion of the universe exceeds the proportion of $e^{60}\left(\approx 10^{26}\right)$, it always goes through instantaneous blockades and then swiftly goes into super-inflation, in every process of the phase transition of the universe and the separation of basic physical action. In this process, the diameter of the universe increases multiply, the volume of the universe increases exponentially, and the pseudo-vacuum energy of the universe also increases exponentially. When the universe inflates to $10^{-33} \mathrm{sec}$, the volume of the universe changes to $10^{28} \mathrm{ft}$ $\left(0.3048 \times 10^{25} \mathrm{~km}\right)$. At the end of the inflation, the diameter of the universe can reach dozens of light-years. As a result, the universe escapes faster, making it flat, homogeneous and isotropic on the largest scale.

In the stage of cosmic inflation, there are quantum fluctuations everywhere, and there are subtle temperature differences in various regions of the universe. With the expansion of the space caused by the inflation, the number density of large-mass singular particles (for example, magnetic monopoles predicted by the standard model of particle physics) decreases rapidly ${ }^{[6]-[8]}$. Today, there is no monopole in the visible universe, so the "hairless effect" (theorem) of cosmic inflation has appeared, which is similar to the black hole hairless theorem.

The thermal effect is important in the initial fluctuations, but the temperature is quickly neglected as the rip-rebound-inflation continues. So there is $\square \varphi=-\partial V_{B S} / \partial \varphi$, which in the de Sitter metric becomes

$$
\ddot{\varphi}+3 \chi \dot{\varphi}=-\frac{\partial V_{B S}}{\partial \varphi}
$$

If we take $\varphi$ as a position coordinate, then this equation describes the motion of particles in the big impetus-effective field $V_{B S}(\varphi)$ with damping force $3 \chi \dot{\varphi}$. this damping term reflects the expansion of the universe. It is this damping term that makes the model of super-inflating universe with big impetus possible. Under the action of the big impetus, the scalar field must decline slowly enough along the potential $V_{B S}(\varphi)$ to make the coherent region possible to experience a prolonged explosion.

When $\varphi \approx 0$, the energy density is very close to that of $\rho_{f}$, so the exponential expansion is maintained during the slow rolling process. The typical scale of the coherent region will be stretched to $Z \chi^{-1}$, where $Z=e^{\chi t}$, and $\Delta t$ represents the time of exponential expansion.

However, when the effective scalar field in the big impetus reaches the steep part of the potential $V_{B S}(\varphi)$, it drops rapidly to the minimum, and then the minimum oscillations occur, which will be damped by the coupling terms of the $\varphi$ field and other fields in the motion course of the big impetus. Similar to the original inflation model, the temperature after reheating can be calculated by energy 
conservation, and its typical value is still $T_{B S, r e h}=\frac{1}{3} T_{B S, c}$.

It is generally believed that within the framework of classical gravity theory, problems such as singularity of the Big Bang, dark energy, origin and formation of the large-scale structure of the universe can not be solved ${ }^{[6]-[8]}$. For further calculation, we make Fourier expansion for the big impetus-effective scalar field $\varphi$ :

$$
\varphi(x, t)=\frac{1}{(2 \pi)^{3 / 2}} \int d^{3} k\left\{d(k) e^{i k x} \psi_{B S}(k, t)+d^{+}(k) e^{-i k x} \psi_{B S}^{*}(k, t)\right\}
$$

The ultra-synergy modular function $\psi_{B S}(k, t)$ satisfies the following equations of motion:

$$
\ddot{\psi}_{B S}+3 \chi \dot{\psi}_{B S}=\left[\mu^{2}-e^{-2 \chi t}\left(k^{2}+\gamma^{2}\right)\right] \psi_{B S}
$$

here $\gamma=\frac{1}{4} a \bar{T}_{B S}^{2}$. When $t \rightarrow-\infty$, the equation satisfied by each $\psi_{B S}(k, t)$ approximates to the arche-oscillating equation of the harmonic oscillator, whose frequency is $\omega(t)=e^{-\chi t} \sqrt{k^{2}+\gamma^{2}}$.

When the universe expands exponentially under the big impetus action of super-synergy, the frequency drift rate of the harmonic oscillator has the magnitude of $\chi$. However, when $t \rightarrow-\infty$, the frequency of the harmonic oscillator is $\omega \rightarrow \infty$, so there is $\omega>>\chi$ in the early stage.

Equation ( 76 ) has two linearly independent solutions, each of which is the product of an exponential function and a Bessel function. We can choose the appropriate linear combination to make it consistent with the standard treatment of free field theory in Minkowski space. So we chose

$$
\psi_{B S}(k, t)=\frac{1}{2} \sqrt{\frac{\pi}{\chi}} e^{-\frac{3}{2} \chi t} H_{B S, P}{ }^{(1)}(Z)
$$

here $H_{B S, P}^{(1)}(Z)$ is the extended Hankel function, and

$$
P=\sqrt{\frac{9}{4}+\frac{\mu^{2}}{\chi^{2}}}, \quad Z=\frac{\sqrt{k^{2}+\gamma^{2}}}{\chi} e^{-\chi t}
$$

The linear combination of ( 78 ) is chosen because when $t$ gradually approaches zero, it has the following form:

$$
\psi_{B S}(k, t) \sim e^{i \theta(t)} e^{-3 \chi t / 2} \frac{e^{-i \omega t}}{\sqrt{2 \omega(t)}}
$$

here $e^{i \theta(t)}$ is a slowly varying phase factor. There is no factor $e^{-3 \chi^{t / 2}}$ in Minkowski space, which is a slow drift in normalization conventions. The use of regular commutation relations for ( 78 ) and ( 75 ) formulas proves that:

$$
\left[d(k), d^{+}\left(k^{\prime}\right)\right]=\delta^{3}\left(k^{\prime}-k\right)
$$

After giving the behavior ( 79 ) and the commutation relation ( 80 ) of the ultra-synergetic modular function, we will see that the early operators $d^{+}(k)$ and $d(k)$ can be interpreted as the generation and 
annihilation operators of arche-pulsators as Minkowski-like particles.

To obtain a measurable ultra-synergistic operator, we have to "fuzzify" the effective quantum field with the big impetus in a finite volume, and define a "fuzzifying" ultra-synergistic field

$$
\hat{\varphi}_{l}(x, t)=\frac{1}{(2 \pi)^{3 / 2} l^{3}} \int d^{3} y e^{-(x-y)^{2} / 2 l^{2}} \varphi(y, t)
$$

here $l$ is the length of "fuzzification" described by co-moving coordinates.

Considering the one-loop correction of ultra-synergistic gauge interaction, the big impetuseffective potential at zero temperature is as follows

$$
V_{B S}(\varphi)=\frac{25}{16} \alpha^{2}\left\{\varphi^{4} \ln \left(\varphi^{2} / \sigma^{2}\right)+\frac{1}{2}\left(\sigma^{4}-\varphi^{4}\right)\right\}
$$

here $\alpha=g^{2} / 4 \pi \approx 1 / 45, g$ is the gauge coupling constant, $\sigma \approx 1.2 \times 10^{15} \mathrm{GeV}$. The form of this potential is shown in Figure 8 above. The minimum value of $V_{B S}(\varphi)$ is at $\varphi=\sigma$, corresponding to the actual vacuum, and $\varphi=0$ is an almost unstable equilibrium point. At zero temperature, the configuration of $\varphi=0$ is called pseudo-vacuum.

The big impetus-effective potential at finite temperature is

$$
V_{B S}(\varphi, T)=V_{B S}(\varphi)+\frac{18 T}{\pi^{2}} \int_{0}^{\infty} d q \cdot q^{2} \ln \left[1-\exp \left(-\sqrt{q^{2}+\frac{5 \pi}{3} \alpha \varphi^{2} / T_{B S}}\right)\right]
$$

The form of $V_{B S}\left(\varphi, T_{B S}\right)$ is shown in Figure 10 (Li Zongcheng, 2019).

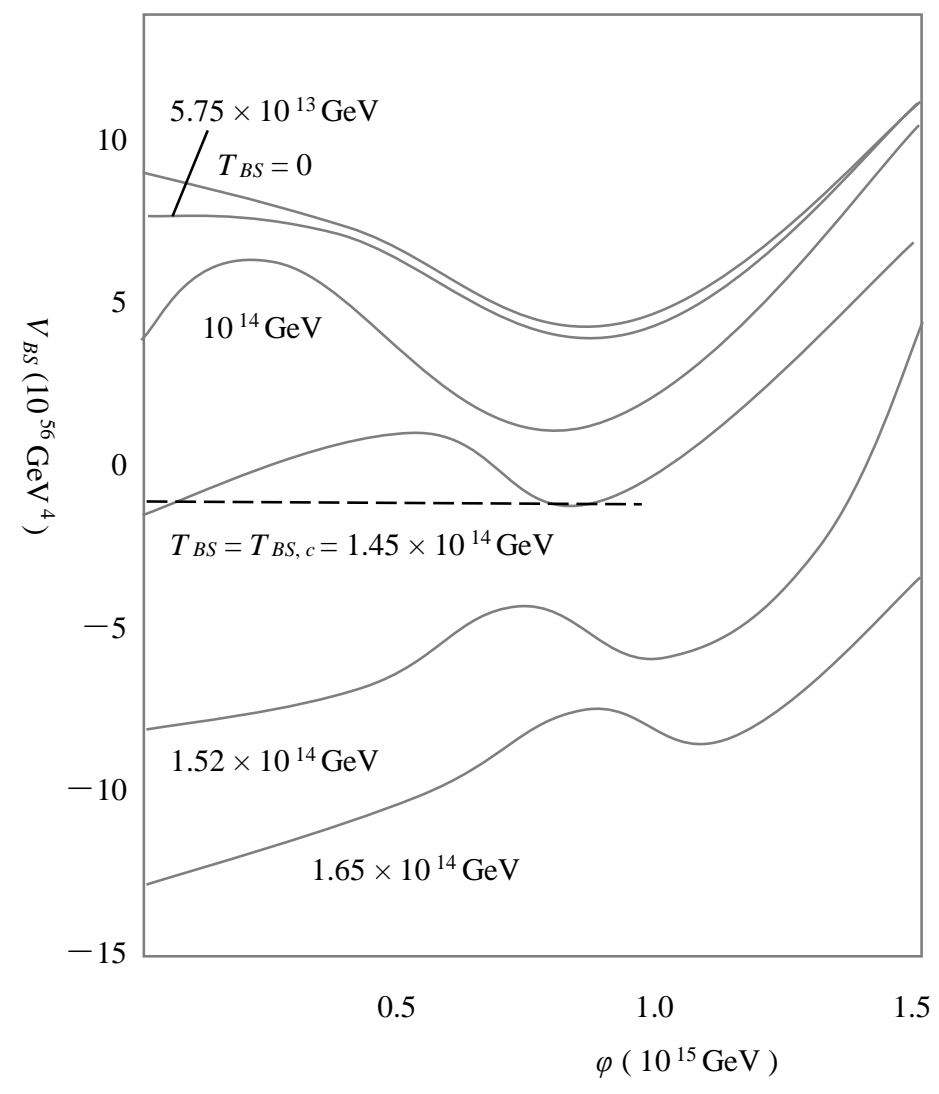




\section{Figure 10 Function Curve of Big Impetus-Effective Potential in Finite Temperature}

These ultra-synergistic curves undergo first-order phase transition at $T_{B S}=T_{B S, c}=1.25 \times 10^{14}$ $\mathrm{GeV}$. When $T_{B S}<T_{B S, c}$, c, the minimum value of the big impetus-effective potential is in the range of $\varphi=\sigma$; and when $T_{B S}>T_{B S, c}$, the minimum value of the big push effective potential is in the range of $\varphi$ $=0$. When $0<T_{B S}<T_{B S, c}$, there exists a metastable phase of $\varphi=0$, corresponding to the local minimum of the big impetus-effective potential.

With the inflation of the universe, the supercooled expansion occurs, the temperature drops rapidly from $10^{27} \mathrm{~K}$ to $10^{22} \mathrm{~K}$, the huge potential energy of the inflationary field decays, and some particles (including electromagnetic radiation particles) of standard model appears ${ }^{[6]-[8]}$. As a result, reheating or heating occurs, the temperature recovers from $10^{22} \mathrm{~K}$ to $10^{27} \mathrm{~K}$, which leads to the particles in relativity plasma (quark-gluon plasma), so that the universe entered a radiation-dominated period (in the form of quarks, electrons and neutrinos).

Referring to the calculation of the inflation universe theory and the new research in this series, we can infer that after the inflation, the scale of the universe should expand about $e^{70}$, the quantum fluctuation should expand rapidly, while the material-energy distribution should show irregularity, and the state of supercooling should appear. On a larger scale, there should be multiple universes with constant inflation and explosion.

Furthermore, by the method in literatures [97] and [98], we introduce non commutation into inflation cosmology, which can better explain the source of running spectrum index in a natural way and get the results consistent with the experiment.

Non commutation is studied by Moyal product. Therefore, in general curved space-time, we define the Moyal product:

$$
f_{1} * \cdots * f_{n}=\left(1+\frac{i}{2} \theta^{\mu \nu} \sum_{a<b} D_{\mu}^{a} D_{v}^{b}-\frac{1}{8} \theta^{\mu v} \theta^{\rho \sigma} \sum_{a<b, c<d} D_{\mu}^{a} D_{v}^{b} D_{\rho}^{c} D_{\sigma}^{d}\right) f_{1} \cdots f_{n},
$$

The lower order disturbance (accurate to $O\left(\theta^{2}\right)$ ) is:

$$
\left.\delta_{\theta} f_{1} * \cdots * f_{n}=-\frac{1}{8} \theta^{\mu v} \theta^{\rho \sigma} \sum_{a<b, c<d} D_{\mu}^{a} D_{v}^{b} D_{\rho}^{c} D_{\sigma}^{d}\right) f_{1} \cdots f_{n} .
$$

Set $\theta^{0 i}=0$ by unitary. Considering the non commutativity, we take $\theta^{12}=l_{p}^{2} / a^{2}$ in the co-moving coordinate.

The non commutative correction of kinetic energy $X=-\frac{1}{2} \partial_{\mu} \phi \partial^{\mu} \phi$ is calculated as follows:

$$
\delta_{\theta} X=\frac{1}{8}\left(\theta^{12}\right)^{2} g^{\mu \nu}\left(D_{1} D_{1} D_{\mu} \phi D_{2} D_{2} D_{\nu} \phi-D_{1} D_{2} D_{\mu} \phi D_{2} D_{1} D_{\nu} \phi\right)
$$

It is generally assumed that there is a inflation, and its non commutation correction is as follows: 


$$
\begin{aligned}
\delta_{\theta} V= & \frac{n(n-1)}{8}\left(\theta^{12}\right)^{2} \phi^{n-2}\left(D_{1} D_{1} \phi D_{2} D_{2} \phi-D_{1} D_{2} \phi D_{2} D_{1} \phi\right) \\
& -\frac{n(n-1)(n-2)}{24}\left(\theta^{12}\right)^{2} \phi^{n-3}\left(D_{1} D_{1} \phi D_{2} D_{2} \phi+D_{2} D_{2} \phi D_{1} D_{1} \phi\right. \\
& \left.-D_{1} D_{2} \phi D_{1} \phi D_{2} \phi-D_{2} D_{1} \phi D_{2} \phi D_{1} \phi\right) .
\end{aligned}
$$

Considering the lowest order modification of the correlation function $\theta^{2}$, and using $\delta_{\theta} X$ and $\delta_{\theta} Y$ to identify the correction of kinetic energy and potential energy respectively, the corrected action of the inflaton is as follows:

$$
S=\int d^{4} x \sqrt{-g}\left(P_{X} \delta_{\phi} X+P_{V} \delta_{\theta} V\right) .
$$

In the vicinity of background space-time, $P_{X}$ and $P_{Y}$ are transformed into terms of $\delta_{\theta} X$ and $\delta_{\theta} Y$ by Taylor expansion. At the same time, taking the leading order of slow roll parameter, we get:

$$
\begin{aligned}
\delta_{\theta} S_{2}= & \int d^{4} x \sqrt{h}\left(P_{X_{0}}\left(\delta_{\theta} X\right)_{2}+P_{X_{0} X_{0}}\left(\delta_{g} X\right)_{1}\left(\delta_{\theta} X\right)_{1}\right. \\
& \left.+P_{V_{0}}\left(\delta_{\theta} V 1\right)_{2}+P_{V_{0} X_{0}}\left(\delta_{g} X\right)_{1}\left(\delta_{\theta} V\right)_{1}\right)+P_{X_{0} \phi_{0}} \varphi\left(\delta_{\theta} X\right)_{1},
\end{aligned}
$$

where,

$$
\delta_{g} X=X-X_{0}, \quad\left(\delta_{g} X\right)_{1}=\dot{\phi}_{0} \dot{\varphi}, \quad\left(\delta_{g} X\right)_{2}=\frac{1}{2}\left(\dot{\varphi}^{2}-(\partial \varphi)^{2}\right)
$$

We can decompose the perturbation of potential $\delta_{\theta} X$ into different orders, for example:

$$
\delta_{\theta} X=\left(\delta_{\theta} X\right)_{2}+\left(\delta_{\theta} X\right)_{1}+\left(\delta_{\theta} X\right)_{0},
$$

Similarly, we can decompose the perturbation of potential $V$ into different orders, for example:

$$
\delta_{\theta} V=\left(\delta_{\theta} V\right)_{2}+\left(\delta_{\theta} V\right)_{1}+\left(\delta_{\theta} V\right)_{0},
$$

We calculate the non commutative correction of two-point Green's function at the lowest order:

$$
\delta<\zeta\left(x_{1}\right) \zeta\left(x_{2}\right)>_{\theta}=-2 \operatorname{Re}\left(\int_{\eta_{0}}^{\eta} d \eta^{\prime} i<\zeta\left(x_{1}\right) \zeta\left(x_{2}\right)\left(\delta L_{\text {int }}+2\left(\delta_{\theta} V\right)_{2}\right)\left(\eta^{\prime}\right)>_{0}\right),
$$

Here, $k_{1}=k_{2}=k$ has been set to write the corrected power spectrum:

$$
\begin{aligned}
\delta P_{\theta}^{\zeta} & =\delta<\zeta\left(x_{1}\right) \zeta\left(x_{2}\right)>_{\theta} \\
& =\frac{1}{32 \varepsilon^{2}} \pi^{2} l_{p}^{4} \phi_{0}^{2} \dot{\phi}_{0}^{2} H\left[\left(-\frac{1}{k^{2}}-\frac{45}{8 k^{6}}+\frac{681}{16 c_{s}^{4} k^{10}}-\frac{333}{8 c_{s}^{6} k^{14}}\right) P_{X}\right. \\
& \left.+\left(\frac{c_{s}}{2 k^{2}}+\frac{5 \dot{\phi}_{0}^{2}}{8 k^{6}}\right) P_{X X}-\frac{n(n-1) \dot{\phi}_{0}^{n-1}}{4 H_{2} k^{2}} P_{V}+\frac{n(n-1) \dot{\phi}_{0}^{n-2}}{4 H^{2} k^{2}} P_{V X}\right] .
\end{aligned}
$$

According to Max Tegmark, the parallel universe is not a theory, but a theoretical prediction. 
Various models of the inflation universe depend on the choice of parameters, including the shape and initial conditions of the inflation energy density curve ${ }^{[21]-[23]}$.

Let's further expand our thinking space:

Before the creation of our universe, the whole world system of mult-verse was filled with infinite pseudo-vacuum oceans, where high-density energies converged. Every fluctuation of the initial vacuum implies the collective birth and death of particles.

The pseudovacuum, which accumulates extremely high density energy, forms negative pressure on the universe and then converts it into repulsive force, opening up a new space-time for the universe $^{[21]-[23]}$. With the rapid expansion of space-time, the Higgs field began to over-cool, and the pseudo-vacuum began to increase, causing a larger scale of expansion, leading to the increase of space-time. Through the non-linear feedback, the pseudo-vacuum increases continuously, and the initial gravitation and strong force separated from the high dimensional supergravity form a big rip-rebound cluster of quantum chaotic-reticulate distribution at macro and micro levels respectively with the expansion force caused by Higgs field, thus forming a great impetus of first suppression, later acceleration and last deceleration of the universe, and then causing the universe to enlarge exponentially.

Under the extreme conditions of the initial universe, in the a big rip-rebound cluster of quantum chaotic-reticulate distribution emerging between the initial gravity separated from quantum gravity and the expansive force caused by Higgs field, and then between the strong force separated from the grand unified gauge field and the expansive force caused by Higgs field, the basic matter unit successively becomes the arche-chaotic pulsator, quasi-spherical pulsator and spherical pulsator. Various wave modes of the arche-pulsator form various particles.

In the initial stage of the creation of the universe, the interaction between the expansive force caused by the Higgs field and all the internal gravitational forces, as well as the interaction between the expansive force caused by the Higgs field and all the internal strong forces, become a big impetus for the acceleration or deceleration of the cosmic inflation, thus becoming an extremely important factor that can not be ignored. The higher the density of cosmic energy, the higher the degree of synergy of the interaction system. This ultra-synergy between particles and the universe creates a sudden force of cosmic inflation, which leads to the exponential increase of cosmic inflation. The faster the universe cools, the stronger the power of space-time.

At the end of the cosmic explosion, the intensity of gravitational wave decreases rapidly, the electromagnetic interaction is separated from the weak interaction, and the cosmic inflation ends. At this moment, the temperature is $10^{28} \mathrm{~K}$, the time of cosmic creation is $10^{-32} \mathrm{sec}$, and the zero energy vacuum is formed. With the expansion of asymmetric space-time, the expansion continues until $10^{-12}$ sec of the creation of the universe, but the expansion of the universe in this period of time is in deceleration expansion, and ultimately the universe forms.

The universe today is not expanding in an inflationary way. In this way, it must have a mechanism to eliminate this very large effective cosmic constant, so that the expansion rate can change from an accelerated state to a slowed down state by gravity as it is today. One can expect that the symmetry between forces will eventually be destroyed when the universe inflates, just as supercooled water will eventually solidify. In this way, the extra energy of the unbroken symmetric state will be released and the universe will be re-heated to just below the critical temperature at which different forces are 
symmetrical. After that, the universe continued to expand and cool in the standard Big Bang mode.

\section{Basis of Ultra-Synergy Dynamics for Quantum phy-sphere}

In physics, the exploration of trying to approach the original and seek the grand unification is basically carried out in the category of gravity (covering classical gravity, quantum gravity and highdimensional super gravity). Up to now, one of the most profound problems in theoretical physics is to reconcile between general relativity and quantum mechanics. The former describes gravity and can be applied to large-scale structures (stars, planets, milky way), while the latter describes other three kinds of actions and reconciles basic forces at the micro scale.

An important lesson in general relativity teaches us that there is no fixed background of space-time, but in Newtonian mechanics and special relativity, space-time geometry is dynamic. Although it is easy to master in principle, it is the most difficult concept in general relativity, while the results it brings are quite far-reaching, and it has not been fully explored, even in terms of classical level. In a sense, general relativity can be regarded as a relational theory. In such a theory, the only physically important information is the relationship between different events in time and space.

While quantum mechanics depends on a fixed background, since it started from a fixed (non dynamic) background structure. In quantum mechanics, time is given at the beginning and is not dynamic, just like Newton's classical mechanics. In relativistic quantum field theory, as in classical field theory, Minkowski space-time is the fixed background of the theory. Finally, string theory is based on the expansion of quantum field theory, in which point particles are replaced by string objects, transferring in a fixed spatiotemporal background. Although the origin of string theory is in the study of quark confinement rather than quantum gravity, it was soon found that the spectrum of strings includes gravitons, and the "condensation" of several specific vibration modes of strings is equivalent to the modification of the original background.

The quantum field theory in the bending (non Minkowski) background, although not the quantum theory of gravity, also shows that some assumptions in the quantum field theory can not be extended to the curved space-time, let alone the perfect quantum gravity theory. In particular, when a vacuum exists, it is indicated that there is a dependency (Unruh effect) between the vacuum and the spatiotemporal path that the observer passes through. In addition, the concept of field seems to be more basic than the concept of particle (particle concept is taken as a convenient method to describe local interaction). The latter view is controversial and contradicts the quantum field theory developed in Minkowski space.

With the help of classical general relativity to describe the curved background space-time, physics defines the quantum field theory in the generalized curved space-time. Through this theory, it can be proved that black holes are also releasing particles through black body radiation, which is Hawking radiation, and it is possible to cause black holes to eventually evaporate through this mechanism. This shows that Hawking radiation plays a key role in the study of black hole thermodynamics. As the basis of particle physics in modern physics, quantum field theory is usually based on the flat Minkowski space-time, which is a very good approximation for the description of micro particles in a weak 
gravitational field like the earth. In some cases, the strength of the gravitational field is enough to affect the quantized matter, but it is not enough to require the gravitational field itself to be quantized.

Another attempt comes from the canonical quantization method in quantum theory. Applying the initial value form of general relativity, the result is the Wheeler Dwight equation (its function is similar to Schrodinger equation). Although the definition of this equation is not complete in general, but under the introduction of the so-called Ashtekar variable, we can get a very promising model from this equation: loop quantum gravity. In this theory, space is a kind of network structure called spin network, which evolves in discrete time. It depends on which properties in general relativity and quantum theory can be accepted to retain, and which energy level needs to be changed.

Cyclic quantum gravity or loop quantum gravity is the result of constructing the background independent quantum theory. Topological quantum field theory provides an example of background independent quantum field theory, but it has no local degrees of freedom and only a limited number of global degrees of freedom [99]-[101]. In this way, we can't describe 3+1-dimensional gravity; according to general relativity, even in vacuum gravity, there are local degrees of freedom. However, in the $2+1$ dimension, gravitational theory can be topological field theory, which has been successfully quantized by many methods, including spin network.

There are many other attempt theories of quantum gravity, such as dynamic triangulation, causal combination, torsion theory and quantum cosmology model based on path integration. All these tentative candidate theories still have major problems in form and concept that need to be solved, and they are all facing a common problem, that is, there is no way to verify the predictions of quantum gravity theory from experiments so far, and thus it is impossible to judge the correctness of the predictions by some differences between multiple theories. In this sense, the experimental observation of quantum gravity still needs to rely on the future cosmological observation and the related particle physics experiment to become possible.

This series of research attempts to transcend quantum gravity, supersymmetry and hypergravity, and make more in-depth and systematic exploration between high-dimensional hypergravity and high-dimensional hyperrepulsion, quantum gravity and quantum repulsion, and classical gravity and classical repulsion

It should be noted that this is a pursuit of the supreme goal: to develop a theoretical framework, not only with the help of experimental data can we infer the specific model of the observable universe. Like any scientific theory, there is no need for inflation to solve all possible problems. Although the new inflation model, like all scientific theories, is based on a set of assumptions and tries to understand these assumptions, this series of studies attempts to establish some deeper theories.

By the analysis and inference presented in this series, there is such a complex super-synergy mechanism:

In the Big Bang, inflation and expansion of the universe caused by pseudo-vacuum decay, Higgs mechanism and dark energy fluctuation, etc., the gravitational force, strong force and electrio-weak force separated from quantum gravity, supersymmetric unified force and gauge field force are very strong at the beginning (close to the intensity of quantum gravity). With the coordination of dark matter, they will form a strong attraction to the energy and matter around them, and ever-increasing gravitational sources, ever-increasing strong force sources and ever-increasing electro-weak sources should form a superimposed amplification effect, thus constructing a big rip-rebound cluster to the 
whole universe and the energy and matter around them, and then forming a great impetus role in which firstly super suppression is imposed, then super acceleration is imposed, finally super deceleration is imposed, for the inflation of the universe caused by pseudo-vacuum decay, Higgs mechanism and dark energy fluctuation. As a result, a flash-like super-inflation was created.

At the same time, in the big rip-rebound cluster of quantum chaotic reticulate distribution emerged in the early universe, the formation, movement and development of all particles (including superstring, superfilm, quark, etc.) can not be isolated events, nor can they be simple, random and idealized (absolutely not a free deduction of pure geometry). The most elementary unit of matter inevitably becomes a basic existence which evolves with the big rip-rebound cluster in the Big Bang, inflation and expansion of the universe. It must have gone through the evolution stages of chaotic pulsators, quasi-spherical pulsators and spherical pulsators, so that we can attribute the elementary unit model of to the arche-pulsator neither punctiform particles nor string objects.

On the basis of the previous discussions in this series, the quantum phy-sphere is proposed and studied in this paper is a system of all things accumulation centered on quantum gravitational sources. It is not only limited to the scope of quantum systems, but also involves the scope of macro-systems. It is not only limited to material or physical things, but also involves the complexity with a variety of elements. Its range of existence and evolution is generally slightly larger than the event horizon. In the quantum phy-sphere, the quantum gravi-source is not unique, but may be multiple. Before the inflation, the quantum universe could be regarded as the earliest quantum phy-sphere, and also a quantum phy-sphere with very short existence time. Each black hole itself is also a quantum gravi- spheres, and many of the binary star systems have become or will become quantum phy-spheres.

Quantum phy-spheres basically take the vicinity of black holes as their own sphere of influence, or with Schwarzschild radius as their own radius:

$R_{s}=\frac{2 G M}{c^{2}}$, here $G$ is the constant of gravitation, $M$ is the mass, $c$ is the speed of light.

The Schwarzschild radius formula is actually derived from the escape velocity formula of an object.

Taking the smallest black hole XTE J1650-500 discovered so far as an example, its radius is only $12 \mathrm{~km}$, but its mass is equivalent to 5 to 10 times the mass of the sun.

For example, the GCIRS 13E black hole is the same size as the entire European continent, but its mass is equivalent to 1,300 suns.

Taking HLX-1, a medium-sized black hole, as example, with a radius of 295,300 km, the size of four Jupiters side by side, but with a mass equivalent to 100,000 suns.

A supermassive black hole called Sagittarius A, located in the middle of the Milky Way, has a radius of 12,700,000 $\mathrm{km}$. This giant beast contains the mass of 4,300,000 suns!

Taking S5 0014+81, the largest black hole ever discovered in the universe, for example. Its radius is 1600 times the distance from the earth to the sun, and its mass is 40 billion times the mass of the sun.

In addition to various black holes, the original quantum universe can also be regarded as a quantum phy-sphere.

Under the framework of ultra-synergistic analysis of quantum universe proposed in this series, we have improved and expanded the path integral representation of quantum gravity through the previous work. For the quantum universe, we should consider not only the inwards-pull of quantum gravity, but 
also the outwards-push of the whole Big Bang. Here, we introduce a spatiotemporal metric of compact four-dimensional manifold which reflects both inwards-pull and outwards-push, and it can be written as

$$
d s^{2}=-\left(N_{B S}^{2}-N_{B S, i} N_{B S}^{i}\right) d t^{2}+2 N_{B S, i} d x^{i} d t+h_{B S, i j} d x^{i} d x^{j}
$$

here $N_{B S}$ is a time-lapse function reflecting the ultra-synergism, $N_{B S, i}$ is a shift function reflecting ultra-synergism, $h_{B S, i j}$ is an intrinsic metric on three-dimensional space-like Hypersurfaces under ultra-synergism, and $N_{B S}, N_{B S, i}, h_{B S, i j}$ are all functions of space-time coordinates of ultra-synergistic motion.

For the quantum universe, the coordinates of ultra-synergistic conformation space should be $h_{B S, i j}$ and matter field $\phi_{B S}$. The quantum universe is described by $\left(h_{B S, i j}, \phi_{B S}\right)$. Thus, the transition probability amplitude of the universe from a three-dimensional space-like hypersurface $h_{B S, i j}$ ( there is field $\phi_{B S}$ on it ) to a space-like hypersurface $h_{B S, i j}^{\prime}$ ( there is field $\phi^{\prime}{ }_{B S}$ on it ) can be expressed as

$$
<h_{B S, i j}^{\prime}, \phi_{B S}^{\prime} \mid h_{B S, i j}, \phi_{B S}>=\int \delta\left[g_{B S, \mu v}, \phi_{B S}\right] \exp \left(i I\left[g_{B S, \mu v}, \phi_{B S}\right]\right)
$$

here

$$
I\left[g_{B S, \mu v}, \phi_{B S}\right]=I_{B}\left[g_{B, \mu v}, \phi_{B}\right]+I_{S}\left[g_{\mu v}, \phi\right]+I_{B S}\left[g_{B S, \mu v}, \phi_{B S}\right]
$$

$I_{B}\left[g_{B, \mu v}, \phi_{B}\right]$ represents the outwards-push of the whole Big Bang.

$I_{S}\left[g_{\mu v}, \phi\right]$ represents the inwards-pull of quantum gravity.

$I_{B S}\left[\mathrm{~g}_{B S, \mu v}, \phi_{B S}\right]$ represents the ultra-synergism between the whole Big Bang and quantum gravity.

Similar to the treatment of general quantum systems, the pulsed function of ultra-synergism can be expressed as

$$
\Psi\left[h_{B S, i j}, \phi_{B S}\right]=N \int_{c} \delta g_{B S, \mu \nu} \delta \phi_{B S} \exp \left\{i I\left[g_{B S, \mu \nu}, \phi_{B S}\right]\right\}
$$

here, $N$ is the normalized constant, and the integral region $C$ is all the paths of the connective points $\left(h_{B S, i j}, \phi_{B S}\right)$ and the initial points in the ultra-synergistic configuration space. Under the full horizon criterion both inside and outside the universe, the ground-state pulsing function of ultra-synergistic system has the following form:

$$
\Psi\left[h_{B S, i j}, \phi_{B S}\right]=N \int_{C} \delta g_{B S, \mu \nu} \delta \phi_{B S} \exp \left\{-I\left[g_{B S, \mu \nu}, \phi_{B S}\right]\right\}
$$

It can be expected that the pulsating wave function ( 97 ) under ultra-synergism should satisfy a cosmic pulse dynamical equation similar to Schrodinger equation. The following equation belongs to this kind of equation, which can be regarded as an extension of Wheeler-De Witt equation.

Under the one-cycle (also called semi-classical WKB) approximation, formula ( 97 ) has a form:

$$
\Psi\left[h_{B S, i j}, \phi_{B S}\right]=N \sum_{i} B_{i} \exp \left(-I_{B S, c l}^{i}\right)
$$

here, $I_{B S, c l}{ }_{B S}$ is the $i$-th ultra-synergism which satisfies the principle of minimum action in classical physical form. $N$ is the normalized constant, and $B_{i}$ is the fluctuation of classical orbit.

By the uncertainty relation in quantum theory, the higher the energy is, the closer the interaction is., we always know the smaller scale physics by observing the scattering of higher energy scale. However, under the influence of gravity, this relationship no longer holds. In the analysis of existing 
theories, high-energy scattering beyond Planck energy scales $\left(10^{19} \mathrm{GeV}\right)$ will lead to black holes, and the Schwarzschild radius increases with the increase of mass. The higher the scattering energy is, the larger the black holes is, so that the closer interaction can not be observed. For general relativity, this is a difficult problem that can not be solved by renormalization ${ }^{[19]-[21]}$.

For the quantum phy-sphere proposed in this series, we introduce scalar functional:

$$
I=I_{B}+I_{S}+I_{B S}=\int\left(L_{B}+L_{S}+L_{B S}\right) \sqrt{-g} d^{4} x
$$

$I_{B}$ and $L_{B}$ represent the action and its Lagrangian function of the whole Big Bang (outwards-push) of the universe, respectively.

$I_{S}$ and $L_{S}$ represent the action and its Lagrangian functions of gravity (inwards-pull) respectively.

$I B S$ and $L_{B S}$ represent the interaction between the whole Big Bang and gravity and their Lagrangian functions, respectively.

The expression of $I_{S}$ and $L_{S}$ is taken as

$$
L_{S}=R, \quad L_{B}=-2 k L_{B} .
$$

here $k$ is Einstein gravitational constant, $k=8 \pi G / c^{4}$.

Here the variational principle can be expressed as: $\delta I=\delta\left(I_{B}+I_{S}+I_{B S}\right)=0$.

Physical studies of black holes show that there is a very large gravitation inside the black hole, and the closer it is to the center of the black hole, the stronger the gravitation is; there is a huge temperature difference between the visual interface and center of a black hole, and the non-equilibrium thermodynamic effect is very significant. Considering the non-equilibrium thermodynamic effect, and in order to overcome the singularity and information paradox of black hole, we can infer that under the combined action of great gravity and thermodynamic force, photons on the one hand take mass in the radial direction towards the black hole center, which results in a non-linear evolution; on the other hand, accelerate in the radial direction towards the black hole center, which may exceed the photons in the vacuum in velocity. (but not faster than photons in vacuum at each relative equilibrium time). Further, we can deduce that under the combined action of great gravity and thermodynamic forces, all physical particles on the one hand evolve nonlinearly in the radial direction towards the center of the black hole, on the other hand accelerate in the radial direction towards the center of the black hole. For a Kerr black hole with charged spherical collapse ( $E \neq 0$ ), the outer spatiotemporal geometry of matter is described by Reisner-Nordstron solution. Because Birkhoff theorem can be extended to ensure that Reisner-Nordstron space-time is the only spherical electrolysis.

\section{Distrib-Configuration and Mode Vector Dynamics}

For the quantum phy-sphere (QGS), we can consider the establishment of spin network space, quantum distribution configuration and gravitational field mode which represent the rationalization of space-time, and regard the ultra-synergistic distribution of quantum phy-sphere as an interaction system among the synergistic trend space, distribution configuration and interaction mode of all gravitational correlators in the quantum phy-sphere.

For the initial quantum universe, the arche-pulsator is used as the elementary unit of the quantum 
phy-sphere. For micro and small black holes, the fundamental particles are used as the elementary components of the quantum phy-sphere. For medium-sized and large black holes, the group of fundamental particles is used as the elementary unit of quantum phy-sphere.

For the ultra-synergistic distribution (instantaneous and real-time cluster distribution) of quantum gravitational circles, we consider all gravitational correlators in the sphere, including internal and external gravitational correlators, as well as direct and indirect correlators of quantum gravitational distribution, and consider their tendency towards the combination of gravitational collocating elements in different force fields, while the scope of gravitational allocation is the combination of gravitational collocating elements, including the gravitational collocating space, gravitational collocating base, gravitational collocating network, gravitational collocating environment, and so on.

For the spin network space as spatiotemporal rationalization, we can combine the spatiotemporal mode variables with the state variables of spin network, and analyze them from different aspects.

Here, it is assumed that the spin network space of the quantum phy-sphere has $A$ sides, namely, dimension (including integer dimension and fractal dimension, high dimension and low dimension) $\mu_{1}$, curvature $\mu_{2}$, spin quantum number $\mu_{3}$, contraction velocity $\mu_{4}$, spin angular momentum $\mu_{5}$, fluctuation level $\mu_{6}$, concentration degree $\mu_{7}$, action radius $\mu_{8}$, inhomogeneity $\mu_{9}$, range (distance scale) $\mu_{10}$, magnetic moment $\mu_{11}$, network node number $\mu_{12}$, concentration degree $\mu_{13}$ of network node distribution, competitiveness $\mu_{14}$ of network node distribution, etc.

Moreover, there are $d_{a}$ states in each side $a$ of spin network space, where $a=1,2, \cdots, A$. Thus, the $A$-dimensional trend space $M$ of the gravitational collocation with $A$ side is established. The gravitational collocation trend vector $r=\left\{r_{1}, r_{2}, \cdots, r_{A}\right\}$ of each elementary unit points to a lattice point in $M$ space, which indicates the tendency of this elementary unit to all $A$ sides. Therefore, there are $L=\prod_{a=1}^{A} d_{a}$ lattice points.

If the ultra-synergistic distribution of the quantum gravit-sphere is composed of $\Pi$ basic components, it can be divided into $G$ clusters (not limited to physical clusters). There are $\Pi_{\rho}$ basic components in the cluster $\mathscr{H}_{\rho}(\rho=1,2, \cdots, G)$. Therefore, $\Pi=\sum_{\rho=1}^{G} \Pi_{\rho}$, so the probability characteristics in $M$-space can be described by the basic components of each cluster.

When considering the formation and disappearance, or entry and exit of basic components, $\Pi_{\rho}$ is a function of time.

Generally speaking, in a gravitational collocation cluster $\mathscr{H}_{\rho}$ (not limited to physical clusters), The tendencies of $\Pi_{\rho}$ basic constituent units are different. We use $\pi_{\rho} r$ to denote the number of basic constituent units with the tend as $r=\left\{r_{1}, r_{2}, \cdots, r_{A}\right\}$ in $\mathscr{H}_{\rho}$. Therefore, $\Pi_{r}=\sum_{\rho=1}^{G} \pi_{\rho r}$, and the total number of trends $r$ in the ultra-synergistic distribution has the following relationship:

$$
\Pi=\sum_{r=1}^{L} \sum_{\rho=1}^{G} \pi_{\rho r}=\sum_{r=1}^{L} \Pi_{r}=\sum_{\rho=1}^{G} \Pi_{\rho}, \quad \text { here } \Pi_{\rho}=\sum_{r=1}^{L} \pi_{\rho r} .
$$


The distrib-configuration is now used to describe the macroscopic distribution of the quantum phy-sphere :

$$
\pi=\left\{\pi_{\rho l}\right\}=\left\{\pi_{11}, \pi_{21}, \cdots, \pi_{G 1}, \cdots, \pi_{\rho r}, \cdots, \pi_{1 L}, \pi_{2 L}, \cdots, \pi_{G L}\right\}
$$

It consists of $D=G L$ integer primitives with $\pi_{\rho r} \geq 0$. Distrib-configuration $\pi$ describes the trend distribution of the basic components in the distribution cluster $\mathscr{H}_{\rho}$ (not limited to physical clusters) of quantum phy-spheres.

Here, $\pi(t)=n(t,[\mathrm{SET}]), n(t)$ is the distrib-configuration of the quantum phy-sphere.

We use the motion point $\pi(t)=\left\{\pi_{\rho r}(t)\right\}=\left\{n_{\rho r}(t\right.$, [SET] $\left.)\right\}$ of $\Omega$ gravitational collocation space (spin network space) of D-dimensional distrib-configuration to express the time relationship of the distrib-configuration of the sampling quantum phy-sphere.

In the case of $\pi_{\rho r} \gg>1$ and properly tending to $\Pi_{0} \gg>1$, the quasi-continuous variable $X_{\rho r}=\pi_{\rho r} /$ $\Pi_{0}$ can be used.

On the establishment of the ultra-synergy distributing dynamics of quantum phy-spheres, we should consider not only the trend and configuration of all gravitational correlators of the ultra-synergy distribution, but also the mode of gravitational interaction.

We can describe the gravitational mode as a vector from $S$ sides. Here we consider:

$w_{1}$. strength, $w_{2}$. force range, $w_{3}$. mass density, $w_{4}$. the inverse ratio $\left(1 / S^{2}\right)$ to the square of the gravitational distance, $w_{5}$. quantization of gravitation, $w_{6}$. transfer of force, $w_{7}$. symmetry, $w_{8}$. inhomogeneity, $w_{9}$. conentration, $w_{10}$. fluctuation, $w_{11}$. synergy (cooperation and competitiveness), etc.

After defining the component $w_{\beta}(\beta=1,2, \cdots, S)$ from the $S$ sides of the gravitational mode of a quantum phy-sphere, we can introduce the S-dimensional gravitational mode space $\Omega$, so we can use the gravitational mode vector :

$$
w=y /[\operatorname{SET}]=\left(w_{1}, w_{2}, \cdots, w_{S} ;[\mathrm{SET}]\right) \in \Omega
$$

to describe the interaction mode of the quantum phy-sphere. Here, $w_{\beta}=y_{\beta} /[\mathrm{SET}](\beta=1,2, \cdots, S)$, $y_{\beta}$ is a component of the interaction mode vector of quantum phy-sphere.

Let $w_{1}$. strength, $w_{2}$. force range, $w_{3}$. mass density, $w_{4}$. the inverse ratio $\left(1 / S^{2}\right)$ to the square of the gravitational distance, $w_{9}$. conentration, $w_{11}$. competitiveness, then there are many types of gravitationalmodes (sub-modes), such as:

Mode type 001: $w[001]=\left(0.85 \sim 1.11,10 \mathrm{~cm} \sim 15 \mathrm{~cm}, 10^{8} \mathrm{~g} / \mathrm{cm}^{3} \sim 10^{9} \mathrm{~g} / \mathrm{cm}^{3}, 1 / 10^{2} \sim 1 /\right.$ $\left.10^{2}, 0.70-1.00,0.85-1.00\right) \in \Omega$;

Mode type 002: $w[002]=\left(0.70 \sim 0.85,30 \mathrm{~cm} \sim 40 \mathrm{~cm}, 10^{6} \mathrm{~g} / \mathrm{cm}^{3} \sim 10^{7} \mathrm{~g} / \mathrm{cm}^{3}, 1 / 25^{2} \sim 1 /\right.$ $\left.25^{2}, 0.85-0.85,0.85-1.00\right) \in \Omega$;

Mode type 003: $w[003]=\left(0.70 \sim 0.85,30 \mathrm{~cm} \sim 40 \mathrm{~cm}, 10^{6} \mathrm{~g} / \mathrm{cm}^{3} \sim 10^{7} \mathrm{~g} / \mathrm{cm}^{3}, 1 / 25^{2} \sim 1 /\right.$ $\left.25^{2}, 0.85-0.85,0.85-1.00\right) \in \Omega$;

By the interaction of spin network space, distrib-configuration and gravitational mode vector, a ultra-synergy distributed dynamics system of quantum phy-sphere can be established :

On the one hand, under the influence of a certain interaction mode vector $w$ and each basic component unit on the trend parameters $\lambda=\left\{\lambda_{1}, \lambda_{2}, \cdots, \lambda_{E}\right\}$ of the gravitational collocations range, a 
certain trend distribution and distrib-configurations $\pi(t)$ will be formed, and the trend change of the basic components causes the motion of $\pi(t)$; On the other hand, the change of the distribconfiguration will drive the action mode vector $w(t)$ into a new state. For example, the pattern of ultra-synergistic distribution may be changed by basic components for activities with a certain synthetic trend. At the same time, the changed interaction mode $w(t)$ will also react on the distrib-configuration $\pi(t)$. Under the simple assumptions, the gravitational collocation motion can terminate at the stationary state $\left\{\pi_{s t}, w_{s t}\right\}$. Generally speaking, however, the interaction between $\pi(t)$ and $w(t)$ can lead to highly complex coupling motions that are endless.

So far, the motion equation for the distrib-configuration of the quantum phy-sphere still does not contain all the complex factors of the ultra-synergy distributed dynamics. The above model only describes the movement of complex processes (i.e. the change of group trend) caused under the action mode vector $w$ and the trend parameter $\lambda$. Sometimes $w$ and $\lambda$ can be regarded as constants or slow-varying functions of time in discussion. The reciprocal transfer probability or drift and fluctuation coefficients (through parameters $w$ and $\lambda$ ) are slow variable functions of constant or time.

If they are constants, then the master equation or the Fokker-Planck equation can be used to prove (for example, through the famous Boltzmann H-theorem) that all the distributions will eventually enter the stationary distribution $W(x)$. In stationary state, the motion of the sampled quantum phy-sphere is only caused by the stationary fluctuation near the probability peak. This motion represents a "stationary distribution" under the condition of a stable state and interaction mode.

In general, the ultra-synergy distributed dynamical system of the quantum phy-sphere should be composed of the interaction among the distrib-configuration $x(t) \in \mathscr{F}$, the action mode vector $w(t) \epsilon$ $\Omega$ and the trend parameters $\lambda(t)=\left\{\lambda_{1}(t), \lambda_{2}(t), \cdots, \lambda_{E}(t)\right\} \in \mathscr{L}$ (where the trend parameters are expressed as the elements of the $E$-dimensional trend space).

When we discuss their coupling through Langevin's equation, we must treat variables as

$$
\{x(t), w(t), \lambda(t)\} \in \mathscr{F} \oplus \Omega \oplus \mathscr{L}=\mathscr{M}
$$

Now, the whole space of dynamic variables includes not only the mode vector $w(t)$ and the trend parameter $\lambda(t)$, but also the distrib-conformation variable $x(t)$, where the structure of motion equations of $w(t)$ and $\lambda(t)$ is discussed, and the states of quantum phy-spheres under various conditions are reflected in the model.

All the gravitational correlators of the ultra-synergy distributed dynamical system of the quantum phy-sphere will collectively "drive" the vectors of the interaction mode to generate motion, so that the time derivatives of $w(t)$ should consist of the superposition of these competitive partial derivatives:

$$
\frac{d w_{\beta}}{d t}=\left(\frac{\partial w_{\beta}}{\partial t}\right)_{1}+\left(\frac{\partial w_{\beta}}{\partial t}\right)_{2}+\cdots+\left(\frac{\partial w_{\beta}}{\partial t}\right)_{\Omega}, \quad \beta=1,2, \cdots, S
$$

here each item represents a function. In general, it depends on the nonlinear action of mode vector $w$ $(t)$, distrib-configuration $x(t)$ and some control parameter $\lambda(t)$.

Similarly, the time derivative of the trend parameter $\lambda(t)$, which determines the formula of the transition probability, represents the complex role of the tendency to change the interaction mode, and is also composed of the superposition of the competitive partial derivatives: 


$$
\frac{d \lambda_{l}}{d t}=\left(\frac{\partial \lambda_{l}}{\partial t}\right)_{1}+\left(\frac{\partial \lambda_{l}}{\partial t}\right)_{2}+\cdots+\left(\frac{\partial \lambda_{l}}{\partial t}\right)_{\Gamma}, \quad l=1,2, \cdots, E
$$

Here, the partial derivative denotes the change of action mode vector $w(t)$ caused by each trend, which will cause the change of distrib-configuration, while the new configuration in turn drives action mode $w(t)$ into the next new state, and so on. So far, the random force terms have been neglected in the formulas ( 105 ) and ( 106 ). When the random force terms are added, the formulas ( 105 ) and ( 106 ) become Langevin equations, and the statistical description of $x(t)$ is obtained. A multi-dimensional periodic or quasi-periodic or even chaotic motion of $\mathscr{H} \oplus \Omega \oplus \mathscr{L}=\mathscr{M}$ is a description of the average time development of the interaction mode, the trend parameters and the distrib-configuration. Of course, there are also the nonlinear coupled differential equation system of "limit cycles" or "singular attractors", whose solutions just show the characteristics of quasi-periodic or chaotic.

If the dynamic equation of the trend parameter is established by coupling the trend parameter with the interaction mode, the second-order trend parameter must be introduced. If the latter is regarded as a dynamic variable, an endless infinite coupling will be obtained; the dynamic equation of n-order linear parameters includes $(n+1)$ order trend parameters $(n=1,2, \cdots)$.

For drift coefficient $K_{j}(x)$ and transfer probability $W[K, \pi]$, we can get the equation of specific average value $\tilde{\pi}_{\rho i}$.

Neglecting the process of creation and extinction, we only consider the process described by transition probability (such as transition probability $p_{j i}^{\rho}$ and $p_{i}^{\beta \rho}$ ) between trend and cluster (not limited to physical cluster). For simplicity, the following approximate closed mean value equations are given:

$$
\begin{aligned}
\frac{d \tilde{\pi}_{\gamma \lambda}}{d t}= & \left\{\sum_{i=1}^{L} \tilde{\pi}_{\lambda \gamma} p_{\lambda i}^{\gamma}(\tilde{\pi}, w, \lambda)-\sum_{j=1}^{L} \tilde{\pi}_{\gamma \lambda} p_{j \lambda}^{\gamma}(\tilde{\pi}, w, \lambda)\right\} \\
& +\left\{\sum_{\rho=1}^{G} \tilde{\pi}_{\rho \lambda} p_{\lambda}^{\gamma \rho}(\tilde{\pi}, w, \lambda)-\sum_{\rho=1}^{G} \tilde{\pi}_{\gamma \lambda} p_{\lambda}^{\rho \gamma}(\tilde{\pi}, w, \lambda)\right\}
\end{aligned}
$$

In General, the transition probability here depends not only on the distrib-configuration $\tilde{\pi}$ and trend parameter $\lambda$, but also on the interaction mode vector $w$.

The conserved number of the first term on the right side of equation ( 107 )

$$
\Pi_{\rho}=\sum_{i=1}^{L} \pi_{\rho i}, \quad \rho=1,2, \cdots, G
$$

the conserved number of the second term is $\Pi_{i}=\sum_{\rho=1}^{G} \pi_{\rho i}, i=1,2, \cdots, L$.

However, if $\tilde{\pi}_{\gamma \lambda}$ change in the two effects, there only is the total 


$$
\Pi=\sum_{\rho=1}^{G} \sum_{i=1}^{L} \pi_{\rho i}, \quad \rho=1,2, \cdots, G ; \quad i=1,2, \cdots, L
$$

to be conserved.

This problem is described as follows: suppose that there are $L$ trends and $G$ clusters (not limited to physical clusters) (where $L \gg 1, G \gg 1$ ), there are $D=G L$ the descriptions of dynamics for the ultra-synergy. The average value $\tilde{\pi}_{\rho i}$ of distrib-conformation variable $\left\{\pi_{\rho i}\right\}$ is still a complex variable of "fine grains". Therefore, the average equation of several total variables are derived from ( 107 ).

These total variables should be functions of $D$ distrib-conformation variables $\left\{\pi_{\rho i}\right\}$. Although the basic components of ultra-synergy distributed dynamics are fewer than $\left\{\pi_{\rho i}\right\}$, we should be cautious in studying the overall state of ultra-synergy distributed dynamics.

Assuming that the general form of the total variable is

$$
H_{q}=\sum_{i=1}^{L} \sum_{\rho=1}^{G} h_{\rho i} \pi_{\rho i}
$$

Here, $h^{q}{ }_{\rho i}(q=1,2, \cdots, Q)$ is the real coefficient, that is to say, the weighted average of proper tendency is carried out on the basic constituent units of clusters with some tendency (not limited to physical clusters), and the basic constituent units $\Pi_{\rho}$ and $\Pi_{i}$ are the special cases of the total variables. For example, the total variables $P_{1}$ and $P_{2}$ represent the overall state of the "complex state" from "consistent" to "conflicting" between two interacted quantum phy-spheres $\Xi_{j}$ in competition.

If $P_{j}$ represents a positive (negative) value dominated by consistency (conflict), it will obviously tend to the weight defined by $P_{i}$. For example, positive weight $h_{\rho i}^{j}>0$ classifications tend to be hierarchical ( $\rho i$ ) such as "cooperation" and "support", and negative weight $h_{\beta k}^{j}<0$ classifications tend to be hierarchical ( $\beta i$ ) such as "arbitrariness" and "vertical communication".

Consider how to derive the non-closed motion equation of average value $\bar{H}_{q}$. By $h_{j k}^{q}$ multiplying ( 107 ) and taking the sum of $\gamma$ and $k$, we get

$$
\begin{aligned}
\frac{d}{d t} \sum_{\gamma, k}^{G, L} h_{\gamma k}^{q} \tilde{\pi}_{\gamma k} \Rightarrow \frac{d}{d t} \tilde{H}_{q} & =\sum_{\gamma, k}^{G, L} h_{\gamma k}^{q}\left[\sum_{i=1}^{L} \tilde{\pi}_{\gamma i} p_{k i}^{\gamma}(\tilde{\pi}, w, \lambda)-\sum_{j=1}^{L} \tilde{\pi}_{\gamma k} p_{j k}^{\gamma}(\tilde{\pi}, w, \lambda)\right] \\
& +\sum_{\gamma, k}^{G, L} h_{\gamma k}^{q}\left[\sum_{\rho=1}^{G} \tilde{\pi}_{\rho k} p_{k}^{\gamma \rho}(\tilde{\pi}, w, \lambda)-\sum_{\rho=1}^{G} \tilde{\pi}_{\gamma k} p_{k}^{\rho \gamma}(\tilde{\pi}, w, \lambda)\right]
\end{aligned}
$$

The right side of the upper formula depends not only on $\tilde{H}_{q}$, but also on $\tilde{\pi}_{\gamma i}$, so that (109) is not closed.

In order to obtain the closed equation of $\tilde{H}_{q}$, new assumptions and approximations must be made.

Firstly, it is assumed that the transition probabilities $p_{k i}^{\gamma}$ and $p_{i}^{\gamma \rho}$ are the positive exponential expression, 
and only the linear terms of Taylor expansion for $\pi$ and $w$ are preserved in the exponent.

$$
\begin{aligned}
& p_{k i}^{\gamma}(\tilde{\pi}, w, \lambda)=v \exp \left[\delta\left(\begin{array}{l}
\gamma \\
k i
\end{array}\right)+\sum_{\beta, j}^{G, L} \lambda\left(\begin{array}{c}
\gamma \\
k i
\end{array} \mid \beta_{j}\right) \pi_{\beta j}+\sum_{m}^{S} \sigma\left(\begin{array}{l}
\gamma \\
k i
\end{array} \mid m\right) w_{m}\right] \\
& p_{i}^{\gamma \rho}(\tilde{\pi}, w, \lambda)=\mu \exp \left[\delta\left(\begin{array}{c}
\gamma \rho \\
i
\end{array}\right)+\sum_{\beta, j}^{G, L} \lambda\left(\begin{array}{c}
\gamma \rho \\
i
\end{array} \mid \beta_{j}\right) \pi_{\beta j}+\sum_{m}^{S} \sigma\left(\begin{array}{l}
\gamma \rho \\
i
\end{array} \mid m\right) w_{m}\right]
\end{aligned}
$$

Here, coefficients $\left.v, \delta\left(\begin{array}{l}\gamma \\ k i\end{array}\right), \lambda\left(\begin{array}{l}\gamma \\ k i\end{array} \mid \beta_{j}\right), \sigma\left(\begin{array}{l}\gamma \\ k i\end{array}\right) m\right)$ as well as $\mu, \delta\left(\begin{array}{l}\gamma \rho \\ i\end{array}\right), \lambda\left(\begin{array}{l}\gamma \rho \\ i\end{array} \mid \beta_{j}\right)$ and $\sigma\left(\begin{array}{l}\gamma \rho \\ i\end{array} \mid m\right)$ are trend parameters that are not further explained here.

If the total variable approximately obeys the uniformly closed sub-dynamics and the transition probability depends only on $\left\{\pi_{\rho i}\right\}$ by the total variable, it can be taken as a self-consistent form. This assumption requires special trend parameters

$$
\lambda\left(\begin{array}{l}
\gamma \\
k i
\end{array} \mid \beta_{i}\right)=\sum_{q}^{Q} \omega\left(\begin{array}{l}
\gamma \\
k i
\end{array}\right) q h_{\beta_{j}}^{q}, \quad \lambda\left(\begin{array}{l}
\gamma \rho \\
i
\end{array} \mid \beta_{i}\right)=\sum_{q}^{Q} \omega\left(\begin{array}{l}
\gamma \rho \\
i
\end{array} \mid q\right) h_{\beta_{j}}^{q}
$$

so as to obtain

$$
\begin{aligned}
& p_{k i}^{\gamma}(\tilde{\pi}, w, \lambda)=p_{k i}^{\gamma}[H, w, \lambda]=v \exp \left[\delta\left(\begin{array}{c}
\gamma \\
k i
\end{array}\right)+\sum_{q=1}^{H} \omega\left(\begin{array}{c}
\gamma \\
k i
\end{array} \mid q\right) H_{q j}+\sum_{m}^{S} \sigma\left(\begin{array}{l}
\gamma \\
k i
\end{array} \mid m\right) w_{m}\right] \\
& p_{i}^{\gamma \rho}(\tilde{\pi}, w, \lambda)=p_{i}^{\gamma \rho}[H, w, \lambda]=\mu \exp \left[\delta\left(\begin{array}{c}
\gamma \rho \\
i
\end{array}\right)+\sum_{q=1}^{H} \omega\left(\begin{array}{c}
\gamma \rho \\
i
\end{array} \mid q\right) H_{q}+\sum_{m}^{S} \sigma\left(\begin{array}{l}
\gamma \rho \\
i
\end{array} \mid m\right) w_{m}\right]
\end{aligned}
$$

$p_{k i}^{\gamma}$ and $p_{i}^{\gamma \rho}$ depend not only on $p_{k i}^{\gamma}[\mathrm{H}, \mathrm{w}, \lambda]$ and $p_{i}^{\gamma \rho}[\mathrm{H}, \mathrm{w}, \lambda]$, but also on each $\tilde{\pi}_{\rho j}(\rho=1,2$, $\cdots, G ; j=1,2, \cdots, L)$, so the closed equation of $\tilde{H}_{q}$ can not be derived. However, as long as the function of the mean value $\tilde{H}_{q}(t)$ is used, we can be proved $\tilde{\pi}_{\rho i}(t)=\tilde{\pi}_{\rho i}[\tilde{H}(t)]$, it can be transformed into an approximate closed equation:

$$
\begin{aligned}
\frac{d}{d t} \tilde{H}_{q}(\mathrm{t}) & =\sum_{\gamma, k}^{G, L} g_{\gamma k}^{q}\left[\sum_{i=1}^{L} \tilde{\pi}_{\gamma i}(\tilde{H}) p_{k i}^{\gamma}(\tilde{H}, w, \lambda)-\sum_{j=1}^{L} \tilde{\pi}_{\gamma k}(\tilde{H}) p_{j k}^{\gamma}(\tilde{H}, w, \lambda)\right] \\
& +\sum_{\gamma, k}^{G, L} g_{\gamma k}^{q}\left[\sum_{\rho=1}^{G} \tilde{\pi}_{\rho \mathrm{k}}(\tilde{H}) p_{k}^{\gamma \rho}(\tilde{H}, w, \lambda)-\sum_{\rho=1}^{G} \tilde{\pi}_{\gamma k}(\tilde{H}) p_{k}^{\rho \gamma}(\tilde{H}, w, \lambda)\right]
\end{aligned}
$$

By substituting the formulas ( 113 ) and ( 114 ) into ( 109 ), the reduced set of the mean value $\tilde{H}_{q}(t)$ ( $q=1,2, \cdots, Q$ ) of the total variable can be obtained. In order to determine the function $\tilde{\pi}_{\rho j}[\tilde{H}]$, the basic method of statistical physics is used to study the catastrophe in ultra-synergistic distribution. 
Obviously, the distrib-configuration $\pi_{\rho i}(t)$ of the quantum phy-sphere should be consistent with the known value of $H_{q}(t)$, and the mean value $\tilde{H}_{q}(t)$ of the total variable at moment $t$ should be meet

$$
\sum_{\rho, i}^{G, L} h_{\rho i}^{q} \pi_{\rho i}(t)=\tilde{H}_{q}(t), \quad q=1,2, \cdots, Q
$$

Nowadays, the distrib-configuration $\left\{\pi_{\rho i}\right\}$ is a macroscopic configuration formed by many microscopic configurations. Microstructure refers to the distribution of clusters (not only physical clusters) in $G L$ "boxes", $(\rho, i)$ denotes clusters $\rho$ (not only physical clusters) and trends $i$. The number $M\left(\left\{\pi_{\rho i}\right\}\right)$ of micro-configurations corresponding to a macro configuration $\left\{\pi_{\rho i}\right\}$ is

$$
M\left(\left\{\pi_{\rho i}\right\}\right)=\pi ! / \prod_{\rho, i}^{G, L} \pi_{\rho i} !
$$

Furthermore, the mean value $\tilde{H}_{q}(t)$ of total variables (t) is only a slowly varying time track, compared with the fast transfer between various micro-configurations. At this time, it can be assumed that the instantaneous equilibrium transfer is achieved among the micro configurations at the determined value $\tilde{H}_{q}(t)$, while the magnitude order of the transfer probabilities among all the micro configurations satisfying the constraint condition (116) is the same. Under this approximation condition, the instantaneous mean value $\tilde{H}_{q}(t)$ of the total variable is equal, and all kinds of microscopic configurations have the same weight. Therefore, at a certain $\tilde{H}_{q}(t)$, the probability of macrodistribution configuration $\left\{\pi_{\rho i}\right\}=\pi$ is proportional to the number of realizable micro-configurations, that is to say,

When $\pi \equiv\left\{\pi_{\rho i}\right\}$ and ( 116 ) are consistent,

$$
p_{H}(\pi)=M(\pi) / \sum_{\{\pi\}}^{(H)} M(\pi)
$$

When $\pi \equiv\left\{\pi_{\rho i}\right\}$ and ( 116$)$ are inconsistent,

$$
p_{H}(\pi)=0
$$

Here the summation $\sum_{\{\pi\}}^{(H)}$ of all macroscopic distrib-configurations $\pi \equiv\left\{\pi_{\rho i}\right\}$ is the same as that of ( 116 ). Under the constraint of ( 116 ), by using ( 118 ) and by definition

$$
\tilde{\pi}_{\beta k}(t)=\sum_{\{\pi\}} \pi_{\beta k} p_{H(t)}(\pi)
$$

In principle, the average value $\tilde{\pi}_{\rho i}(t)$ can be calculated. Since the probability distribution ( 118 ) 
depends on $\tilde{H}_{q}(t)$, the average value $\tilde{\pi}_{\beta k}(t)$ of the distrib-configuration becomes a function of the average value $\tilde{H}_{q}(t)$ of the total variable.

In fact, it is troublesome to calculate the average value ( 119 ) by this method, but under the constraint ( 116 ), the probability distribution ( 118 ) has an obvious or a steep maximum at $\left\{\hat{\pi}_{\rho i}\right\}$.

Because the steepness (approximation) of the mean value $\tilde{\pi}_{\beta k}$ of the maximum $p_{H}\left(\left\{\pi_{\rho i}\right\}\right)$ is the same as the maximum $\left\{\hat{\pi}_{\rho i}\right\}$ of $p_{H}\left(\left\{\pi_{\rho i}\right\}\right)$, we can calculate $\tilde{\pi}_{\beta k}$ conveniently. Using Stirling's formula $\ln (n !) \approx n \ln (n)-n$, the following relations can be obtained

$$
\ln \left(\frac{\pi !}{\prod_{\rho, i}^{G, L} \pi_{\rho i} !}\right) \approx \Pi \ln (\Pi)-\Pi-\sum_{\rho, i}^{G, L}\left[\pi_{\rho i} \ln \left(\pi_{\rho i}\right)-\pi_{\rho i}\right]
$$

The above formula must be very large when ( 116 ) and the conditions $\sum_{\rho, i}^{G, L} \pi_{\rho i}=\Pi$ are met. Using the variational principles

$$
\delta\left\{-\sum_{\rho, i}^{G, L}\left[\pi_{\rho i} \ln \left(\pi_{\rho i}\right)-\pi_{\rho i}\right]+\mu_{0}\left(\sum_{\rho, i}^{G, L} \pi_{\rho i}-\Pi\right)+\sum_{q=1}^{Q} \mu_{q}\left[\sum_{\rho, i}^{G, L} h_{\rho i}^{q} \pi_{\rho}-\tilde{H}_{q}(t)\right]\right\}=0
$$

here $\mu_{0}$ and $\mu_{q}(q=1,2, \cdots, Q)$ is a Lagrangian multiplier, and each $\pi_{\rho}$ can vary independently. We derive by Variation

$$
\text { when } \begin{aligned}
& \pi_{\rho i}=\hat{\pi}_{\rho} \text { and } \hat{\pi}_{\rho}=\exp \left(\mu_{0}+\sum_{q=1}^{Q} \mu_{q} h_{\rho i}^{q}\right) \\
& \delta \pi_{\rho i}\left[-\ln \left(\pi_{\rho i}\right)+\mu_{0}+\sum_{q=1}^{Q} \mu_{q} h_{\rho i}^{q}\right]=0
\end{aligned}
$$

here, Lagrange multipliers $\mu_{0}$ and $\mu_{q}$ are treated as functions of $\Pi$ and $\tilde{H}_{q}(t)(q=1,2, \cdots, Q)$, i.e.

$$
\mu_{0}=\mu_{0}\left(\Pi, \tilde{H}_{q}(\mathrm{t})\right) \text { and } \mu_{\mathrm{q}}=\mu_{\mathrm{q}}\left(\Pi, \tilde{H}_{q}(\mathrm{t})\right) .
$$

Under the constraints :

$$
\sum_{\rho, i}^{G, L} \hat{\pi}_{\pi i} \equiv \sum_{\rho, i}^{G, L} \exp \left(\mu_{0}+\sum_{q=1}^{Q} \mu_{q} h_{\rho i}^{q}\right)=\Pi
$$




$$
\sum_{\rho, i}^{G, L} h_{\rho i}^{q} \hat{\pi}_{\pi i} \equiv \sum_{\rho, i}^{G, L} h_{\rho i}^{q} \exp \left(\mu_{0}+\sum_{q=1}^{Q} \mu_{q} h_{\rho i}^{q}\right)=\tilde{H}_{q}(t)
$$

By putting Lagrange multipliers $\mu_{0}\left(\Pi, \tilde{H}_{q}(\mathrm{t})\right)$ and $\mu_{\mathrm{q}}\left(\Pi, \tilde{H}_{q}(\mathrm{t})\right)$ into ( 122$)$, the most probable distrib-configurations $\left\{\hat{\pi}_{\rho \mathrm{i}}\right\} \approx\left\{\tilde{\pi}_{\rho \mathrm{i}}\right\}$ as $\Pi$ and $\tilde{H}_{q}(t)$ functions are obtained. By putting the function $\hat{\pi}_{\rho \mathrm{i}}(\Pi, \tilde{\mathrm{H}})$ into formula ( 115$)$, the motion equation of $\tilde{H}_{q}(t)$ is obtained.

In order to illustrate the similarity with statistical physics, a quantity similar to the entropy function is introduced :

$$
\hat{S}(t)=-\sum_{\rho, i}^{G, L}\left[\hat{\pi}_{\rho i}(t) \ln \left(\hat{\pi}_{\rho i}(t)-\hat{\pi}_{\rho i}(t)\right)\right]
$$

After differentiating the upper formulas and putting it into ( 122 ), we can get the result.

$$
\begin{aligned}
\frac{d \hat{S}(t)}{d t} & =-\sum_{\rho, i}^{G, L} \frac{d \hat{\pi}_{\rho i}}{d t} \ln \left(\pi_{\rho i}\right) \\
& =-\sum_{\rho, i}^{G, L} \frac{d \hat{\pi}_{\rho i}}{d t}\left(\mu_{0}+\sum_{q=1}^{Q} \mu_{q} h_{\rho i}^{q}\right)=-\mu_{0} \frac{d \Pi}{d t}-\sum_{q=1}^{Q} \mu_{q} \frac{d \hat{H}_{q}(t)}{d t}
\end{aligned}
$$

By introducing "the dynamic potential of ultra-synergy distribution"

$$
\hat{T}(t)=\hat{S}+\mu_{0} \Pi+\sum_{q=1}^{Q} \mu_{q} \hat{H}_{q}
$$

and its equivalent formula

$$
\frac{d \hat{T}(t)}{d t}=\Pi \frac{d \mu_{0}}{d t}+\sum_{q=1}^{Q} \hat{H}_{q}(t) \frac{d \mu_{q}}{d t}
$$

Then ( 115 ) is put into the right side of ( 125 ) or ( 127 ), and the motion tracks of the ultra-synergistic distributed dynamic entropy and the ultra-synergistic distributed dynamic potential are obtained.

\section{Computational Example of Cluster-Inflating Configuration}

Now, we can describe the process of the universe from 13.8 billion years to just one nanosecond in a way that is as close to the arche as possible. Here, we consider the basic quantities involved in the ultimate theory: energy, density, temperature, spatiotemporal dimension, scale, expansion rate, volume.

At the moment $(t=0)$ of the Big Bang of the universe, the temperature reached its maximum: $T_{\max } \gg$ plan temperature $\left.\left.1.416833(85) \times 10^{32} \mathrm{~K} ; l<l_{p}=10^{-33} \mathrm{~cm}, E\right\rangle 1.2 \times 10^{84} \mathrm{~J}, \rho\right\rangle$ density $10^{94}$ $\mathrm{g} \cdot \mathrm{cm}^{-3}$. From the beginning ( $t=0$ ) of the Big Bang to $10^{-44} \mathrm{~s}$ (planck time) after the Big Bang: the universe is in the super-symmetric grand unification stage. In the Planck time of one unit after the Big Bang, the density of the universe is about one unit of Planck density: 


$$
\rho_{P}=\frac{m_{P}}{l_{P}^{3}}=\frac{\hbar t_{P}}{l_{P}^{5}}=\frac{c^{5}}{\hbar G^{2}} \approx 5.155 \times 10^{96} \mathrm{~kg} \cdot \mathrm{m}^{-3} .
$$

At this time, the temperature is about the Planck temperature $1.416833(85) \times 10^{32} \mathrm{~K}$. Planck's law states that energy cannot continue to increase, otherwise it will produce unimaginable rays. The limit point is about $1.416833(85) \times 10^{32} \mathrm{~K}$, commonly known as Planck temperature.

According to current physical cosmology, this is the temperature of the first instant of the Big Bang (the first unit Planck time):

$$
T_{P}=\frac{m_{P} c^{2}}{\kappa}=\sqrt{\frac{\hbar c^{5}}{G \kappa^{2}}} \approx 1.416833(85) \times 10^{32} \mathrm{~K}
$$

here, $m_{P}$ is Planck mass, $c$ is the speed of light in vacuum, $\hbar$ is reduced Planck constant (also known as Dirac constant), $\kappa$ is Boltzmann constant, $G$ is gravitational constant, and the last two uncertainties (standard deviation) are in brackets.

Before the explosion $\left(T<10^{-36} \mathrm{~s}\right)$, the universe expanded at the square of the speed of light:

$$
\begin{aligned}
& E_{u-p h y}=E_{P}=\sqrt{\frac{\hbar c^{5}}{G}} \approx 1.956 \times 10^{9} \mathrm{~J} \approx 1.22 \times 10^{19} \mathrm{GeV} \approx 0.5433 \mathrm{MWh} \\
& \rho_{u-p h y}=\rho_{P}=\frac{m_{P}}{l_{P}^{3}}=\frac{\hbar t_{P}}{l_{P}^{5}}=\frac{c^{5}}{\hbar G^{2}} \approx 5.155 \times 10^{96} \mathrm{~kg} \cdot \mathrm{m}^{-3}
\end{aligned}
$$

The creation period of the universe $\left(10^{-44} \mathrm{~s} \sim 10^{-38} \mathrm{~s} ; 0.999999 \times 10^{-38} \mathrm{~s}\right)$

$$
m=E / c^{2}=1.2 \times 10^{84} \mathrm{~J} /\left(3 \times 10^{10} \mathrm{~cm} \cdot \mathrm{s}^{-1}\right)^{2}=0.136 \times 10^{66} \mathrm{~kg}
$$

After the super-inflation, the scale of the universe increases by $10^{24}$ times $\left(10^{24} \times 10^{-33} \mathrm{~cm}=10\right.$ ${ }^{-9} \mathrm{~cm}$ ), the volume of the universe $V_{u-p h y}=4.222 \times 10^{-99} \mathrm{~cm}$, and the expansion is $10^{70}$ times.

The super-inflation occurred between $10^{-36} \mathrm{sec}$ and $10^{-32} \mathrm{sec}$ after the Big Bang of the universe. During the infaltion of the universe, the temperature dropped from $10^{27} \mathrm{~K}$ to $10^{22} \mathrm{~K}$.

As two examples, Table 1 (Li Zongcheng, 2019) gives cluster-inflating configurations in different spin network spaces and arche-state vectors, and Table 2 (Li Zongcheng, 2019) gives cluster-inflating configurations in different action mode vectors and spin network spaces.

When the universe is at an extremely high temperature of $10^{32} \mathrm{~K}$ ( $10^{26}$ times higher than the temperature of our Sun), the gravity is separated from the other forces of the super-gravity. When the universe enters $t=10^{-38} \mathrm{~s}, T=1.4150 \times 10^{29} \mathrm{~K}, E_{u-p h y}=2.7488 \times 10^{66} \mathrm{GeV}, m_{u-p h y}=0$, $R=9.9999 \times 10^{-18} \mathrm{~cm}, \rho_{u-p h y}=1.07355 \times 10^{84} \mathrm{~kg} \cdot \mathrm{cm}^{-3}, V_{u-p h y}=4.18654 \times 10^{-51} \mathrm{~cm}^{3}$,

$$
\begin{aligned}
E_{u-p h y} & =1.45 \times 10^{18} \mathrm{GeV} \times\left(\frac{4}{3} \pi R^{3} / \frac{4}{3} \pi r_{\text {planck }}^{3}\right) \\
& =1.45 \times 10^{18} \mathrm{GeV} \times\left[\left(9.999 \times 10^{-18} \mathrm{~cm}\right)^{3} /\left(\frac{1}{2} \times 1.616 \times 10^{-33} \mathrm{~cm}\right)^{3}\right]
\end{aligned}
$$




$$
\begin{aligned}
& \quad=1.45 \times 10^{18} \mathrm{GeV} \times \frac{999.997 \times 10^{-54} \mathrm{~cm}}{0.52751 \times 10^{-99} \mathrm{~cm}}=2.7488 \times 10^{66} \mathrm{GeV} \\
& R=\frac{1}{2} \times l_{\text {planck }}+c^{2} \times 10^{-43} \mathrm{~s} \\
& =\frac{1}{2} \times 1.616 \times 10^{-33} \mathrm{~cm}+\left(3 \times 10^{10} \mathrm{~cm} \cdot \mathrm{s}^{-1}\right)^{2} \times 10^{-43} \mathrm{~s}
\end{aligned}
$$

\begin{tabular}{|c|c|c|c|c|c|}
\hline & $\begin{array}{l}\text { spin net space } \\
\qquad S N 01 \\
\left(10^{-44} \mathrm{sec}\right)\end{array}$ & $\begin{array}{c}\text { spin net space } \\
\text { SN } 06 \\
\left(10^{-39} \mathrm{sec}\right)\end{array}$ & $\begin{array}{c}\text { spin net space } \\
\text { SN } 09 \\
\left(10^{-36} \mathrm{sec}\right)\end{array}$ & $\begin{array}{c}\text { spin net space } \\
\text { SN } 11 \\
\left(10^{-34} \mathrm{sec}\right)\end{array}$ & $\begin{array}{l}\text { spin net space } \\
\text { SN } 13 \\
\left(10^{-32} \mathrm{sec}\right)\end{array}$ \\
\hline $\begin{array}{l}\text { arche-state vector } \\
A S 01\left(10^{-44} \mathrm{sec}\right)\end{array}$ & $\begin{array}{c}\text { clus infl config } \\
1\end{array}$ & & & & \\
\hline $\begin{array}{l}\text { arche-state vector } \\
A S 03\left(10^{-42} \mathrm{sec}\right)\end{array}$ & $\begin{array}{c}\text { clus infl config } \\
229.923 \times 10 \\
33\end{array}$ & & & & \\
\hline $\begin{array}{l}\text { arche-state vector } \\
A S 05\left(10^{-40} \mathrm{sec}\right)\end{array}$ & $\begin{array}{c}\text { clus infl config } \\
234.799 \times 10 \\
42\end{array}$ & $\begin{array}{l}\text { clus infl config } \\
236.890 \times 10^{39}\end{array}$ & & & \\
\hline $\begin{array}{l}\text { arche-state vector } \\
A S 06\left(10^{-39} \mathrm{sec}\right)\end{array}$ & & $\begin{array}{l}\text { clus infl config } \\
236.954 \times 10^{42}\end{array}$ & & & \\
\hline $\begin{array}{l}\text { arche-state vector } \\
A S 07\left(10^{-38} \mathrm{~cm}\right)\end{array}$ & & $\begin{array}{l}\text { clus infl config } \\
236.960 \times 10^{45}\end{array}$ & & & \\
\hline $\begin{array}{l}\text { arche-state vector } \\
A S 08\left(10^{-37} \mathrm{sec}\right)\end{array}$ & & & $\begin{array}{l}\text { clus infl config } \\
236.961 \times 10^{48}\end{array}$ & & \\
\hline $\begin{array}{l}\text { arche-state vector } \\
A S 09\left(10^{-36} \mathrm{sec}\right)\end{array}$ & & & $\begin{array}{l}\text { clus infl config } \\
236.962 \times 10^{50}\end{array}$ & & \\
\hline $\begin{array}{l}\text { arche-state vector } \\
A S 10\left(10^{-35} \mathrm{sec}\right)\end{array}$ & & & $\begin{array}{l}\text { clus infl config } \\
236.963 \times 10^{67}\end{array}$ & $\begin{array}{l}\text { clus infl config } \\
236.963 \times 10^{72}\end{array}$ & \\
\hline $\begin{array}{l}\text { arche-state vector } \\
A S 11\left(10^{-34} \mathrm{sec}\right)\end{array}$ & & & & $\begin{array}{l}\text { clus infl config } \\
236.964 \times 10^{93}\end{array}$ & \\
\hline $\begin{array}{l}\text { arche-state vector } \\
A S 12\left(10^{-33} \mathrm{sec}\right)\end{array}$ & & & & $\begin{array}{l}\text { clus infl config } \\
236.965 \times 10^{110}\end{array}$ & $\begin{array}{l}\text { clus infl config } \\
236.965 \times 10^{114}\end{array}$ \\
\hline $\begin{array}{l}\text { arche-state vector } \\
A S 13\left(10^{-32} \mathrm{sec}\right)\end{array}$ & & & & & $\begin{array}{l}\text { clus infl config } \\
236.966 \times 10^{135}\end{array}$ \\
\hline
\end{tabular}

Table 1 Cluster-inflating Configurations in Different Spin network spaces and Arche-State Vectors

$$
=0.808 \times 10^{-33} \mathrm{~cm}+9 \times 10^{-23} \mathrm{~cm} \approx 9.00000000808 \times 10^{-23} \mathrm{~cm}
$$




$$
\begin{aligned}
R & =9.000000000808 \times 10^{-23} \mathrm{~cm}+c^{2} \times 10^{-42} \mathrm{~s} \\
& =9.000000000808 \times 10^{-23} \mathrm{~cm}+\left(3 \times 10^{10} \mathrm{~cm} \cdot \mathrm{s}^{-1}\right)^{2} \times 10^{-42} \mathrm{~s} \\
& =9.000000000808 \times 10^{-23} \mathrm{~cm}+9 \times 10^{-22} \mathrm{~cm} \approx 9.900000000808 \times 10^{-22} \mathrm{~cm} \\
R & =9.900000000808 \times 10^{-22} \mathrm{~cm}+c^{2} \times 10^{-41} \mathrm{~s} \\
& =9.900000000808 \times 10^{-22} \mathrm{~cm}+\left(3 \times 10^{10} \mathrm{~cm} \cdot \mathrm{s}^{-1}\right)^{2} \times 10^{-41} \mathrm{~s} \\
& =9.900000000808 \times 10^{-22} \mathrm{~cm}+9 \times 10^{-21} \mathrm{~cm} \approx 9.990000000808 \times 10^{-21} \mathrm{~cm}
\end{aligned}
$$

\begin{tabular}{|c|c|c|c|c|c|}
\hline & $\begin{array}{l}\text { action mode } \\
\text { vector } F M 01 \\
\left(10^{-44} \mathrm{sec}\right)\end{array}$ & $\begin{array}{c}\text { action mode } \\
\text { vector } F M 06 \\
\left(10^{-39} \mathrm{sec}\right)\end{array}$ & $\begin{array}{l}\text { action mode } \\
\text { vector } F M 09 \\
\left(10^{-36} \mathrm{sec}\right)\end{array}$ & $\begin{array}{l}\text { action mode } \\
\text { vector } F M 11 \\
\left(10^{-34} \mathrm{sec}\right)\end{array}$ & $\begin{array}{c}\text { action mode } \\
\text { vector } F M 13 \\
\left(10^{-32} \mathrm{sec}\right)\end{array}$ \\
\hline $\begin{array}{c}\text { spin net space } \\
S N 01\left(10^{-44} \mathrm{sec}\right)\end{array}$ & $\begin{array}{c}\text { clus infl config } \\
1\end{array}$ & & & & \\
\hline $\begin{array}{c}\text { spin net space } \\
S N 03\left(10^{-42} \mathrm{sec}\right)\end{array}$ & $\begin{array}{c}\text { clus infl config } \\
229.923 \times 10 \\
33\end{array}$ & & & & \\
\hline $\begin{array}{c}\text { spin net space } \\
S N 05\left(10^{-40} \mathrm{sec}\right)\end{array}$ & $\begin{array}{c}\text { clus infl config } \\
234.799 \times 10 \\
42\end{array}$ & $\begin{array}{l}\text { clus infl config } \\
236.890 \times 10^{39}\end{array}$ & & & \\
\hline $\begin{array}{c}\text { spin net space } \\
S N 06\left(10^{-39} \mathrm{sec}\right)\end{array}$ & & $\begin{array}{l}\text { clus infl config } \\
236.954 \times 10^{42}\end{array}$ & & & \\
\hline $\begin{array}{c}\text { spin net space } \\
S N 07\left(10^{-38} \mathrm{~cm}\right)\end{array}$ & & $\begin{array}{l}\text { clus infl config } \\
236.960 \times 10^{45}\end{array}$ & & & \\
\hline $\begin{array}{c}\text { spin net space } \\
S N 08\left(10^{-37} \mathrm{sec}\right)\end{array}$ & & & $\begin{array}{l}\text { clus infl config } \\
236.961 \times 10^{48}\end{array}$ & & \\
\hline $\begin{array}{c}\text { spin net space } \\
S N 09\left(10^{-36} \mathrm{sec}\right)\end{array}$ & & & $\begin{array}{l}\text { clus infl config } \\
236.962 \times 10^{50}\end{array}$ & & \\
\hline $\begin{array}{c}\text { spin net space } \\
S N 10\left(10^{-35} \mathrm{sec}\right)\end{array}$ & & & $\begin{array}{l}\text { clus infl config } \\
236.963 \times 10^{67}\end{array}$ & $\begin{array}{l}\text { clus infl config } \\
236.963 \times 10^{72}\end{array}$ & \\
\hline $\begin{array}{c}\text { spin net space } \\
S N 11\left(10^{-34} \mathrm{sec}\right)\end{array}$ & & & & $\begin{array}{l}\text { clus infl config } \\
236.964 \times 10^{93}\end{array}$ & \\
\hline $\begin{array}{c}\text { spin net space } \\
S N 12\left(10^{-33} \mathrm{sec}\right)\end{array}$ & & & & $\begin{array}{l}\text { clus infl config } \\
236.965 \times 10^{110}\end{array}$ & $\begin{array}{l}\text { clus infl config } \\
236.965 \times 10^{114}\end{array}$ \\
\hline $\begin{array}{c}\text { spin net space } \\
S N 13\left(10^{-32} \mathrm{sec}\right)\end{array}$ & & & & & $\begin{array}{l}\text { clus infl config } \\
236.966 \times 10^{135}\end{array}$ \\
\hline
\end{tabular}

Table 2 Cluster-inflating Configurations in Action Mode Vector and Different Spin network spaces 
Tble 3 (Li Zongcheng, 2019) shows the spin network space at the different times of the creation of the universe. Table 4 (Li Zongcheng, 2019) shows the state vectors of arche-pulsator at the different times of the creation of the universe.

$$
\begin{aligned}
R & =9.990000000808 \times 10^{-21} \mathrm{~cm}+c^{2} \times 10^{-40} \mathrm{~s} \\
& =9.990000000808 \times 10^{-21} \mathrm{~cm}+\left(3 \times 10^{10} \mathrm{~cm} \cdot \mathrm{s}^{-1}\right)^{2} \times 10^{-40} \mathrm{~s} \\
& =9.900000000808 \times 10^{-22} \mathrm{~cm}+9 \times 10^{-20} \mathrm{~cm} \approx 9.999000000808 \times 10^{-20} \mathrm{~cm} \\
R & =9.999000000808 \times 10^{-20} \mathrm{~cm}+c^{2} \times 10^{-39} \mathrm{~s} \approx 9.999900000808 \times 10^{-19} \mathrm{~cm} \\
R & =9.999900000808 \times 10^{-19} \mathrm{~cm}+c^{2} \times 10^{-38} \mathrm{~s} \approx 9.999990000808 \times 10^{-18} \mathrm{~cm} \\
R & =9.999990000808 \times 10^{-18} \mathrm{~cm}+c^{2} \times 10^{-37} \mathrm{~s} \approx 9.999999000808 \times 10^{-17} \mathrm{~cm} \\
R & =9.999999000808 \times 10^{-17} \mathrm{~cm}+c^{2} \times 10^{-36} \mathrm{~s} \approx 9.999999900808 \times 10^{-16} \mathrm{~cm} \\
& \begin{array}{r}
\rho_{u-p h y} \\
=
\end{array} \\
& =4.7488 \times 10^{66} \mathrm{GeV} \times \frac{1}{4} \pi\left(9.9999 \times 10^{-18} \mathrm{~cm}\right)^{3}
\end{aligned}
$$

\begin{tabular}{|c|c|c|c|c|c|c|}
\hline & $\begin{array}{l}\text { spin quantum } \\
\text { number } \\
\text { S } 01\end{array}$ & $\begin{array}{l}\text { spin angular } \\
\text { momentum } \\
\quad S 02\end{array}$ & $\begin{array}{c}\text { spatial } \\
\text { dimension } \\
\text { S03 }\end{array}$ & $\begin{array}{c}\text { radius } \\
\text { of action } \\
\text { S } 04\end{array}$ & $\begin{array}{l}\text { number of } \\
\text { net nodes } \\
\text { S } 05\end{array}$ & $\begin{array}{c}\text { concentration } \\
\text { ratio } \\
S 06\end{array}$ \\
\hline $\begin{array}{c}\text { spin net space } \\
S N 01\left(10^{-44} \mathrm{sec}\right)\end{array}$ & $\begin{array}{c}0 \\
\text { Higgs field }\end{array}$ & & $\begin{array}{l}\geq 10 \mathrm{~d} \\
\text { contract }\end{array}$ & $\begin{array}{c}0.5 \times 1.616 \times \\
10^{-33} \mathrm{~cm}\end{array}$ & 1 & 1 \\
\hline $\begin{array}{c}\text { spin net space } \\
S N 03\left(10^{-42} \mathrm{sec}\right)\end{array}$ & $\begin{array}{cc}0 & \text { Higgs } \\
2 & \text { graviton }\end{array}$ & & $\begin{array}{c}\quad \leq 10 \mathrm{~d} \\
\text { extended } 4 \mathrm{~d}\end{array}$ & $\begin{array}{c}0.5 \times 9.900000 \\
\times 10^{-22} \mathrm{~cm}\end{array}$ & $\begin{array}{l}229.923 \\
\times 10^{33}\end{array}$ & $1.5924 \times 10^{-4}$ \\
\hline $\begin{array}{c}\text { spin net space } \\
\text { SN } 05\left(10^{-40} \mathrm{sec}\right)\end{array}$ & $\begin{array}{cc}2 & \text { graviton } \\
1 & \text { photon } \\
\end{array}$ & $\begin{array}{l}h / 2 \pi \\
\text { photon }\end{array}$ & $\begin{array}{c}\quad \leq 10 \mathrm{~d} \\
\text { extended } 4 \mathrm{~d}\end{array}$ & $\begin{array}{c}0.5 \times 9.999000 \\
\times 10^{-20} \mathrm{~cm} \\
\end{array}$ & $\begin{array}{r}236.890 \\
\times 10^{39} \\
\end{array}$ & $\begin{array}{c}0.0000000149 \\
\times 10^{-4}\end{array}$ \\
\hline $\begin{array}{c}\text { spin net space } \\
\text { SN } 06\left(10^{-39} \mathrm{sec}\right)\end{array}$ & $\begin{array}{cc}2 & \text { graviton } \\
1 & \text { photon }\end{array}$ & $\begin{array}{l}h / 2 \pi \\
\text { photon }\end{array}$ & $\begin{array}{c}\quad \leq 10 \mathrm{~d} \\
\text { extended } 4 \mathrm{~d}\end{array}$ & $\begin{array}{c}0.5 \times 9.999900 \\
\times 10^{-19} \mathrm{~cm}\end{array}$ & $\begin{array}{l}236.954 \\
\times 10^{42}\end{array}$ & $\begin{array}{l}1.44209 \\
\times 10^{-6}\end{array}$ \\
\hline $\begin{array}{c}\text { spin net space } \\
S N 07\left(10^{-38} \mathrm{sec}\right)\end{array}$ & $\begin{array}{cc}2 & \text { graviton } \\
1 & \text { photon } \\
\end{array}$ & $\begin{array}{l}h / 2 \pi \\
\text { photon }\end{array}$ & $\begin{array}{l}\quad \leq 10 \mathrm{~d} \\
\text { extended } 4 \mathrm{~d}\end{array}$ & $\begin{array}{c}0.5 \times 9.999990 \\
\times 10^{-18} \mathrm{~cm}\end{array}$ & $\begin{array}{l}236.960 \\
\times 10^{45}\end{array}$ & $\begin{array}{r}0.88846 \\
\times 10^{-7}\end{array}$ \\
\hline spin net space & 2 graviton & $h / 2 \pi$ & $\leq 10 \mathrm{~d}$ & $0.5 \times 9.999999$ & 236.961 & 0.67682 \\
\hline
\end{tabular}

Table 3 Spin Network Space at Different Moment of Creation of Universe 


\begin{tabular}{|c|c|c|c|c|c|c|}
\hline$S N 08\left(10^{-37} \mathrm{sec}\right)$ & 1 photon & photon & extended $4 d$ & $\times 10^{-17} \mathrm{~cm}$ & $\times 10^{48}$ & $\times 10^{-8}$ \\
\hline spin net space & 2 graviton & $h / 2 \pi$ & $\leq 10 \mathrm{~d}$ & $0.5 \times 9.999999$ & 236.962 & 0.20825 \\
\hline$S N 09\left(10^{-36} \mathrm{sec}\right)$ & 1 photon & photon & extended 4d & $\times 10^{-16} \mathrm{~cm}$ & $\times 10^{50}$ & $\times 10^{-10}$ \\
\hline spin net space & 2 graviton & & $\leq 10 \mathrm{~d}$ & $1.5 \times 0.5 \times 9.9999$ & 236.963 & 0.12566 \\
\hline$S N 10\left(10^{-35} \mathrm{sec}\right)$ & $1 / 2 \quad$ v-quark & $\overline{2 \pi}\left(\frac{-\sigma}{2}\right)$ & extended $4 d$ & $\times 10^{-9} \mathrm{~cm}$ & $\times 10^{72}$ & $\times 10^{-16}$ \\
\hline spin net space & 2 graviton & $\hbar,(\hbar \hat{\tilde{\sigma}})^{2}$ & $\leq 10 \mathrm{~d}$ & $(1.5)^{2} \times 0.5 \times$ & 236.964 & 1.159707 \\
\hline$S N 11\left(10^{-34} \mathrm{sec}\right)$ & $1 / 2 \quad$ v-quark & $\overline{2 \pi}\left(\overline{2}^{\sigma}\right)$ & extended 4d & $9.999 \times 10^{-2} \mathrm{~cm}$ & $\times 10^{93}$ & $\times 10^{-37}$ \\
\hline spin net space & 2 graviton & $\underline{\hbar},\left(\frac{\hbar}{\hat{\sigma}}\right)^{2}$ & $\leq 10 \mathrm{~d}$ & $(1.5)^{3} \times 0.5 \times$ & 236.965 & 0.67546 \\
\hline$S N 12\left(10^{-33} \mathrm{sec}\right)$ & $1 / 2 \quad v$-quark & $\overline{2 \pi}(\overline{2} \sigma)$ & extended $4 d$ & $9.999 \times 10^{5} \mathrm{~cm}$ & $\times 10^{114}$ & $\times 10^{-46}$ \\
\hline spin net space & 2 graviton & $\left.(\hbar)^{2}\right)^{2}$ & $\leq 10 \mathrm{~d}$ & $(1.5)^{4} \times 0.5 \times$ & 236.966 & 1.46779 \\
\hline$S N 13\left(10^{-32} \mathrm{sec}\right)$ & $1 / 2 \quad$ v-quark & $\overline{2 \pi}\left(\frac{-\sigma}{2}\right)$ & extended $4 d$ & $9.999 \times 10^{12} \mathrm{~cm}$ & $\times 10^{135}$ & $\times 10^{-61}$ \\
\hline
\end{tabular}

Table 4 State Vector of Arche-Pulsator at Different Moment of Creation of Universe

\begin{tabular}{|c|c|c|c|c|c|c|}
\hline & $\begin{array}{l}\text { dimension } \\
\quad S 01\end{array}$ & $\begin{array}{l}\text { Pulsation } \\
\text { frequency } \\
\quad S 02\end{array}$ & $\begin{array}{l}\text { basic form } \\
\qquad S 03\end{array}$ & $\begin{array}{l}\text { Lyapunov index } \\
\qquad S 04\end{array}$ & $\begin{array}{c}\text { total } \\
\text { number } \\
S 05\end{array}$ & $\begin{array}{c}\text { concentration } \\
\text { ratio } \\
S 06\end{array}$ \\
\hline $\begin{array}{c}\text { state vector } \\
S V 01\left(10^{-44} \mathrm{sec}\right)\end{array}$ & $\begin{array}{r}1.616 \\
\times 10^{-33} \mathrm{~cm}\end{array}$ & & & & 1 & 1 \\
\hline $\begin{array}{c}\text { state vector } \\
S V 03\left(10^{-42} \mathrm{sec}\right)\end{array}$ & $\begin{array}{r}1.616 \\
\times 10^{-33} \mathrm{~cm}\end{array}$ & $\begin{array}{r}3.84913 \\
\times 10^{-42} \mathrm{sec}\end{array}$ & $\begin{array}{l}\text { chaotic } \\
\text { pulsator }\end{array}$ & $\begin{array}{c}\text { Lyapunov index } \lambda \\
\text { undetermined }\end{array}$ & $\begin{array}{r}229.923 \\
\times 10^{33}\end{array}$ & $1.5924 \times 10^{-4}$ \\
\hline $\begin{array}{c}\text { state vector } \\
S V 05\left(10^{-40} \mathrm{sec}\right)\end{array}$ & $\begin{array}{r}1.616 \\
\times 10^{-33} \mathrm{~cm} \\
\end{array}$ & $\begin{array}{r}7.29824 \\
\times 10^{-40} \mathrm{sec} \\
\end{array}$ & $\begin{array}{l}\text { chaotic } \\
\text { pulsator }\end{array}$ & $\begin{array}{c}\text { Lyapunov index } \lambda \\
\text { undetermined }\end{array}$ & $\begin{array}{r}236.890 \\
\times 10^{39} \\
\end{array}$ & $\begin{array}{c}0.0000000149 \\
\times 10^{-4} \\
\end{array}$ \\
\hline $\begin{array}{c}\text { state vector } \\
S V 06\left(10^{-39} \mathrm{sec}\right)\end{array}$ & $\begin{array}{r}1.616 \\
\times 10^{-33} \mathrm{~cm} \\
\end{array}$ & $\begin{array}{r}4.28722 \\
\times 10^{-39} \mathrm{sec}\end{array}$ & $\begin{array}{l}\text { chaotic } \\
\text { pulsator }\end{array}$ & $\begin{array}{c}\text { Lyapunov index } \lambda \\
\text { undetermined }\end{array}$ & $\begin{array}{r}236.954 \\
\times 10^{42}\end{array}$ & $\begin{array}{l}1.44209 \\
\times 10^{-6}\end{array}$ \\
\hline $\begin{array}{c}\text { state vector } \\
S V 07\left(10^{-38} \mathrm{sec}\right)\end{array}$ & $\begin{array}{r}1.616 \\
\times 10^{-33} \mathrm{~cm} \\
\end{array}$ & $\begin{array}{r}2.48927 \\
\times 10^{-38} \mathrm{sec} \\
\end{array}$ & $\begin{array}{l}\text { chaotic } \\
\text { pulsator }\end{array}$ & $\begin{array}{c}\text { Lyapunov index } \lambda \\
\text { undetermined }\end{array}$ & $\begin{array}{r}236.960 \\
\times 10^{45} \\
\end{array}$ & $\begin{array}{l}0.88846 \\
\times 10^{-7} \\
\end{array}$ \\
\hline $\begin{array}{c}\text { state vector } \\
S V 08\left(10^{-37} \mathrm{sec}\right)\end{array}$ & $\begin{array}{r}1.616 \\
\times 10^{-33} \mathrm{~cm} \\
\end{array}$ & $\begin{array}{r}8.28392 \\
\times 10^{-37} \mathrm{sec} \\
\end{array}$ & $\begin{array}{l}\text { chaotic } \\
\text { pulsator }\end{array}$ & $\begin{array}{c}\text { Lyapunov index } \lambda \\
\text { undetermined }\end{array}$ & $\begin{array}{r}236.961 \\
\times 10^{48} \\
\end{array}$ & $\begin{array}{c}0.67682 \\
\times 10^{-8} \\
\end{array}$ \\
\hline $\begin{array}{c}\text { state vector } \\
S V 09\left(10^{-36} \mathrm{sec}\right)\end{array}$ & $\begin{array}{r}1.616 \\
\times 10^{-33} \mathrm{~cm} \\
\end{array}$ & $\begin{array}{r}4.37710 \\
\times 10^{-36} \mathrm{sec} \\
\end{array}$ & $\begin{array}{l}\text { chaotic } \\
\text { pulsator }\end{array}$ & $\begin{array}{c}\text { Lyapunov index } \lambda \\
\text { undetermined }\end{array}$ & $\begin{array}{c}236.962 \\
\times 10^{50} \\
\end{array}$ & $\begin{array}{r}0.20825 \\
\times 10^{-10} \\
\end{array}$ \\
\hline $\begin{array}{c}\text { state vector } \\
S V 10\left(10^{-35} \mathrm{sec}\right)\end{array}$ & $\begin{array}{r}1.616 \\
\times 10^{-33} \mathrm{~cm} \\
\end{array}$ & $\begin{array}{r}5.43371 \\
\times 10^{-35} \mathrm{sec} \\
\end{array}$ & $\begin{array}{l}\text { quasi-sph } \\
\text { pulsator }\end{array}$ & $\begin{array}{c}\text { Lyapunov index } \lambda \\
\text { undetermined }\end{array}$ & $\begin{array}{r}236.963 \\
\times 10^{72} \\
\end{array}$ & $\begin{array}{r}0.12566 \\
\times 10^{-16} \\
\end{array}$ \\
\hline $\begin{array}{c}\text { state vector } \\
S V 11\left(10^{-34} \mathrm{sec}\right)\end{array}$ & $\begin{array}{r}1.616 \\
\times 10^{-33} \mathrm{~cm} \\
\end{array}$ & $\begin{array}{r}6.79272 \\
\times 10^{-34} \mathrm{sec} \\
\end{array}$ & $\begin{array}{l}\text { quasi-sph } \\
\text { pulsator }\end{array}$ & $\begin{array}{c}\text { Lyapunov index } \lambda \\
\text { undetermined }\end{array}$ & $\begin{array}{r}236.964 \\
\times 10^{93} \\
\end{array}$ & $\begin{array}{l}1.159707 \\
\times 10^{-37} \\
\end{array}$ \\
\hline $\begin{array}{c}\text { state vector } \\
S V 12\left(10^{-33} \mathrm{sec}\right)\end{array}$ & $\begin{array}{r}1.616 \\
\times 10^{-33} \mathrm{~cm} \\
\end{array}$ & $\begin{array}{r}5.39116 \\
\times 10^{-33} \mathrm{sec} \\
\end{array}$ & $\begin{array}{l}\text { quasi-sph } \\
\text { pulsator }\end{array}$ & $\begin{array}{c}\text { Lyapunov index } \lambda \\
\text { undetermined }\end{array}$ & $\begin{array}{r}236.965 \\
\times 10^{114} \\
\end{array}$ & $\begin{array}{r}0.67546 \\
\times 10^{-46} \\
\end{array}$ \\
\hline $\begin{array}{c}\text { state vector } \\
S V 13\left(10^{-32} \mathrm{sec}\right)\end{array}$ & $\begin{array}{r}1.616 \\
\times 10^{-33} \mathrm{~cm}\end{array}$ & $\begin{array}{r}6.20811 \\
\times 10^{-32} \mathrm{sec}\end{array}$ & $\begin{array}{l}\text { quasi-sph } \\
\text { pulsator }\end{array}$ & $\begin{array}{c}\text { Lyapunov index } \lambda \\
\text { undetermined }\end{array}$ & $\begin{array}{r}236.966 \\
\times 10^{135}\end{array}$ & $\begin{array}{r}1.46779 \\
\times 10^{-61}\end{array}$ \\
\hline
\end{tabular}




$$
V_{u-p h y}=\frac{4}{3} \pi R^{3}=\frac{4}{3} \times 3.14 \times\left(9.9999 \times 10^{-18} \mathrm{~cm}\right)^{3}=4.186541 \times 10^{-51} \mathrm{~cm}^{3}
$$

When the universe was at $t=10^{-36} \mathrm{~s}$, the temperature $T=1.22 \times 10^{28} \mathrm{~K}$ of the universe, the energy $E_{u-p h y}=1.042603 \times 10^{72} \mathrm{GeV}$ of the universe, $m_{u-p h y}=0, R=9.9999 \times 10^{-16} \mathrm{~cm}$, 密度 $\rho_{u-p h y}=$ $4.071902 \times 10^{80} \mathrm{~kg} \cdot \mathrm{cm}^{-3}$, the volume $V_{u-p h y}=4.18654 \times 10^{-45} \mathrm{~cm}^{3}$ of the universe

$$
\begin{aligned}
& E_{u-p h y}= 0.55 \times 10^{18} \mathrm{GeV} \times\left(\frac{4}{3} \pi R^{3} / \frac{4}{3} \pi r_{\text {planck }}^{3}\right) \\
&= 0.55 \times 10^{18} \mathrm{GeV} \times\left[\left(9.9999 \times 10^{-16} \mathrm{~cm}\right)^{3} /\left(\frac{1}{2} \times 1.616 \times 10^{-33} \mathrm{~cm}\right)^{3}\right] \\
&= 0.55 \times 10^{18} \mathrm{GeV} \times \frac{999.97 \times 10^{-48} \mathrm{~cm}}{0.52751 \times 10^{-99} \mathrm{~cm}}=1.042603 \times 10^{72} \mathrm{GeV} \\
& R \approx 9.999999000808 \times 10^{-17} \mathrm{~cm}+\left(3 \times 10^{10} \mathrm{~cm} \cdot \mathrm{s}^{-1}\right)^{2} \times 10^{-36} \mathrm{~s} \\
&=9.999999000808 \times 10^{-17} \mathrm{~cm}+9 \times 10^{-16} \mathrm{~cm} \approx 9.999999900808 \times 10^{-16} \mathrm{~cm} \\
& \rho_{u-p h y}=1.042603 \times 10^{72} \mathrm{GeV} \times \frac{1}{\frac{4}{3} \pi\left(9.9999 \times 10^{-16} \mathrm{~cm}\right)^{3}} \\
&=1.67129 \times 10^{34} \mathrm{~J} \times \frac{1}{4186.541 \times 10^{-48} \mathrm{~cm}^{3}} \\
&=1.70472 \times 10^{36} \mathrm{~kg} \times \frac{1}{4186.541 \times 10^{-48} \mathrm{~cm}^{3}}=4.071902 \times 10^{80} \mathrm{~kg} \cdot \mathrm{cm}^{-3} \\
& V_{u-p h y}= \frac{4}{3} \pi R^{3}=\frac{4}{3} \times 3.14 \times\left(9.9999 \times 10^{-16} \mathrm{~cm}^{3}=4.18654 \times 10^{-45} \mathrm{~cm}^{3}\right.
\end{aligned}
$$

\section{Operator Distribution Function of Interaction Mode}

What we have obtained above is the equation of motion describing quantum gravi-operators (mode vectors or density matrices). Unless the system itself has only finite mechanical states (operators correspond to finite dimensional matrices), it will be very difficult to obtain quantum effects directly and correctly (for example, considering the vibration of zeros, the non-commutative properties of operators, etc.). Next, we will establish a Fokker-Planck equation which can be transformed from the operator equation to the $C$-number function (operator distribution function), so that we can solve many problems by using the traditional method of solving partial differential equations.

In the interaction system $S$, a complete set $a_{1}, a_{2}, \cdots, a_{N}$ of operators which obeys certain commutative or anti-commutative relations is chosen. For example, for a commutative quantum gravi-system, $a$ and $a^{\dagger}$ are corresponding annihilation and generation operators respectively, $\left[a, a^{\dagger}\right]=$ 1, then $a_{1}=a, a_{2}=a^{\dagger}$. Any operator $Q$ of $S$ can be expanded by $\left\{a_{i}\right\}$ and its product. Because it is not commutative, we have to arrange a sequence (for example, the order is $a_{1}, a_{2}, \cdots, a_{N}$ ) of $\left\{a_{i}\right\}$ 
products beforehand, which is called $N$ order. So, the expansion of $Q$ is

$$
Q=\sum_{\left\{r_{i}\right\}} q_{N}\left(r_{1}, r_{2}, \cdots, r_{N}\right) a_{1}^{r_{1}} a_{2}^{r_{2}} \cdots a_{N}^{r_{N}}
$$

here $\sum_{\left\{r_{i}\right\}}=\sum_{r_{1}} \sum_{r_{2}} \cdots \sum_{r_{N}}$. Introducing $C$-Number Function

$$
Q_{N}\left(\alpha_{1}, \alpha_{2} \cdots, \alpha_{N}\right)=\sum_{\left\{r_{i}\right\}} q_{N}\left(r_{1}, r_{2}, \cdots, r_{N}\right) a_{1}^{r_{1}} a_{2}^{r_{2}} \cdots a_{N}^{r_{N}}
$$

$\alpha_{1}, \alpha_{2}, \cdots, \alpha_{N}$ is $N$ independent variables, then we establish a one-to-one correspondence between the ordering operator function $Q$ and the $C$ number function $Q_{N}$. We can use $Q_{N}$ to express $Q$ :

$$
Q=\int d \alpha Q_{N}(\alpha) \delta\left(\alpha_{1}-a_{1}\right) \delta\left(\alpha_{2}-a_{2}\right) \cdots \delta\left(\alpha_{N}-a_{N}\right)
$$

here $d \alpha=d \alpha_{1} \cdots d \alpha_{N}$. In formula ( 130 ), special attention should be paid to the order of $\delta$ functions. If $a_{i}$ is a Hermitian operator and $\alpha_{i}$ is a real variable, the operator's $\delta$ function can be expressed as

$$
\delta\left(\alpha_{i}-a_{i}\right)=\frac{1}{2 \pi} \int_{\infty}^{-\infty} d b_{i} e^{-i b_{i}\left(\alpha_{i}-a_{i}\right)}
$$

If $a_{i}$ is not Hermitian, then $\alpha_{i}$ is a complex variable and there is

$$
\delta\left(\alpha_{i}^{*}-a_{i}^{\dagger}\right) \delta\left(\alpha_{i}-a_{i}\right)=\frac{1}{\pi^{2}} \int_{\infty}^{-\infty} d^{2} b_{i} e^{-i b_{i}^{*}\left(\alpha_{i}^{*}-a_{i}^{*}\right)} e^{-i b_{i}\left(\alpha_{i}-a_{i}\right)}
$$

here $d^{2} b_{i}=d \operatorname{Re}\left(b_{i}\right) d \operatorname{Im}\left(b_{i}\right)$; formula ( 132$)$ is the integral of the whole complex plane of $b_{i}$.

If the density matrix of the interaction system $S$ is $\rho_{S}(t)$, then there is

$$
\begin{aligned}
\operatorname{Tr} Q \rho_{S}(t) & =\int d \alpha Q_{N}(\alpha) \operatorname{Tr}_{S} \delta\left(\alpha_{1}-a_{1}\right) \delta\left(\alpha_{2}-a_{2}\right) \cdots \delta\left(\alpha_{N}-a_{N}\right) \rho_{S}(t) \\
& =\int d \alpha Q_{N}(\alpha) P(\alpha, t)
\end{aligned}
$$

here

$$
P(\alpha, t)=\operatorname{Tr}_{S} \delta\left(\alpha_{1}-a_{1}\right) \delta\left(\alpha_{2}-a_{2}\right) \cdots \delta\left(\alpha_{N}-a_{N}\right) \rho_{S}(t)
$$

is called the distribution function of density matrix $\rho_{S}(t)$, or operator distribution function for short. Obviously, if $P(\alpha, t)$ is obtained, we can also get the average value of all mechanical quantities, thus we can know the complete dynamic behavior of the system. The operator distribution function is equivalent to the density matrix or a complete set of operators (Heisenberg image) to describe the motion of the system.

In relativistic quantum mechanics and subsequent quantum field theory, the idea of "ensemble" has been included ${ }^{[51][102]-[104]}$. This shows that ensemble interpretation of quantum mechanics has a broader application than the Copenhagen interpretation of quantum mechanics. In recent years, the quantum mechanics of dissipative systems has made some progress ${ }^{[51][104]-[106]}$, where one of the important methods ( Feynman and Vernon, Leggett ) is to represent the environment with a large number of harmonic oscillator groups, and the harmonic oscillator with the system interact each other; the path integral of system-environment is written, and the environment coordinate is integrated, in order to obtain the effective theory of dissipative effect of system under the action of environment.

For the high-energy cosmic ray, astrophysics circles in the discovery of the positron at the same time, also found the electromagnetic cascade shower, which is the process produces of a large number 
of positive and negative secondary electrons and photons from a high-energy electron or photon in the air or other material, after repeated electromagnetic action. Afterwards the phenomenon of extensive air shower is discorerd, it is the nuclear cascade process for the hadron of cosmic ray with the energy higher than $10^{14} \mathrm{eV}$ in interaction with the nucleus at the top of atmosphere to produce a large number of secondary hadron which continue to act. In this process, $2 \gamma$ photon from the decaying of $\pi^{0}$ meson, then quickly form electromagnetic cascade shower. The secondary cosmic rays near the sea level can be divided into two components: hard and soft. The soft component is almost all absorbed after $10 \mathrm{~cm}$ of lead, which is mainly electrons and $\gamma$ photons; the hard component can penetrate the very thick material layer, which is mainly the muon. The $\pi$ meson, $K$ meson, $\Lambda^{0}, \Sigma^{ \pm, 0}$ and $\Xi^{-}$hyperon, etc. have been found in cosmic rays. The nuclear effect of cosmic rays in the chamber and nuclear emulsion also gives good results, the phenomenon of injection has been found.

The Fourier transform of operator distribution function is often used:

$$
G(\eta, t)=\int d \alpha e^{i\left(\alpha_{1} \eta_{1}+\alpha_{2} \eta_{2}+\cdots+\alpha_{N} \eta_{N}\right)} P(\alpha, t)=\operatorname{Tr}_{S} e^{i \eta_{1} a_{1}} e^{i \eta_{2} a_{2}} \cdots e^{i \eta_{N} a_{N}} \rho_{S}(t)
$$

Since $G$ is the average value of the operator $\prod_{i} e^{i \eta_{i} a_{i}}$, we call it a generating function (in probability theory, it is called a characteristic function). Similarly, the inverse Fourier transform of $Q_{N}(\alpha)$ is introduced:

$$
\tilde{Q}_{N}(\eta)=\left(\frac{1}{2 \pi}\right)^{2} \int d \alpha e^{i\left(\alpha_{1} \eta_{1}+\alpha_{2} \eta_{2}+\cdots+\alpha_{N} \eta_{N}\right)} Q_{N}(\alpha)
$$

then

$$
\begin{gathered}
\operatorname{Tr} Q \rho_{S}(t)=\int d \eta \tilde{Q}_{N}(\alpha) G(\eta, t) \\
Q=\int d \eta \tilde{Q}_{N}(\eta) e^{i \eta_{1} a_{1}} e^{i \eta_{2} a_{2}} \cdots e^{i \eta_{N} a_{N}}
\end{gathered}
$$

We can see that if $P(\alpha, t)$ is regarded as the classical probability, the average value of the ordering operator is calculated as the classical average value; $\left\{\alpha_{i}\right\}$ can also be formally understood as the value of $\left\{a_{i}\right\}$. After introducing the generating function, the moments of $a$ can be written as

$$
\operatorname{Tr}_{1}^{r_{1}} a_{2}^{r_{2}} \cdots a_{N}^{r_{N}}=\left.\frac{\partial^{r_{1}+r_{2}+\cdots+r_{N}}}{\partial\left(i \eta_{1}\right)^{r_{1}} \partial\left(i \eta_{2}\right)^{r_{2}} \cdots \partial\left(i \eta_{N}\right)^{r_{N}}} G(\eta, t)\right|_{\left\{\eta_{i}=0\right\}}=\int d \alpha \alpha_{1}^{r_{1}} \alpha_{2}^{r_{2}} \cdots a_{N}^{r_{N}} P(\alpha, t)
$$

Obviously, $P(\alpha, t)$ is related to the order of selected operators, and it is not possible to restore $\rho_{S}$ ( $t$ ) directly from $P$. If we expand the $\rho_{S}(t)$ in the same order :

$$
\begin{aligned}
\rho_{S}(t) & =\sum_{\left\{r_{i}\right\}} \rho(r) a_{1}^{r_{1}} a_{2}^{r_{2}} \cdots a_{N}^{r_{N}} \\
& =\int d \alpha \rho_{S, N}(\alpha, t) \delta\left(\alpha_{1}-a_{1}\right) \delta\left(\alpha_{2}-a_{2}\right) \cdots \delta\left(\alpha_{N}-a_{N}\right)
\end{aligned}
$$

here

$$
\rho_{S, N}(\alpha, t)=\sum_{\left\{r_{i}\right\}} \rho(r) \alpha_{1}^{r_{1}} \alpha_{2}^{r_{2}} \cdots \alpha_{N}^{r_{N}}
$$


then

$$
P(\alpha, t)=\int d \beta \rho_{S, N}(\beta, t) K(\beta, \alpha)
$$

here

$$
K(\beta, \alpha)=\operatorname{Tr}\left\{\delta\left(\beta_{1}-a_{1}\right) \delta\left(\beta_{2}-a_{2}\right) \cdots \delta\left(\beta_{N}-a_{N}\right) \times \delta\left(\alpha_{1}-a_{1}\right) \delta\left(\alpha_{2}-a_{2}\right) \cdots \delta\left(\alpha_{N}-a_{N}\right)\right\}
$$

The elimination and generation operators $a$ and $a^{\dagger}$ of the interaction system satisfy the commutative relationship: $\left[a, a^{\dagger}\right]=1$. Any mechanical quantity can be written as a function of $a$ and $a^{\dagger}: f\left(a, a^{\dagger}\right)$. There should be two kinds of operator arrangement order: one is the normal order, $a^{\dagger}$ is on the left of $a$ and is recorded as $N$; the other is the anomalous order, $a$ is on the left of $a^{\dagger}$ and is recorded as $A$. Two sequential expressions of $f$ can be written by using the exchange relation :

$$
f\left(a, a^{\dagger}\right)=f_{N}\left(a, a^{\dagger}\right)=f_{A}\left(a, a^{\dagger}\right)
$$

and

$$
f=\int d^{2} \alpha f_{A}(\alpha) \delta(\alpha-a) \delta\left(\alpha^{*}-a^{\dagger}\right)=\int d^{2} \beta f_{N}(\beta) \delta\left(\beta^{*}-a^{\dagger}\right) \delta(\beta-a)
$$

here $f_{A}(\alpha)$ and $f_{N}(\beta)$ are $C$-number functions (It can be abbreviated as inverse order $C$-number function and positive order $C$-number function respectively) corresponding respectively to the inverse order operator function $f_{A}\left(a, a^{\dagger}\right)$ and the positive order operator function $f_{N}\left(a, a^{\dagger}\right)$.

The energy eigenvalue representation can be used to discuss the harmonic pulsator :

$$
a^{\dagger} a|n>=n| n>, \quad n=0,1,2, \cdots ;
$$

When discussing the coherence of light fields, it is more convenient to introduce coherent states: $a \mid \alpha>$ $=\alpha \mid a>$, and $\alpha$ takes the complex number.

$$
\begin{gathered}
\text { If you take }|\alpha\rangle=\sum_{n} C_{n}|n\rangle,\langle\alpha|=\sum_{n}\left\langle n \mid C_{n}^{*}\right\rangle \text { and }\langle\alpha \mid \alpha\rangle=1 \text {, it's easy to get } \\
C_{n}=e^{-\frac{1}{2}|\alpha|^{2}} \frac{\alpha^{n}}{\sqrt{n !}} .
\end{gathered}
$$

We note $|<n| \alpha>\left.\right|^{2}=e^{-|\alpha|^{2}}|\alpha|^{2 n} / n$ !, which is that the number of photons in coherent states satisfies

Poisson distribution. There is no orthogonality $|<\beta| \alpha>\left.\right|^{2}=e^{-|\alpha-\beta|^{2}} \neq 0$ between coherent states.

However, all coherent states form a complete group and there is $\int \frac{d^{2} \alpha}{\pi}|\alpha><\alpha|=1$.

In essence, they are a super-complete group. For regular ordering,

$$
f\left(a, a^{\dagger}\right)=\sum_{r_{1}, r_{2}} f_{N}\left(r_{1}, r_{2}\right) a^{\dagger r 1} a^{r 2}
$$

so

$$
<\alpha|f| \alpha>=\sum_{r_{1}, r_{2}} f_{N}\left(r_{1}, r_{2}\right) \alpha^{* r_{1}} \alpha^{r_{2}}=f_{N}(\alpha) a^{\dagger r 1} a^{r^{2}}
$$

Considering $\left[a, a^{\dagger}\right]=1, f_{N}(\alpha)$ can also be written as 


$$
f_{N}(\alpha)=f\left(\alpha+\frac{\partial}{\partial \alpha^{*}}, \alpha^{*}\right) \cdot 1
$$

Likewise, there should be

$$
f=\int \frac{d^{2} \alpha}{\pi}|\alpha><\alpha| f_{A}(\alpha), \quad f_{A}(\alpha)=f\left(\alpha, \alpha^{*}-\frac{\partial}{\partial \alpha}\right) \cdot 1
$$

\section{Motion Equation of ODF of Interaction Mode}

Time Partial Derivatives for Operator Distribution Function (ODF) $P(\alpha, t)$ is taken as follows

$$
\frac{\partial}{\partial t} P(\alpha, t)=\operatorname{Tr} \delta\left(\alpha_{1}-a_{1}\right) \delta\left(\alpha_{2}-a_{2}\right) \cdots \delta\left(\alpha_{N}-a_{N}\right) \frac{\partial}{\partial t} \rho_{S}
$$

The invariance of array rotation is used, then there is

$$
\begin{aligned}
\frac{\partial}{\partial t} P(\alpha, t)= & \operatorname{Tr}\left[i L_{S} \delta\left(\alpha_{1}-a_{1}\right) \delta\left(\alpha_{2}-a_{2}\right) \cdots \delta\left(\alpha_{N}-a_{N}\right)\right] \rho_{S} \\
& -\sum_{j, k} w_{k j}^{+} \operatorname{Tr}\left[\delta\left(\alpha_{1}-a_{1}\right) \delta\left(\alpha_{2}-a_{2}\right) \cdots \delta\left(\alpha_{N}-a_{N}\right), Q_{k}\right] Q_{j} \rho_{S} \\
& +\sum_{j, k} w_{j k}^{-} \operatorname{Tr} Q_{j}\left[\delta\left(\alpha_{1}-a_{1}\right) \delta\left(\alpha_{2}-a_{2}\right) \cdots \delta\left(\alpha_{N}-a_{N}\right), Q_{k}\right] \rho_{S}
\end{aligned}
$$

here $Q_{k}$ and $Q_{j}$ are functions of operator $a$. The representation $\delta\left(\alpha_{i}-a_{i}\right)=e^{-a_{i} \frac{\partial}{\partial \alpha_{i}}} \delta\left(\alpha_{i}\right)$ of translation group is used, then

$$
\frac{\partial}{\partial t} P(\alpha, t)=\operatorname{Tr} \rho_{S} L^{\prime}\left(\frac{\partial}{\partial \alpha}, a\right) \delta(\alpha)
$$

here

$$
\begin{gathered}
L^{\prime}\left(\frac{\partial}{\partial \alpha}, a\right)=i L_{S} e^{-a \frac{\partial}{\partial \alpha}}-\sum_{j, k} w_{j k}^{+}\left[e^{-a \frac{\partial}{\partial \alpha}}, Q_{k}\right] Q_{j}+\sum_{j, k} w_{k j}^{-} Q_{j}\left[e^{-a \frac{\partial}{\partial \alpha}}, Q_{k}\right] \\
e^{-a \frac{\partial}{\partial \alpha}}=e^{-a_{1} \frac{\partial}{\partial \alpha_{1}}} e^{-a_{2} \frac{\partial}{\partial \alpha_{2}}} \cdots e^{-a_{N} \frac{\partial}{\partial \alpha_{N}}} \\
\delta(\alpha)=\delta\left(\alpha_{1}\right) \delta\left(\alpha_{2}\right) \cdots \delta\left(\alpha_{N}\right)
\end{gathered}
$$

If the operators in $L^{\prime}$ are arranged in the selected $\operatorname{order}(N): L^{\prime}=L_{N}^{\prime}\left(\frac{\partial}{\partial \alpha}, a\right)$, then

$$
\frac{\partial}{\partial t} P(\alpha, t)=\operatorname{Tr} \rho_{S}(t) L_{N}^{\prime}\left(\frac{\partial}{\partial \alpha}, a\right) \delta(\alpha)
$$




$$
\begin{aligned}
& =\operatorname{Tr} \rho_{S}(t) \int d \beta L_{N}^{\prime}\left(\frac{\partial}{\partial \alpha}, \beta\right) \delta_{N}(\beta-a) \delta(\alpha) \\
& =\operatorname{Tr} \rho_{S}(t) \int d \beta \bar{L}_{N}\left(\frac{\partial}{\partial \alpha}, \beta\right) \delta_{N}(\beta-a) \delta(\alpha-\beta) \\
& =\operatorname{Tr} \rho_{S}(t) \bar{L}_{N}\left(\frac{\partial}{\partial \alpha}, \alpha\right) \delta_{N}(\alpha-a)
\end{aligned}
$$

here

$$
\begin{gathered}
\delta_{N}(\alpha-a)=\delta\left(\alpha_{1}-a_{1}\right) \delta\left(\alpha_{1}-a_{2}\right) \cdots \delta\left(\alpha_{N}-a_{N}\right) \\
\bar{L}_{N}\left(\frac{\partial}{\partial \alpha}, \beta\right) e^{-\beta \frac{\partial}{\partial \alpha}}=L_{N}^{\prime}\left(\frac{\partial}{\partial \alpha}, \beta\right)
\end{gathered}
$$

In ( 145 ), the operator $a$ is no longer included, so it can be moved out of the trace to obtain the equation of motion satisfied by $P(\alpha, t)$ :

$$
\frac{\partial}{\partial t} P(\alpha, t)=\bar{L}_{N}\left(\frac{\partial}{\partial \alpha}, \alpha\right) P(\alpha, t)
$$

It is noted that formula ( 146 ) contains all quantum effects, which are equivalent to Master equation satisfied by $\rho_{S}$. In many cases, derivatives higher than quadratic are either nonexistent or very small, formula ( 146 ) is reduced to the Fokker-Planck equation:

$$
\frac{\partial}{\partial t} P(\alpha, t) \approx-\sum_{j} \frac{\partial}{\partial \alpha_{j}}\left(A_{j}(\alpha) P\right)+\sum_{j, k} \frac{\partial^{2}}{\partial \alpha_{j} \partial \alpha_{k}}\left(D_{j k}(\alpha) P\right)
$$

In the specific derivation, it is more complicated to get $L^{\prime}{ }_{N}$ from $L^{\prime}$. Taking the two-level system as an example, the specific calculation steps are explained. Taking

$$
a_{1} \rightarrow \sigma_{+}=\left(\begin{array}{ll}
0 & 1 \\
0 & 0
\end{array}\right), \quad a_{2} \rightarrow \sigma_{-}=\left(\begin{array}{ll}
0 & 0 \\
1 & 0
\end{array}\right), \quad a_{3} \rightarrow \sigma_{3}=\left(\begin{array}{cc}
1 & 0 \\
0 & -1
\end{array}\right)
$$

then the Hamiltonian of the system is

$$
H_{S}=\frac{\varepsilon}{2} \sigma_{3}, \text { is the energy of the system, }
$$

and the distribution function of density matrix $\rho_{S}(t)$ is

$$
P\left(\mu_{+}, \mu_{-}, \mu_{3}, t\right)=\operatorname{Tr} \rho_{S}(t) \delta\left(\mu_{+}-\sigma_{+}\right) \delta\left(\mu_{-}-\sigma_{-}\right) \delta\left(\mu_{3}-\sigma_{3}\right)
$$

If the heat reservoir is only coupled with $\sigma_{3}: H_{S B}=B_{3} \sigma_{3}$, then ( 144 ) is written

$$
\frac{\partial}{\partial t} P(\mu, t)=\operatorname{Tr} \rho_{S} L^{\prime}\left(\frac{\partial}{\partial \mu}, \sigma\right) \delta(\mu)
$$

here 


$$
\begin{gathered}
\mu=\left(\mu_{+}, \mu_{-}, \mu_{3}\right), \quad \sigma=\left(\sigma_{+}, \sigma_{-}, \sigma_{3}\right) \\
L^{\prime}\left(\frac{\partial}{\partial \mu}, \sigma\right)=i L_{S} e^{-\sigma \frac{\partial}{\partial \mu}}-w_{33}^{+}\left[e^{-\sigma \frac{\partial}{\partial \mu}}, \sigma_{3}\right] \sigma_{3}+w_{33}^{-} \sigma_{3}\left[e^{-\sigma \frac{\partial}{\partial \mu}}, \sigma_{3}\right] \\
w_{33}^{+}=\pi \operatorname{Tr}_{B} B_{3} \delta\left(L_{B}\right) B_{3} \rho_{30}=-w_{33}^{-}
\end{gathered}
$$

Let's look at the free term first :

$$
\begin{aligned}
i L_{S} e^{-\sigma \frac{\partial}{\partial \mu}} & =\frac{\varepsilon}{2} i\left[\sigma_{3}, e^{-\sigma \frac{\partial}{\partial \mu}}\right] \\
& =\frac{\varepsilon}{2} i\left[\sigma_{3}, e^{-\sigma_{+} \frac{\partial}{\partial \mu_{+}}}\right] e^{-\sigma_{-} \frac{\partial}{\partial \mu_{-}}} e^{-\sigma_{3} \frac{\partial}{\partial \mu_{3}}}+\frac{\varepsilon}{2} i e^{-\sigma_{+} \frac{\partial}{\partial \mu_{+}}}\left[\sigma_{3}, e^{-\sigma_{-} \frac{\partial}{\partial \mu_{--}}}\right] e^{-\sigma_{3} \frac{\partial}{\partial \mu_{3}}}
\end{aligned}
$$

from $\left[\sigma_{3}, \sigma_{+}\right]=2 \sigma_{+}$and $\left[\sigma_{3}, \sigma_{-}\right]=-2 \sigma_{-}$, we obtain

$$
e^{\mp \alpha \sigma}+\sigma_{3} e^{ \pm \alpha \sigma}=\sigma_{3} \pm 2 \sigma_{+} \alpha, \quad e^{ \pm \alpha \sigma}-\sigma_{3} e^{ \pm \alpha \sigma}=\sigma_{3} \mp 2 \sigma_{-} \alpha
$$

therefore

$$
i L_{S} e^{-\sigma \frac{\partial}{\partial \mu}}=-\varepsilon i \sigma_{+} \frac{\partial}{\partial \mu_{+}} e^{-\sigma \frac{\partial}{\partial \mu}}+\varepsilon i e^{-\sigma_{=} \frac{\partial}{\partial \mu_{+}}} \sigma_{-} \frac{\partial}{\partial \mu_{-}} e^{-\sigma_{-} \frac{\partial}{\partial \mu_{-}}} e^{-\sigma_{3} \frac{\partial}{\partial \mu_{3}}}
$$

or

$$
\begin{aligned}
i L_{S} e^{-\sigma \frac{\partial}{\partial \mu}} \delta(\mu) & =\mathrm{i} \varepsilon \int d \beta\left(\beta_{-} \frac{\partial}{\partial \mu_{-}}-\beta_{+} \frac{\partial}{\partial \mu_{+}}\right) \delta_{N}(\beta-\sigma) \delta(\mu-\beta) \\
& =i \varepsilon\left(\frac{\partial}{\partial \mu_{-}} \mu_{-}-\frac{\partial}{\partial \mu_{+}} \mu_{+}\right) \delta_{N}(\mu-\sigma)
\end{aligned}
$$

Look at the relaxation term again. As $w_{33}^{-}=-w_{33}^{+}$, so

$$
-w_{33}^{+}\left[e^{-\sigma \frac{\partial}{\partial \mu}}, \sigma_{3}\right] \sigma_{3}+w_{33}^{-} \sigma_{3}\left[e^{-\sigma \frac{\partial}{\partial \mu}}, \sigma_{3}\right]=-w_{33}^{+}\left[e^{-\sigma \frac{\partial}{\partial \mu}}, \sigma_{3} \sigma_{3}\right]=0
$$

Thus, we obtain the Fokker-Planck equation without diffusion term :

$$
\frac{\partial}{\partial t} P(\mu, t)=i \varepsilon\left(\frac{\partial}{\partial \mu_{-}} \mu_{-}-\frac{\partial}{\partial \mu_{+}} \mu_{+}\right) P(\mu, t)
$$

Further, we can use the above ordering method to transform the motion equation of mechanical quantities in Heisenberg image into the equation of $C$-number, mechanical quantities can be written as

$$
Q=\int Q_{N}(\alpha) \delta_{N}(\alpha-a) d \alpha
$$

Thus, the time derivative of the mean value of mechanical quantity $\mathrm{Q}$ can be written as 


$$
\begin{aligned}
\frac{d}{d t}<Q(t)> & =\operatorname{Tr} \rho_{S}(0) \frac{d}{d t} Q(t) \\
& =\operatorname{Tr}_{S}(0) \int d \alpha Q_{N}\left(\alpha_{t}\right)\left\{i L_{S} \delta_{N}\left(\alpha_{t}-a_{t}\right)\right. \\
& \left.-\sum_{j, k} w_{j k}^{+}\left[\delta_{N}\left(\alpha_{t}-a_{t}\right), Q_{j}\right] Q_{k}-\sum_{j, k} w_{k j}^{-} Q_{k}\left[\delta_{N}\left(\alpha_{t}-a_{t}\right), Q_{j}\right]\right\} \\
& =\operatorname{Tr} \rho_{S}(0) \int d \alpha Q_{N}\left(\alpha_{t}\right) L^{\prime}\left(\frac{\partial}{\partial \alpha_{t}}, \alpha_{t}\right) \delta_{N}\left(\alpha_{t}-a_{t}\right)
\end{aligned}
$$

here both $\alpha_{t}$ and $a_{t}$ change with time. If the highest derivative in $L^{\prime}$ is the second order :

$$
L^{\prime}=-\sum_{j} \frac{\partial}{\partial \alpha_{j}} A_{j}(\alpha)+\sum_{j, k} \frac{\partial^{2}}{\partial \alpha_{j} \partial \alpha_{k}} D_{j k}(\alpha)
$$

then after the integral is divided into parts, we obtain

$$
\begin{aligned}
\frac{d}{d t}<Q(t)> & =\operatorname{Tr} \rho_{S}(0) \int d \alpha \delta_{N}(\alpha-a) \\
& \times\left\{\sum_{j} A_{j}(\alpha) \frac{\partial}{\partial \alpha_{j}}+\sum_{j, k} D_{j k}(\alpha) \frac{\partial^{2}}{\partial \alpha_{j} \partial \alpha_{k}}\right\} Q_{N}(\alpha, t) \\
& =\operatorname{Tr} \rho_{S}(0) \int d \alpha \delta_{N}(\alpha-a) \frac{\partial}{\partial t} Q_{N}(\alpha, t)
\end{aligned}
$$

so there should be

$$
\frac{\partial}{\partial t} Q_{N}(\alpha, t)=-\sum_{j} A_{j}(\alpha) \frac{\partial}{\partial \alpha_{j}} Q_{N}+\sum_{j, k} D_{j k}(\alpha) \frac{\partial^{2}}{\partial \alpha_{j} \partial \alpha_{k}} Q_{N}
$$

That's what we want. taking $Q=a_{k}, Q_{N}=\alpha_{t}(t)$, then formula ( 150 ) gives

$$
\frac{d}{d t} \alpha_{k}(t)=A_{k}(\alpha)
$$

Similarly, let $Q=a_{j} a_{k}(k \geq j), Q_{N}=\alpha_{j}(t) \alpha_{k}(t)$, then there should be

$$
\frac{d}{d t} \alpha_{j}(t) \alpha_{k}(t)=\alpha_{j}(t) A_{k}(\alpha)+\alpha_{k}(t) A_{j}(\alpha)+2 D_{j k}(\alpha)
$$

Because the physical image is more intuitive, formula ( 150 ) can be used as a starting point for analyzing how to adopt appropriate approximation, and then converted into the equation of operator distribution function and solved concretely.

\section{Tachyon of Upheaval in Super-Inflating Universe}

The inflation-with-tachyon model was first established in references [18]-[19]. After that, many researchers analyzed and discussed the model in detail, such as references [20]-[24], [91], [107]-[109]. The tachyon model considered below is a model called the model of inflation with assisted tachyon, see reference [90]. This model solves some difficult problems in the tachyon model of single scalar field, for example, how to realize the inflation of the universe within the effective parameter range of 
quantum field theory. The origin of the problem is shown in reference [4]. In addition, it is pointed out in reference [110] that these problems can also be solved by introducing non-minimum coupling.

The following Laplacian can be used to describe $n$ Tachyon fields that do not interact with each other:

$$
p\left(\varphi_{i}, X_{i}\right)=-\sum_{i=1}^{n} V\left(\varphi_{i}\right) \sqrt{1-2 l_{s}^{2} X_{i}},
$$

When the parameter $n$ is large enough, for example, $n \geq 10^{7}{ }^{[29]}$, the four-dimensional quantum field theory is effective, and the Friedmann equation can then be written as:

$$
H^{2}=\sum_{i=1}^{n} \frac{V\left(\varphi_{i}\right)}{3 \sqrt{1-2 l_{s}^{2} X_{i}}}=\sum_{i=1}^{n} \frac{V\left(\varphi_{i}\right)}{3 \sqrt{1-l_{s}^{2} \dot{\varphi}_{i}^{2}}} \approx \frac{n}{3} V\left(\varphi_{i}\right) .
$$

The motion equation of the inflationary field is given by the following formula:

$$
\ddot{\varphi}_{i}+3 H \dot{\varphi}_{i}\left(1-l_{s}^{2} \dot{\varphi}_{i}^{2}\right)+\frac{V_{\varphi_{i}}}{V l_{s}^{2}}\left(1-l_{s}^{2} \dot{\varphi}_{i}^{2}\right)=0 .
$$

In addition, we can get:

$$
\dot{H}=-\sum_{i=1}^{n} \frac{l_{s}^{2} \dot{\varphi}_{i}^{2} V\left(\varphi_{i}\right)}{2 \sqrt{1-l_{s}^{2} \dot{\varphi}_{i}^{2}}}, \quad c_{s}^{2}=1-l_{s}^{2} \dot{\varphi}_{i}^{2} \approx 1-\frac{2 \varepsilon_{1}}{3 n},
$$

Then, the slow roll parameters can be calculated:

$$
\begin{gathered}
\varepsilon_{1}=\frac{3}{2} \sum_{i=1}^{n} l_{s}^{2} \dot{\varphi}_{i}^{2} \approx \frac{1}{2 l_{s}^{2} n V\left(\varphi_{i}\right)}\left(\frac{V_{\varphi_{i}}}{V}\right)^{2} \approx \frac{1}{2 l_{s}^{2} n V\left(\varphi_{i}\right)}\left(\frac{V_{\varphi_{i}}}{V}\right)^{2}, \\
\varepsilon_{2} \approx-\frac{2}{l_{s}^{2} n V\left(\varphi_{i}\right)} \frac{V_{\varphi_{i} \varphi_{i}}}{V}+6 \varepsilon_{1}+O\left(\varepsilon^{2}\right), \\
s=\frac{\dot{c}_{s}}{c_{s} H}=\frac{\dot{c}_{s}^{2}}{2 c_{s}^{2} H} \approx-\frac{\dot{\varepsilon}_{1}}{3 n c_{s}^{2} H} \approx-\frac{\varepsilon_{2} \varepsilon_{1}}{3 n}\left(1-\frac{1}{3 n} \varepsilon_{1}\right)^{-1} \sim O\left(\varepsilon^{2}\right) .
\end{gathered}
$$

Considering a power-law potential energy function $V\left(\varphi_{i}\right)=\alpha \varphi_{i}^{m}$, we can get:

$$
\varepsilon_{1} \approx \frac{m^{2}}{2 l_{s}^{2} n V\left(\varphi_{i}\right) \varphi_{i}^{2}} \approx \frac{m}{2(m+2) N}, \quad \varepsilon_{2} \approx \frac{m(m+2)}{l_{s}^{2} n V\left(\varphi_{i}\right) \varphi_{i}^{2}} \approx \frac{1}{N}, \quad c_{s} \approx 1-\frac{\varepsilon_{1}}{3 n},
$$

where $N$ is the e-folding number, which is defined as follows:

$$
\begin{aligned}
N \equiv \int_{t}^{t_{\text {end }}} H d t & \approx-\int_{\varphi_{i}}^{\varphi_{i}^{e n d}} 3 H^{2} l_{s}^{2} \frac{V}{V_{\varphi_{i}}} d \varphi_{i} \\
& \approx-\frac{n l_{s}^{2} \alpha}{m} \int_{\varphi_{i}}^{\varphi_{i}^{e n d}} \varphi_{i}^{m+1} d \varphi_{i} \approx \frac{l_{s}^{2} n \alpha}{m(m+2)} \varphi_{i}^{m+2} \approx \frac{l_{s}^{2} n V\left(\varphi_{i}\right) \varphi_{i}^{2}}{m(m+2)} .
\end{aligned}
$$


Finally, the spectral indices of scalar and tensor perturbations are obtained

$$
n_{s}-1=-\frac{2 m+2}{(m+2) N}+\frac{4}{3} \lambda, \quad n_{t}=-\frac{m}{(m+2) N}+\frac{4}{3} \lambda
$$

and the tensor-scale ratio

$$
r=\frac{8 m}{(m+2) N}\left[1-\frac{m}{6 n(m+2) N}\right] \approx-\frac{8 m}{(m+2) N} .
$$

It is worth noting that when $n \geq 10^{7}$ and the parameter $\mathrm{m}$ is a positive number, the sound velocity $c_{s}$ will be very close to 1 , so that the obtained disturbance spectrum will not have a large non-Gaussianity.

According to the model of the universe with rip-rebound-inflation established in this series, photons are generally in a very strong excitation state in the cosmic inflation with the big rip-rebound cluster. This extraordinary photon system can be regarded as a process far from equilibrium, chaotic and catastrophic. The nonlinear medium of the early universe can rapidly change its oscillation behavior.

For the universe in the big impetus with rip-rebound-inflation, we consider that the arche-pulsator transits from the ground state $g$ to the excited state $\omega$ (energy band), then relaxes quickly to the metastable state (long-lived excited state) $e$, further transits to the low-energy state $g^{\prime}$, and finally returns to the ground state $g$ quickly through the stimulated radiation and spontaneous radiation. Here, the energy interval between $g^{\prime}$ and $g$ is $\Delta E>\kappa_{B} T$.

The number rate equation of the ultra-syncergistic photon in the early universe

We consider a rapidly expanding region of the universe in the big impetus with rip-reboundinflation. The number of photons in this region is $N$, the difference between the occupancy numbers of two energy levels $e$ and $g^{\prime}$ is $n=N_{e}-N_{g^{\prime}}$. When the spontaneous emission is neglected, the rate equation can be written directly.

$$
\frac{d}{d t} N=-\eta_{e} N+R n N, \quad \frac{d}{d t} n=-\eta_{a} n-R n N+E
$$

here $\eta_{e}{ }^{-1}$ is the lifetime of photons in a certain region, $\eta_{a}$ is the rate at which quasi-spherical pulsators relax from energy state $e$ to other energy states in a non-radiative way, where there are $\eta_{a} \gg>\eta_{e}, R$ is the radiation coefficient of super excitation, $E$ is the intensity of super excitation source. The steady state solution of ( 164 ) is

$$
\begin{aligned}
& N_{0}= \begin{cases}\left(E-E_{s}\right) / \eta_{e}, & E>E_{s}=\eta_{e} \eta_{a} / R \\
0, & E \leq E_{s}\end{cases} \\
& n_{0}= \begin{cases}\eta_{e} / R, & E>E_{s} \\
E / \eta_{a}, & E \leq E_{s}\end{cases}
\end{aligned}
$$

$E_{s}$ is the threshold of ultra-synergistic photons in the early universe. $E<E_{s}$, then $N_{0}=0$, that is, there is no ultra-synergistic photon. When $E>E_{s}$, near the steady state, small perturbations $\delta N=N-N_{0}$ and $\delta n=n-n_{0}$ satisfy the following system of linear equations: 


$$
\frac{d}{d t} \delta N=\frac{R}{\eta_{e}} \Delta E \delta n, \quad \frac{d}{d t} \delta n=-\frac{R}{\eta_{e}} E \delta n-\eta_{c} \delta N
$$

here $\Delta E=E-E_{s}$. let $\delta N, \delta n \sim e^{\lambda t}$, we can obtain the eigenvalue equation

$$
\lambda^{2}+\frac{R E}{\eta_{e}} \lambda+R \Delta E=0
$$

If the result is very close to the threshold, $\Delta E / E<<\eta_{a} / \eta_{e}$, there are

$$
\lambda_{1} \approx-R E / \eta_{e} \approx-\eta_{a}, \quad \lambda_{2} \approx-\eta_{e} \Delta E / E .
$$

All perturbations decay exponentially, so the steady state is always stable. $E \rightarrow E_{s}$, then $\lambda_{2} \rightarrow 0$, that is, the fluctuation (at least one disturbance mode) attenuates more and more slowly. This is a characteristic of critical slowing down, i.e., critical slowing down.

If $\eta_{a} \gg \eta_{e}$, the adiabatic approximation is taken to the quasi-spherical pulsator, we will obtain :

$$
\delta n=-\frac{\eta_{e}^{2}}{R E} \delta N
$$

Based on the instability, bifurcation and chaotic behavior revealed by existing theories and experiments for devices with two-level atoms in a non-linear medium, a simulation experiment based on the signal nonlinear oscillation circuit was designed, the hypothesis of bifurcate-chaos quantum wave was set, and the nonlinear superposition of quantum coherent states was discussed, and the analysis and calculation on the example of frequency and wavelength of the multi-level of bifurcate-chaos waves as the main component are given. Using the distribution of Wigner-Ville (WVD) as the nonlinear time-frequency distribution, we put forth the full-view Wigner distribution between classical wave function and quantum wave function, so as to put forth the quantum-classical equation of joint-varying dynamics for the spatiotemporal complexity, and then establish the quantum-classical equation of joint-varying electrodynamics for the spatiotemporal complexity.

This study involves the non-linear non-equilibrium action and process of micro-particles, and relates to the various evolution states (multi-stage multiple generation processes) of micro-particles, such as ordered state, coherent state, quasi-Bose condensation state, dissipative structure state, chaotic state, etc. ${ }^{[111]-[113]}$. The problem to be considered in this paper is how to combine the classical phase point with the coherent state wave packet of quantum, link the energy surface where the phase point moves with the energy region occupied by the coherent state, so as to study the evolution of a composite particle in the non-linear and non-equilibrium condition from the primary particle described by quantum mechanics into the multi-particle system described by classical mechanics. The further problem to be considered is how to link the matter wave described by quantum mechanics with the matter wave packet and its fluctuation described by classical mechanics, so as to study the complex evolution behavior of a composite particle under non-equilibrium conditions and in non-linear interactions.

Considering that there is a random fluctuation source $\phi(t)$ in the ultra-synergistic light field, then 


$$
\frac{d}{d t} \delta N=-\eta_{e} \frac{\Delta E}{E} \delta N+\phi(t)
$$

Let $\delta N(t=0)=0$, then the solution of formula ( 169$)$ is

$$
\delta N(t)=\int_{0}^{t} d t^{\prime} e^{-\frac{\Delta E}{E} \eta_{e}\left(t-t^{\prime}\right)} \phi\left(t^{\prime}\right) .
$$

Because $\langle\phi(t)\rangle=0$, there should be $\langle\delta N(t)\rangle=0$.

If the correlation function of $\phi(t)$ is white spectrum : $\left\langle\phi(t) \phi\left(t^{\prime}\right)\right\rangle=C \delta\left(t-t^{\prime}\right)$, the fluctuation (quadratic moment) is

$$
<\delta N^{2}(t)>=\frac{C E}{2 \eta_{e} \Delta E}\left(1-e^{-\frac{2 \Delta E}{E} \eta_{e} t}\right) \underset{t \rightarrow \infty}{\longrightarrow} \frac{C}{2 \eta_{e}} \frac{E}{\Delta E}=\frac{1}{2 \eta_{e}^{2} N_{0}} C E .
$$

When $E$ approximates $E_{s}$ (the output of ultra-synergistic light tends to zero), then the fluctuation $\sqrt{<\delta N^{2}>}$ becomes larger and larger. This phenomenon of fluctuation divergence is critical fluctuation, which is the same as that of continuous phase transition at the phase change point. In fact, it is due to the disappearance of resilience ( the first term on the right of formula ( 169 ) approximates zero ) or the appearance of soft modes.

As a random variable, the photon number $N$ of super-synergy with its distribution function $f(N)$ in the early universe satisfies the Fokker-Planck equation :

$$
\frac{\partial}{\partial t} f(N, t)=-\frac{\partial}{\partial N}\left[K_{1}(N) f\right]+\frac{1}{2} \frac{\partial^{2}}{\partial N^{2}}\left(K_{2} f\right)
$$

$K_{1}(N)$ is the friction force in Langevin equation, $K_{2}$ is the correlation strength of fluctuation force. If we take the adiabatic approximation for $n$, we get $K_{1}(N)=-\frac{\partial}{\partial N} V(N)$, here

$$
V(N)=-E N+\frac{1}{2} \eta_{e} N^{2}+\frac{\eta_{a}}{R} E \ln \left(\eta_{a}+R N\right),
$$

$K_{2}=C$. The steady-state solution of the Fokker-Planck equation ( 171 ) is

$$
f_{0}(N)=\kappa^{-1} \exp [-2 V(N) / C]
$$

$\kappa$ is the normalization factor. When $E>E_{s}, N_{0}=\Delta E / \eta_{e}$ is the minimum of the equivalent potential $V$ $(N)$. In the vicinity of $N_{0}, N=N_{0}+\delta N$, there is

$$
f_{0}(\delta N)=\kappa^{-1} \exp \left[-\frac{2}{C} V\left(N_{0}\right)-\left.\frac{1}{C} \frac{\partial^{2} V}{\partial N^{2}}\right|_{N=N_{0}} \delta N^{2}\right],
$$

This is the Gauss distribution.

The number rate equation qualitatively describes the change behavior of the output intensity of ultra-synergistic photons, but the coherence of ultra-synergistic photons is not taken into account.

\section{On Bifurcate-Chaotic Volatility of Quantum System}


Dissipative quantum systems and their irreversible processes form new problems of complexity to existing quantum mechanics ${ }^{[114]-[116]}$. The quantum hypothesis as the basis of the existing quantum mechanics is confined to the thermal equilibrium condition in the beginning, so to make quantum mechanics into incongruity with non-equilibrium statistical theory and nonlinear dynamics. For the particle with complex evolution behavior, it is necessary to again explore some of the basic concepts of quantum mechanics. In the Langevin equation for the interaction of light and atoms, the effects of various random forces (optical noises) are included, so that only the observed quantities are used in getting statistical average to random forces, it can be combined with the experimental values ${ }^{[115][117]}$. Optical chaos is another case, here there is no need to add the influence of random force to the kinetic equation. The study of optical bifurcation and chaos is a new stage in the development of nonlinear optics.

In this series, the author proposes that nonlinear oscillation experiments based on RLC circuits (Kraus J, Schafer K, Kulander K. Phys. Rev Lett, 1992, 68: 766) should be used to simulate the complex behavior and processes of particles. In the further research on the complex quantum behavior of microscopic particles, we can introduce nonlinear oscillation circuits into particle accelerators or electron guns. The studies of nonlinear oscillations have shown that a non- autonomous 2-order circuit should become chaotic, while an autonomous 1-order circuit. can not be chaotic, because it can only be transformed into an equivalent 2-order autonomous system.

By all kinds of nonlinear oscillation circuit experimental results, we can conclude that with the increase of the bifurcate stage number $n$, the nonlinear oscillation signal is in the increase $(z(n) \lambda)$ of its wavelength by $z(n)$ times of period and the decrease $(v / z(n))$ of its frequency by $z(n)$ times of period; in the $n$-th stage of bifurcation, the nonlinear oscillation signal is formed in the superposition of $z(n)$ branch signals; if the sampling divide-frequency is $1 / z(n) \Delta$, and the divide-frequency of signals is higher than $1 /[z(n+1) \Delta] H z$, then there is the lower divide- frequency appeared in divide-frequency analyses (Kraus J, Schafer K, Kulander K. Phys. Rev Lett, 1992, 68 : 766), where $z$ $(n)$ is the numerical functions associated with the bifurcate stage number $n$.

Take the sinusoidal signal $\sin _{([n])} 2 \pi\left(v / 2^{n}\right) t$ in the $n$-th stage of bifurcation as example, there should be the following property :

$$
\sin _{([n])} 2 \pi\left(v / 2^{n}\right) t=\sum_{i=1}^{2^{n}} \sin _{[n i]} 2 \pi\left(v / 2^{n}\right) t=2^{n} \sin _{[n]} 2 \pi\left(v / 2^{n}\right) t
$$

where $z(n)=2^{n}$, subcript ([n]) expresses the $n$-th stage of bifurcation.

For the $N$-th stage of bifurcation-chaotic signals in nonlinear circuits, the periodic time-domain function $x(t)$ can be expanded into a period-doubling time-domain function $x_{\{N\}}(t)$, which is expressed as the sum of the corresponding trigonometric series. For example, here are the following expressions:

$$
x_{\{N\}}(t)=A_{0}+\sum_{n=0}^{N} \sum_{m_{n}=M_{n-1}}^{M_{n}} z(n)\left[A_{m_{n}} \cos m_{n}\left(\omega_{0} / 2^{n}\right) t+2^{n} B_{m_{n}} \sin m_{n}\left(\omega_{0} / 2^{n}\right) t\right]
$$

where $n=1,2, \cdots, N ; M_{N}=\infty$.

Based on the above theoretical explanation and simulation experimental analysis, we put forth the 
following hypotheses:

1. Any particle consisting of fundamental particles in open conditions should be viewed as a Poincare system, and any particle as an unstable Poincare system in open conditions can be regarded as a nonlinear and non equilibrium system with self organization.

2. The material particles and photons in nonlinear actions should have the $N$ states of bifurcatechaotic volatility with its divide-frequency as $v / z(n)$ and wavelength as $z(n) \lambda(z(n))(n$ is the number of the branches of the $n$-th stage of bifurcation), and the material particles and photons should evolve in the bifurcate-chaotic wave under the thermal condition of non equilibrium. In particular, when $n \rightarrow \infty$, the physical particles have tge bifurcate-chaotic volatility as photons do; when $n=0$, the particles have the de Broglie volatility as photons do.

3. The particle at the $q$-th branch of the $n$-th state should have the wave with its divide- frequency as $v / z(n)$; the particle in the $n$-th state should have $z(n)$ branch waves; the particle in the $N$ stage of bifurcation should have $\sum_{n=1}^{N} z(n)$ bifurcate-chaos waves.

4. The above bifurcate-chaos volatility of the microscopic particles should be attributed to the volatility of the quantum nodes in multilayer and multiple branchs, and then can be described with the joint-varying physical quantities of the quantum nodes in the full-view space.

The basic corollary is as follows:

In general, for any complex particle under non-equilibrium conditions and nonlinear actions, the frequency and wavelength of the $n$-th bifurcate waves should be

$$
v_{[n]}=v\left[\frac{1}{z(n) \delta^{n-1}} \eta_{1}(1+n)\right], \quad \lambda_{[n]}=\lambda\left[\frac{1}{\delta^{n-1}} z(n) \eta_{1}(1+n)\right]
$$

where $\delta$ is Feigenbaum constant; $\eta_{\mathrm{o}}$ is the proportion of un-bifurcated period $\left[t_{\mathrm{o}}, t_{1}\right]$ in a specific period $\left[t_{0}, t\right]$ of evolution, and $0 \leq \eta_{0}<1 ; \eta_{1}$ is the proportion of the 1st stage bifurcated period $\left[t_{1}, t\right.$ 2] in $\left[t_{\mathrm{o}}, t\right]$. The superimposed frequency and the superimposed wavelength of the $n$-th stage bifurcate wave shall be

$$
v_{([n])}=v\left[\frac{1}{\delta^{n-1}} \eta_{1}(1+n)\right], \quad \lambda_{([n])}=\lambda\left[\frac{1}{\delta^{n-1}} \eta_{1}(1+n)\right]
$$

The frequency and wavelength of $N$ stage bifurcate wave should be

$$
\begin{aligned}
& v_{N}=v\left[\eta_{0}+\sum_{n=1}^{N} \frac{1}{z(n) \delta^{n-1}} \eta_{1}(1+n)\right], \\
& \lambda_{N}=\lambda\left[\eta_{0}+\sum_{n=1}^{N} \frac{1}{\delta^{n-1}} z(n) \eta_{1}(1+n)\right] 。
\end{aligned}
$$

For simplicity, sometimes $v_{\{\mathrm{N}\}}$ and $\lambda_{\{\mathrm{N}\}}$ can be denoted respectively as $v_{\lambda}$ and $\lambda_{\lambda}$, where $\eta_{\lambda}$ is

$$
\eta_{\lambda}=\eta_{0}+\sum_{n=1}^{N} \frac{1}{\delta^{n-1}} \eta_{1}(1+n)
$$

In the $A$-th case, by the above basic hypotheses as well as the momentum-energy relation $p=E / c$ 
of the special relativity theory and Planck's energy relation $E=h v$, it can be determined that the $N$ stage bifurcate-chaos chaotic particle and its bifurcate-chaotic wave should satisfy the relations:

$$
E_{\{N\}}=h \cdot v_{\{N\}}=\hbar \cdot \omega\left(\eta_{0}+\sum_{n=1}^{N} \frac{1}{\delta^{n-1}} \eta_{1}\right), \quad P_{\{N\}}=h P_{0} \cdot \frac{1}{\lambda_{\{N\}}}=\hbar k_{\{N\}} .
$$

where the numerical value of wave vector $k_{\{n\}}$ and wavelength $\lambda_{\{n\}}$ in $N$ stage bifurcaton should be

$$
k_{\{N\}}=2 \pi / \lambda\left(\eta_{0}+\sum_{n=1}^{N} \frac{1}{\delta^{n-1}} \eta_{1}\right), \quad \lambda_{\{N\}}=\lambda\left(\eta_{0}+\sum_{n=1}^{N} \frac{1}{\delta^{n-1}} \eta_{1}\right) .
$$

In the $B$-th case, by the above basic hypotheses as well as the momentum-energy relation $p=E / c$ of the special relativity theory and Planck's energy relation $E=h v$, it can be determined that the $N$ stage bifurcate-chaos particle and its bifurcate-chaotic wave should satisfy the following relations:

$$
E_{\{N\}}=h \cdot v_{\{N\}}=\hbar \cdot \omega\left(\eta_{0}+\sum_{n=1}^{N} \frac{1}{\delta^{n-1}} \eta_{1}(1+n)\right), \quad P_{\{N\}}=h P_{0} \cdot \frac{1}{\lambda_{\{N\}}}=\hbar k_{\{N\}}
$$

where the numerical value of wave vector $k_{\{n\}}$ and wavelength $\lambda_{\{n\}}$ in $N$ stage bifurcaton should be

$$
k_{\{N\}}=2 \pi / \lambda\left(\eta_{0}+\sum_{n=1}^{N} \frac{1}{\delta^{n-1}} \eta_{1}(1+n)\right), \lambda_{\{N\}}=\lambda\left(\eta_{0}+\sum_{n=1}^{N} \frac{1}{\delta^{n-1}} \eta_{1}(1+n)\right) .
$$

\section{Quantum Theory of Early Photons in Upheaval}

According to the model of the universe with rip-rebound-inflation set up in this series, the photon are in a upheaval under the ultra-synergy of the inflation universe, the fluctuation of phase leads to the slow drift of average phase (fluctuation dissipation theorem), and makes the spectral line have a certain width. When the energy spectrum of photon field maintains the form of Planck distribution, the relevant temperature parameter $T_{\gamma}$ is inversely proportional to the cosmic scale factor $R(t)$, i.e.

$$
T_{\gamma}(t)=\frac{A}{R(t)}=T_{\gamma_{0}}\left[\frac{R_{0}}{R(t)}\right]
$$

Therefore, it is necessary to establish the reciprocal quantum theory to discuss the spectral line width or phase drift of photons in the early cosmic upheavals. In addition, for the changes of photon statistical properties in the early cosmic upheavals, only the reciprocal quantum theory can be set up for further discussion. Below the threshold, the photon distribution in upheaval with ultra-synergy is Gauss-type: $P_{0}(\alpha) \sim e^{-|\alpha|^{2} / \bar{n}}, \bar{n}$ is average photon number. Above the threshold, the ultra-synergistic field is basically in the coherent state, and the statistics of ultra-synergistic photons obey Poisson distribution. How to determine the number of ultra-synergistic photons near the threshold from Gauss type to Poisson typer, and how to change the distribution of rapidly changing photons in various ultra-synergistic multiphoton processes, are the issues we will further explore in the future.

Consider a composite system consisting of a quantum cosmic medium and an early photon field participating in ultra-synergy : 


$$
H_{s}=\frac{\omega_{0}}{2} \sum_{j} \sigma_{3 j}, \quad H_{r}=\omega_{0} a^{\dagger} a, \quad H_{s r}=g \sum_{j}\left(a \sigma_{+j}+a^{\dagger} \sigma-j\right)
$$

here $a$ is the extinction operator of ultra-synergistic photons. The ultra-synergistic photon resonates with the quantum cosmic medium (the energy is $\omega_{0}$ ). $\sigma_{ \pm}$and $\sigma_{3}$ are Pauli matrices. The sum of $j$ is found in all ultra-synergistic media. The whole photon system in upheaval is in contact with the quantum universe as a heat reservoir, the corresponding general Master is

$$
\frac{\partial}{\partial t} \rho_{T O L}-i\left(L_{0}+L_{s r}\right) \rho_{T O L}
$$

here $L_{0}=L_{r}+L_{s}-i \theta_{s}-i \theta_{r}, \theta_{s}$ is the relaxation operator of ultra-synergistic medium and $\theta_{r}$ is the relaxation operator of ultra-synergistic photon field. If we neglect the temperature effect $(\bar{n}=0)$ of the quantum universe as a heat reservoir, then

$$
\theta_{r} \rho_{r}=-K\left\{\left[a, \rho_{r} a^{\dagger}\right]+\left[a \rho_{r}, a^{\dagger}\right]\right\}
$$

It can be inferred that the relaxation time of ultra-synergistic medium is much shorter than that ultra-synergistic photon field: $\left\|\theta_{s}\right\|>>\theta_{r} \|$.

Corresponding to the gradual approximation in semi-classical theory, we can introduce projection operators:

$$
P \rho_{T O L}=S_{0} \operatorname{Tr}_{s} \rho_{T O L}=S_{0} \rho_{r}
$$

here $S_{0}=\prod_{j} s_{0 j}, s_{0 j}=\frac{1}{2}\left(1+d_{0} \sigma_{3 j}\right)$, The excitation intensity is marked by $d_{0}$. If $Q=1-P$, then

$$
\frac{\partial}{\partial t} \rho_{r}(t)=\left(-i L_{r}-\theta_{r}-\phi\right) \rho_{r}(t)
$$

here

$$
\phi=\int_{0}^{\infty} d \tau \operatorname{Tr}_{s} L_{s r} e^{-i Q L Q \tau} L_{s r} S_{0}=-\left.i \operatorname{Tr}_{s} L_{s r} \frac{1}{Q L Q-i \varepsilon} L_{s r} S_{0}\right|_{\varepsilon \rightarrow 0^{+}}
$$

Compared with the semi-classical theory, it can be seen that at the threshold, $\theta_{r}$ should be offset with the lowest order (proportional to $g^{2}$ term) of $\phi$, so it is necessary to expand $\phi$ for $g$ ( the coupling constant in $L_{s r}$ ) to the fourth order (the third order term is eliminated in formula ( 187 ) to $s$ tracing):

$$
\frac{1}{Q L Q} \approx \frac{1}{Q L_{0} Q}-\frac{1}{Q L_{0} Q} L_{s r} \frac{1}{Q L_{0} Q}+\frac{1}{Q L_{0} Q} L_{s r} \frac{1}{Q L_{0} Q} L_{s r} \frac{1}{Q L_{0} Q}
$$

that is

$$
\phi=-i\left\{\operatorname{Tr}_{s} L_{s r} \frac{1}{L_{0}} L_{s r} S_{0}+\operatorname{Tr}_{s} L_{s r} \frac{1}{L_{0}} L_{s r} \frac{1}{L_{0}} L_{s r} \frac{1}{L_{0}} L_{s r} S_{0}\right\}
$$

In order to solve the solution of ( 186 ) concretely, the operator distribution function is introduced : 


$$
P(\bar{\alpha}, t)=\operatorname{Tr}_{r} \rho_{r}(t) \delta\left(\bar{\alpha}^{*}-a^{\dagger}\right) \delta(\bar{\alpha}-a)
$$

In the ultra-synergy, $\bar{\alpha}^{*}=\alpha^{*} e^{i \omega_{0} t}, a^{\dagger}=b^{\dagger} e^{i \omega_{0} t}$, so formula ( 186 ) can be written as

$$
\frac{\partial}{\partial t} P(\alpha, t)=\operatorname{Tr}_{r}\left\{\left(-\theta_{r}-\phi\right) \rho_{r}(t)\right\}\left(e_{N} \delta_{N}(\alpha)\right)
$$

here $e_{N}=e^{-b^{\downarrow} \frac{\partial}{\partial \alpha^{*}}} e^{-b \frac{\partial}{\partial \alpha}}$. the contribution of therm $\theta_{r}$ in formula ( 189 ) is given

$$
\left(\frac{\partial}{\partial t} P\right)_{1}=K\left(\frac{\partial}{\partial \alpha} \alpha+\frac{\partial}{\partial \alpha^{*}} \alpha^{*}\right) P(\alpha, t)
$$

In calculating term $\phi$, since we adopt resonance interaction ( i.e. $\sigma_{+j} b, \sigma_{-j} b^{\dagger}$ ), there is $\left[H_{s}+H_{r}, H\right.$ $\left.{ }_{s r}\right]=0$, so $L_{0}^{-1}$ only needs to be taken $\left(-i \theta_{s}-i \theta_{r}\right)^{-1} \approx\left(-i \theta_{s}\right)^{-1}$. It is not difficult to get:

$$
\theta_{s}^{-1} \sigma_{ \pm}=\eta_{\perp}^{-1} \sigma_{ \pm}
$$

here $\eta_{\perp}^{-1}$ is the lateral relaxation time. The exact expression of $\theta_{s}^{-1} \sigma_{3}$ is more complex, but in most cases the results are similar to the phenomenological introduction of a longitudinal relaxation time $\eta_{0}^{-1}$, so we take $\theta_{s}^{-1} \sigma_{3} \approx \frac{1}{\eta_{0}} \sigma_{3}$. It is easy to calculate the first item ( $\left.g^{2}\right)$ on the right side of ( 188 ), which is then obtained from ( 189 ):

$$
\left(\frac{\partial}{\partial t} P\right)_{2}=-\frac{N g^{2} d_{0}}{\eta_{\perp}}\left(\frac{\partial}{\partial \alpha} \alpha+\frac{\partial}{\partial \alpha^{*}} \alpha^{*}\right) P+\frac{4 N g^{2}\left(1+d_{0}\right)}{\eta_{\perp}} \frac{\partial^{2}}{\partial \alpha \partial \alpha^{*}} P
$$

here $N$ is the number of the pulsators involved in ultra-synergy. If it is not too far from the threshold,

$$
\frac{1}{\eta_{\perp}} N g^{2} d_{0}>>\frac{1}{\eta_{0} \eta_{\perp}^{2}} N g^{4} d_{0} n_{p}
$$

If $n_{p}$ is the average number of photons, only the first order derivatives of $\alpha$ ( because $\left\|\theta_{r}\right\| \sim\left\|\phi_{2}\right\|$ ) are retained. Therefore, in the expressions of $\phi_{4}$ written above, all the terms of quadratic commutation relations can be omitted (they are only the second order derivatives caused by modification of $\phi_{2}$ ). We can then write out:

$$
\left(\frac{\partial}{\partial t} P\right)_{4}=\frac{1}{\eta_{0} \eta_{\perp}^{2}} 4 N g^{4} d_{0}\left(\frac{\partial}{\partial \alpha} \alpha+\frac{\partial}{\partial \alpha^{*}} \alpha^{*}\right) \alpha \alpha^{*} P
$$

By combining ( 190 ) with ( 191 ) and ( 192 ), we obtain the following Fokker-Planck equations:

$$
\frac{\partial}{\partial t} P\left(\alpha, \alpha^{*}, t\right)=Q P\left(\alpha, \alpha^{*}, t\right)
$$

here 


$$
\begin{gathered}
Q=\left(\frac{\partial}{\partial \alpha} \alpha+\frac{\partial}{\partial \alpha^{*}} \alpha^{*}\right)\left(u+v \alpha \alpha^{*}\right)+e q \frac{\partial^{2}}{\partial \alpha \partial \alpha^{*}} \\
u=K-N g^{2} d_{0} / \eta_{\perp}, \quad v=4 N g^{4} d_{2} / \eta_{\perp} \eta_{\perp}, \quad q=N g^{2}\left(1+d_{0}\right) / \eta_{\perp}
\end{gathered}
$$

The first term in $Q$ is the drift term, and the drift coefficient is $u+v \alpha \alpha^{*}$, the second is the diffusion of distribution function caused by fluctuation (spontaneous emission is proportional to $N\left(1+d_{0}\right)$ ), which is caused by quantum effect.

The steady state solution of equation ( 193 ) is

$$
P_{0}\left(|\alpha|^{2}\right)=R^{-1} \exp \left(-\frac{u}{2 q}|\alpha|^{2}-\frac{v}{4 q}|\alpha|^{4}\right)
$$

$u=0$ is the threshold condition. Under the threshold $u>0, P\left(|\alpha|^{2}\right)$ is mainly gaussian, with $|\alpha|^{2}=0$ as the maximum. The extreme value of $P\left(|\alpha|^{2}\right)$ deviates from zero above the threshold value $u<0$, which corresponds to the appearance of ultra-synergistic light.

From the $P_{0}\left(|\alpha|^{2}\right)$ distribution curves with different $u$ values, we can clearly see the transition process from spontaneous radiation to ultra-synergistic light. Starting from $P_{0}\left(|\alpha|^{2}\right)$, the ultrasynergistic photon number distribution can also be calculated :

$$
f(n)=\int_{0}^{\infty} \frac{1}{n !}\left(\tau|\alpha|^{2}\right)^{n} e^{-\tau|\alpha|^{2}} P_{0}\left(|\alpha|^{2}\right) d|\alpha|^{2},
$$

here $\tau=T \sqrt{v / K}, T$ is the measurement time intervals. It is not difficult to see that below the threshold, $f(n)$ approximates Gauss type, while above the threshold, it approximates Poisson type.

If we want to discuss the spectrum of ultra-synergistic light, we must calculate the solution of formula ( 193 ) which changes with time, that is, its Green function. This is limited to cases far above the threshold: $|\alpha|^{2}>>|u|$. Let

$$
t^{\prime}=\sqrt{v q} t, \quad u^{\prime}=-u / \sqrt{v q}, \quad|\beta|^{2}=\sqrt{v / q}|\alpha|^{2},
$$

the formula ( 193 ) is changed to

$$
\frac{\partial P}{\partial t^{\prime}}=-\left(\frac{\partial}{\partial \beta} \beta+\frac{\partial}{\partial \beta^{*}} \beta^{*}\right)\left(u^{\prime}-|\beta|^{2}\right) P+4 q \frac{\partial^{2}}{\partial \beta \partial \beta^{*}} P
$$

Introduing the polar coordinates: $\beta=r e^{i \varphi}, d^{2} \beta=r d r d \varphi$, then the formula ( 196 ) is changed to

$$
\frac{\partial P}{\partial t^{\prime}}=\frac{1}{r} \frac{\partial}{\partial r}\left(r \frac{\partial}{\partial r} P\right)+\frac{1}{r^{2}} \frac{\partial^{2}}{\partial \varphi^{2}} P-\frac{1}{r} \frac{\partial}{\partial r}\left[\left(u^{\prime}-r^{2}\right)^{2} r^{2} P\right]
$$

If the stationary solution corresponding to $u^{\prime}$ is $P_{0}(r)$, then the stationary quadratic moment is:

$$
<\beta^{*} \beta>=\int_{0}^{\infty} r^{2} P_{0}(r) r d r \approx u^{\prime}
$$

When it is far from the threshold, the fluctuation is relatively small and can be expanded near $\sqrt{u^{\prime}}$ : 


$$
r=\sqrt{u^{\prime}}+r_{1}(t), \quad r d r \approx \sqrt{u^{\prime}} d r_{1}, \quad\left|r_{1}\right|<<\sqrt{u^{\prime}} .
$$

After omitting the term $r_{1}^{2}$ or more, there are

$$
\frac{\partial P}{\partial t^{\prime}} \approx \frac{\partial^{2}}{\partial r_{1}^{2}} P+2 u^{\prime} \frac{\partial}{\partial r_{1}}\left(r_{1}, P\right)+\frac{1}{u^{\prime}} \frac{\partial^{2}}{\partial \varphi^{2}} P
$$

The steady-state solution satisfying $P(\varphi+2 \pi)=P(\varphi)$ is $P_{s}\left(r_{1}\right)=\sqrt{\frac{u^{\prime}}{\pi}} e^{-u^{\prime} r_{1}^{2}}$, i.e. the fluctuation of amplitude is Gauss type. The Green function ( 198 ) can be further calculated:

$$
G\left(r_{1}, t^{\prime} \mid r_{10}, 0\right) \underset{t^{\prime} \rightarrow 0}{\longrightarrow} \delta\left(r_{1}-r_{10}\right) \delta\left(\varphi-\varphi_{0}\right)=\lim _{\varepsilon \rightarrow 0} \sqrt{\frac{\varepsilon}{\pi}} e^{-\varepsilon\left(r_{1}-r_{10}\right)^{2}} \sum_{n=-\infty}^{\infty} \frac{1}{2 \pi} e^{i n\left(\varphi-\varphi_{0}\right)}
$$

and then we get

$$
G=\frac{1}{2 \pi} \sqrt{\frac{\eta(t)}{\pi u^{\prime}}} \exp \left\{-\frac{\left[r_{1}-\xi(t)\right]^{2}}{\delta(t)}\right\} \sum_{n=-\infty}^{\infty} e^{i n\left(\varphi-\varphi_{0}\right)} e^{-\frac{n^{2}}{u^{\prime}}}
$$

here $\eta(t)=\delta(t)^{-1}, \delta(t)=\frac{1}{u^{\prime}}\left(1-e^{-4 u^{\prime} t}\right), \quad \xi(t)=r_{10} e^{-2 u^{\prime} t}$.

Thus, the correlation function can be calculated directly:

$$
<a^{\dagger}(t) a(0)>=e^{i \omega_{0} t-\frac{t}{u^{\prime}}}\left(u^{\prime}+\frac{1}{2 u^{\prime}} e^{-2 u^{\prime} t}\right)
$$

and the fluctuation spectrum is obtained :

$$
F(\omega)=\frac{2}{\left(\omega_{0}-\omega\right)^{2}+v q / u^{2}}+\frac{2+v q / u^{2}}{\left(\omega_{0}-\omega\right)+(v q / u+2 u)^{2} / v q}
$$

This is the superposition of two Lorentz curves, here the first one is the real ultra-synergistic optical part, its half width $\left(v q / u^{2}\right)$ is very small, which is formed by the phase drift caused by spontaneous radiation; the second one has a large half width $\left(\sim 4 u^{2} / v q\right)$, which can be regarded as the background noise field.

\section{On Particle of High-Energy Cosmic Ray from $\gamma$-Ray Burst}

One study [118] used Fermi-LAT data for three years to search for possible signals of $\gamma$-ray produced by dark matter annihilation of globular clusters NGC6388 and M 15. The Fermi group has observed the $\gamma$-ray radiation of NGC6388, and the observed $\gamma$-ray spectrum is consistent with that of millisecond pulsar. At the same time, the energy spectrum obtained by annihilating dark matter to $b b$ and then generating $\gamma$-ray is in good agreement with the observed energy spectrum. No significant $\gamma$-ray radiation was observed in globular cluster M 15. Now, the related research gives the upper limit of the flux of $\gamma$-ray produced by dark matter annihilation of NGC6388 and M 15, wherein the products of dark matter annihilation are $b b, W+W_{-}, \mu+\mu_{-}, \tau+\tau_{-}$, and monochromatic light. The upper limit of these 
fluxes limits the mass and scattering cross section of dark matter several times higher than that of Fermi group through the observation of dwarf galaxy $\gamma$-ray.

By the relevant data provided by the reports from ground-based experiments such as Fly's Eye ( Bird D J, et al. phys. Rev. lett. 1993. 712 1: 340 13404; Bird D J, et al. Astrop hys. J 1994, 424 1: 49 1502.), Haverah Park ( Lawrence M A, Reid R J O, Wat son A A. J. Phys. G. Nucl. Part. Phys 199 1, 17 5: 733757.), A GA SA ( Hayashida N, et al. Phys. Rev. Lett. 1994. 73 26: 349 13494; Takeda M, et al. Phys. Rev. Lett. 1998, 81 6: 11631166.), Yakut sk ( Efimov N N, et al. in: Proc. International Symposium on Astrop physical Aspects of $t$ he Most Energetic Cosmic Rays, eds. M. Nagano and F. Takahara, Singapore: World Scientific, 199 1, 2024; Afnasiev B N. in: Proc. of International Symposium on Extremely High Energy Cosmic Rays: Astrop physics and Future Observatories, ed. M. Nagano, Tokyo: Institute for Cosmic Ray Research, 1996, 3236.), Volcano Ranch (Linsley J. Phys. Rev. Lett. 1963, 10 4: 146148; Linsley J. in: Proc, 8t h International Cosmic Ray Conference 1963, 4: 295297.) and SU GA R ( Winn M M, et al. J. Phys. G1986 12 12: 653674.), for the need of this research on examples, this series of papers provide the multi group of data in favor of the example calculation within a reasonable range of values, as shown in Table 5 and Table 6.

It is not difficult to give an example of the initial frequency and wavelength of the major components of ultra high energy, very high energy and low energy cosmic rays. For example, on the $S^{0}$ particle with its energy $\geq 2.3 \times 10^{20} \mathrm{eV}$, it gives the calculating results of the initial frequency and wavelength:

$$
\nu=\frac{E}{h}=\frac{3.489 \times 10^{20} \mathrm{eV}}{6.626 \times 10^{-34} \mathrm{~J} \cdot S}, \lambda=\frac{h}{p}=\frac{h c}{E}=\frac{6.626 \times 10^{-34} \mathrm{~J} \cdot \mathrm{S} \times c}{3.489 \times 10^{20} \mathrm{eV}} .
$$

It is not difficult to give an example of the frequency and wavelength of the un-bifurcated and the $n$-th stage bifurcate wave of the $S^{0}$ particle in ultra high energy cosmic rays. For example, on the $S^{0}$ particle in ultra high energy cosmic rays, it gives the calculating results of the frequency and wavelength of the 2nd stage bifurcated wave when the number of bifurcation is 2 :

$$
\begin{aligned}
& v_{[2]}=3 \times 0.45 \times \frac{1}{4 \times 4.6692} \times\left(\frac{3.489 \times 10^{20} \mathrm{eV}}{6.626 \times 10^{-34} \mathrm{~J} \cdot \mathrm{S}}\right), \\
& \lambda_{[2]}=3 \times 4 \times 0.45 \times \frac{1}{4.6692} \times\left(\frac{6.626 \times 10^{-34} \mathrm{~J} \cdot \mathrm{S} \times \mathrm{c}}{3.489 \times 10^{20} \mathrm{eV}}\right) .
\end{aligned}
$$

By expressions ( 177 ), ( 178 ) and ( 179 ), Table 5 and Table 6 give an example of the frequency and wavelength of the $N$ stage bifurcate-chaotic wave of the major components of cosmic rays.

By the relevant data provided by the reports from ground-based experiments such as Fly's Eye ( Bird D J, et al. phys. Rev. lett. 1993; Astrop hys. J 1994), Haverah Park (Lawrence M A, et al. Phys. G. 1991), A GA SA ( Hayashida N, et al. Phys. Rev. Lett. 1994; Takeda M, et al. Phys. Rev. Lett. 1998), Yakut sk ( Efimov N N, et al.; Afnasiev B N., 1996), Volcano Ranch ( Linsley J. Phys. Rev. Lett. 1963; Linsley J., 1963) and SU GA R ( Winn M M, et al. J. Phys. G, 1986), for the need of this research on examples, this series of papers provide the multi group of data in favor of the calculation of examples within a reasonable range of values, as shown in Table 7 and Table 8.

It is not difficult to give an example of the initial energy and momentum of the major components of ultra high energy, very high energy and low energy cosmic rays. For example, on the $S^{0}$ particle with its energy $\geq 2.3 \times 10^{20} \mathrm{eV}$, it gives the calculating results of the initial energy and momentum : 


$$
E=\frac{v}{h}=\frac{3.489 \times 10^{20} e V}{6.626 \times 10^{-34} \mathrm{~J} \cdot S} / h, \quad p=\frac{h}{\lambda}=h / \frac{6.626 \times 10^{-34} \mathrm{~J} \cdot S \times c}{3.489 \times 10^{20} \mathrm{eV}} .
$$

It is not difficult to give an example of the energy and momentum of the unbifurcated and $n$-th bifurcate wave of the $S^{0}$ particle in ultra high energy cosmic rays. For example, on the $S^{0}$ particle in ultra high energy cosmic rays, it gives the calculating results of the energy and momentum of the 2 nd stage

Table 5 Example : Frequency and Wavelength of the $N$ Stage Bifurcate-Chaotic Wave of the Major Components of Ultra-High Energy Cosmic Rays

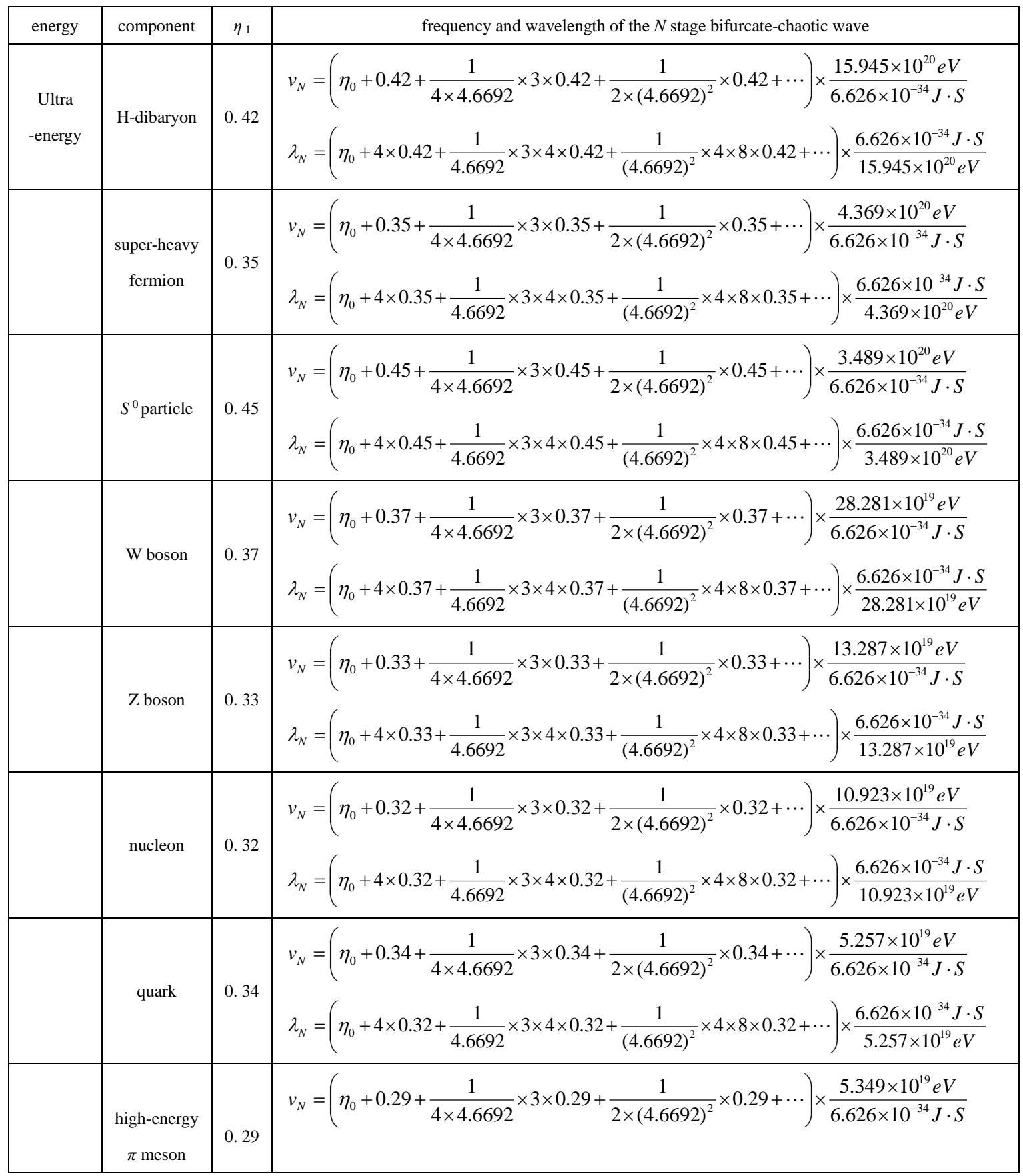




\begin{tabular}{|c|c|c|c|c|c|}
\hline & & $\lambda_{N}=$ & $\left(\eta_{0}+4 \times 0.29+\frac{1}{4.6692} \times 3 \times 4 \times 0.29+\right.$ & $\frac{1}{(4.6692)^{2}} \times 4 \times 8 \times 0.29+\cdot$ & $\times \frac{6.626 \times 10^{-34} \mathrm{~J} \cdot S}{5.349 \times 10^{19} \mathrm{eV}}$ \\
\hline $\begin{array}{l}\text { high-energy } \\
\gamma \text { meson }\end{array}$ & 0.28 & $\begin{array}{l}v_{N}= \\
\lambda_{N}=\end{array}$ & $\left\{\begin{array}{l}\eta_{0}+0.28+\frac{1}{4 \times 4.6692} \times 3 \times 0.28+ \\
\eta_{0}+4 \times 0.28+\frac{1}{4.6692} \times 3 \times 4 \times 0.28+\end{array}\right.$ & $\begin{array}{l}\left.\frac{1}{2 \times(4.6692)^{2}} \times 0.28+\cdots\right) \times \\
\frac{1}{(4.6692)^{2}} \times 4 \times 8 \times 0.28+\cdot \cdot\end{array}$ & $\begin{array}{l}\frac{6.355 \times 10^{18} \mathrm{eV}}{6.626 \times 10^{-34} \mathrm{~J} \cdot \mathrm{S}} \\
\cdot \frac{6.626 \times 10^{-34} \mathrm{~J} \cdot \mathrm{S}}{6.355 \times 10^{18} \mathrm{eV}}\end{array}$ \\
\hline
\end{tabular}

Table 6 Example : Frequency and Wavelength of the $N$ Stage Bifurcate-Chaotic Wave of the Major Components of Very High Energy and Low Energy Cosmic Rays

\begin{tabular}{|c|c|c|c|}
\hline energy & component & $\eta_{1}$ & frequency and wavelength of the $N$ stage bifurcate-chaotic wave \\
\hline $\begin{array}{l}\text { very } \\
\text { energy }\end{array}$ & proton & 0.28 & $\begin{array}{l}v_{N}=\left(\eta_{0}+0.28+\frac{1}{4 \times 4.6692} \times 3 \times 0.28+\frac{1}{2 \times(4.6692)^{2}} \times 0.28+\cdots\right) \times \frac{2.473 \times 10^{19} \mathrm{eV}}{6.626 \times 10^{-34} \mathrm{~J} \cdot \mathrm{S}} \\
\lambda_{N}=\left(\eta_{0}+4 \times 0.28+\frac{1}{4.6692} \times 3 \times 4 \times 0.28+\frac{1}{(4.6692)^{2}} \times 4 \times 8 \times 0.28+\cdots\right) \times \frac{6.626 \times 10^{-34} \mathrm{~J} \cdot \mathrm{S}}{2.473 \times 10^{19} \mathrm{eV}}\end{array}$ \\
\hline & neutron & 0.30 & $\begin{array}{l}v_{N}=\left(\eta_{0}+0.30+\frac{1}{4 \times 4.6692} \times 3 \times 0.30+\frac{1}{2 \times(4.6692)^{2}} \times 0.30+\cdots\right) \times \frac{3.488 \times 10^{19} \mathrm{eV}}{6.626 \times 10^{-34} \mathrm{~J} \cdot S} \\
\lambda_{N}=\left(\eta_{0}+4 \times 0.30+\frac{1}{4.6692} \times 3 \times 4 \times 0.30+\frac{1}{(4.6692)^{2}} \times 4 \times 8 \times 0.30+\cdots\right) \times \frac{6.626 \times 10^{-34} \mathrm{~J} \cdot S}{3.488 \times 10^{19} \mathrm{eV}}\end{array}$ \\
\hline & $\begin{array}{c}\text { Light } \\
\text { nuclei } \mathrm{H}\end{array}$ & 0.25 & $\begin{array}{l}v_{N}=\left(\eta_{0}+0.25+\frac{1}{4 \times 4.6692} \times 3 \times 0.25+\frac{1}{2 \times(4.6692)^{2}} \times 0.25+\cdots\right) \times \frac{19.029 \times 10^{15} \mathrm{eV}}{6.626 \times 10^{-34} \mathrm{~J} \cdot \mathrm{S}} \\
\lambda_{N}=\left(\eta_{0}+4 \times 0.25+\frac{1}{4.6692} \times 3 \times 4 \times 0.25+\frac{1}{(4.6692)^{2}} \times 4 \times 8 \times 0.25+\cdots\right) \times \frac{6.626 \times 10^{-34} \mathrm{~J} \cdot \mathrm{S}}{19.029 \times 10^{15} \mathrm{eV}}\end{array}$ \\
\hline & $\begin{array}{c}\text { Light } \\
\text { nuclei He }\end{array}$ & 0.23 & $\begin{array}{l}v_{N}=\left(\eta_{0}+0.23+\frac{1}{4 \times 4.6692} \times 3 \times 0.23+\frac{1}{2 \times(4.6692)^{2}} \times 0.23+\cdots\right) \times \frac{4.298 \times 10^{17} \mathrm{eV}}{6.626 \times 10^{-34} \mathrm{~J} \cdot \mathrm{S}} \\
\lambda_{N}=\left(\eta_{0}+4 \times 0.23+\frac{1}{4.6692} \times 3 \times 4 \times 0.23+\frac{1}{(4.6692)^{2}} \times 4 \times 8 \times 0.23+\cdots\right) \times \frac{6.626 \times 10^{-34} \mathrm{~J} \cdot \mathrm{S}}{4.298 \times 10^{17} \mathrm{eV}}\end{array}$ \\
\hline & $X$-ray & 0.31 & $\begin{array}{l}v_{N}=\left(\eta_{0}+0.31+\frac{1}{4 \times 4.6692} \times 3 \times 0.31+\frac{1}{2 \times(4.6692)^{2}} \times 0.31+\cdots\right) \times \frac{13.345 \times 10^{5} \mathrm{eV}}{6.626 \times 10^{-34} \mathrm{~J} \cdot \mathrm{S}} \\
\lambda_{N}=\left(\eta_{0}+4 \times 0.31+\frac{1}{4.6692} \times 3 \times 4 \times 0.31+\frac{1}{(4.6692)^{2}} \times 4 \times 8 \times 0.31+\cdots\right) \times \frac{6.626 \times 10^{-34} \mathrm{~J} \cdot \mathrm{S}}{13.345 \times 10^{5} \mathrm{eV}}\end{array}$ \\
\hline & $\pi$ meson & 0.26 & $\begin{array}{l}v_{N}=\left(\eta_{0}+0.26+\frac{1}{4 \times 4.6692} \times 3 \times 0.26+\frac{1}{2 \times(4.6692)^{2}} \times 0.26+\cdots\right) \times \frac{3.480 \times 10^{6} \mathrm{eV}}{6.626 \times 10^{-34} \mathrm{~J} \cdot \mathrm{S}} \\
\lambda_{N}=\left(\eta_{0}+4 \times 0.26+\frac{1}{4.6692} \times 3 \times 4 \times 0.26+\frac{1}{(4.6692)^{2}} \times 4 \times 8 \times 0.26+\cdots\right) \times \frac{6.626 \times 10^{-34} \mathrm{~J} \cdot S}{3.480 \times 10^{6} \mathrm{eV}}\end{array}$ \\
\hline & $\begin{array}{l}\text { positron } \\
\text {-electron }\end{array}$ & 0.25 & $\begin{array}{l}v_{N}=\left(\eta_{0}+0.25+\frac{1}{4 \times 4.6692} \times 3 \times 0.25+\frac{1}{2 \times(4.6692)^{2}} \times 0.25+\cdots\right) \times \frac{5.110 \times 10^{5} \mathrm{eV}}{6.626 \times 10^{-34} \mathrm{~J} \cdot \mathrm{S}} \\
\lambda_{N}=\left(\eta_{0}+4 \times 0.25+\frac{1}{4.6692} \times 3 \times 4 \times 0.25+\frac{1}{(4.6692)^{2}} \times 4 \times 8 \times 0.25+\cdots\right) \times \frac{6.626 \times 10^{-34} \mathrm{~J} \cdot \mathrm{S}}{5.110 \times 10^{5} \mathrm{eV}}\end{array}$ \\
\hline & low energy & & $v_{N}=\left(\eta_{0}+0.22+\frac{1}{4 \times 4.6692} \times 3 \times 0.22+\frac{1}{2 \times(4.6692)^{2}} \times 0.22+\cdots\right) \times \frac{15.320 \times \mathrm{KeV}}{6.626 \times 10^{-34} \mathrm{~J} \cdot S}$ \\
\hline
\end{tabular}




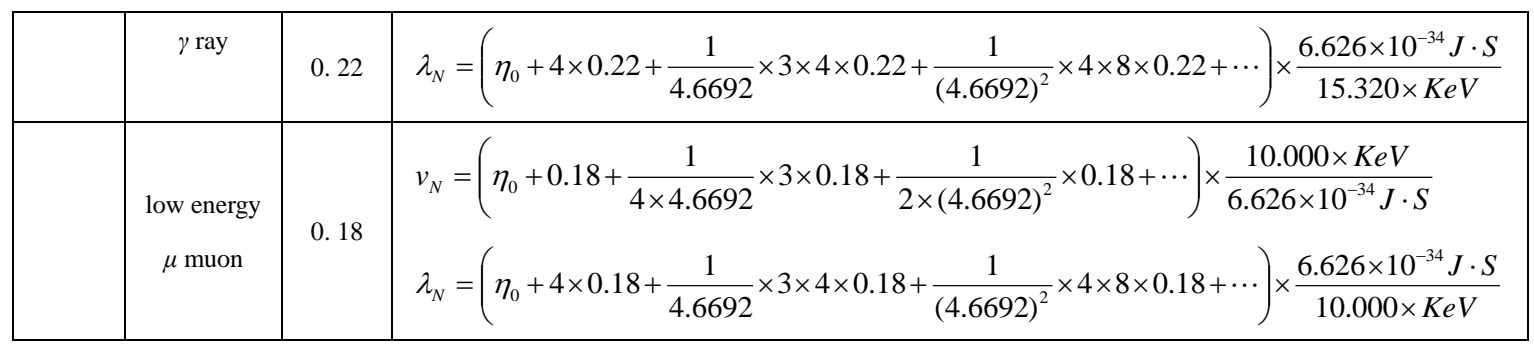

Table 7 Example : Energy and Momentum of the $N$ Stage Bifurcate-Chaotic Wave of the Major Components of Ultra-High Energy Cosmic Rays

\begin{tabular}{|c|c|c|c|}
\hline energy & component & $\eta_{1}$ & energy and momentum of the $N$ stage bifurcate-chaotic wave \\
\hline $\begin{array}{l}\text { Ultra } \\
\text {-energy }\end{array}$ & H-dibaryon & 0.42 & $\begin{array}{l}E_{N}=\left(\eta_{0}+0.42+\frac{1}{4 \times 4.6692} \times 3 \times 0.42+\frac{1}{2 \times(4.6692)^{2}} \times 0.42+\cdots\right) \times \frac{15.945 \times 10^{20} \mathrm{eV}}{6.626 \times 10^{-34} \mathrm{~J} \cdot \mathrm{S}} / \mathrm{h} \\
p_{N}=h /\left(\eta_{0}+4 \times 0.42+\frac{1}{4.6692} \times 3 \times 4 \times 0.42+\frac{1}{(4.6692)^{2}} \times 4 \times 8 \times 0.42+\cdots\right) \times \frac{6.626 \times 10^{-34} \mathrm{~J} \cdot \mathrm{S}}{15.945 \times 10^{20} \mathrm{eV}}\end{array}$ \\
\hline & $\begin{array}{l}\text { super-heavy } \\
\text { fermion }\end{array}$ & 0.35 & $\begin{array}{l}E_{N}=\left(\eta_{0}+0.35+\frac{1}{4 \times 4.6692} \times 3 \times 0.35+\frac{1}{2 \times(4.6692)^{2}} \times 0.35+\cdots\right) \times \frac{4.369 \times 10^{20} \mathrm{eV}}{6.626 \times 10^{-34} \mathrm{~J} \cdot \mathrm{S}} / \mathrm{h} \\
p_{N}=h /\left(\eta_{0}+4 \times 0.35+\frac{1}{4.6692} \times 3 \times 4 \times 0.35+\frac{1}{(4.6692)^{2}} \times 4 \times 8 \times 0.35+\cdots\right) \times \frac{6.626 \times 10^{-34} \mathrm{~J} \cdot S}{4.369 \times 10^{20} \mathrm{eV}}\end{array}$ \\
\hline & $S^{0}$ particle & 0.45 & $\begin{array}{l}E_{N}=\left(\eta_{0}+0.45+\frac{1}{4 \times 4.6692} \times 3 \times 0.45+\frac{1}{2 \times(4.6692)^{2}} \times 0.45+\cdots\right) \times \frac{3.489 \times 10^{20} \mathrm{eV}}{6.626 \times 10^{-34} \mathrm{~J} \cdot \mathrm{S}} / \mathrm{h} \\
p_{N}=h /\left(\eta_{0}+4 \times 0.45+\frac{1}{4.6692} \times 3 \times 4 \times 0.45+\frac{1}{(4.6692)^{2}} \times 4 \times 8 \times 0.45+\cdots\right) \times \frac{6.626 \times 10^{-34} \mathrm{~J} \cdot S}{3.489 \times 10^{20} \mathrm{eV}}\end{array}$ \\
\hline & W boson & 0.37 & $\begin{array}{l}E_{N}=\left(\eta_{0}+0.37+\frac{1}{4 \times 4.6692} \times 3 \times 0.37+\frac{1}{2 \times(4.6692)^{2}} \times 0.37+\cdots\right) \times \frac{28.281 \times 10^{19} \mathrm{eV}}{6.626 \times 10^{-34} \mathrm{~J} \cdot \mathrm{S}} / \mathrm{h} \\
p_{N}=h /\left(\eta_{0}+4 \times 0.37+\frac{1}{4.6692} \times 3 \times 4 \times 0.37+\frac{1}{(4.6692)^{2}} \times 4 \times 8 \times 0.37+\cdots\right) \times \frac{6.626 \times 10^{-34} \mathrm{~J} \cdot S}{28.281 \times 10^{19} \mathrm{eV}}\end{array}$ \\
\hline & $\mathrm{Z}$ boson & 0.33 & $\begin{array}{l}E_{N}=\left(\eta_{0}+0.33+\frac{1}{4 \times 4.6692} \times 3 \times 0.33+\frac{1}{2 \times(4.6692)^{2}} \times 0.33+\cdots\right) \times \frac{13.287 \times 10^{19} \mathrm{eV}}{6.626 \times 10^{-34} \mathrm{~J} \cdot \mathrm{S}} / \mathrm{h} \\
p_{N}=h /\left(\eta_{0}+4 \times 0.33+\frac{1}{4.6692} \times 3 \times 4 \times 0.33+\frac{1}{(4.6692)^{2}} \times 4 \times 8 \times 0.33+\cdots\right) \times \frac{6.626 \times 10^{-34} \mathrm{~J} \cdot \mathrm{S}}{13.287 \times 10^{19} \mathrm{eV}}\end{array}$ \\
\hline & nucleon & 0.32 & $\begin{array}{l}E_{N}=\left(\eta_{0}+0.32+\frac{1}{4 \times 4.6692} \times 3 \times 0.32+\frac{1}{2 \times(4.6692)^{2}} \times 0.32+\cdots\right) \times \frac{10.923 \times 10^{19} \mathrm{eV}}{6.626 \times 10^{-34} \mathrm{~J} \cdot \mathrm{S}} / \mathrm{h} \\
p_{N}=h /\left(\eta_{0}+4 \times 0.32+\frac{1}{4.6692} \times 3 \times 4 \times 0.32+\frac{1}{(4.6692)^{2}} \times 4 \times 8 \times 0.32+\cdots\right) \times \frac{6.626 \times 10^{-34} \mathrm{~J} \cdot \mathrm{S}}{10.923 \times 10^{19} \mathrm{eV}}\end{array}$ \\
\hline & quark & 0.34 & $\begin{array}{l}E_{N}=\left(\eta_{0}+0.34+\frac{1}{4 \times 4.6692} \times 3 \times 0.34+\frac{1}{2 \times(4.6692)^{2}} \times 0.34+\cdots\right) \times \frac{5.257 \times 10^{19} \mathrm{eV}}{6.626 \times 10^{-34} \mathrm{~J} \cdot \mathrm{S}} / \mathrm{h} \\
p_{N}=h /\left(\eta_{0}+4 \times 0.32+\frac{1}{4.6692} \times 3 \times 4 \times 0.32+\frac{1}{(4.6692)^{2}} \times 4 \times 8 \times 0.32+\cdots\right) \times \frac{6.626 \times 10^{-34} \mathrm{~J} \cdot S}{5.257 \times 10^{19} \mathrm{eV}}\end{array}$ \\
\hline & high-energy & & $E_{N}=\left(\eta_{0}+0.29+\frac{1}{4 \times 4.6692} \times 3 \times 0.29+\frac{1}{2 \times(4.6692)^{2}} \times 0.29+\cdots\right) \times \frac{5.349 \times 10^{19} \mathrm{eV}}{6.626 \times 10^{-34} \mathrm{~J} \cdot \mathrm{S}} / \mathrm{h}$ \\
\hline
\end{tabular}




\begin{tabular}{|c|c|c|c|}
\hline & $\pi$ meson & 0.29 & $p_{N}=h\left(\eta_{0}+4 \times 0.29+\frac{1}{4.6692} \times 3 \times 4 \times 0.29+\frac{1}{(4.6692)^{2}} \times 4 \times 8 \times 0.29+\cdots\right) \times \frac{6.626 \times 10^{-34} \mathrm{~J} \cdot \mathrm{S}}{5.349 \times 10^{19} \mathrm{eV}}$ \\
\hline $\begin{array}{c}\gamma \text { high-energy } \\
\gamma \text { meson }\end{array}$ & 0.28 & $E_{N}=\left(\eta_{0}+0.28+\frac{1}{4 \times 4.6692} \times 3 \times 0.28+\frac{1}{2 \times(4.6692)^{2}} \times 0.28+\cdots\right) \times \frac{6.355 \times 10^{18} \mathrm{eV}}{6.626 \times 10^{-34} \mathrm{~J} \cdot \mathrm{S}} / \mathrm{h}$ \\
$p_{N}=h /\left(\eta_{0}+4 \times 0.28+\frac{1}{4.6692} \times 3 \times 4 \times 0.28+\frac{1}{(4.6692)^{2}} \times 4 \times 8 \times 0.28+\cdots\right) \times \frac{6.626 \times 10^{-34} \mathrm{~J} \cdot \mathrm{S}}{6.355 \times 10^{18} \mathrm{eV}}$ \\
\hline
\end{tabular}

Table 8 Example : Energy and Momentum of the $N$ Stage Bifurcate-Chaotic Wave of the Major Components of Very High Energy and Low Energy Cosmic Rays

\begin{tabular}{|c|c|c|c|}
\hline energy & component & $\eta_{1}$ & energy and momentum of the $N$ stage bifurcate-chaotic wave \\
\hline $\begin{array}{l}\text { very high } \\
\text { energy }\end{array}$ & proton & 0.28 & $\begin{array}{l}E_{N}=\left(\eta_{0}+0.28+\frac{1}{4 \times 4.6692} \times 3 \times 0.28+\frac{1}{2 \times(4.6692)^{2}} \times 0.28+\cdots\right) \times \frac{2.473 \times 10^{19} \mathrm{eV}}{6.626 \times 10^{-34} \mathrm{~J} \cdot \mathrm{S}} / \mathrm{h} \\
p_{N}=h /\left(\eta_{0}+4 \times 0.28+\frac{1}{4.6692} \times 3 \times 4 \times 0.28+\frac{1}{(4.6692)^{2}} \times 4 \times 8 \times 0.28+\cdots\right) \times \frac{6.626 \times 10^{-34} \mathrm{~J} \cdot \mathrm{S}}{2.473 \times 10^{19} \mathrm{eV}}\end{array}$ \\
\hline & neutron & 0.30 & $\begin{array}{l}E_{N}=\left(\eta_{0}+0.30+\frac{1}{4 \times 4.6692} \times 3 \times 0.30+\frac{1}{2 \times(4.6692)^{2}} \times 0.30+\cdots\right) \times \frac{3.488 \times 10^{19} \mathrm{eV}}{6.626 \times 10^{-34} \mathrm{~J} \cdot \mathrm{S}} / \mathrm{h} \\
p_{N}=h /\left(\eta_{0}+4 \times 0.30+\frac{1}{4.6692} \times 3 \times 4 \times 0.30+\frac{1}{(4.6692)^{2}} \times 4 \times 8 \times 0.30+\cdots\right) \times \frac{6.626 \times 10^{-34} \mathrm{~J} \cdot \mathrm{S}}{3.488 \times 10^{19} \mathrm{eV}}\end{array}$ \\
\hline & $\begin{array}{l}\text { light nuclei } \\
\qquad \mathrm{H}\end{array}$ & 0.25 & $\begin{array}{l}E_{N}=\left(\eta_{0}+0.25+\frac{1}{4 \times 4.6692} \times 3 \times 0.25+\frac{1}{2 \times(4.6692)^{2}} \times 0.25+\cdots\right) \times \frac{19.029 \times 10^{15} \mathrm{eV}}{6.626 \times 10^{-34} \mathrm{~J} \cdot \mathrm{S}} / \mathrm{h} \\
p_{N}=h\left(\eta_{0}+4 \times 0.25+\frac{1}{4.6692} \times 3 \times 4 \times 0.25+\frac{1}{(4.6692)^{2}} \times 4 \times 8 \times 0.25+\cdots\right) \times \frac{6.626 \times 10^{-34} \mathrm{~J} \cdot \mathrm{S}}{19.029 \times 10^{15} \mathrm{eV}}\end{array}$ \\
\hline & $\begin{array}{l}\text { light nuclei } \\
\qquad \mathrm{He}\end{array}$ & 0.23 & $\begin{array}{l}E_{N}=\left(\eta_{0}+0.23+\frac{1}{4 \times 4.6692} \times 3 \times 0.23+\frac{1}{2 \times(4.6692)^{2}} \times 0.23+\cdots\right) \times \frac{4.298 \times 10^{17} \mathrm{eV}}{6.626 \times 10^{-34} \mathrm{~J} \cdot \mathrm{S}} / \mathrm{h} \\
p_{N}=h /\left(\eta_{0}+4 \times 0.23+\frac{1}{4.6692} \times 3 \times 4 \times 0.23+\frac{1}{(4.6692)^{2}} \times 4 \times 8 \times 0.23+\cdots\right) \times \frac{6.626 \times 10^{-34} \mathrm{~J} \cdot S}{4.298 \times 10^{17} \mathrm{eV}}\end{array}$ \\
\hline \multirow[t]{2}{*}{$\begin{array}{c}\text { low } \\
\text { energy }\end{array}$} & $X$-ray & 0.31 & $\begin{array}{l}E_{N}=\left(\eta_{0}+0.31+\frac{1}{4 \times 4.6692} \times 3 \times 0.31+\frac{1}{2 \times(4.6692)^{2}} \times 0.31+\cdots\right) \times \frac{13.345 \times 10^{5} \mathrm{eV}}{6.626 \times 10^{-34} \mathrm{~J} \cdot \mathrm{S}} / \mathrm{h} \\
p_{N}=h /\left(\eta_{0}+4 \times 0.31+\frac{1}{4.6692} \times 3 \times 4 \times 0.31+\frac{1}{(4.6692)^{2}} \times 4 \times 8 \times 0.31+\cdots\right) \times \frac{6.626 \times 10^{-34} \mathrm{~J} \cdot \mathrm{S}}{13.345 \times 10^{5} \mathrm{eV}}\end{array}$ \\
\hline & $\pi$ meson & 0.26 & $\begin{array}{l}E_{N}=\left(\eta_{0}+0.26+\frac{1}{4 \times 4.6692} \times 3 \times 0.26+\frac{1}{2 \times(4.6692)^{2}} \times 0.26+\cdots\right) \times \frac{3.480 \times 10^{6} \mathrm{eV}}{6.626 \times 10^{-34} \mathrm{~J} \cdot \mathrm{S}} / \mathrm{h} \\
p_{N}=h /\left(\eta_{0}+4 \times 0.26+\frac{1}{4.6692} \times 3 \times 4 \times 0.26+\frac{1}{(4.6692)^{2}} \times 4 \times 8 \times 0.26+\cdots\right) \times \frac{6.626 \times 10^{-34} \mathrm{~J} \cdot \mathrm{S}}{3.480 \times 10^{6} \mathrm{eV}}\end{array}$ \\
\hline & $\begin{array}{l}\text { positron } \\
\text {-electron }\end{array}$ & 0.25 & $\begin{array}{l}E_{N}=\left(\eta_{0}+0.25+\frac{1}{4 \times 4.6692} \times 3 \times 0.25+\frac{1}{2 \times(4.6692)^{2}} \times 0.25+\cdots\right) \times \frac{5.110 \times 10^{5} \mathrm{eV}}{6.626 \times 10^{-34} \mathrm{~J} \cdot \mathrm{S}} / \mathrm{h} \\
p_{N}=h\left(\eta_{0}+4 \times 0.25+\frac{1}{4.6692} \times 3 \times 4 \times 0.25+\frac{1}{(4.6692)^{2}} \times 4 \times 8 \times 0.25+\cdots\right) \times \frac{6.626 \times 10^{-34} \mathrm{~J} \cdot \mathrm{S}}{5.110 \times 10^{5} \mathrm{eV}}\end{array}$ \\
\hline & low energy & & $E_{N}=\left(\eta_{0}+0.22+\frac{1}{4 \times 4.6692} \times 3 \times 0.22+\frac{1}{2 \times(4.6692)^{2}} \times 0.22+\cdots\right) \times \frac{15.320 \times \mathrm{KeV}}{6.626 \times 10^{-34} \mathrm{~J} \cdot S} / \mathrm{h}$ \\
\hline
\end{tabular}




\begin{tabular}{|c|c|c|c|}
\hline & $\gamma$ ray & 0.22 & $p_{N}=h /\left(\eta_{0}+4 \times 0.22+\frac{1}{4.6692} \times 3 \times 4 \times 0.22+\frac{1}{(4.6692)^{2}} \times 4 \times 8 \times 0.22+\cdots\right) \times \frac{6.626 \times 10^{-34} \mathrm{~J} \cdot \mathrm{S}}{15.320 \times \mathrm{KeV}}$ \\
\hline $\begin{array}{c}\text { low energy } \\
\mu \text { ray }\end{array}$ & 0.18 & $E_{N}=\left(\eta_{0}+0.18+\frac{1}{4 \times 4.6692} \times 3 \times 0.18+\frac{1}{2 \times(4.6692)^{2}} \times 0.18+\cdots\right) \times \frac{10.000 \times \mathrm{KeV}}{6.626 \times 10^{-34} \mathrm{~J} \cdot \mathrm{S}} / \mathrm{h}$ \\
& $p_{N}=h /\left(\eta_{0}+4 \times 0.18+\frac{1}{4.6692} \times 3 \times 4 \times 0.18+\frac{1}{(4.6692)^{2}} \times 4 \times 8 \times 0.18+\cdots\right) \times \frac{6.626 \times 10^{-34} \mathrm{~J} \cdot \mathrm{S}}{10.000 \times \mathrm{KeV}}$ \\
\hline
\end{tabular}

$$
\begin{aligned}
& E_{[2]}=3 \times 0.45 \times \frac{1}{4 \times 4.6692} \times\left(\frac{3.489 \times 10^{20} \mathrm{eV}}{6.626 \times 10^{-34} \mathrm{~J} \cdot S}\right) / h, \\
& p_{[2]}=h / 3 \times 4 \times 0.45 \times \frac{1}{4.6692} \times\left(\frac{6.626 \times 10^{-34} \mathrm{~J} \cdot S \times c}{3.489 \times 10^{20} \mathrm{eV}}\right) .
\end{aligned}
$$

It is not difficult to give an example of the energy and momentum of the unbifurcated and $n$-th bifurcate wave of the $\mathrm{W}$ boson in ultra high energy cosmic rays. For example, on the $\mathrm{W}$ boson in ultra high energy cosmic rays, it gives the calculating results of the energy and momentum of the 3 nd stage bifurcated wave when the number of bifurcation is 3 :

$$
\begin{aligned}
& E_{[3]}=4 \times 0.37 \times \frac{1}{8 \times(4.6692)^{2}} \times\left(\frac{28.281 \times 10^{19} \mathrm{eV}}{6.626 \times 10^{-34} \mathrm{~J} \cdot S}\right) / h, \\
& p_{[3]}=h / 4 \times 8 \times 0.37 \times \frac{1}{(4.6692)^{2}} \times\left(\frac{6.626 \times 10^{-34} \mathrm{~J} \cdot \mathrm{S} \times c}{28.281 \times 10^{19} \mathrm{eV}}\right) .
\end{aligned}
$$

Table 7 and Table 8 give an example of the energy and momentum of the $N$ stage bifurcatechaotic wave of the major components of cosmic rays.

For both the macroscopic quantum system and the microscopic quantum system, the influence of environment (external field) and the interaction between quantum system and environment must be considered. In the analysis of this paper, electrons, quarks and so on are the secondary particles with a certain dividing frequency and wavelet on the bifurcate-chaotic wave of an original particle. In the viewpoint of the quantum mechanics of nonlinear non-equilibrium, these fields such as electromagnetic field and electronic field is not basic, which can only be used to approximately describe the phenomenon, and is effective only in so low energy that the high frequency vibration mode of the string can not be excited. In the simplest form of the original standard model, the mass of particle is related to some constants. The latter has determined the interaction intensity between various elementary particles and a new field all over the whole universe.

As a complex plasma system, the cosmic ray of ultra high energy interacts with its environment, just like the other macroscopic systems. In general, it is irreversible and tends to erase the coherence of quantum. Take SQUID or CBJ for example, even in $T \ll T_{c}$, the number of normal electrons is still limited, and they lead to dissipation. To show the environment, Feynman and Vernon use the "bath of oscillator", Caldeira and Leggett follow this scenario. Here, the environment is a fairly large number of harmonic oscillators, and the mass, frequency, and coordinates are $m_{i}, \omega_{i}, x_{i}(t)$, where $i=1,2, \cdots, N$. They interact with the system (mass $M$, coordinate $q(t)$ ) in the potential field $V(q)$ and the external force $F$ ext $(t)$.

\section{Equation of Quantum Field for Space-Time Complexity}


Based on the above basic assumptions and the basic equations of quantum classical mechanics for the complexity of space-time, we will discuss the establishment of the basic equations of the quantum field theory for space-time complexity ( full view theory of quantum field ) through Wigner distribution as a nonlinear time-frequency transformation. From the information level, the basic equations (formula ( 215 ), ( 219 ) and ( 223 ) below) of the quantum field theory for space-time complexity ( full view theory of quantum field ) are a normalized scheme of ultra-synergy about classical field theory information and quantum field theory information.

In any system of quantum gravitational field, taking the limit $n \rightarrow \infty$ for the number of particles, where an operable model of the "environment" is the set of a large number of oscillators, or equivalently represented by scalar fields. The displacement coordinate (position) of matter fields is denoted as $\varphi_{x}(x, t)$, it interacts with the scalar field $\varphi(x, t)$ spreading in direction $y$ : $H_{\phi_{x}}^{\text {int }}=\Sigma \phi_{x} \frac{d \varphi}{d t}$; the order change (such as density difference, entropy change, information entropy change) coordinate of matter fields is $\varphi_{\sigma}(\sigma, t)$, it interacts with the scalar field $\varphi(x, t)$ spreading in direction $y: H_{\phi_{\sigma}}^{\mathrm{int}}=\Pi \phi_{\sigma} \frac{d \varphi}{d t}$. Introducing the full-view gauge parameter, there should be the following relation between the displacement coordinate $\varphi_{x}(x, t)$, order change (such as density difference, entropy change and information entropy change) coordinate $\varphi_{\sigma}(\sigma, t)$ and joint-varying coordinate $\varphi_{\gamma}(\gamma, t)$ of a matter field :

$$
\begin{aligned}
& H_{\phi_{\gamma}}^{\mathrm{int}}=\Pi \phi_{\gamma} \frac{d \varphi_{\gamma}}{d t}, \quad d \phi_{\gamma}=\frac{\partial \phi_{\gamma}}{\partial \phi_{x}} d \phi_{x}+\frac{\partial \phi_{\gamma}}{\partial \phi_{\sigma}} d \phi_{\sigma} ; \\
& H_{\phi_{r}}^{\mathrm{int}}=\Pi \phi_{r} \frac{d \varphi_{r}}{d t}, \quad d \phi_{r}=\frac{\partial \phi_{r}}{\partial \phi_{\gamma}} d \phi_{\gamma}-\frac{\partial \phi_{r}}{\partial \phi_{\sigma}} d \phi_{\sigma} ; \\
& H_{\phi_{\sigma}}^{\mathrm{int}}=\Pi \phi_{\sigma} \frac{d \varphi_{\sigma}}{d t}, \quad d \phi_{r}=\frac{\partial \phi_{\sigma}}{\partial \phi_{\gamma}} d \phi_{\gamma}-\frac{\partial \phi_{\sigma}}{\partial \phi_{r}} d \phi_{r} .
\end{aligned}
$$

Under the gauge, the ground state wave function of quantum gravitational system should have the form

$$
\begin{aligned}
& \Psi\left[h_{u, i j}, \phi_{c o m}\right]=N \int_{c} \delta g_{u, \mu v} \delta\left(\phi_{c o m}=\frac{\partial \phi_{c o m}}{\partial \phi_{m o v}} d \phi_{m o v}+\frac{\partial \phi_{c o m}}{\partial \phi_{d e v}} d \phi_{d e v}\right) \exp \left\{-I\left[g_{u, \mu v}, \phi_{c o m}\right]\right\} \\
& \Psi\left[h_{r, i j}, \phi_{m o v}\right]=N \int_{c} \delta g_{r, \mu v} \delta\left(\phi_{m o v}=\frac{\partial \phi_{m o v}}{\partial \phi_{c o m}} d \phi_{c o m}-\frac{\partial \phi_{m o v}}{\partial \phi_{d e v}} d \phi_{d e v}\right) \exp \left\{-I\left[g_{r, \mu v}, \phi_{m o v}\right]\right\} \\
& \Psi\left[h_{\zeta, i j}, \phi_{d e v}\right]=N \int_{c} \delta g_{\zeta, \mu v} \delta\left(\phi_{d e v}=\frac{\partial \phi_{d e v}}{\partial \phi_{c o m}} d \phi_{c o m}-\frac{\partial \phi_{d e v}}{\partial \phi_{m o v}} d \phi_{m o v}\right) \exp \left\{-I\left[g_{\zeta, \mu v}, \phi_{d e v}\right]\right\}
\end{aligned}
$$

where $I\left[g_{u, \mu v}, \varphi_{c o m}\right]$ is Euclidean joint-varying action quantity.

Not to set the restriction on temperature, we can set the master equation of fields satisfied by the field density matrix $\rho_{\phi, x}\left(\phi_{x}, \phi_{x}^{\prime}\right)$ in the expression of $\phi_{x}(x, t)$ : 


$$
\frac{d \rho_{\phi, x}}{d t}=-\frac{i}{\hbar}\left[H, \rho_{\phi, x}\right]-\omega\left(\phi_{x}-\phi_{x}^{\prime}\right)\left(\frac{d \rho_{\phi, x}}{d \phi_{x}}-\frac{d \rho_{\phi, x}}{d \phi_{x}^{\prime}}\right)-\frac{2 m \omega k T}{\hbar^{2}}\left(\phi_{x}-\phi_{x}^{\prime}\right)^{2} \rho_{\phi, x},
$$

and set the master equation of fields satisfied by the field density matrix $\rho_{\phi, \sigma}\left(\phi_{\sigma}, \phi_{\sigma}^{\prime}\right)$ in the expression of $\varphi_{\sigma}(\sigma, t)$ :

$$
\frac{d \rho_{\phi, \sigma}}{d t}=-\frac{i}{\hbar}\left[H, \rho_{\phi, \sigma}\right]-\mu\left(\phi_{\sigma}-\phi_{\sigma}^{\prime}\right)\left(\frac{d \rho_{\phi, \sigma}}{d \phi_{\sigma}}-\frac{d \rho_{\phi, \sigma}}{d \phi_{\sigma}^{\prime}}\right)-\frac{2 m \mu k T}{\hbar^{2}}\left(\phi_{\sigma}-\phi_{\sigma}^{\prime}\right)^{2} \rho_{\phi, \sigma},
$$

where, $H$ is the Hamilton quantity of fields, $\omega$ and $\mu$ are relaxation rates, and $T$ is the temperature of the field $\varphi$. On the one hand, $T$ relates to viscosity coefficient $\eta_{\varphi, x}$, while $\eta_{\varphi, x}$ depends on the coupling constant $\Sigma$ of fields and environment in $H_{\phi_{x}}^{\text {int }}$, i.e. $\omega=\eta_{\phi, x} / 2 m, \eta_{\phi, x}=\Sigma^{2} / 2$; on the other hand, relates to viscosity coefficient $\eta_{\varphi, \sigma}$, while $\eta_{\varphi, \sigma}$ depends on the coupling constant $\Pi$ of fields and environment in $H_{\phi_{\sigma}}^{\mathrm{int}}$, i.e. $\mu=\eta_{\phi, \sigma} / 2 m, \eta_{\phi, \sigma}=\Pi^{2} / 2$. In equation ( 204 ), the first item is the kinetic joint-varying of motion-like, and it is the inference of field equation. In equation ( 205 ), the first item is the kinetic joint-varying of evolution-like, and it is the extension of field equation. The second item is the dissipation effect of the environment, and the third item is the fluctuation causing the Brown motion, which is the root cause of the de-coherence.

By $\dot{\rho}_{\phi, \gamma}=\frac{\partial \rho_{\phi, \gamma}}{\partial \rho_{\phi, x}} \dot{\rho}_{\phi, x}+\frac{\partial \rho_{\phi, \gamma}}{\partial \rho_{\phi, \sigma}} \dot{\rho}_{\phi, \sigma}$, with ( 204 ) and ( 205 ), we can create the master equation of joint-varying matter fields satisfied by the particle density matrix $\rho_{\varphi, \gamma}\left(\varphi_{\gamma}, \varphi_{\gamma}^{\prime}\right)$ in the expression of $\varphi_{\gamma}(\gamma, t)$ :

$$
\begin{aligned}
\frac{d \rho_{\phi, \gamma}}{d t} & =\frac{\partial \rho_{\phi, \gamma}}{\partial \rho_{\phi, x}} \frac{d}{d t}\left\{-\frac{i}{\hbar}\left[H, \rho_{\phi, x}\right]-\omega\left(\phi_{x}-\phi_{x}^{\prime}\right)\left(\frac{d \rho_{\phi, x}}{d \phi_{x}}-\frac{d \rho_{\phi, x}}{d \phi_{x}^{\prime}}\right)-\frac{2 m \omega k T}{\hbar^{2}}\left(\phi_{x}-\phi_{x}^{\prime}\right)^{2} \rho_{\phi, x}\right\} \\
& +\frac{\partial \rho_{\phi, \gamma}}{\partial \rho_{\phi, \sigma}} \frac{d}{d t}\left\{-\frac{i}{\hbar}\left[H, \rho_{\phi, \sigma}\right]-\mu\left(\phi_{\sigma}-\phi_{\sigma}^{\prime}\right)\left(\frac{d \rho_{\phi, \sigma}}{d \phi_{\sigma}}-\frac{d \rho_{\sigma}}{d \phi_{\sigma}^{\prime}}\right)-\frac{2 m \mu k T}{\hbar^{2}}\left(\phi_{\sigma}-\phi_{\sigma}^{\prime}\right)^{2} \rho_{\phi, \sigma}\right\}
\end{aligned}
$$

By $\dot{\rho}_{\phi, x}=\frac{\partial \rho_{\phi, x}}{\partial \rho_{\phi, \gamma}} \dot{\rho}_{\phi, \gamma}-\frac{\partial \rho_{\phi, x}}{\partial \rho_{\phi, \sigma}} \dot{\rho}_{\phi, \sigma}$, with ( 204 ) and ( 205 ), we can give the following relation:

$$
\begin{gathered}
\frac{d \rho_{\phi, x}}{d t}=\frac{d \rho_{\phi, \gamma}}{d t} \frac{\partial}{\partial \rho_{\phi, \gamma}}\left\{-\frac{i}{\hbar}\left[H, \rho_{\phi, x}\right]-\omega\left(\phi_{x}-\phi_{x}^{\prime}\right)\left(\frac{d \rho_{\phi, x}}{d \phi_{x}}-\frac{d \rho_{\phi, x}}{d \phi_{x}^{\prime}}\right)-\frac{2 m \omega k T}{\hbar^{2}}\left(\phi_{x}-\phi_{x}^{\prime}\right)^{2} \rho_{\phi, x}\right\} \\
-\frac{d \rho_{\phi, \sigma}}{d t} \frac{\partial}{\partial \rho_{\phi, \sigma}}\left\{-\frac{i}{\hbar}\left[H, \rho_{\phi, x}\right]-\omega\left(\phi_{x}-\phi_{x}^{\prime}\right)\left(\frac{d \rho_{\phi, x}}{d \phi_{x}}-\frac{d \rho_{\phi, x}}{d \phi_{x}^{\prime}}\right)-\frac{2 m \omega k T}{\hbar^{2}}\left(\phi_{x}-\phi_{x}^{\prime}\right)^{2} \rho_{\phi, x}\right\} .
\end{gathered}
$$




$$
\begin{aligned}
& \text { By } \dot{\rho}_{\phi, \sigma}=\frac{\partial \rho_{\phi, \sigma}}{\partial \rho_{\phi, \gamma}} \dot{\rho}_{\phi, \gamma}-\frac{\partial \rho_{\phi, \sigma}}{\partial \rho_{\phi, x}} \dot{\rho}_{\phi, x} \text {, with ( } 204 \text { ) and ( } 205 \text { ), we can give the following relation : } \\
& \frac{d \rho_{\phi, \sigma}}{d t}=\frac{d \rho_{\phi, \sigma}}{d t} \frac{\partial}{\partial \rho_{\phi, \gamma}}\left\{-\frac{i}{\hbar}\left[H, \rho_{\phi, \sigma}\right]-\mu\left(\phi_{\sigma}-\phi_{\sigma}^{\prime}\right)\left(\frac{d \rho_{\phi, \sigma}}{d \phi_{\sigma}}-\frac{d \rho_{\phi, \sigma}}{d \phi_{\sigma}^{\prime}}\right)-\frac{2 m \mu k T}{\hbar^{2}}\left(\phi_{\sigma}-\phi_{\sigma}^{\prime}\right)^{2} \rho_{\phi, \sigma}\right\} \\
& -\frac{d \rho_{\phi, x}}{d t} \frac{\partial}{\partial \rho_{\phi, x}}\left\{-\frac{i}{\hbar}\left[H, \rho_{\phi, \sigma}\right]-\mu\left(\phi_{\sigma}-\phi_{\sigma}^{\prime}\right)\left(\frac{d \rho_{\phi, \sigma}}{d \phi_{\sigma}}-\frac{d \rho_{\phi, \sigma}}{d \phi_{\sigma}^{\prime}}\right)-\frac{2 m \mu k T}{\hbar^{2}}\left(\phi_{\sigma}-\phi_{\sigma}^{\prime}\right)^{2} \rho_{\phi, \sigma}\right\}
\end{aligned}
$$

For the joint-varying gravitational field, the coherent super-positions of two Gauss wave packets are considered:

$$
X\left(\phi_{x}\right)=X^{+}\left(\phi_{x}\right)+X^{-}\left(\phi_{x}\right), \quad \Theta\left(\phi_{\sigma}\right)=\Theta^{+}\left(\phi_{\sigma}\right)+\Theta^{-}\left(\phi_{\sigma}\right),
$$

where the width of wave packet are $\delta_{\varphi, x}$ and $\delta_{\varphi, \sigma}$, the distance that they are away from the center are respectively $\Delta \varphi_{x}$ and $\Delta \varphi_{\sigma}, \Delta \varphi_{x} \ll<\delta_{\varphi, x}, \Delta \varphi_{\sigma} \ll<\delta_{\varphi, \sigma}$. There are 4 peaks on plane $\left(\varphi_{x}, \varphi^{\prime}{ }_{x}\right)$ in the density matrix $\rho_{\varphi, x}\left(\varphi_{x}, \varphi^{\prime}{ }_{x}\right)=X\left(\varphi_{x}\right) X^{*}\left(\varphi^{\prime}{ }_{x}\right)$, where two come from the diagonal element (the position $\varphi_{x}=\varphi^{\prime}{ }_{x}$ of the peak), the other two are from the non diagonal element $\left(\varphi_{x}=-\varphi^{\prime}{ }_{x}\right)$. The last item of master equation ( 204$)$ is proportional to $\left(\varphi_{x}-\varphi^{\prime}{ }_{x}\right)^{2}$. It has little effect on the diagonal element, but on the non diagonal element becomes the factor causing the attenuation. The decay rate is written directly from the equation: $\tau_{D, x}^{-1} \approx 2 \omega \frac{m k T\left(\Delta \phi_{x}\right)^{2}}{\hbar^{2}}, \tau_{D, \sigma}^{-1} \approx 2 \mu \frac{m k T\left(\Delta \phi_{\sigma}\right)^{2}}{\hbar^{2}}$. De-coherence time $\tau_{D, x}$ and $\tau_{D, \sigma}$ are respectively

$$
\tau_{D, x} \approx \tau_{R} \frac{\hbar^{2}}{2 m k T\left(\Delta \phi_{x}\right)^{2}}=\tau_{R}\left(\frac{\lambda_{T}}{\Delta \phi_{x}}\right)^{2}, \quad \tau_{D, \sigma} \approx \tau_{R} \frac{\hbar^{2}}{2 m k T\left(\Delta \phi_{\sigma}\right)^{2}}=\tau_{R}\left(\frac{\lambda_{T}}{\Delta \phi_{\sigma}}\right)^{2},
$$

Because $\lambda_{T}=\frac{\hbar}{\sqrt{2 m k T}}$ is the wavelength of thermal de Broglie, $\tau_{R, x}=\frac{1}{\omega}$ and $\tau_{R, \sigma}=\frac{1}{\mu}$ are relaxation time, $\tau_{D, x}$ can be written $\tau_{D, x}=\tau_{R, x}\left(\frac{\lambda_{T}}{\Delta \phi_{x}}\right)^{2}, \tau_{D, \sigma}$ can be written $\tau_{D, \sigma}=\tau_{R, \sigma}\left(\frac{\lambda_{T}}{\Delta \phi_{\sigma}}\right)^{2}$.

We know that quantum field theory is expressed in Hilbert spaces, and classical field theory is expressed in phase space. Under the full-view gauge put forth in this series of papers, by the the following relation:

$$
\begin{aligned}
& d W_{\phi, \gamma}\left(\phi_{\gamma}, p\right)=\Delta W_{\phi, x}\left(\phi_{x}, p\right) \frac{\partial W_{\phi, \gamma}\left(\phi_{\gamma}, p\right)}{\partial W_{\phi, x}}+\Delta W_{\phi, \sigma}\left(\phi_{\sigma}, p\right) \frac{\partial W_{\phi, \gamma}\left(\phi_{\gamma}, p\right)}{\partial W_{\phi, \sigma}}, \\
& d W_{\phi, x}\left(\phi_{x}, p\right)=\Delta W_{\phi, \gamma}\left(\phi_{\gamma}, p\right) \frac{\partial W_{\phi, x}\left(\phi_{x}, p\right)}{\partial W_{\phi, \gamma}}-\Delta W_{\phi, \sigma}\left(\phi_{\sigma}, p\right) \frac{\partial W_{\phi, x}\left(\phi_{x}, p\right)}{\partial W_{\phi, \sigma}},
\end{aligned}
$$




$$
d W_{\phi, \sigma}\left(\phi_{\sigma}, p\right)=\Delta W_{\phi, \gamma}\left(\phi_{\gamma}, p\right) \frac{\partial W_{\phi, \sigma}\left(\phi_{\sigma}, p\right)}{\partial W_{\phi, \gamma}}-\Delta W_{\phi, x}\left(\phi_{x}, p\right) \frac{\partial W_{\phi, \sigma}\left(\phi_{\sigma}, p\right)}{\partial W_{\phi, x}} .
$$

We can use the Wigner transform of gravitational field function, set the following relation between the quantum gravitational field and its classical limit :

$$
\begin{gathered}
W_{\phi, x}\left(\phi_{x}, p\right)=\frac{1}{2 \pi \hbar} \int_{-\infty}^{\infty} e^{i p y / \hbar} \psi_{\phi, x}^{*}\left(\phi_{x}+\frac{y}{2}\right) \psi_{\phi, x}\left(\phi_{x}-\frac{y}{2}\right) d y, \\
W_{\phi, \sigma}\left(\phi_{\sigma}, p\right)=\frac{1}{2 \pi \hbar} \int_{-\infty}^{\infty} e^{i p y / \hbar} \Psi_{\phi, \sigma}^{*}\left(\phi_{\sigma}+\frac{y}{2}\right) \Psi_{\sigma}\left(\phi_{\sigma}-\frac{y}{2}\right) d y,
\end{gathered}
$$

Wigner distributions $W_{\varphi, x}\left(\varphi_{x}, p\right)$ and $W_{\varphi, \sigma}\left(\varphi_{\sigma}, p\right)$ are real function, but may be negative. Therefore, in general, it cannot be interpreted as a distribution function. However, if $W_{\varphi, x}\left(\varphi_{x}, p\right)$ and $W_{\varphi, \sigma}\left(\varphi_{\sigma}, p\right)$ are integral respectively to $p$, then

$$
\begin{gathered}
\int_{-\infty}^{\infty} W_{\phi, x}\left(\phi_{x}, p\right) d p=\int_{-\infty}^{\infty} \delta(y) \Psi_{\phi, x}^{*}\left(\phi_{x}+\frac{y}{2}\right) \Psi_{\phi, x}\left(\phi_{x}-\frac{y}{2}\right) d y=\Psi_{\phi, x}^{*}\left(\phi_{x}\right) \Psi_{\phi, x}\left(\phi_{x}\right) \\
\int_{-\infty}^{\infty} W_{\phi, \sigma}\left(\phi_{\sigma}, p\right) d p=\int_{-\infty}^{\infty} \delta(y) \Psi_{\phi, \sigma}^{*}\left(\phi_{\sigma}+\frac{y}{2}\right) \Psi_{\phi, \sigma}\left(\phi_{\sigma}-\frac{y}{2}\right) d y=\Psi_{\phi, \sigma}^{*}\left(\phi_{\sigma}\right) \Psi_{\phi, \sigma}\left(\phi_{\sigma}\right)
\end{gathered}
$$

are respectively the distribution of $\varphi_{x}$ and the distribution of $\varphi_{\sigma}$.

Give the Fourier transform of $\Psi_{\phi, x}: \varphi_{\phi, x}(p)=\frac{1}{\sqrt{2 \pi \hbar}} \int_{-\infty}^{\infty} e^{-i p \xi_{x} / \hbar} \Psi_{\phi, x}\left(\xi_{x}\right) d \xi_{x}$, the distribution of $p$ is

$$
\varphi_{\phi, x}^{*}(p) \varphi_{\phi, x}(p)=\frac{1}{2 \pi \hbar} \int_{-\infty}^{\infty} e^{i p\left(\lambda_{x}-\xi_{x}\right) / \hbar} \Psi_{\phi, x}^{*}\left(\lambda_{x}\right) \Psi_{\phi, x}\left(\xi_{x}\right) d \xi_{x} d \lambda_{x}
$$

Let $\lambda_{x}=\phi_{x}+\frac{y}{2}, \xi_{x}=\phi_{x}-\frac{y}{2}$, there is

$$
\varphi_{\phi, x}^{*}(p) \varphi_{\phi, x}(p)=\frac{1}{2 \pi \hbar} \int_{-\infty}^{\infty} e^{i p y / \hbar} \Psi_{\phi, x}^{*}\left(\phi_{x}+\frac{y}{2}\right) \Psi_{\phi, x}\left(\phi_{x}-\frac{y}{2}\right) d \phi_{x} d y=\int_{-\infty}^{\infty} W_{\phi, x}\left(\phi_{x}, p\right) d \phi_{x}
$$

i.e. the integral of $W_{\phi, x}\left(\phi_{x}, p\right)$ to $\phi_{x}$ gives the distribution of $p$.

Give the Fourier transform of $\Psi_{\phi, \sigma}: \varphi_{\phi, \sigma}(p)=\frac{1}{\sqrt{2 \pi \hbar}} \int_{-\infty}^{\infty} e^{-i p \xi_{\sigma} / \hbar} \Psi_{\phi, \sigma}\left(\xi_{\sigma}\right) d \xi_{\sigma}$, the distribution of $p$ is

$$
\varphi_{\phi, \sigma}^{*}(p) \varphi_{\phi, \sigma}(p)=\frac{1}{2 \pi \hbar} \int_{-\infty}^{\infty} e^{i p\left(\lambda_{\sigma}-\xi_{\sigma}\right) / \hbar} \Psi_{\phi, \sigma}^{*}\left(\lambda_{\sigma}\right) \Psi_{\phi, \sigma}\left(\xi_{\sigma}\right) d \xi_{\sigma} d \lambda_{\sigma}
$$

Let $\lambda_{\sigma}=\phi_{x}+\frac{y}{2}, \xi_{\sigma}=\phi_{x}-\frac{y}{2}$, there is 


$$
\varphi_{\phi, \sigma}^{*}(p) \varphi_{\phi, \sigma}(p)=\frac{1}{2 \pi \hbar} \int_{-\infty}^{\infty} e^{i p y / \hbar} \Psi_{\phi, \sigma}^{*}\left(\phi_{\sigma}+\frac{y}{2}\right) \Psi_{\phi, \sigma}\left(\phi_{\sigma}-\frac{y}{2}\right) d \phi_{\sigma} d y=\int_{-\infty}^{\infty} W_{\phi, \sigma}\left(\phi_{\sigma}, p\right) d \phi_{\sigma}
$$

i.e. the integral of $W_{\phi, \sigma}\left(\phi_{\sigma}, p\right)$ to $\phi_{\sigma}$ gives the distribution of $p$.

For the displacing wave packet

$$
\psi_{\phi, x}\left(\phi_{x}\right) \approx \exp \left[-\left[\phi_{x}-\phi_{x, 0}=\left(\phi_{\gamma}-\phi_{\gamma, 0}\right) \frac{\partial \phi_{x}}{\partial \phi_{\gamma}}-\left(\phi_{\sigma}-\phi_{\sigma, 0}\right) \frac{\partial \phi_{x}}{\partial \phi_{\sigma}}\right]^{2} / \delta^{2}+i \frac{p_{0} \phi_{x}}{\hbar}\right]
$$

of minimum uncertainty, Wigner distribution is

$$
\psi_{\phi, x}\left(\phi_{x}, p\right)=\frac{1}{\pi \hbar} \exp \left[-\left[\phi_{x}-\phi_{x, 0}=\left(\phi_{\gamma}-\phi_{\gamma, 0}\right) \frac{\partial \phi_{x}}{\partial \phi_{\gamma}}-\left(\phi_{\sigma}-\phi_{\sigma, 0}\right) \frac{\partial \phi_{x}}{\partial \phi_{\sigma}}\right]^{2} / \delta^{2}-\frac{\left(p-p_{0}\right)^{2} \delta^{2}}{\hbar^{2}}\right],
$$

where $\varphi_{x}$ and $p$ obey Gauss distribution, and meet the minimum uncertainty relation, it shows that this $\Psi_{\phi, x}$ is the nearest simulation of the motion-like wave function (the vector in Hilbert space) of quantum gravitational field for the classical equation of a motion-like gravitational field. Wigner distribution can be extended to the density matrix

$$
W_{\phi, x}\left(\phi_{x}, p\right)=\frac{1}{2 \pi \hbar} \int_{-\infty}^{\infty} e^{i p y / \hbar} \rho_{\phi, x}\left(\phi_{x}-\frac{y}{2}, \phi_{x}+\frac{y}{2}\right) d y .
$$

For the coherent superposition $X=X^{+}+X^{-}$of two Gauss wave packets given in expression ( 209 ), its Wigner distribution is

$$
W_{\phi, x} \approx \frac{W_{\phi, x}^{+}+W_{\phi, x}^{-}}{2}+\frac{1}{\pi \hbar} \exp \left[-\frac{1}{\hbar^{2}} p^{2} \delta^{2}-\frac{\phi_{x}^{2}}{\delta^{2}}\right] \cos \left(p \frac{\Delta \phi_{x}}{\hbar}\right)
$$

where $W_{\phi, x}^{+}$and $W_{\phi, x}^{-}$are the Wigner distribution of $X^{+}$and $X^{-}$. Because of the oscillatory behavior of $W_{\phi, x}$, it can not be interpreted as the distribution of phase space. The motion equation of $W_{\phi, x}$ can be given by the master equation ( 204$)$ for the density matrix $\rho_{x}\left(\phi_{x}, \phi_{x}^{\prime}\right)$ of fields to meet :

$$
\frac{d W_{\phi, x}}{d t}=\left(-\frac{p}{m} \frac{\partial W_{\phi, x}}{\partial \phi_{x}}+\frac{\partial V}{\partial \phi_{x}} \frac{\partial W_{\phi, x}}{\partial p}\right)+2 \omega \frac{\partial\left(p W_{\phi, x}\right)}{\partial p}+D \frac{\partial^{2} W_{\phi, x}}{\partial p^{2}},
$$

where $V$ is potential energy, $D_{x}=2 m \omega k T$, the second item is the damping term, the third item is the diffusion of $W_{\phi, x}$ in the momentum space, with the diffusion coefficient as $D_{x}$. The first item in the bracket of equation ( 215 ) is the classic Poisson bracket : 


$$
\left[H, W_{\phi, x}\right]_{P o i s s o n}=\frac{\partial H}{\partial \phi_{x}} \frac{\partial W_{\phi, x}}{\partial p}-\frac{\partial H}{\partial p} \frac{\partial W_{\phi, x}}{\partial \phi_{x}}
$$

it is because of $\frac{\partial H}{\partial \phi_{x}}=\frac{\partial V}{\partial \phi_{x}}$ and $\frac{\partial H}{\partial p}=\dot{\phi}_{x}=\frac{p}{m}$. The Liouville form of classical dynamics in phase space for harmonic oscillator is derived from quantum dynamics. For the general displacement system of field, in the right of equation ( 215 ) there shall be also the quantum correction with the order of $O$ ( $\hbar$ ), the 2nd item is damping, the 3 rd item is the diffusion of $W_{\phi, x}$ in momentum space, with the diffusion coefficient as $D_{x}$. In fact, the effect of the diffusion term is simple. Since the oscillation term $\cos \left(p \frac{\Delta \phi_{x}}{\hbar}\right)$ in equation ( 214$)$ is an eigen-function of $\frac{\partial^{2}}{\partial p^{2}}$, the diffusion term tends to decrease the oscillation term, and the decay rate is $\tau_{D, x}^{-1}=2 m \omega k T\left(\frac{\Delta \phi_{x}}{\hbar}\right)^{2}$.

For the evolution wave packet

$$
\psi_{\phi, \sigma}\left(\phi_{\sigma}\right) \approx \exp \left[-\left[\phi_{\sigma}-\phi_{\sigma, 0}=\left(\phi_{\gamma}-\phi_{\gamma, 0}\right) \frac{\partial \phi_{\sigma}}{\partial \phi_{\gamma}}-\left(\phi_{x}-\phi_{x, 0}\right) \frac{\partial \phi_{\sigma}}{\partial \phi_{x}}\right]^{2} / \delta^{2}+i \frac{p_{0} \phi_{\sigma}}{\hbar}\right]
$$

of minimum uncertainty, Wigner distribution is

$$
\psi_{\phi, \sigma}\left(\phi_{\sigma}, p\right)=\frac{1}{\pi \hbar} \exp \left[-\left[\phi_{\sigma}-\phi_{\sigma, 0}=\left(\phi_{\gamma}-\phi_{\gamma, 0}\right) \frac{\partial \phi_{\sigma}}{\partial \phi_{\gamma}}-\left(\phi_{x}-\phi_{x, 0}\right) \frac{\partial \phi_{\sigma}}{\partial \phi_{x}}\right]^{2} / \delta^{2}-\frac{\left(p-p_{0}\right)^{2} \delta^{2}}{\hbar^{2}}\right],
$$

where $\varphi_{\sigma}$ and $p$ obey Gauss distribution, and meet the minimum uncertainty relation, it shows that this $\Psi_{\phi, \sigma}$ is the nearest simulation of the evolution-like wave function (the vector in Hilbert space) of quantum gravitational field for the classical evolution of an evolution-like gravitational field. Wigner distribution can be extended to the density matrix

$$
W_{\phi, \sigma}\left(\phi_{\sigma}, p\right)=\frac{1}{2 \pi \hbar} \int_{-\infty}^{\infty} e^{i p y / \hbar} \rho_{\phi, \sigma}\left(\phi_{\sigma}-\frac{y}{2}, \phi_{\sigma}+\frac{y}{2}\right) d y .
$$

For the coherent superposition $\Xi=\Xi^{+}+\Xi^{-}$of two Gauss wave packets given in expression ( 209 ), its Wigner distribution is

$$
W_{\sigma} \approx \frac{W_{\sigma}^{+}+W_{\sigma}^{-}}{2}+\frac{1}{\pi \hbar} \exp \left[-\frac{1}{\hbar^{2}} p^{2} \delta^{2}-\frac{\sigma^{2}}{\delta^{2}}\right] \cos \left(p \frac{\Delta \sigma}{\hbar}\right),
$$

where $W_{\phi, \sigma}^{+}$and $W_{\phi, \sigma}^{-}$are the Wigner distribution of $\Xi^{+}$and $\Xi^{-}$. Because of the oscillatory behavior 
of $W_{\phi, \sigma}$, it can not be interpreted as the distribution of phase space. The evolution equation of $W_{\phi, \sigma}$ can be given by the master equation ( 205 ) for the density matrix $\rho_{\phi, \sigma}\left(\phi_{\sigma}, \phi_{\sigma}^{\prime}\right)$ of fields to meet :

$$
\frac{d W_{\phi, \sigma}}{d t}=\left(-\frac{p}{m} \frac{\partial W_{\phi, \sigma}}{\partial \phi_{\sigma}}+\frac{\partial V}{\partial \phi_{\sigma}} \frac{\partial W_{\phi, \sigma}}{\partial p}\right)+2 \omega \frac{\partial\left(p W_{\phi, \sigma}\right)}{\partial p}+D \frac{\partial^{2} W_{\phi, \sigma}}{\partial p^{2}}
$$

where $V$ is potential energy, $D_{\sigma}=2 m \mu k T$, the second item is the damping term, the third item is the diffusion of $W_{\phi, \sigma}$ in the momentum space, with the diffusion coefficient as $D_{\sigma}$. The first item in the bracket of equation ( 219 ) is the classic Poisson bracket :

$$
\left[H, W_{\phi, \sigma}\right]_{P o i s s o n}=\frac{\partial H}{\partial \phi_{\sigma}} \frac{\partial W_{\phi, \sigma}}{\partial p}-\frac{\partial H}{\partial p} \frac{\partial W_{\phi, \sigma}}{\partial \phi_{\sigma}},
$$

it is because of $\frac{\partial H}{\partial \phi_{\sigma}}=\frac{\partial V}{\partial \phi_{\sigma}}$ and $\frac{\partial H}{\partial p}=\dot{\phi}_{\sigma}=\frac{p}{m}$. For the classical gravitational field, in the right of equation ( 219 ) there shall be also the quantum correction with the order of $O(\hbar)$, the 2 nd item is damping, the 3 rd item is the diffusion of $W_{\phi, \sigma}$ in momentum space, with the diffusion coefficient as $D_{\sigma}$. In fact, the effect of the diffusion term is simple. Since the oscillation term $\cos \left(p \frac{\Delta \phi_{\sigma}}{\hbar}\right)$ in equation ( 218 ) is an eigen-function of $\frac{\partial^{2}}{\partial p^{2}}$, the diffusion term tends to decrease the oscillation term, and the decay rate is $\tau_{D, \sigma}^{-1}=2 m \mu k T\left(\frac{\Delta \phi_{\sigma}}{\hbar}\right)^{2}$.

For the joint-varying wave packet

$$
\psi_{\phi, \gamma}\left(\phi_{\gamma}\right) \approx \exp \left[-\left[\phi_{\gamma}-\phi_{\gamma, 0}=\left(\phi_{x}-\phi_{x, 0}\right) \frac{\partial \phi_{\gamma}}{\partial \phi_{x}}+\left(\phi_{\sigma}-\phi_{\sigma, 0}\right) \frac{\partial \phi_{\gamma}}{\partial \phi_{\sigma}}\right]^{2} / \delta^{2}+i \frac{p_{0} \phi_{\gamma}}{\hbar}\right]
$$

of minimum uncertainty, Wigner distribution

$$
\psi_{\phi, \gamma}\left(\phi_{\gamma}, p\right)=\frac{1}{\pi \hbar} \exp \left[-\left[\phi_{\gamma}-\phi_{\gamma, 0}=\left(\phi_{x}-\phi_{x, 0}\right) \frac{\partial \phi_{\gamma}}{\partial \phi_{x}}+\left(\phi_{\sigma}-\phi_{\sigma, 0}\right) \frac{\partial \phi_{\gamma}}{\partial \phi_{\sigma}}\right]^{2} / \delta^{2}-\frac{\left(p-p_{0}\right)^{2} \delta^{2}}{\hbar^{2}}\right],
$$

where $\varphi_{\gamma}$ and $p$ obey Gauss distribution, and meet the minimum uncertainty relation, it shows that this $\Psi_{\phi, \gamma}$ is the nearest simulation of the joint-varying wave function (the vector in Hilbert space) of quantum gravitational field for the classical joint-varying equation of a gravitational field (a point in 
phase space). Wigner joint-distribution can be extended to the density matrix

$$
W_{\phi, \gamma}\left(\phi_{\gamma}, p\right)=\frac{1}{2 \pi \hbar} \int_{-\infty}^{\infty} e^{i p y / \hbar} \rho_{\phi, \gamma}\left(\phi_{\gamma}-\frac{y}{2}, \phi_{\gamma}+\frac{y}{2}\right) d y .
$$

For the coherent superposition $X\left(\phi_{x}\right)=X^{+}\left(\phi_{x}\right)+X^{-}\left(\phi_{x}\right)$ and $\Theta\left(\phi_{\sigma}\right)=\Theta^{+}\left(\phi_{\sigma}\right)+\Theta^{-}\left(\phi_{\sigma}\right)$ of two Gauss wave packets given in expression ( 209 ), theirs Wigner joint-distribution is

$$
W_{\phi, \gamma} \approx \frac{W_{\phi, \gamma}^{+}+W_{\phi, \gamma}^{-}}{2}+\frac{1}{\pi \hbar} \exp \left[-\frac{1}{\hbar^{2}} p^{2} \delta^{2}-\frac{\phi_{\gamma}^{2}}{\delta^{2}}\right] \cos \left(p \frac{\Delta \phi_{\gamma}}{\hbar}\right)
$$

where $W_{\phi, \gamma}^{+}$and $W_{\phi, \gamma}^{-}$are the Wigner joint-distribution. The joint-varying equation (as the basic equation of quantum complexity mechanics for space-time complexity) of $W_{\phi, \gamma}$ can be given by the master equation ( 206 ) for the density matrix $\rho_{\phi, \gamma}\left(\phi_{\gamma}, \phi_{\gamma}^{\prime}\right)$ of fields to meet :

$$
\begin{aligned}
\frac{d W_{\phi, \gamma}}{d t} & =\frac{d \rho_{\phi, x}}{d t} \frac{\partial}{\partial \rho_{\phi, x}} \int d t\left\{\left(-\frac{p}{m} \frac{\partial W_{\phi, x}}{\partial \phi_{x}}+\frac{\partial V}{\partial \phi_{x}} \frac{\partial W_{\phi, x}}{\partial p}\right)+2 \omega \frac{\partial\left(p W_{\phi, x}\right)}{\partial p}+D \frac{\partial^{2} W_{\phi, x}}{\partial p^{2}}\right\} \\
& +\frac{d \rho_{\phi, \sigma}}{d t} \frac{\partial}{\partial \rho_{\phi, \sigma}} \int d t\left\{\left(-\frac{p}{m} \frac{\partial W_{\phi, x}}{\partial \phi_{x}}+\frac{\partial V}{\partial \phi_{x}} \frac{\partial W_{\phi, x}}{\partial p}\right)+2 \omega \frac{\partial\left(p W_{\phi, x}\right)}{\partial p}+D \frac{\partial^{2} W_{\phi, x}}{\partial p^{2}}\right\}
\end{aligned}
$$

where $V$ is potential energy. The first item in the bracket of equation ( 223 ) is the classic Poisson bracket under the full-view gauge :

$$
\left[H, W_{\phi, \gamma}\right]_{\text {Poisson }}=\frac{\partial H}{\partial \phi_{\gamma}} \frac{\partial W_{\phi, \gamma}}{p}-\frac{\partial H}{\partial p} \frac{\partial W_{\phi, \gamma}}{\partial \phi_{\gamma}},
$$

it is because of $\frac{\partial H}{\partial \phi_{\gamma}}=\frac{\partial V}{\partial \phi_{\gamma}}$ and $\frac{\partial H}{\partial p}=\dot{\phi}_{\gamma}=\frac{p}{m}$. For the general joint-varying field system, in the right of equation ( 223 ) there shall be also the quantum correction with the order of $O(\hbar)$, the 2 nd item is damping, the 3 rd item is the diffusion of $W_{\phi, \gamma}$ in momentum space, with the diffusion coefficient as $D$ $\gamma$. Since the oscillation term $\cos \left(p \frac{\Delta \gamma}{\hbar}\right)$ in equation ( 222 ) is an eigen-function of $\frac{\partial^{2}}{\partial p^{2}}$, the diffusion term tends to decrease the oscillation term, and the decay rate is $\tau_{D, \gamma}^{-1}=2 m \aleph k T\left(\frac{\Delta \phi_{\gamma}}{\hbar}\right)^{2}, \aleph$ is related to relaxation rates $\omega$ and $\mu$.

\section{Summary of This Series of Studies}


To solve a series of major problems, such as the singularity before the big bang, the inflation of the early universe and the accelerating expansion of the present universe, this series of studies start with super gravity, dark energy and dark matter, go beyond the surface of a lot of observation data, deeply and systematically reveal the arche-internal mechanism of all things, establish the arche-unity of physics, and give a natural and reasonable explanation.

In this regard, we have considered noncommutative spacetime ${ }^{[31]-[34][40][89]}$, introduced ultimate super- symmetry and arche-conjugation, and then introduced holographic quantum unity and ultra-synergy. We expect that the closer it is to Planck scale, the stronger its conventional quantum effect, non commutative quantum effect, arche-conjugate effect, co-frequency resonance effect, holographic quantum unity effect and ultra-synergy effect are.

Now, for a series of major difficult problems, such as the singularity before the big bang, the inflation of the early universe and the accelerating expansion of the present universe, we can sum up all the previous research results of this series, and give the corresponding analytical framework of natural mechanism. In general, the analytical framework of this mechanism consists of the following aspects: non-commutative space-time; ultimate symmetry; arche-conjugation; arche-pulsation; quantum hedge -unification; holographic quantum unity; ultra-synergy.

The arche-unified physical analysis framework of natural mechanism is shown in Figure 11 (Li Zongcheng, 2019).

The mechanism of holographic quantum unified interaction revealed in this series shows that: the dominant role of supergravity in high dimension (d5-d11) space-time corresponds to the dominant role of superrepulsion in low dimension ( $\mathrm{d} 1-\mathrm{d} 4)$ space-time; the dominant role of high dimensional supergravity and quantum gravity in small scale (close to Planck space-time) corresponds to the dominant role of high dimensional superrepulsion and quantum repulsion in large scale (greater than Planck space-time).

All the previous analysis and discussion in this series can get the following results:

( $a$ ) at the level of quantum gravity, the interaction between high-dimensional supergravity and high-dimensional superrepulsion, as well as the interaction between quantum gravity and quantum repulsion, is a process that happens all the time and everywhere, which can be called the quantum pulsation process of the arche-conjugation.

( $b$ ) although dark energy and dark matter are introduced to analyze and explain the accelerated expansion of the universe, they are essentially the phenomena of quantum repulsion and quantum gravity.

( $c$ ) quantum gravity doesn't only exist in the early universe and all kinds of the later black holes. In fact, they interact with quantum repulsion all the time and everywhere, and exist in our universe in the form of the interaction between dark energy and dark matter.

(1) noncommutative time and space

For supergravity, dark energy and dark matter, we enter into supersymmetric space-time, quantum gravitational space-time (or Planck space-time), non-commutative quantum space-time, and will introduce the arche-pulsating space-time, which always exists and determines to affect the current and future world.

One of the characteristics of quantum mechanics is that in this theory coordinates and coordinates, momentum and momentum are commutative, while the relationship between coordinates and their 
conjugate momentum is non commutative. Some researches on string theory show that the coordinates are not commutative when they are close to Planck scale, and its relation satisfies

$$
\left[\tilde{x}^{\mu}, \tilde{x}^{v}\right]=i \alpha M^{\mu v}
$$

here $\alpha \in R, M^{\mu v}$ is an anti-symmetric matrix, $\tilde{x}$ is a noncommutative coordinate. In this way, common quantum mechanics becomes noncommutative quantum mechanics. In order to ensure that the system

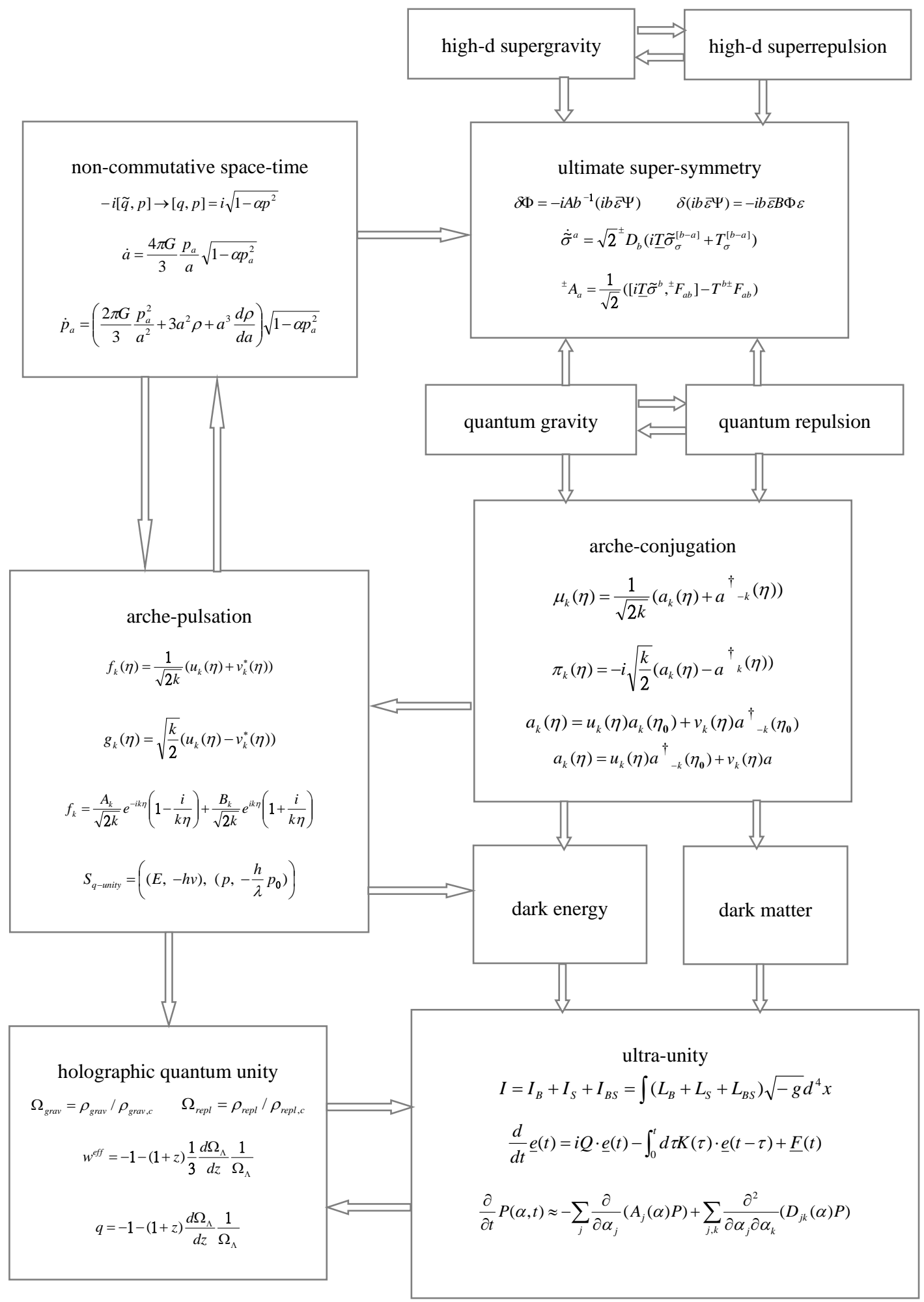




\section{Figure 11 Arche-Unified Physical Analysis Framework of Natural Mechanism}

has rotational symmetry, it is necessary to have $\left[p^{\mu}, p^{v}\right]=0$, the noncommutative relationship between coordinates and momentum becomes

$$
[\tilde{x}, p]=i \sqrt{1-\alpha p^{2}}
$$

When $\alpha \rightarrow 0$, the theory goes back to quantum mechanics. In 2009, M. Battisti considered the non commutative geometry in Synder space ${ }^{[71]}$, and obtained the modified Friedmann equation through the quantum classical correspondence. The general process is relatively simple: under the classical limit, the commutation relation of operators should return to the form of Poisson bracket:

$$
-i[\tilde{q}, p] \rightarrow[q, p]=i \sqrt{1-\alpha p^{2}}
$$

Therefore, the relation a is satisfied for the commutative scale factor $a$ and its conjugate momentum $p_{a}$, where

$$
\begin{gathered}
\dot{a}=\frac{4 \pi G}{3} \frac{p_{a}}{a} \sqrt{1-\alpha p_{a}^{2}} \\
\dot{p}_{a}=\left(\frac{2 \pi G}{3} \frac{p_{a}^{2}}{a^{2}}+3 a^{2} \rho+a^{3} \frac{d \rho}{d a}\right) \sqrt{1-\alpha p_{a}^{2}}
\end{gathered}
$$

By the above two formulas and the Hamiltonian constraint $H_{N C} \approx 0$, we get the background evolution equation:

$$
H^{2}=\frac{8 \pi G}{3} \rho\left(1-\operatorname{sgn} a \frac{\rho}{\rho_{c}}\right)
$$

where the critical density is $\rho_{c}=\rho_{p}(2 \pi G / 3|\alpha|)$. When $\alpha \rightarrow 0, \rho_{c} \rightarrow \infty$ returns to the classical case. In the case of $\alpha>0$, Friedmann equation has a rebound solution.

( 2 ) Ultimate Supersymmetry

As a mathematical method, supersymmetry has very rich physical significance, which is related to superfield, hyperspace, super transformation and so on. In superfield, such as scalar superfield $\Phi(x, \theta)$, Bose field $\varphi$ (including all kinds of scalar field, vector field, tensor field, etc.) and Fermi field $\psi$ are included. There are all kinds of super gauge transformations between the two fields ${ }^{[119]-[121]}$.

Now, in the super unifying force field $\Omega(X, \Theta)$ introduced here, we consider the gravitational field (especially the high-dimensional super gravitational field including Fermi field) $\Phi$ on the one hand, and the repulsive field (especially the high-dimensional super repulsive field including Bose field) $\Psi$ on the other hand. All kinds of supernormal transformations between gravitational field and repulsive field can be summarized as follows: 


$$
\delta \Phi=A \bar{\varepsilon} \Psi, \quad \delta \Psi=-B \Phi \varepsilon,
$$

where, $\varepsilon$ is the rotation angle of the hyperparticle, as the major rotation of the anti commutation.

Suppose the multiplicity of sparticle is $\Omega=\left(\begin{array}{c}\Phi \\ i b \vec{\varepsilon} \Psi\end{array}\right)$, formula ( 229 ) becomes a

$$
\delta \Phi=-i A b^{-1}(i b \bar{\varepsilon} \Psi), \quad \delta(i b \bar{\varepsilon} \Psi)=-i b \bar{\varepsilon} B \Phi \varepsilon,
$$

so

$$
\left(\begin{array}{c}
\Phi^{\prime} \\
i b \vec{\varepsilon} \Psi^{\prime}
\end{array}\right)=\left(\begin{array}{cc}
1 & -i A b^{-1} \\
-i b \dot{\varepsilon} B \varepsilon & 1
\end{array}\right)\left(\begin{array}{c}
\Phi \\
i b \vec{\varepsilon} \Psi
\end{array}\right)
$$

here $\varepsilon$ is commutative to the repulsive field $\Phi$ and anti-commutative to the gravitational field $\Psi$, then

$$
\alpha_{\mu \nu}=\left(\begin{array}{cc}
1 & -i A b^{-1} \\
-i b \bar{\varepsilon} B \varepsilon & 1
\end{array}\right)=\left(\begin{array}{ll}
1 & 0 \\
0 & 1
\end{array}\right)-i\left(\begin{array}{cc}
0 & A b^{-1} \\
b \bar{\varepsilon} B \varepsilon & 0
\end{array}\right) .
$$

The Lagrangian of super unified field is:

$$
L=\frac{1}{8}(\bar{D} D)^{2}\left(\Omega_{+} \Omega_{-}\right)-\frac{1}{2} \bar{D} D\left[V\left(\Omega_{+}\right)+V\left(\Omega_{-}\right)\right],
$$

where, $\Omega_{+}$and $\Omega_{-}, \bar{D}$ and $D$ are completely symmetrical.

For the quantum unified action from the interaction of quantum gravity and quantum repulsion, new variables are introduced: the square root $\sigma_{i}{ }^{a}$ of the three-dimensional metric and the potential $A_{a}{ }^{i}$ of the curvature self duality. The dynamic equation of the quantum unified action is as follows:

$$
\begin{array}{r}
\dot{\tilde{\sigma}}^{a}=\sqrt{2}^{ \pm} D_{b}\left(i \underline{T} \tilde{\sigma}_{\sigma}^{[b-a]}+T_{\sigma}^{[b-a]}\right), \\
{ }^{ \pm} A_{a}=\frac{1}{\sqrt{2}}\left(\left[i \underline{T} \tilde{\sigma}^{b},{ }^{ \pm} F_{a b}\right]-T^{b \pm} F_{a b}\right) .
\end{array}
$$

On the one hand, we consider the symmetry of supergravity ${ }^{[122]-[124]}$ : graviton vs gravitino; on the other hand, we introduce and consider the symmetry of superrepulsion: repulsion and repulstino.

Gravitons and gravitinos form supersymmetric relationships, which leads to the formation of high dimensional hypergravitons.

Repulsons and repulstinos form supersymmetric relationships, which leads to the formation of high dimensional hyperrepulsons.

Furthermore, between the high-dimensional supergraviton and the high-dimensional superrepulson, we introduce the high-dimensional supersymmetric arche-conjugate pulsaton (super coupling).

On the one hand, the quantum aspect of dark matter is attributed to quantum gravitons, on the other hand, the quantum aspect of dark energy is attributed to quantum repulsons.

Furthermore, we introduce and analyze the quantum conjugate (super coupling) pulsaton between the quantum aspect of dark matter as a quantum graviton and the quantum aspect of dark energy as a quantum repulson.

The application of ultimate supersymmetry to unified action theory leads to the introducing of 
ultimate unified action. The ultimate supersymmetry includes gravitational supersymmetry and repulsive supersymmetry, in which the gravitational supersymmetry is a theory constructed by adding a graviton ( whose $\operatorname{spin} s=2$ ) as a boson to a gravitino ( whose spin $s=3 / 2$ ) as a Fermi partner ${ }^{[125]-[126] \text {, }}$ while the repulsive supersymmetry is a theory constructed by adding a repulson to a repulstino.

( 3 ) Arche-Conjugation

We will not only consider the dynamic dark energy and the interacting dark energy, but also introduce and consider the arche-conjugate dark energy.

We will not nonly consider the dynamic dark matter and the interacting dark matter, but also introduce and consider the arche-conjugated dark matter.

Let $V$ be the volume of space, and the action of scalar disturbance can be given by the following formula:

$$
S=\frac{V}{2} \int_{k<k_{0}} d \eta d^{3} k \approx z^{2}(\eta)\left(\zeta_{-k}^{\prime} \zeta_{k}^{\prime}-c_{s}^{2} k^{2} \zeta_{-k} \zeta_{k}\right),
$$

where the apostrophe represents the differential of the new time coordinate $\eta, \zeta_{k}$ is the curvature disturbance and $z \equiv a \sqrt{2 \varepsilon_{1}} / c_{s}$ is Mukhanov variable.

The equation of motion satisfied by the variable $\zeta_{k}$ is:

$$
u_{k}^{\prime \prime}+\left(c_{s}^{2} k^{2}-\frac{z^{\prime \prime}}{z}\right) u_{k}=0 \text {. }
$$

This equation can be obtained from the variation of action ( 234 ), where the modulus function is defined as $u_{k}=z \zeta_{k}$. In the case of slow-rolling approximation, equation ( 235 ) is simplified. After the first order terms of slow roll parameter and $\lambda$ are retained, the following results are obtained:

$$
v \approx \frac{3}{2}+\varepsilon_{1}+\frac{\varepsilon_{2}}{2}-s-\frac{2}{3} \lambda
$$

The motion equation of tensor perturbation is almost the same as that of scalar perturbation, but the definition of Mukhanov variable becomes $z=a$. The specific disturbance equation is as follows:

$$
v_{k}^{\prime \prime}+\left(k^{2}-\frac{a^{\prime \prime}}{a}\right) v_{k}=0,
$$

where the modular function is defined as $v_{k} \equiv a h k / 2$. It should be noted that $h_{k}$ here represents two physical freedom degrees of tensor perturbation, namely $h_{+}$and $h_{x}$. To define conjugate momentum and its quantization:

$$
\begin{gathered}
\pi_{k}=\mu_{k}^{\prime}-\frac{a^{\prime}}{a} \mu_{k}, \\
\mu_{k}(\eta)=\frac{1}{\sqrt{2 k}}\left(a_{k}(\eta)+a_{-k}^{\dagger}(\eta)\right), \\
\pi_{k}(\eta)=-i \sqrt{\frac{k}{2}}\left(a_{k}(\eta)-a_{k}^{\dagger}(\eta)\right) .
\end{gathered}
$$

Under the special conformal time $\eta_{0}$, by the Bogoliubov transformation, we have: 


$$
\begin{aligned}
& a_{k}(\eta)=u_{k}(\eta) a_{k}\left(\eta_{\mathbf{0}}\right)+v_{k}(\eta) a_{-k}^{\dagger}\left(\eta_{\mathbf{0}}\right), \\
& a_{k}(\eta)=u_{k}(\eta) a_{-k}^{\dagger}\left(\eta_{\mathbf{0}}\right)+v_{k}(\eta) a_{k}\left(\eta_{\mathbf{0}}\right) .
\end{aligned}
$$

So in $\mu_{k}, \pi_{k}$, there should be

$$
\begin{gathered}
\mu_{k}(\eta)=f_{k}(\eta) a_{k}\left(\eta_{\mathbf{0}}\right)+f_{k}^{*}(\eta) a_{-k}^{\dagger}\left(\eta_{\mathbf{0}}\right), \\
\pi_{k}(\eta)=-i\left(g_{k}(\eta) a_{k}\left(\eta_{\mathbf{0}}\right)\right)+g_{k}^{*}(\eta) a_{-k}^{\dagger}\left(\eta_{\mathbf{0}}\right) .
\end{gathered}
$$

In fact, the arche-conjugation introduced in this series of work is the requirement of super dynamic equilibrium, that is to say, the arche-conjugation is the fundamental requirement for the natural formation of the universe between high-dimensional supergravity and high-dimensional superrepulsion, between quantum gravity and quantum repulsion, as well as between classical gravity and classical repulsion.

For the high-dimensional super unified action between the high-dimensional supergravity and the high-dimensional superrepulsion, the quantum unified action between the quantum gravity and the quantum repulsion, and the classical unified action between the classical gravity and the classical repulsion, the disequilibrium is the actual form (normal state), the equilibrium is the ideal form (abnormal state), therefore, in the normal state, the degree of deviation of the equilibrium is non-zero; in the abnormal state, the degree of deviation of the equilibrium is zero. Imbalance always drives the super forces to form deviation, while equilibrium always requires the super forces to shorten the deviation. Here are three situations:

( a ) $V_{\text {sugra }}(\Phi)-V_{\text {surepl }}(\Phi)=0$

the effective potential of supergravity and that of superrepulsion reach equilibrium;

( b ) $V_{\text {sugra }}(\Phi)-V_{\text {surepl }}(\Phi) \leq 0$,

the effective potential of supergravity is less than or equal to that of superrepulsion;

(c) $V_{\text {sugra }}(\Phi)-V_{\text {surepl }}(\Phi)>0$,

the effective potential of super gravity is larger than that of super repulsion.

In the super-inflationary universe model of ultra-synergy physics established later in this series, a mechanism (the quantum statistical physics process of nonlinear non-equilibrium) that combines the generation of baryon asymmetry with the super-inflation and reheating process of the universe will be studied.

( 4 ) Resonance at the Same Frequency

Now, a basic inference is given: at the level close to quantum gravity and quantum repulsion, all the gravitons and gravitinos, as well as all the repulsons and repulstinos, are in the co-frequency resonance state.

In our opinion, a series of major knotty problems, such as the singularity, inflation and accelerated expansion of the universe, involve super gravity, dark energy and dark matter. Super gravity, dark energy and dark matter are three closely related problems. In essence, dark energy and dark matter are 
problems of quantum gravity and quantum repulsion.

The coupling of dark matter and dark energy is attributed to the interaction of quantum gravity and quantum repulsion.

The decoupling of dark matter and dark energy is attributed to the separation of quantum gravity and quantum repulsion.

( 5 ) quantum hedge-unification

Under the big unified framework of quantum gravitational field and quantum repulsive field, this series of work comprehensively expands the general relativity and loop quantum ring gravitation theory, establishes the quantum repulsive field equation corresponding to the quantum gravitational field equation, and then establishes the quantum hedge-unified field equation describing the interaction of quantum gravitational field and quantum repulsive field; On this basis, the quantum repulsive universe equation corresponding to the quantum gravitational universe equation is established, thus a set of new equations describing the expansive universe model under the condition of fully considering the hedge effect of quantum gravitational field and quantum repulsion field, which can be called the expansive universe equation of quantum hedge-unified field theory, is established.

For the classical flat FRW universe (Friedmann-Robertson-Walker), the Hamiltonian constraint of its gravitational action can be simply expressed as

$$
H_{c l, \text { grav }}=-\frac{3}{8 \pi G \gamma^{2}} \sqrt{p_{\text {grav }}} c^{2}+H_{M}
$$

where, the momentum of gravity $p_{\text {grav }}=1 / a^{2}, c=\gamma \dot{a}, \gamma \approx 0.2375, a$ is the scale factor, and $H_{M}$ is the Hamiltonian of the gravitational mass. Here, define the energy density: $\pi \equiv \frac{E}{V}=\frac{H_{M}}{a^{3}}$.

When the loop quantum gravity effect cannot be ignored, the Hamiltonian constraint of the original gravity is modified as follows:

$$
H_{Q, \text { grav }}=-\frac{3}{8 \pi G \gamma^{2} \bar{\mu}^{2}} \sqrt{p} \sin ^{2}(\bar{\mu} c)+H_{M}
$$

where $\bar{\mu}$ is the length of the loop that makes up the smallest facet. When $\bar{\mu} \rightarrow 0$, there is $\sin ^{2}(\bar{\mu} c) / \bar{\mu}^{2} \rightarrow 1$, then $H_{Q, \text { grav }} \rightarrow H_{c l, \text { grav }}$ will return to the classical limit. Thus the modified Friedmann equation related to gravity is obtained:

$$
H_{\text {grav }}^{2}=\frac{8 \pi G}{3} \rho\left(1-\frac{\rho}{\rho_{c}}\right)
$$

where the critical density is

$$
\rho_{c}=\frac{\sqrt{3}}{16 \pi^{2} \gamma^{3}} \rho_{p}, \quad \rho_{p}=G^{-2} \text { is the first density of Planck. }
$$

The appearance of the critical density $\rho_{c}$ is completely a quantum gravitational effect. 
For the classical flat FRW universe (Friedmann-Robertson-Walker), the Hamiltonian constraint of repulsion can be simply expressed as

$$
H_{c l, r e p l}=-\frac{3 G}{8 \pi} \gamma^{2} \sqrt{p_{r e p l}} c^{2}+H_{E}
$$

where, the momentum of repulsion $p_{\text {repl }}=a^{2}, c=\gamma \dot{a}, \gamma \approx 0.2375, a$ is the scale factor, and $H_{E}$ is the Hamiltonian of the repulsive energy. Here, define the energy density: $\pi \equiv \frac{E}{V}=\frac{H_{E}}{a^{3}}$.

When the loop quantum repulsion effect cannot be ignored, the Hamiltonian constraint of the original repulsion is modified as follows:

$$
H_{Q, r e p l}=-\frac{3 G \bar{\mu}^{2}}{8 \pi} \gamma^{2} \sqrt{p_{\text {repl }}} \sin ^{2}\left(\frac{1}{\bar{\mu}} c\right)+H_{E}
$$

where $\bar{\mu}$ is the length of the loop that makes up the smallest facet. When $\bar{\mu} \rightarrow 0$, there is $\sin ^{2}\left(\frac{1}{\bar{\mu}} c\right) \bar{\mu}^{2} \rightarrow 1$, then $H_{Q, \text { repl }} \rightarrow H_{c l, r e p l}$ will return to the classical limit. Thus the modified non Friedmann equation related to repulsion is obtained:

$$
H_{r e p l}^{2}=\frac{8 \pi}{3 G} \sigma\left(1-\frac{\sigma}{\sigma_{c}}\right)
$$

where the critical density is

$$
\sigma_{c}=\frac{\sqrt{3} \gamma^{3}}{16 \pi^{2}} \sigma_{p}, \quad \sigma_{p}=G^{2} \text { is the first density of Planck. }
$$

The appearance of the critical density $\sigma_{c}$ is completely a quantum repulsive effect.

On the basis of the above discussion, for the classical flat FRW universe (Friedmann-Robertson -Walker), the Hamiltonian constraint of the grand unification of gravity and repulsion can be simply expressed as

$$
H_{c l, \text { repl }}-H_{c l, \text { grav }}=-\frac{3 G}{8 \pi} \gamma^{2} \sqrt{p_{\text {repl }}} c^{2}+H_{E}+\frac{3}{8 \pi G \gamma^{2}} \sqrt{p_{\text {grav }}} c^{2}-H_{M}
$$

When the loop quantum repulsion effect and the loop quantum gravitational effect cannot be ignored, the Hamiltonian constraint of the original hedge between the repulsion and the gravitation is modified as follows:

$$
H_{Q, \text { repl }}-H_{Q, \text { grav }}=-\frac{3 G \bar{\mu}^{2}}{8 \pi} \gamma^{2} \sqrt{p_{\text {repl }}} \sin ^{2}\left(\frac{1}{\bar{\mu}} c\right)+H_{E}+\frac{3}{8 \pi G \gamma^{2} \bar{\mu}^{2}} \sqrt{p} \sin ^{2}(\bar{\mu} c)-H_{M}
$$

where $\bar{\mu}$ is the length of the loop that makes up the smallest facet. 


$$
\text { When } \frac{1}{\bar{\mu}}-\bar{\mu} \rightarrow 0 \text {, there is } \bar{\mu}^{2} \sin ^{2}\left(\frac{1}{\bar{\mu}} c\right)-\frac{1}{\bar{\mu}^{2}} \sin ^{2}(\bar{\mu} c) \rightarrow 1 \text {, }
$$

then $H_{Q, \text { repl }}-H_{Q, \text { grav }} \rightarrow H_{c l, h e d g e}$ will return to the classical limit. Thus the modified quantum hedgeunified expansive universe equation related to the quantum repulsion and the quantum gravitation is obtained:

$$
H_{r e p l}^{2}-H_{\text {grav }}^{2}=\frac{8 \pi}{3 G} \sigma\left(1-\frac{\sigma}{\sigma_{c}}\right)-\frac{8 \pi G}{3} \rho\left(1-\frac{\rho}{\rho_{c}}\right)
$$

where the critical density is

$$
\begin{gathered}
\sigma_{c}-\rho_{c}=\frac{\sqrt{3} \gamma^{3}}{16 \pi^{2}} \sigma_{p}-\frac{\sqrt{3}}{16 \pi^{2} \gamma^{3}} \rho_{p}, \\
\sigma_{p}-\rho_{p}=G-\frac{1}{G^{2}} \text { is the first density of Planck. }
\end{gathered}
$$

The appearance of the critical density $\sigma_{c}$ of the hedge matter ( mass and energy ) is completely a quantum hedge effect.

Therefore, the semi-classical quantum hedg-unified cosmology shows us the evolution of the universe from contraction to rebound and then to expansion.

( 6 ) holographic quantum unification

At the level of quantum gravity and quantum repulsion, a holographic quantum unifying interaction mechanism that can be promoted to the basic principle has been explored and proposed earlier in this series. This mechanism discussed in this paper is derived from the holographic principle and holographic dark energy model, and the holographic principle is closely related to the entropy of black hole ${ }^{[127]-[131]}$.

When the loop quantum gravity effect cannot be ignored, the original Hamiltonian constraint is modified as follows:

$$
H_{L Q C}=-\frac{3}{8 \pi G \gamma^{2} \bar{\mu}^{2}} \sqrt{p} \sin ^{2}(\bar{\mu} c)+H_{M}
$$

where $\bar{\mu}$ is the length of the circle that makes up the smallest facet. When $\bar{\mu} \rightarrow 0$, there is $\sin ^{2}(\bar{\mu} c) / \bar{\mu}^{2} \rightarrow 1, H_{L Q C} \rightarrow H_{c l}$ will return to the classical limit.

The modified Friedmann equation and energy conservation equation are given

$$
\begin{gathered}
H^{2}=\frac{8 \pi G}{3}\left(\rho_{\text {grav }}+\rho_{\text {repl }}\right)\left(1-\frac{\rho_{\text {grav }}+\rho_{\text {repl }}}{\rho_{c}}\right), \\
\dot{\rho}_{\text {tot }}+3 H\left(1+w_{t o t}\right) \rho_{\text {tot }}=0
\end{gathered}
$$

where, $H=\frac{\dot{a}}{a}$ is the Hubble parameter, $\dot{a}$ represents the derivative of $a$ with respect to time $t . \rho_{\text {grav }}$ 
and $\rho_{\text {repl }}$ are the energy density dominated by quantum gravity and the energy density dominated by quantum repulsion (dark energy density related to cosmological constant), respectively. Their critical density is

$$
\rho_{c}=\rho_{\text {grav }, c}+\rho_{\text {repl }, c}=\frac{\sqrt{3}}{16 \pi^{2} \gamma^{3}} \rho_{p}, \quad \rho_{p}=G^{-2} \text { is Planck density. }
$$

The appearance of the critical density $\rho_{c}$ is a quantum effect. It makes the total energy density $\rho_{\text {tot }}$ $=\rho_{\text {grav }}+\rho_{\text {repl }}$ of the universe no longer diverge in any case. Therefore, the curvature of space-time is no longer diverging. In addition, $w_{t o t}=\frac{p_{t o t}}{\rho_{t o t}}$ is the state parameter of the total energy density.

Considering the interaction between matter and dark energy, $\rho_{\text {grav }}$ and $\rho_{\text {repl }}$ no longer meet the energy conservation equation alone, but should meet the balance equation:

$$
\dot{\rho}_{\text {grav }}+3 H \rho_{\text {grav }}=-Q, \quad \dot{\rho}_{\text {repl }}+3 H\left(1+w_{\text {repl }}\right) \rho_{\text {repl }}=Q,
$$

where, $Q$ is the unified term of interaction between quantum gravity and quantum repulsion.

Using the dimensionless density parameter $\Omega_{\text {grav }}=\rho_{\text {grav }} / \rho_{\text {grav }, c}, \Omega_{\text {repl }}=\rho_{\text {repl }} / \rho_{\text {repl }, c}$, the Hubble scale $H$ is chosen as the infrared truncation scale, and the energy truncation of the five dimensional quantum universe is done. The density of the holographic dark energy is $\rho_{\text {repl }}=\rho_{A}=B H$, $\rho_{A}$ is the dark energy density related to the cosmological constant $A$, and $B$ is the constant. we can get:

$$
\frac{\dot{H}}{H^{2}}=-\frac{\Omega_{r e p l}^{\prime}}{\Omega_{\text {repl }}}=-\frac{\Omega_{\Lambda}^{\prime}}{\Omega_{\Lambda}} .
$$

In order to investigate the early skyrocketing and the present accelerating expansion of the universe, it is necessary to calculate the deceleration factor $q$. By equation ( 258 ), we can get:

$$
\begin{gathered}
(1+z) \frac{2-\Omega_{\Lambda}}{\Omega_{\Lambda}} \frac{d \Omega_{\Lambda}}{d z} \frac{1}{\Omega_{\Lambda}}+3\left(1-\Omega_{\Lambda}\right)+3 c=0, \\
w^{e f f}=-1-(1+z) \frac{1}{3} \frac{d \Omega_{\Lambda}}{d z} \frac{1}{\Omega_{\Lambda}}, \\
q=-1-(1+z) \frac{d \Omega_{\Lambda}}{d z} \frac{1}{\Omega_{\Lambda}},
\end{gathered}
$$

It can be seen from equation ( 259 ) that in the future, the value of the effective state parameter will approach to -1 , but it will not exceed -1 . This shows that there will be no future of big tears in the universe. Even in the infinite future, $z \rightarrow-1$, the first term of formula ( 259 ) tends to zero.

As long as it is recognized that the dark energy component is still increasing at present $(z=0)$, the value of $\Omega_{A}$ will always be less than $1+c$. Therefore, from equation ( 259 ) and equation ( 260 ), we can see that the value of $w^{\text {eff }}$ is always greater than and tends to -1 .

Therefore, we propose a basic inference that can be promoted to the hyperholographic synergy 
principle of high dimensional universe.

The interaction between supergravity and superrepulsion in the complex evolution of the high-dimensional universe forms a dual relationship with the interaction between quantum gravity and quantum repulsion in the early inflation of the 4-dimensional universe.

The interaction between quantum gravity and quantum repulsion in the early inflation of the 4-dimensional universe form a dual relationship with the interaction between quantum-classical gravity and quantum-classical repulsion in the late accelerating expansion of the 4-dimensional universe.

Further, we propose a basic inference that can be promoted to the principle of hyperholography of high-dimensional universe (or multiuniverse): in a parallel universe formed by mutual coupling and holographic correspondence between the $P$ universe dominated by negative pressure (high-dimensional superrepulsion and quantum repulsion of accumulating positive energy, mainly composed of dark energy) and the $N$ universe dominated by positive pressure (high-dimensional supergravity and quantum gravity of accumulating negative energy, mainly composed of dark matter), there is a correspondence between the small-scale physics (ultraviolet cutoff) of the $P$ universe and the large-scale physics (infrared cutoff) of the $N$ universe, while there is a correspondence between the large-scale physics (ultraviolet cutoff) of the $P$ universe and the small-scale physics (infrared cutoff) of the $N$ universe.

( 7 ) ultra-synergy

The foregoing in this series has discussed the paradigm dynamical behavior of our early quantum universe $U$ in the Multi-verse world system (large heat reservoir) $W$, which corresponds to the Heisenberg image. Hamiltonian of total system $W \oplus U$

$$
H_{T O L}=H_{W}+H_{U}+H_{U W}=H_{0}+H_{U W}
$$

here $H_{W}$ is the Hamiltonian of $W$ itself, $H_{U}$ is the Hamiltonian of $U$ itself.

The relevant Liouville operator can also be written as

$$
L_{T O L}=L_{W}+L_{U}+L_{U W}=L_{0}+L_{U W}
$$

All the mechanical quantities of the total system $W \oplus U$ constitute the $C^{*}$ algebra $u^{T O L}$.

We can take the cosmic paradigm vector as an extension of the cosmic paradigm and describe it from $S$ sides. Consider here: $u_{1}$. energy density, $u_{2}$. accumulation intensity, $u_{3}$. spatiotemporal dimension (high dimension, no upper limit, including fractal dimension), $u_{4}$. curvature, $u_{5}$. expansion rate (or contraction rate), $u_{6}$. inhomogeneity, $u_{7}$. concentration, $u_{8}$. fluctuation range, etc.

If the temperature of the multi-verse world system as a heat reservoir is $\beta^{-1}$, the inner product can be introduced into $u^{T O L}$ :

$$
\left(K_{1}, K_{2}\right)=\operatorname{Tr} K_{1}^{+} K_{2} \rho_{T O L, 0}, \quad K_{1}, K_{2} \in u^{T O L}
$$

here $\rho_{T O L, 0}=e^{-\beta H_{T O L}} / \operatorname{Tr} e^{-\beta H_{T O L}}$. By formula ( 264 ), we introduce the distance

$$
\left\|K_{1}-K_{2}\right\|=\sqrt{\left(K_{1}-K_{2}, K_{1}-K_{2}\right)}
$$

We can make $u^{T O L}$ complete and get the Hilbert space $\mathscr{H}_{\beta}{ }^{T O L}$. The total Liouville operator $L$ is Hermitian in $\mathscr{H}_{\beta}{ }^{T O L}$. What we really care about is the paradigm vectors of the early quantum universe 
$U$, which constitute a subspace $\mathscr{H}_{\beta}{ }^{U}$ of $\mathscr{H}_{\beta}^{T O L}$. If the vector $\underline{e}=\left\{e_{i}\right\}_{i}$ is a set of bases in $\mathscr{H}_{\beta}{ }^{T O L}$, then the motion of the $U$ system is completely given by the temporal behavior of $\underline{e}(t)$.

The generalized Langevin equation can be regarded as the mode dynamical equation of the open quantum gravi-system $S$ in Heisenberg's image, and the density matrix motion equation of the quantum gravi-system $S$ can also be discussed from Schrodinger's image (Liouville equation).

The density matrix $\rho_{\text {TOL }}$ of ultra-synergistic system $B \oplus S$ satisfies the liouville Equation:

$$
\frac{d}{d t} \rho_{T O L}=-i\left(L_{0}+L_{B S}\right) \rho_{T O L}
$$

here $L_{0}=L_{B}+L_{S}$. Of course, we only focus on the mean value of the mode vector of the quantum gravi-system $S$ :

$$
<e>=\operatorname{Tr} e \rho_{T O L}=\operatorname{Tr}_{S} e\left(\operatorname{Tr}_{B} \rho_{T O L}\right)=\operatorname{Tr}_{S} e \rho_{S}
$$

Therefore, we hope to get the equation of motion satisfied by $\rho$ s. Setting projection operator $P$ :

$$
P \rho_{T O L}=\rho_{B_{0}} \operatorname{Tr}_{B} \rho_{T O L}
$$

$\rho_{B 0}$ is a traceable operator of $B$ system: $\operatorname{Tr}_{B} \rho_{B_{0}}=1$, its selection depends on the specific problem.

Let $Q=1-P$, so we get

$$
\frac{d}{d t} \rho_{S}(t)=-i L_{u, S} \cdot \rho_{S}(t)-\int_{0}^{t} d \tau K(t-\tau) \cdot \rho_{S}(\tau)+F(t)
$$

here it uses $P L_{B}=0$ and $P L_{S}=L_{S} P$, and there is

$$
\begin{gathered}
L_{u, S}=\operatorname{Tr}_{B} L \rho_{B_{0}}=L_{S}+\operatorname{Tr}_{B} L_{B S} \rho_{B_{0}} \\
K(\tau)=\rho_{B 0}^{-1} P L Q e^{-i Q L Q \tau} Q L P=\operatorname{Tr}_{B} L_{B S} e^{-i Q L Q \tau} Q\left(L_{B}+L_{B S}\right) \rho_{B 0}
\end{gathered}
$$

If the change of $K(\tau)$ is much faster than that of $\rho^{\prime}{ }_{U}(t)$, the Markov approximation can be introduced :

$$
\int_{0}^{t} d \tau K(\tau) e^{-i L_{S} t} \cdot \rho_{S}^{\prime}(t-\tau) \approx \int_{0}^{\infty} d \tau K(\tau) \rho_{S}(t)=\theta \rho_{S}(t)
$$

We get Master equation :

$$
\frac{d}{d t} \rho_{S}(t)=-i L_{e, S} \cdot \rho_{S}(t)-\theta \cdot \rho_{S}(t)
$$

\section{Acknowledgements}

I would like to express my sincere gratitude to the domestic and foreign researchers, my colleagues and relatives. Without their enlightening instruction, impressive kindness and help, I could not have completed my work.

\section{References}

[1] Guth A H. The inflationary universe: A possible solution to the horizon and flatness problems [J]. Phys Rev D, 1981, 23( 347) : 347 - 356.

[2] Linde A D. A new inflationary universe scenario: A possible solution of the horizon, flatness, homogeneity, 
isotropy and primordial monopole problems [J]. Phys Lett B, 1982, 108( 6) : 389 - 393.

[3] Linde A D. Chaotic inflation [J]. Phys Lett B, 1983,129, 3 -4: 177 - 181.

[4] Kofman L, Linde A D. Problems with tachyon inflation [J]. J of High Energy Physics, 2002( 7) , 004, 1 - 11.

[5] Renata Kallosh, Andrei Linde, Evan McDonough, Marco Scalisi. dS Vacua and the Swampland, JHEP, Jan 7 , 2019. 10 pp. 134

[6] Renata Kallosh, Andrei Linde, Evan McDonough, Marco Scalisi. de Sitter Vacua with a Nilpotent Superfield, Aug 28, 2018. Fortsch. Phys. 6 pp.

[7] Andrei Linde, Dong-Gang Wang, Yvette Welling, Yusuke Yamada, Ana Achúcarro. Hypernatural inflation, Mar 27, 2018. JCAP, 25 pp.

[8] Yashar Akrami, Renata Kallosh, Andrei Linde, Valeri Vardanyan. Dark energy, $\alpha$-attractors, and large-scale structure surveys, Dec 27, 2017. JCAP, 56 pp.

[9] Ana Achúcarro, Renata Kallosh, Andrei Linde, Dong-Gang Wang, Yvette Welling. Universality of multi-field $\alpha$-attractors, Nov 26, 2017. JCAP, $26 \mathrm{pp}$.

[10] Fresse K, Frieman J A, Olinto A V., Natural inflation with pseudo-Nambu-Goldstone bosons [J] . Phys Rev Lett, 1990, 65: 3233 -3236.

[11] R. R. Caldwell, M. Kaminonkowshi, N. N. Winberg, Phys. Rev. Lett, 91, 2003, 071301

[12] A R Menda, R Iz-Picon C, Damou R T, Mukhanov F. K-inflation [J]. Phys Lett B, 1999, 458( 2-3): 209-218.

[13] Gar R Iga J, Mukhanov V F. Perturbations in K-inflation [J]. Phys Lett B 1999, 458( 2 - 3) : 219 - 225.

[14] Andrei Linde. On the problem of initial conditions for inflation, Oct 11, 2017. Found. Phys. 15 pp.

[15] Li M, Yoneya T. D, particle dynamics and the space-time uncertainty relation [J]. Phys Rev Lett, 1997, 78 (7) : 1219 - 1222 .

[16] Li H, Xia J Q, Zhang X. Global fitting analysis on cosmological models after BICEP2 data 0/OlJ. arXiv: 1404. 0238 [astro -ph. CQ].

[17] Wu F, Li Y, Lu Y, et al. Cosmological parameter fittings with the BICEP2 data Q/Ol] . arXiv • 1403. 6462 [astro - ph. CO].

[18] Sen A. Rolling tachyon [J]. Journal of High Energy Physics, 2002( 04) , 048, 1 - 17.

[19] Sen A. Tachyon matter [J]. Journal of High Energy Physics, 2002( 07) , 065, 1 - 17.

[20] Gibbons G W. Cosmological evolution of the rolling tachyon [J]. Phys Lett B, 2002, 537( 1 - 2) : 1 - 4.

[21] Alishahiha M, Silve R Stein E, Tong D. DBI in the sky [J]. Phys Rev D, 2004, 70( 12) : 123505.

[22] Kofman L, Linde A D. Problems with tachyon inflation [J]. Journal of High Energy Physics, 2002( 7) , 004, 1 -11 .

[23] Fai R Bair N M, Tytgat M H G. Inflation from a tachyon fluid [J]. Phys Lett B, 2002, 546( 1 - 2) : 1 - 7.

[24] Piao Y S, Cai R G, Zang X M, et al. Assisted tachyonic inflation [J]. Phys Rev D, 2002, 66( 12) : 121301.

[25] G. Hao, X. Z. Li, Phantom-like GCG and the constraints of its parameters via cosmological dynamics, Phys. Lett. B606, 2005, 7

[26] Shin'ichi Nojiri, Sergei D. Odintsov, Inhomogeneous Equation of State of the Universe: Phantom Era, Future Singularity and Crossing the Phantom Barrier, Phys. Rev. D 72: 023003, 2005

[27] Shin'ichi Nojiri, Sergei D. Odintsov, Unifying phantom inflation with late-time acceleration: scalar phantom-non-phantom transition model and generalized holographic dark energy, Gen. Rel. Grav. 38: 1285-1304, 2006

[28] J. G. Hao, X. Z. Li, Phantom-like GCG and the Constraints of Its Parameters via Cosmological Dynamics, Phys. Rev. D68, 2003, 043501

[29] Feng C J, Li X Z, Sa Ridakis E N. Preventing eternality in phantom inflation [J]. Phys Rev D, 2010, 82(2) : 
023526.

[30] Hguang Q G, Li M. CMB power spectrum from noncommutative space-time [J]. Journal of High Energy Physics, 2003 ( 6) , 014, 1 - 6.

[31] Tsujikawa S, Maa R Tens R, B R Andenbe R Ge R R., Noncommutative inflation and the CMB [J]. Phys Lett B, 2003, 574( 3 - 4) : 141 - 148.

[32] Huang Q G, Li M. Noncommutative inflation and the CMB multipoles [J]. Journal of Cosmology and Astroparticle Physics, 2003, 11, 001, 1 - 10.

[33] Huang Q G, Li M. Power spectra in spacetime noncommutative inflation [J]. Nucl Phys B, 2005, 713 ( 1 3 ) : 219- 234 .

[34] Cai Y F, Wang Y. Noncommutative eternal inflation possible [J]. Journal of Cosmology and Astroparticle Physics, 2007, 06: 022, 1 - 10.

[35] Hinshaw G., Larson D, Komatsu E, et al. (WMAP Collaboration). Nine-year Wilkinson Microwave Anisotropy Probe ( WMAP) observations: Cosmological parameter results [J]. Astrophys J Suppl 2013, 208( 2) : 19.

[36] Ade P A R, Aghanim N, A R Mitage-Caplan C, et al. (Planck Collaboration). Planck 2013 results. XXII. Constraints on inflation[J /OL]. arXiv: 1303. 5082[astro - ph. CO].

[37] Ade P A R, Aikin R W, Ba R Kats D, et al. (BICEP2 Collaboration). BICEP2 I: Detection of B-mode polarization at degree angular scales[J /OL]. arXiv: 1403. 3985[astro - ph. CO].

[38] T. Padmanabhan, Cosmological constant - the weight of the vacuum, Phys. Rept. 380, 2003, 235

[39] Yoneya T. String theory and space-time uncertainty principle [J]. Prog Theor Phys, 2000, 103(6): 1081-1125.

[40] B R Andenbe R Ge R R , HO P M. Noncommutative space-time, stringy space-time uncertainty principle, and density fluctuations [J]. Phys Rev D, 2002, 66( 2) : 023517.

[41] William E. East et al, Beginning inflation in an inhomogeneous universe, Journal of Cosmology and Astroparticle Physics, 09(2016) 010

[42] Sabharwal, S., Khanna, G.: Numerical solutions to lattice-refined models in loop quantum cosmology. Class. Quantum Grav. 25, 085009, [arXiv:0711.2086] (2008)Google Scholar

[43] Bojowald, M.: The semiclassical limit of loop quantum cosmology. Class. Quantum Grav. 18, L109-L116, [gr-qc/0105113] (2001)Google Scholar

[44] Bojowald, M.: Homogeneous loop quantum cosmology. Class. Quantum Grav. 20, 2595 - 2615, [gr-qc/0303073] (2003)Google Scholar

[45] Salvatore Capozziello, Rocco D’Agostino and Orlando Luongo, Extended gravity cosmography, Int Journal of Modern Physics D, Volume 28, Issue 10, July 2019.

[46] R. S. Moraes, P. K. Sahoo, Barkha Taori and Parbati Sahoo, Phantom energy-dominated universe as a transient stage in $f(R)$ cosmology, International Journal of Modern Physics D, Volume 28, Issue 10, July 2019.

[47] Olga Razina, Pyotr Tsyba, Bekdaulet Meirbekov and Ratbay Myrzakulov, Cosmological Einstein-Maxwell model with g-essence, International Journal of Modern Physics D, Volume 28, Issue 10, July 2019.

[48] G. Oliveira-Neto, L. G. Martins, G. A. Monerat and E. V. Corrêa Silva, Quantum cosmology of a HořavaLifshitz model coupled to radiation, International Journal of Modern Physics D, Volume 28, Issue 10, July 2019.

[49] Sujoy K. Modak, Cosmological particle creation beyond de Sitter, International Journal of Modern Physics D, Volume 28, Issue 9, July 2019.

[50] Esraa Elkhateeb, Dissipative unified dark fluid model, International Journal of Modern Physics D, Volume 28, Issue 9, July 2019.

[51] Donatello Dolce. Compact Time and Determinism for Bosons: Foundations. Foundations of Physics. 2010, 
41 (2): 178-203.

[52] S. L. Dubovsky, S. M. Sibiryakov, Spontaneous breaking of Lorentz invariance, black holes and perpetuum mobile of the 2nd kind, Phys.Lett. B638, 509-514, 2006.

[53] Alday, Luis; Gaiotto, Davide; Tachikawa, Yuji, Liouville correlation functions from four-dimensional gauge theories. Letters in Mathematical Physics 91 (2), 2010: 167-197.

[54] Renata Kallosh, Andrei Linde, Evan McDonough, Marco Scalisi. dS Vacua and the Swampland, JHEP, Jan 7 , 2019. 10 pp. 134

[55] Renata Kallosh, Andrei Linde, Yusuke Yamada. Planck 2018 and Brane Inflation Revisited, Nov 2, 2018. JHEP, 29 pp.

[56] Renata Kallosh, Andrei Linde, Evan McDonough, Marco Scalisi. 4D models of de Sitter uplift, Sep 24, 2018. Phys. Rev. 4 pp.

[57] Renata Kallosh, Andrei Linde, Evan McDonough, Marco Scalisi. dS Vacua and the Swampland, JHEP, Jan 7, 2019. 10 pp. 134

[58] Renata Kallosh, Andrei Linde, Yusuke Yamada. Planck 2018 and Brane Inflation Revisited, Nov 2, 2018. JHEP, 29 pp.

[59] Renata Kallosh, Andrei Linde, Evan McDonough, Marco Scalisi. 4D models of de Sitter uplift, Sep 24, 2018. Phys. Rev. 4 pp.

[60] Yashar Akrami, Renata Kallosh, Andrei Linde, Valeri Vardanyan. The Landscape, the Swampland and the Era of Precision Cosmology, Aug 28, 2018. Fortsch. Phys. 48 pp.

[61] Renata Kallosh, Andrei Linde, Evan McDonough, Marco Scalisi. de Sitter Vacua with a Nilpotent Superfield, Aug 28, 2018. Fortsch. Phys. 6 pp.

[62] Andrei Linde, Dong-Gang Wang, Yvette Welling, Yusuke Yamada, Ana Achúcarro. Hypernatural inflation, Mar 27, 2018. JCAP, 25 pp.

[63] Yashar Akrami, Renata Kallosh, Andrei Linde, Valeri Vardanyan. Dark energy, $\alpha$-attractors, and large-scale structure surveys, Dec 27, 2017. JCAP, 56 pp.

[64] Ana Achúcarro, Renata Kallosh, Andrei Linde, Dong-Gang Wang, Yvette Welling. Universality of multi-field $\alpha$-attractors, Nov 26, 2017. JCAP, $26 \mathrm{pp}$.

[65] Andrei Linde. On the problem of initial conditions for inflation, Oct 11, 2017. Found. Phys. 15 pp.

[66] Emilio Elizalde, Martiros Khurshudyan and Shin'ichi Nojiri, Cosmological singularities in interacting dark energy models with an $\omega(q)$ parametrization, International Journal of Modern Phy. D, Vol 28, 1, July 2019.

[67] Bob Osano and Timothy Oreta, Multi-fluid theory and cosmology: A convective variational approach to interacting dark-sector, International Journal of Modern Physics D, Vol 28, 6, July 2019.

[68] Abhineet Agarwal, R. Myrzakulov, S. K. J. Pacif and M. Shahalam, Cosmic acceleration from coupling of baryonic and dark matter components: Analysis and diagnostics, International Journal of Modern Physics D, Vol 28, 6, July 2019.

[69] Aharony, Ofer; Bergman, Oren; Jafferis, Daniel Louis; Maldacena, Juan, N = 6 superconformal Chern -Simons-matter theories, M2-branes and their gravity duals. Journal of High Energy Physics 2008 (10): 091.

[70] Bilson-Thompson, Sundance O.; Markopoulou, Fotini; Smolin, Lee. Quantum gravity and the standard model. Classical and Quantum Gravity. 2007, 24 (16): 3975-3994.

[71] Connes, A., Non commutative dierential geometry, I the Chern character in K-homology. Publ. Math. IHES, 62 (1986), 41-144.

[72] Penrose, Roger, The twistor programme, Reports on Mathematical Physics 12 (1): 65-76, 1977 
[73] Cheng C, Huang Q G. Constraints on the cosmological parameters from BICEP2, Planck and WMAP[J /OL]. arXiv: 1403.7173 [astro - ph. CO].

[74] Ade P A R, Aikin R W, Ba R Kats D, et al. (BICEP2 Collaboration). BICEP2 I: Detection of B-mode polarization at degree angular scales[J /OL]. arXiv: 1403. 3985[astro - ph. CO].

[75] N. Agafonova et al, Measurement of the cosmic ray muon flux seasonal variation with the OPERA detector, Journal of Cosmology and Astroparticle Physics, 10(2019)003

[76] A. Challinor et al, Exploring cosmic origins with CORE: Gravitational lensing of the CMB, Journal of Cosmology and Astroparticle Physics, 04(2018)018

[77] Albrecht A, Steinhardt P J. Cosmology for grand unified theories with radiatively induced symmetry breaking HI . Phys Rev Lett, 1982,48 : 1220 - 1223.

[78] Gong Y. The challenge for single field inflation with BICEP2 result |j/01]. arXiv:1403.5716-qd.

[79] Chung D J H, Shiu G, Trodden M. Running of the scalar spectral index from inflationary models [J]. Phys Rev D, 2003,68: 063501.

[80] Easther R, Peiris H. Implications of a running spectral index for slow roll inflation [J]. JCAP, 2006,0609 : $010,1 \sim 8$

[81] Feng B, Li M Z, Zhang R J, et al. An inflation model with large variations in spectral index, Phys Rev D, 2003,68:103511. [astro - ph/030247].

[82] Kobayashi T, Takahashi F. Running spectral index from inflation with modulations 00. JCAP, 2011, 1101:026, 1-24.

[83] Feng C J, Li X Z, Is non-minimal inflation eternal [J]. Nucl Phys B, 2010, 841(1-2) : 178-187

[84] Feng Chaojun, Li Xinzhou, Inflationary Universe of Kinetic Energy in Noncommutative Time and Space, Astrophysics Center of Shanghai Normal University, Journal of Shanghai Normal University, 4. 2014, 43(4): 359 -368.

[85] Wang, Bin; Gong, Yungui; Abdalla, Elcio, Thermodynamics of an accelerated expanding universe, Physical Review D, vol. 74, Issue 8, 10, 2006, id. 083520 (PhRvD Homepage)

[86] Izquierdo, Germán; Pavón, Diego, Dark energy and the generalized second law, Physics Letters B, Volume 633, Issue 4-5, p. 420-426. 02, 2006

[87] Edmund J. Copeland, M. Sami, Shinji Tsujikawa, Dynamics of dark energy, Int. J. Mod. Phys. D15: 1753-1936, 2006

[88] Liu D J, Li X Z. Non-commutative power-law inflation: Mode equation and spectra index [J]. Phys Lett B, 2004, $600(1-2): 1-6$.

[89] Liu D J, Li X Z. Cosmological perturbations and noncommutative tachyon inflation [J]. Phys Rev D, 2004, 70 ( 12) : 123504.

[90] Feng C J, Li X Z, Liu D J. Note on Power-Law Inflation in Noncommutative Space-Time [J/OL]. arXiv: 1404. 0168 [astro - ph. CO].

[91] Li X Z, Liu D J, Hao J G. On the tachyon inflation [J]. J. Shanghai Normal Univ. (Natural Sciences), 2004, 33(4): 29- 34.

[92] Melia, Fulvio. The Cosmic Horizon: Monthly Notices of the Royal Astronomical Society, 2007, 382 (4): $1917-1921$

[93] Ma Y Z, Wang Y., Reconstructing the local potential of inflation with BICEP2 data U/O1J. arXiv: 1403. 4585 [astro-ph. CO].

[94] Choudhury S, Mazumdar A. Reconstructing inflationary potential from BICEP2 and running of tensor modes 0/ OlJ . arXiv: 1403.5549 [iep - th]. 
[95] Nakayama K, Takahashi F, Yanagida T T. Polynomial chaotic inflation in supergravity [J]. Journal of Cosmology and Astroparticle Physics, 2013, 8: 038, 1 - 18.

[96] Kobayashi T, Seto O. Polynomial inflation models after BICEP2 \&/OI]. arXiv: 1403. 5055 [astro-ph. CQ].

[97] R. Brandenberger, P. Ho, Non-commutative space-time, stringy space-time uncertainty principle and density fluctuations, Phys. Rev. D, 66, 2002, 023517, arXiv: hep-th/0203119

[98] S. Alexander, R. Brandenberger, J. Magueijo, Non-commutative inflation, Phys. Rev. D, 67, 2003, 081301; arXiv:hep-th/0108190

[99] Dolce, D. Gauge Interaction as Periodicity Modulation, Annals of Physics, Volume 327, Issue 6, June 2012, pp. $1562-1592$

[100] Rodolfo Gambini and Jorge Pullin, A First Course in Loop Quantum Gravity, Oxford, 2011

[101] Aharony, Ofer; Silverstein, Eva. Supercritical stability, transitions, and ( pseudo ) tachyons. Physical Review D 75 (4), 2007.

[102] Sacha Kocsis, Boris Braverman, et al.: Observing the Average Trajectories of Single Photons in a Two-Slit Interferometer, Science, vol. 332 no. 6034 pp.\&nbsp:1170-1173, 3 June 2011

[103] Bernstein, Jeremy, Max Born and the Quantum Theory. Am. J. Phys. 73 (11), November 2005: 999-1008.

[104] A. O. Bolivar: Classical limit of bosons in phase space, Physica A: Statistical Mechanics and its Applications, vol. 315, no. 3-4, December 2002, pp. 601-615

[105] B. J. Hiley, R. E. Callaghan: Delayed-choice experiments and the Bohm approach, Physica Scripta, vol. 74, no. 3, pp. 336 ff., 2006

[106] B. J. Hiley, R. E. Callaghan: Clifford Algebras and the Dirac-Bohm Quantum Hamilton-Jacobi Equation, Foundations of Physics, January 2012, Volume 42, Issue 1, pp 192-208

[107] Li X Z, Hao J G, Liu D J. Can quintessence be the rolling tachyon [J]. Chin Phys Lett, 2002, 19( 11) : 1584 - 1586.

[108] Li X Z, Zhai X H. The tachyon inflationary models with exact mode functions [J]. Phys R ev D, 2003, 67( 6): 067501.

[109] Zhang H, Li X Z, Noh H. Brane tachyon dynamics [J]. Phys Lett B, 2010, 691( 1): 1 - 10.

[110] Piao Y S, Huang Q G, Zhang X M, et al. Nonminimally coupled tachyon and inflation [J]. Phys Lett B, 2003, 570 (1-2): 1 - 4 .

[111] Zhang, B.; et al. Discerning the physical origins of cosmological gamma-ray bursts based on multiple observational criteria: the cases of $\mathrm{z}=6.7 \mathrm{GRB} 080913, \mathrm{z}=8.2 \mathrm{GRB}$ 090423, and some short/hard GRBs. Astrophysical Journal. 2009, 703 (2): 1696-1724.

[112] Abdo, A. A.; et al. Fermi Observations of High-Energy Gamma-Ray Emission from GRB 080916C. Science. 2009, 323 (5922): 1688-93.

[113] Berry M. V., Balazs N. L., Evolution of semiclassical quantum states in phase space, J. Phys. A12, 625, 1989.

[114] Ignazio Licata: Emergence and computation at the edge of classical and quantum systems, in: Ignazio Licata, Ammar Sakaji (eds.): Physics of Emergence and Organization, World Scientific, 2008, pp. 1-26

[115] B. Hiley: Weak values: Approach through the Clifford and Moyal algebras, Journal of Physics: Conference Series vol. 361, issue 1, 012014, 2012

[116] Dennis, Glen; de Gosson, Maurice A.; Hiley, Basil J. (2014). Fermi's ansatz and Bohm's quantum potential. Physics Letters A 378 (32-33): 2363-2366.

[117] William Seager, Classical Levels, Russellian Monism and the Implicate Order. Foundations of Physics, 
April 2013, Volume 43, Issue 4, pp. 548-567.

[118] Feng Lei, research on some problems of dark matter and dark energy, Doctoral Dissertation of Nanjing University, 2012

[119] Ioannis Papadimitriou, Supersymmetry anomalies in new minimal supergravity, Journal of High Energy Physics, 2019-09-16, Springer journal

[120] Hiroyuki Abe, Shuntaro Aoki, Sosuke Imai, Interpolation of partial and full supersymmetry breakings in N $=2$ supergravity, Nuclear Physics, Section B, 2019, 9. 3

[121] J. A. de Azcárraga, D. Gútiez, J. M. Izquierdo, Extended D = 3 Bargmann supergravity from a Lie algebra expansion, Nuclear Physics, Section B, 2019, 9.3

[122] Xiande Fang, Da Tang, Ling Zheng, Experimental investigation of gravity and channel size effects on flow boiling heat transfer under hypergravity, Aerospace Science and Technology, 2019, 9. 24

[123] Paolo Di Vecchia, Andrés Luna, Stephen G. Naculich, A tale of two exponentiations in N = 8 supergravity, Physics Letters B, 2019, 9

[124] Tony Pinhero, Supratik Pal, Realising mutated hilltop inflation in supergravity, Physics Letters B, 2019. 9. 5

[125] Kazunori Nakayama, A note on gravitational particle production in supergravity, Phy Letters B, 2019, 8. 29

[126] Yermek Aldabergenov, Auttakit Chatrabhuti, Sergei V. Ketov, Generalized dilatonaxion models of inflation, de Sitter vacua and spontaneous SUSY breaking in supergravity, The European Physical Journal C, 2019, 8. 26

[127] G.'t Hooft, Dimensional Reduction in Quantum Gravity, THU-93/26, gr-qc/ 9310026

[128] L. Susskind, The World as A Hologram, Math. Phys., 1995, 36:6377-6396

[129] Yuting Wang, Lixin Xu, Current Observational Constraints to Holographic Dark Energy Model with New Infrared cut-off via Markov Chain Monte Carlo Method, Phys. Rev. D 81: 083523, 2010

[130] N. Granda, A. Oliveros, New infrared cut-off for the holographic scalar fields models of dark energy, Physics Letter B, Vol. 671, 2, 19, 2009

[131] Jia Tian, Jue Hou, Bin Chen, Holographic correlators on integrable superstrata, Nuclear Physics, Section B, 2019, 9.24 